BNL-95236-2011

Formal Report

\title{
Opportunities for Drell-Yan Physics at RHIC
}

May $11-13,2011$

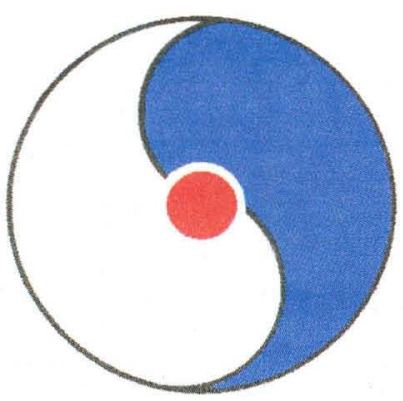

Organizers: Elke Aschenauer (BNL); Les Bland (BNL); Hank Crawford (U. of Berkeley); Yuji Goto (RIKEN/RBRC); Oleg Eyser (UC-Riverside); Zhongbo Kang (BNL/RBRC); Anslem Vossen (U. of Indiana)

\section{RIKEN BNL Research Center}

Building 510A, Brookhaven National Laboratory, Upton, NY 11973-5000, USA 


\section{DISCLAIMER}

This work was prepared as an account of work sponsored by an agency of the United States Government. Neither the United States Government nor any agency thereof, nor any of their employees, nor any of their contractors, subcontractors or their employees, makes any warranty, express or implied, or assumes any legal liability or responsibility for the accuracy, completeness, or any third party's use or the results of such use of any information, apparatus, product, or process disclosed, or represents that its use would not infringe privately owned rights. Reference herein to any specific commercial product, process, or service by trade name, trademark, manufacturer, or otherwise, does not necessarily constitute or imply its endorsement, recommendation, or favoring by the United States Government or any agency thereof or its contractors or subcontractors. The views and opinions of authors expressed herein do not necessarily state or reflect those of the United States Government or any agency thereof.

Notice: This manuscript has been authored by employees of Brookhaven Science Associates, LLC under Contract No. DE-AC02-98CH10886 with the U.S. Department of Energy. The publisher by accepting the manuscript for publication acknowledges that the United States Government retains a non-exclusive, paid-up, irrevocable, world-wide license to publish or reproduce the published form of this manuscript, or allow others to do so, for United States Government purposes. 


\section{Preface to the Series}

The RIKEN BNL Research Center (RBRC) was established in April 1997 at Brookhaven National Laboratory. It is funded by the "Rikagaku Kenkyusho" (RIKEN, The Institute of Physical and Chemical Research) of Japan. The Memorandum of Understanding between RIKEN and BNL, initiated in 1997, has been renewed in 2002 and again in 2007. The Center is dedicated to the study of strong interactions, including spin physics, lattice QCD, and RHIC physics through the nurturing of new generations of young physicists.

The RBRC has both a theory and experimental component. The RBRC Theory Group and the RBRC Experimental Group consists of a total of 25-30 researchers. Positions include the following: full time RBRC Fellow, half-time RHIC Physics Fellow, and full-time, post -doctoral Research Associate. The RHIC Physics Fellows hold joint appointments with RBRC and other institutions and have tenure track positions at their respective universities or BNL. To date, RBRC has 100 graduates of which 27 theorists and 14 experimenters have attained tenure positions at major institutions world wide.

Beginning in 2001 a new RIKEN Spin Program (RSP) category was implemented at RBRC. These appointments are joint positions of RBRC and RIKEN and include the following positions in theory and experiment: RSP Researchers, RSP Research Associates, and Young Researchers, who are mentored by senior RRC Scientists. A number of RIKEN Jr. Research Associates and Visiting Scientists also contribute to the physics program at the Center.

RBRC has an active workshop program on strong interaction physics with each workshop focused on a specific physics problem. In most cases all the talks are made available on the RBRC website. In addition, highlights to each speaker's presentation are collected to form proceedings which can therefore be made available within a short time after the workshop. To date there are one hundred and two proceedings volumes available.

A 10 teraflops RBRC QCDOC computer funded by RIKEN, Japan, was unveiled at a dedication ceremony at BNL on May 26, 2005. This supercomputer was designed and built by individuals from Columbia University, IBM, BNL, RBRC, and the University of Edinburgh, with the U.S.D.O.E. Office of Science providing infrastructure support at BNL. Physics results were reported at the RBRC QCDOC Symposium following the dedication. QCDSP, a 0.6 teraflops parallel processor, dedicated to lattice QCD, was begun at the Center on February 19, 1998, was completed on August 28, 1998, and was decommissioned in 2006. It was awarded the Gordon Bell Prize for price performance in 1998. The next generation computer in this sequence, QCDCQ (400 Teraflops), will become operational in the summer of 2011.

N. P. Samios, Director

February 2011 


\section{CONTENTS}

Preface to the Series

Organizers: Elke Aschenauer, Les Bland, Hank Crawford, Yuji Goto, Oleg Eyser, Zhongbo Kang, Anselem Vossen

Introduction by the orgainzers...........................................

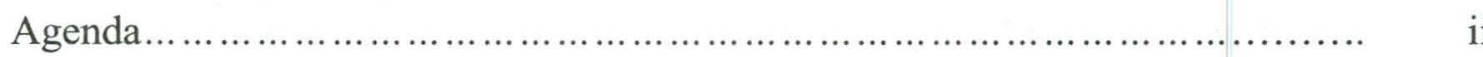

Introduction: Collinear and TMD factorization for Drell-Yan production

George Sterman (Stony Brook)...................................... 1

Low-x physics, saturation and forward Drell-Yan production Al Mueller (Columbia)................................................ 7

What semi-inclusive DIS has taught us about TMD's

Gunar Schnell ( $U$ of the Basque Country and IKERBASQUE)............... 13

Critique of TMD Phenomenology

Mauro Anselmino (U of Torino)...................................... 19

Sivers effect: from SIDIS to pp

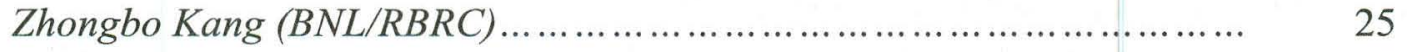

Gluon densities and di-hadron correlations

Bowen Xiao (Penn State U) ............................................... 31

From DIS to Drell-Yan Production through the Color Glass

Jamal Jalilian-Marian (Baruch College)............................. 37

What we can learn with Drell-Yan in proton(Deuteron) nucleus collisions

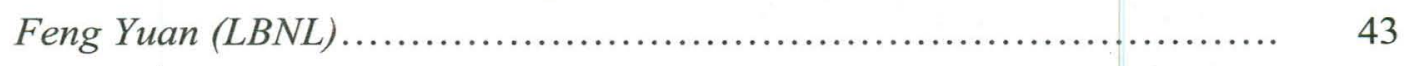

TMD Universality

Piet Mulders (UV U Amsterdam) .................................. 49

Guage Links and TMD-Factorization

Ted Rogers (VU U Amsterdam) ..................................... 55

Drell-Yan Production at FNAL-E906

Paul Reimer (ANL) .............................................. 61

Forthcoming Drell-Yan experiment at COMPASS

Oleg Denisov (INFN-Turin) .................................... 69

Other physics opportunities in future Drell-Yan experiments

Jen-Chieh Peng (UIUC) ......................................... $\quad 75$

Drell-Yan Production at STAR: Status and Plans

Ernst Sichtermann (LBNL)...................................... 81

Drell-Yan Production at PHENIX: Status and Plans

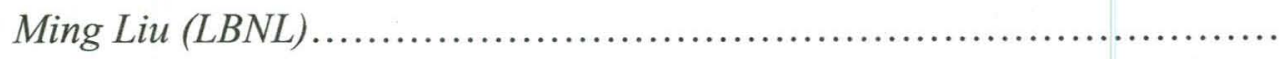


AnDY: Status and Plans

Les Bland.

RHIC: present status and future plans

Wolfram Fischer (BNL).

99

Theoretical perspectives on Drell-Yan production measurements

Jian-Wei Qiu (BNL).

105

TMD fracture functions in SIDIS and DY

Aram Kotzinian (Torino $U \& I N F N \& Y e r P h l$ ).

109

Discussions of Experimental Opportunities

What the Drell-Yan measurement can offer us at small-x?; Questions; What is the impact of DY on small-x

physics?

Generalized TMDs and Wigner distributions

Andreas Metz (Temple U)

Orbital angular momentum

Matthias Burkardt (NMSU).

Probing multi-gluon correlations in pp collisions

Yuji Koike (Niigata U).

Guage Links \& Process dependence in Hadronic Reactions

Leonard Gamberg (Penn State U)

Understanding forward particle production

Roman Pasechnik (Uppsala U)....

Drell-Yan at forward rapidities

Anna Stasto (Penn State U/RBRC)...

Sivers function in SIDIS, PP and DY

Alexei Prokudin (JLab)

Transverse physics with e+e-, SIDIS \& pp

Alessandro Bacchetta (U of Pavia \& INFN Pavia)

Photon Pair Production

Marc Schlegel (U of Tuebingen)

Many body QCD: from RHIC (\&LHC) to the EIC

Raju Venugopalan (BNL)

Transverse Single Spin Asymmetries for Drell-Yan production

John Collins (Penn State U)

List of participants

List of Past Proceedings

Contacts 


\section{Introduction to the workshop:}

Drell-Yan (DY) physics gives the unique opportunity to study the parton structure of nucleons in an experimentally and theoretically clean way. With the availability of polarized proton-proton collisions and asymmetric $d+A u$ collisions at the Relativistic Heavy lon Collider (RHIC), we have the basic (and unique in the world) tools to address several fundamental questions in QCD, including the expected gluon saturation at low partonic momenta and the universality of transverse momentum dependent parton distribution functions. A Drell-Yan program at RHIC is tied closely to the core physics questions of a possible future electron-ion collider, eRHIC. The more than 80 participants of this workshop focused on recent progress in these areas by both theory and experiment, trying to address imminent questions for the near and mid-term future. The talks and discussions were circling around and trying to interconnect between the following topics:

- Theoretical understanding of the transverse structure of the proton and the Sivers function in SIDIS and DY in particular.

- DY in polarized proton collisions: theoretical and experimental challenges.

- Current theoretical understanding of the low-x structure of nuclear matter (low-x PDFs (integrated and un-integrated) and Saturation).

- DY in $d+A u$ collision: What can we learn from asymmetric collisions?

- Future prospects: What can polarized He-3 collisions and $p+A u$ collisions teach us?

- Relation of DY@RHIC to physics at eRHIC. 


\section{Opportunities for Drell-Yan Physics at RHIC}

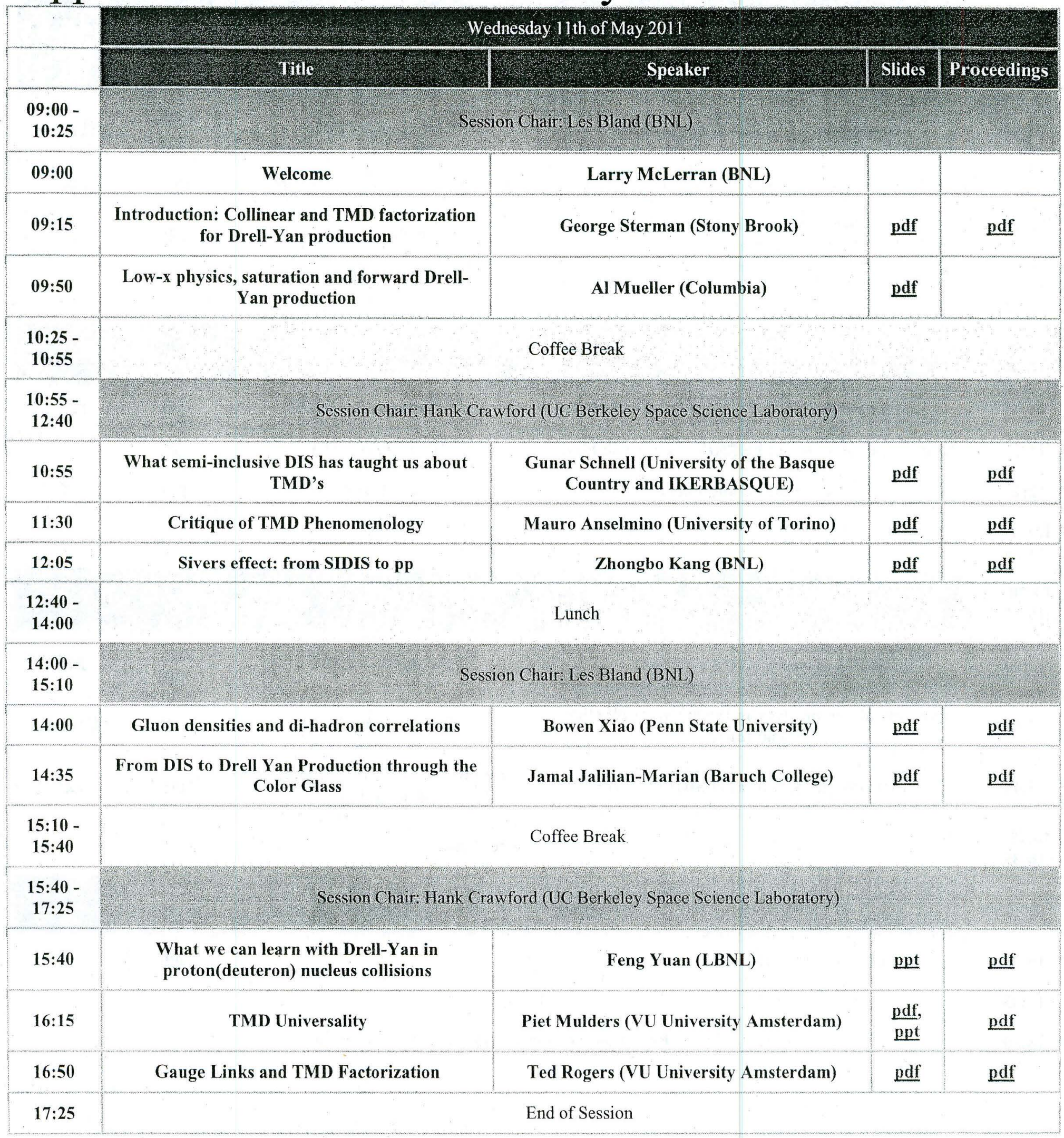




\begin{tabular}{|c|c|c|c|c|}
\hline & 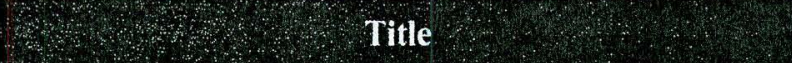 & Speaker & Slides & Proceedings \\
\hline \multicolumn{5}{|l|}{$\begin{array}{l}09: 00- \\
10: 45\end{array}$} \\
\hline 09:00 & Drell-Yan Production at FNAL-E906 & Paul Reimer (ANL) & $\underset{\text { pdf }}{\text { pdf }}$ & pdf pptx \\
\hline 09:35 & Forthcoming Drell-Yan experiment at COMPASS & Oleg Denisov (INFN - Turin) & ppt & pdf \\
\hline $10: 10$ & $\begin{array}{c}\text { Other physics opportunities in future Drell-Yan } \\
\text { experiments }\end{array}$ & Jen-Chieh Peng (UIUC) & pdf & pdf \\
\hline $\begin{array}{c}10: 45- \\
11: 15\end{array}$ & \multicolumn{4}{|c|}{ Coffee Break } \\
\hline $\begin{array}{c}11: 15- \\
13: 00\end{array}$ & \multicolumn{4}{|c|}{ Session Chair: Anselm Vossen (Indiana University) } \\
\hline $11: 15$ & Drell-Yan Production at STAR: Status and Plans & Ernst Sichtermann (LBNL) & pdf & pdf \\
\hline $11: 50$ & Drell-Yan Production at PHENIX: Status and Plans & Ming Liu (LANL) & pdf & pdf \\
\hline $12: 25$ & AnDY: Status and Plans & Les Bland & ppt & pdf \\
\hline $\begin{array}{c}13: 00- \\
14: 30\end{array}$ & \multicolumn{4}{|c|}{ Lunch } \\
\hline $\begin{array}{l}14: 30- \\
16: 15\end{array}$ & \multicolumn{4}{|c|}{ Session Chair: Oleg Eyser (UC Riverside) } \\
\hline $14: 30$ & RHIC: present status and plans & Wolfram Fischer (BNL) & $\frac{\text { pdf, }}{\text { pptx }}$ & pdf \\
\hline $15: 05$ & $\begin{array}{c}\text { Theoretical perspectives on Drell-Yan production } \\
\text { measurements }\end{array}$ & Jian-Wei Qiu (BNL) & pdf & pdf1, pdf2 \\
\hline $15: 40$ & TMD fracture functions in SIDIS and DY & $\begin{array}{c}\text { Aram Kotzinian (Torino Uni\&INFN } \\
\text { and YerPhI) }\end{array}$ & pdf & pdf \\
\hline $\begin{array}{c}16: 15- \\
16: 35\end{array}$ & \multicolumn{2}{|c|}{ Coffee Break } & & \\
\hline $\begin{array}{c}16: 35- \\
17: 45\end{array}$ & \multicolumn{2}{|c|}{ Session Chair. Anselin Vossen (Indiana University) } & & \\
\hline $16: 35$ & Discussion of Experimental Opportunities & & pdf & \\
\hline $17: 45$ & \multicolumn{2}{|c|}{ End of Session } & & \\
\hline $18: 30$ & \multicolumn{2}{|c|}{ Reception and Banquet at the Brookhaven Center } & & \\
\hline
\end{tabular}




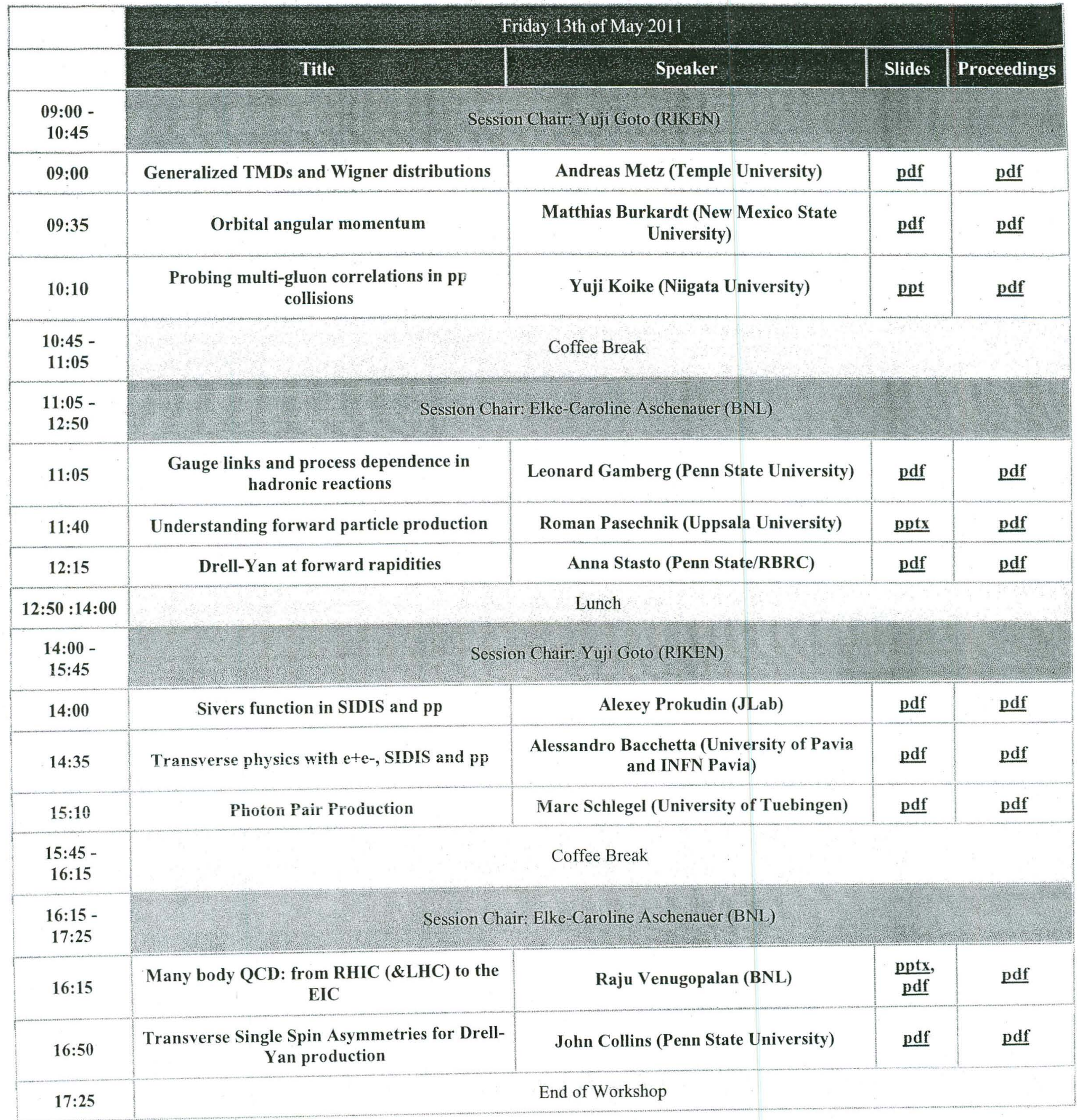




\title{
Introduction: Collinear and TMD Factorization for Drell-Yan Production
}

\author{
George Sterman, Stony Brook
}

This talk describes some general considerations to help set the stage for the workshop. Most

_ of what is included applies to both spin averaged and spin-dependent cross sections. In summary: Factorization in quantum field theory is closely related to classical considerations. Differences between initial- and final-state gauge links are consistent with this factorization. There is a well-developed theory of factorization for Drell-Yan, including transverse momentum $\left(Q_{T}\right)$ dependence. The 'QCD-inclusive' nature of Drell-Yan production maintains the underlying factorization. Nonperturbative effects play an essential role at low $Q_{T}$ and should be thought of as an integral part of the formalism. The stage is set for a new phenomenology to explore the transverse-momentum dependent and spin-sensitive parton distributions. 


\section{Drell-Yan Production in the Parton Model}

- The original 'collinear factorization'

- In the parton model (1970).

Drell and Yan: look for the annihilation of quark pairs into virtual photons of mass $Q \ldots$ any electroweak boson in NN scattering.

$$
\begin{aligned}
& \frac{d \sigma_{N N \rightarrow \mu \bar{\mu}+X}\left(Q, p_{1}, p_{2}\right)}{d Q^{2} d \ldots} \sim \\
& \int d \xi_{1} d \xi_{2} \sum_{a=\mathrm{q} \overline{\mathrm{q}}} \frac{d \sigma_{\mathrm{a} \bar{a} \rightarrow \mu \bar{\mu}}^{\mathrm{EW}, \mathrm{Born}}\left(Q, \xi_{1} p_{1}, \xi_{2} p_{2}\right)}{d Q^{2} d \ldots} \\
& \times\left(\text { probability to find parton a }\left(\xi_{1}\right) \text { in } N\right) \\
& \times\left(\text { probability to find parton } \overline{\mathrm{a}}\left(\xi_{2}\right) \text { in } N\right)
\end{aligned}
$$

The probabilities are $\phi_{q / N}\left(\xi_{i}\right)$ 's from DIS 


\section{The Physical Basis of Factorization}

- 'Collinear factorization' for hadron-hadron scattering for a hard, inclusive process with momentum transfer $M$ to produce final state $F+X$ :

$$
\begin{aligned}
& d \sigma_{\mathrm{H}_{1} \mathrm{H}_{2}}\left(p_{1}, p_{2}, M\right)= \\
& \sum_{a, b} \int_{0}^{1} d \xi_{a} d \xi_{b} d \hat{\sigma}_{a b \rightarrow F+X}\left(\xi_{a} p_{1}, \xi_{b} p_{2}, M, \mu\right) \\
& \quad \times \phi_{a / H_{1}}\left(\xi_{a}, \mu\right) \phi_{b / H_{2}}\left(\xi_{b}, \mu\right)
\end{aligned}
$$

- Factorization proofs: justifying the "universality" of the parton distributions. 
$-A=c B t^{\prime}-x_{3}^{\prime}$

$$
\begin{array}{lcc}
\text { field } & \underline{x \text { frame }} & \underline{x^{\prime} \text { frame }} \\
\text { scalar } & \frac{q}{|\vec{x}|} & \frac{q}{\left(x_{T}^{2}+\gamma^{2} \Delta^{2}\right)^{1 / 2}} \sim \frac{1}{\gamma} \\
\text { gauge (0) } & \boldsymbol{A}^{0}(\boldsymbol{x})=\frac{q}{|\vec{x}|} & \boldsymbol{A}^{\prime 0}\left(\boldsymbol{x}^{\prime}\right)=\frac{-q \gamma}{\left(x_{T}^{2}+\gamma^{2} \Delta^{2}\right)^{1 / 2}} \sim \gamma^{0} \\
\text { field strength } & E_{3}(\boldsymbol{x})=\frac{q}{|\vec{x}|^{2}} & E_{3}^{\prime}\left(x^{\prime}\right)=\frac{-q \gamma \Delta}{\left(x_{T}^{2}+\gamma^{2} \Delta^{2}\right)^{3 / 2}} \sim \frac{1}{\gamma^{2}}
\end{array}
$$

- The "gluon field" $A^{\prime \mu}$ is enhanced, yet is a total derivative:

$$
A^{\prime \mu}=q \frac{\partial}{\partial x_{\mu}^{\prime}} \ln \left(\Delta\left(t^{\prime}, x_{3}^{\prime}\right)\right)+\mathcal{O}(1-\beta) \sim A^{\prime-}
$$

- The "large" part of $A^{\prime \mu}$ can be removed by a gauge transformation! 


\section{TMD Factorization for Drell-Yan Production}

- $Q_{T}$ factorized cross sections: the motivation

- Low $Q_{T}$ Drell-Yan \& Higgs at leading $\log (\mathrm{LL})\left(\alpha_{s}{ }^{n} \ln ^{2 n-1} Q_{T}\right)$

$$
\frac{d \sigma(Q)}{d Q_{T}} \sim \frac{d}{d Q_{T}} \exp \left[-\frac{\alpha_{s}}{\pi} C_{F} \ln ^{2}\left(\frac{Q}{Q_{T}}\right)\right]
$$

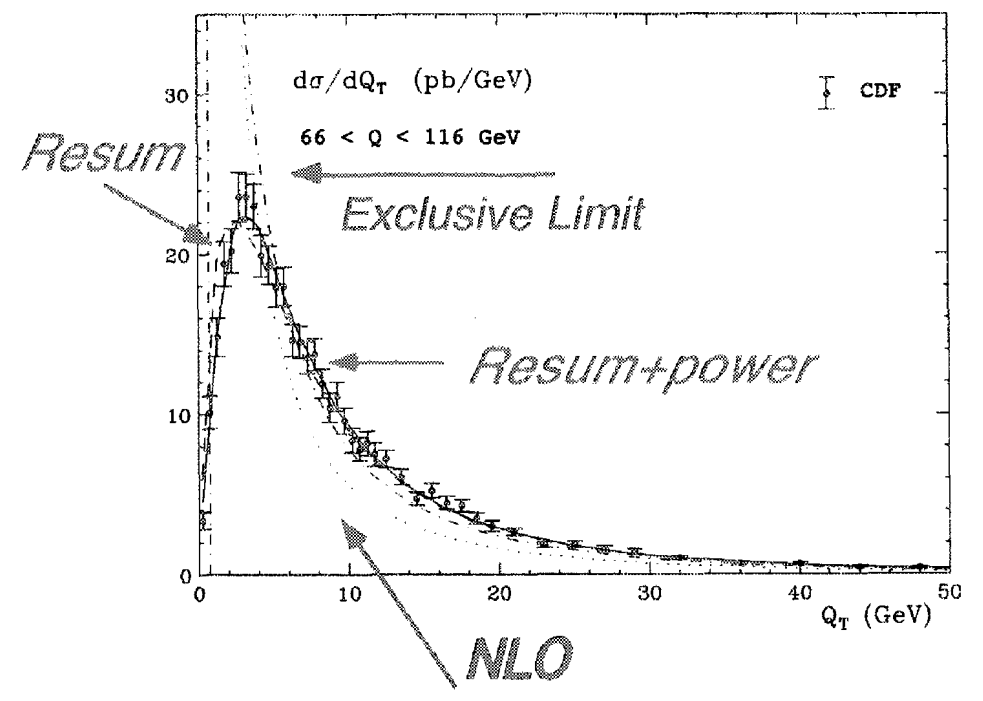


- Window to nonperturbative distributions:

$$
\begin{aligned}
\mathbb{E}^{\mathrm{soft}} & =\frac{1}{2 \pi} \int_{0}^{\mu_{T}^{2}} \frac{d^{2} k_{T}}{k_{T}^{2}} \mathbb{A}_{q}\left(\alpha_{s}\left(k_{T}\right)\right) \ln \left(\frac{Q^{2}}{k_{T}^{2}}\right)\left(e^{i \mathrm{~b} \cdot \mathrm{k}_{T}}-1\right) \\
& \sim-\int_{0}^{\mu_{T}^{2}} \frac{d k_{T}^{2}}{k_{T}^{2}}\left(\mathrm{~b} \cdot \mathrm{k}_{T}\right)^{2} \mathbb{A}_{q}\left(\alpha_{s}\left(k_{T}\right)\right) \ln \left(\frac{Q^{2}}{k_{T}^{2}}\right)+\cdots \\
& \sim-b^{2} \int d k_{T}^{2} \mathbb{A}_{q}\left(\alpha_{s}\left(k_{T}\right)\right) \ln \left(\frac{Q^{2}}{k_{T}^{2}}\right)
\end{aligned}
$$

$\theta\left(k_{T}-1 / b\right) \Rightarrow\left(e^{i \mathrm{~b} \cdot \mathrm{k}_{T}}-1\right) ;$ in fact, correct to all orders,

Note the expansion is for $b$ "small enough" only. 
Low-x physics, saturation and. Forward Drell-yan production

A.H. Mueller

Columbia university

The idea of saturation; the dipole picture Cresting dipoles

Physical processes in Forward deuteron (proton )-nucleus scattering

With Fominguez, S, Murder, B, Xian 
The Idea of Saturation; The Dipole Picture A. Dipole scattering is the Merman terugopalan
mode?

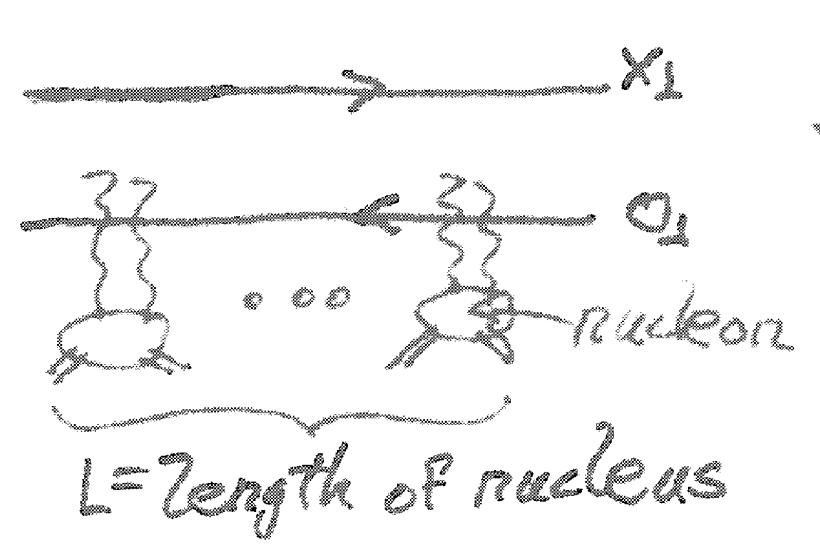

$$
\begin{aligned}
S(x, L) & =e^{-G_{\text {dip }}^{\infty} \rho / 2} \\
S^{2}(x, L) & =e^{-L / \lambda} \\
\lambda & =\frac{1}{\rho \sigma_{\text {dip }}}
\end{aligned}
$$

One also writes

$$
S\left(x_{1}, 4\right)=e^{-x_{1}^{2} \theta_{0}^{2} / 4} \text { when } x_{1}>\% \text { string seat. }
$$

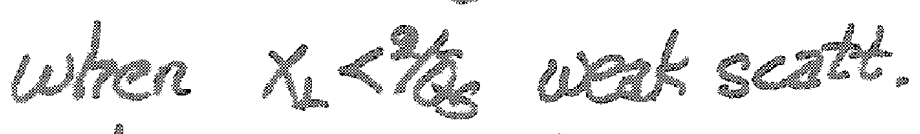

$\bar{Q}_{s}^{2}=$ quark saturation momentum $=\frac{C_{s}}{N_{c}} Q_{s}^{2}$

$$
Q_{s}^{2}=\frac{4 \pi^{2} \alpha N_{C}}{N_{\Delta}^{2}-1} \rho L \times G_{N}\left(x_{3}^{\left.1 / x_{1}^{2}\right)}\right.
$$

At $x=10^{-2}$ and $b_{2}=0 \quad a_{s}^{2} \approx 1 \mathrm{GeV}$ For large nucleus

The number of gluons per unit area or the nucleus is, when $x_{2} \leq \%$,

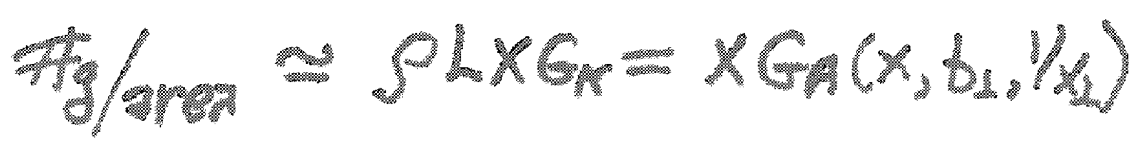

8 
The eccupation as

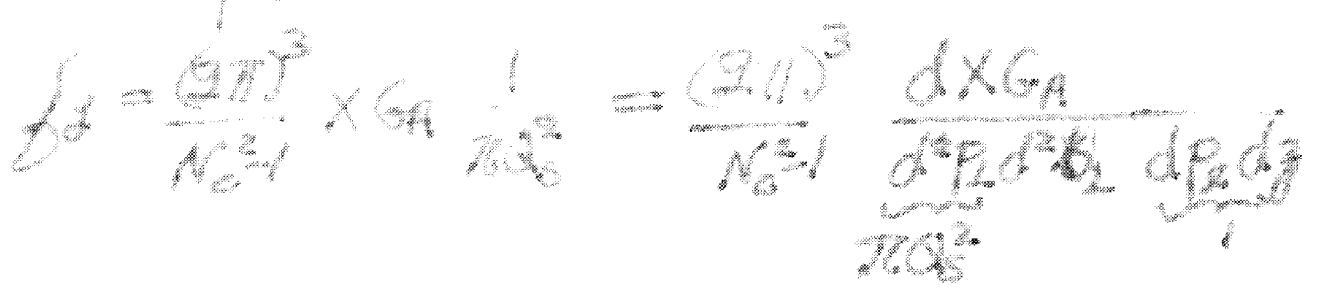

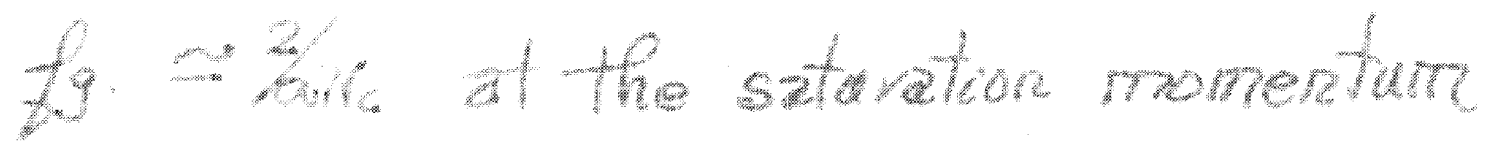

Hdingevolution

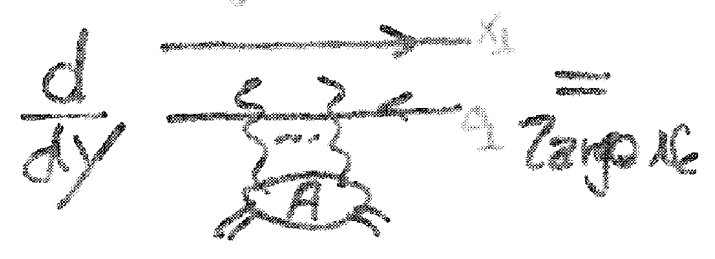

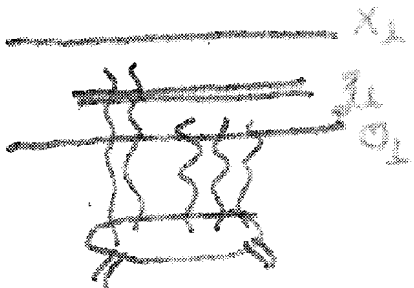

$$
\begin{aligned}
& \frac{d T\left(x_{1}, y\right)}{d y}=\frac{\alpha N_{c}}{2 \pi^{2}} \int d^{2} z_{1} \frac{x_{2}^{2}}{z_{1} x_{1} x_{1}} \cdot\left[T\left(x_{1}-z_{1}, y\right)+T\left(z_{1}, y\right)-T\left(x_{1}, y\right)\right.-\underbrace{\left.T\left(x_{1}-z_{1}, z_{1}, y\right)\right]}] \\
& T\left(x_{1}-z_{1}, y\right) \cdot T\left(\xi_{1}, y\right)
\end{aligned}
$$

15 Babtsky-kucheqor equation.

Tactorizes For Targe nucle: wher Ynot two Zange. In genera? $\mathrm{f}^{\mathrm{g}}$ Factorizes For lavge oceupancy on an event by event basis.

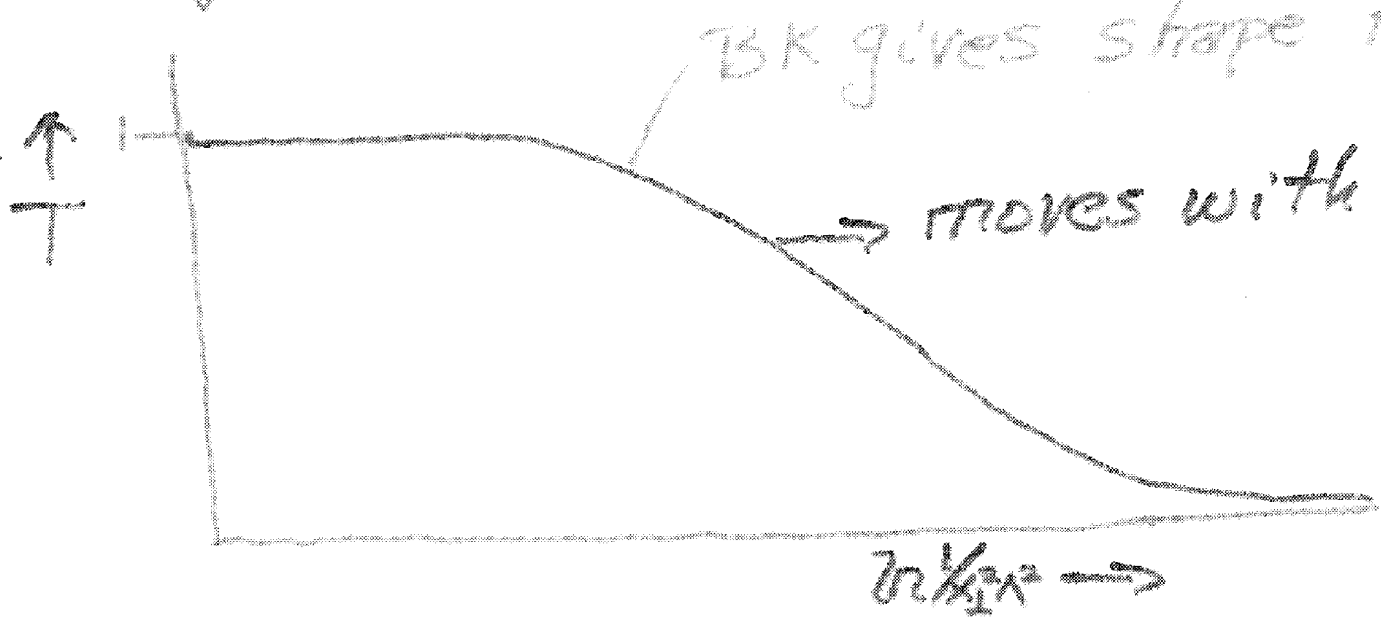


Creating Dipoles

Dipoles of a controllable size occur in $F_{L}\left(x, Q^{2}\right)$ and in ionia, but ave hand to build otherwise. However, to sone level?, they also occur in "easily" accessed? processes.

A. Pubradering in MV

Send guat through nucleus and measure the transverse momentum it peeks up

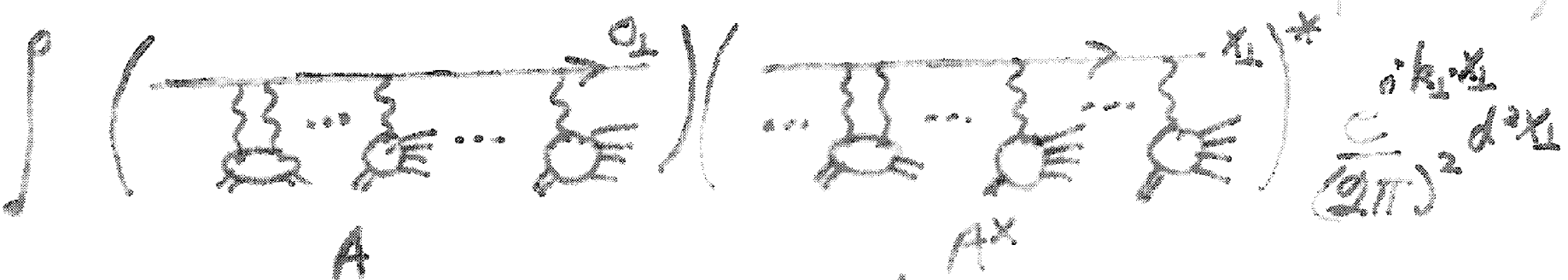

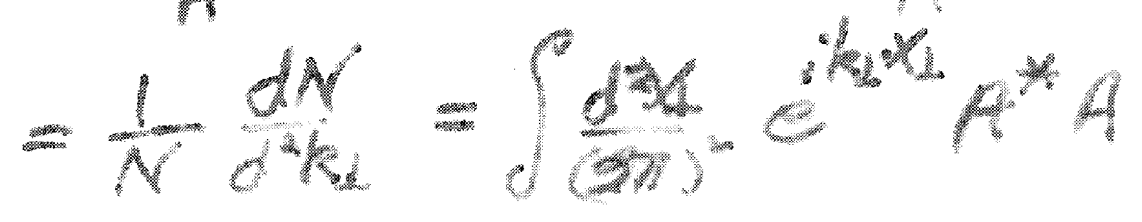

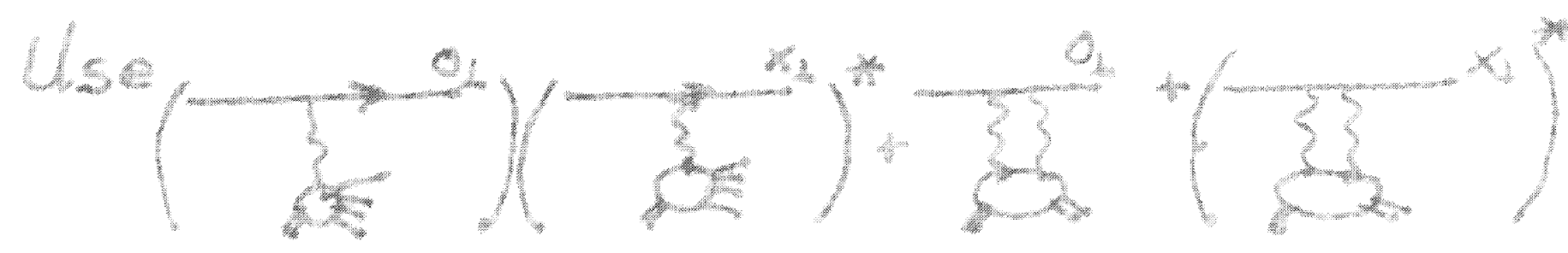

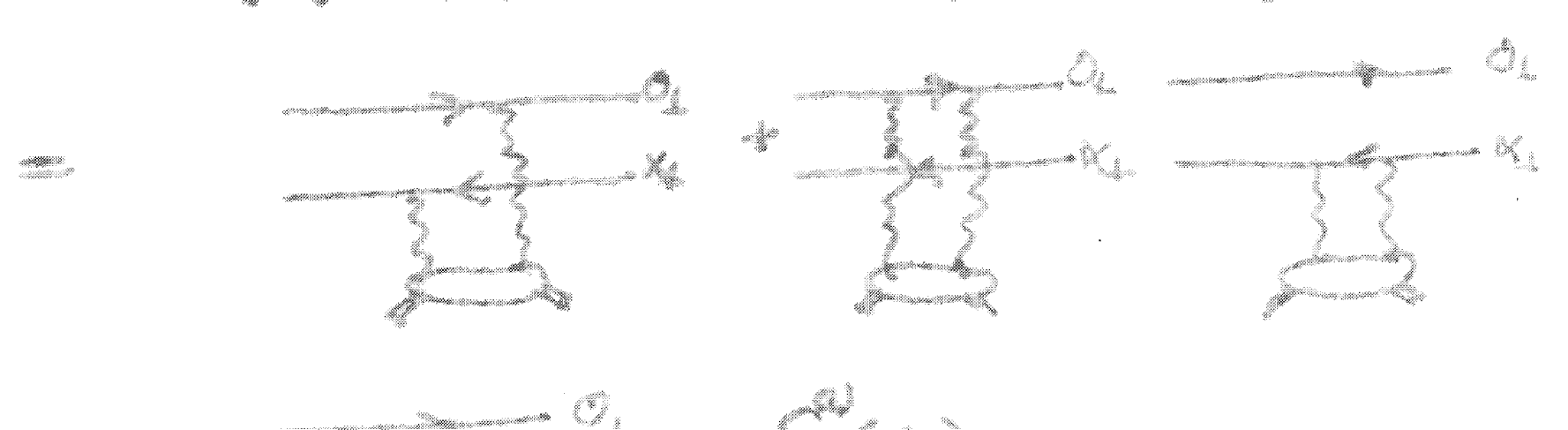

$$
=\frac{3}{3} x^{3}-S\left(x_{2}\right)
$$


Put in multiple scattering by Gabber

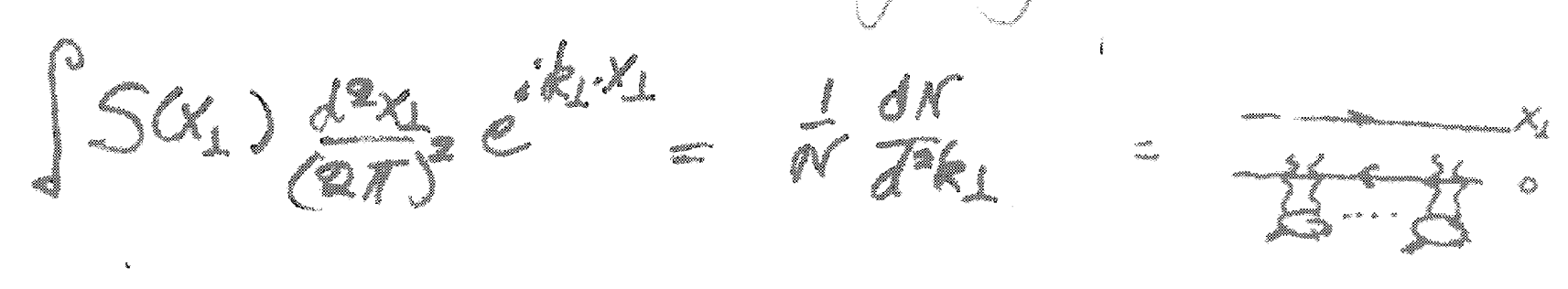

$k_{1}$-broadening jiver in terms of dipole scattering

E. Bring in expiation In general

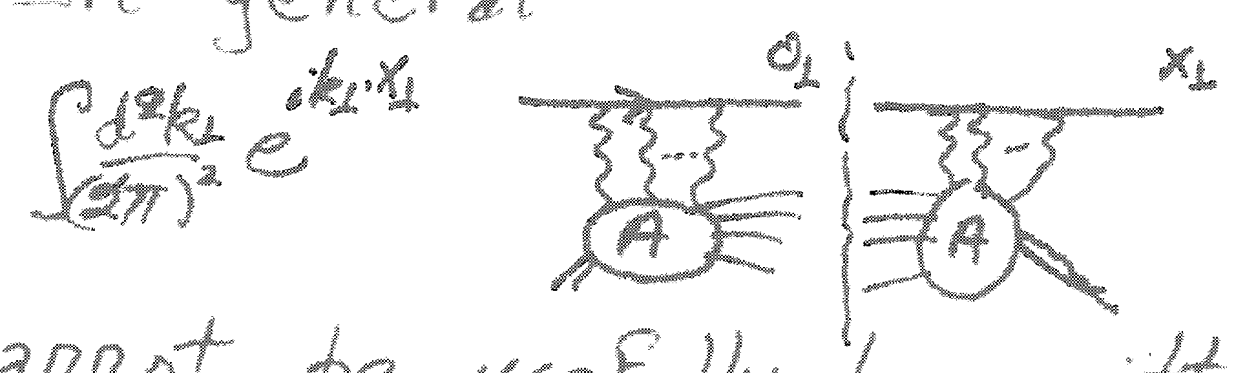

cannot be usefully be written in terms of a time-ordered product. Need two time formalism (or subtle discontonaity)

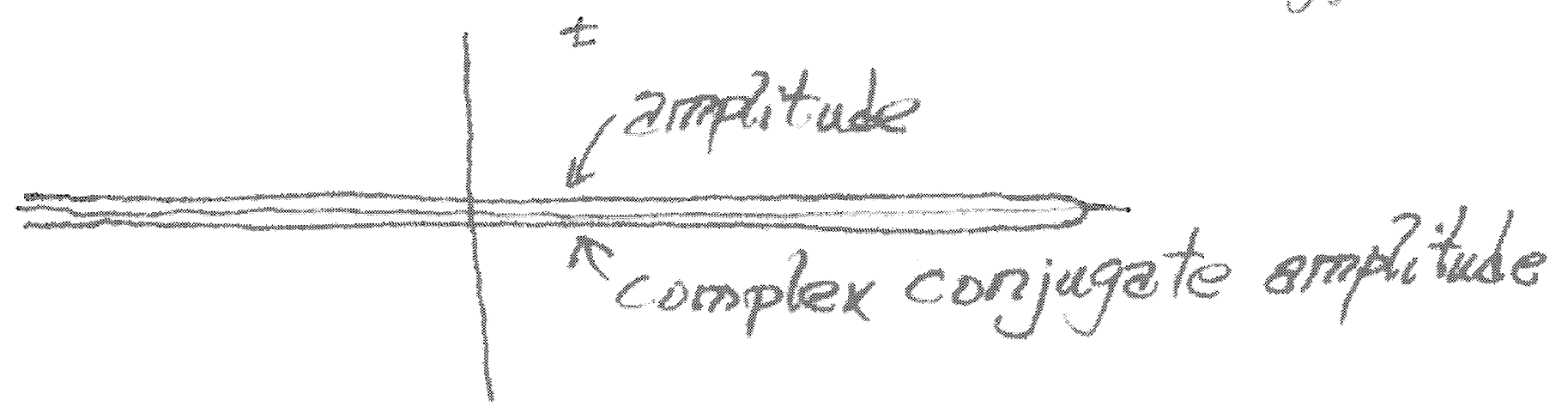

11 
However, at leading log level on can identify
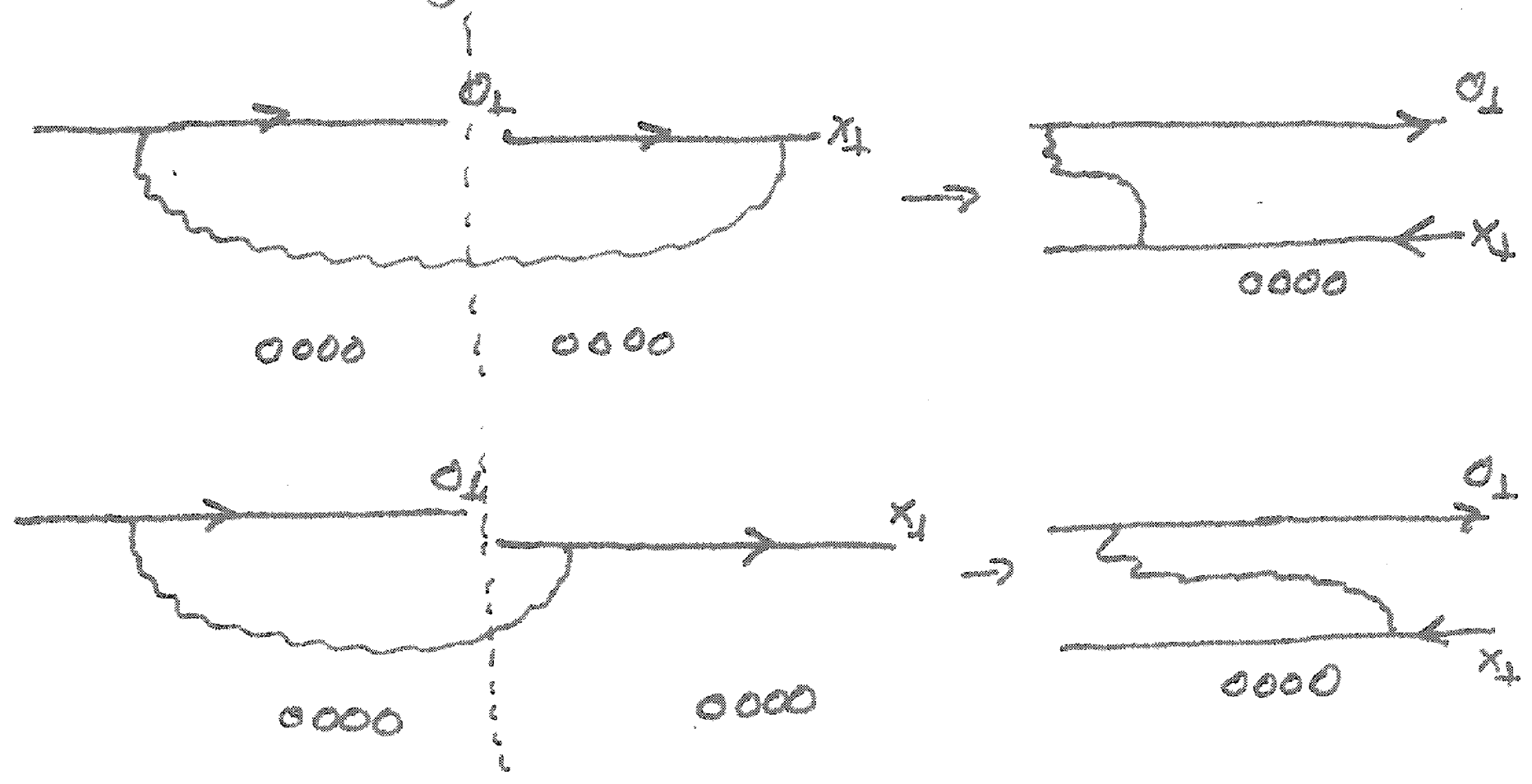

1-1 correspondence between dipole graphs and the corrections in the original process. OK for (O NCY) terms leading logs). Appears to break down at next to leading level.

should be ok to use dipole picture and BK equation to study P-broadening. But, higher order corrections still an open question.

12 


\title{
What semi-inclusive DIS has taught us about TMDs
}

\author{
G. SCHNELL \\ Department of Theoretical Physics, University of the Basque Country UPV/EHU, \\ 48080 Bilbao, Spain and \\ IKERBASQUE, Basque Foundation for Science, 48011 Bilbao, Spain
}

One of the cornerstones of modern hadronic physics is the recognition of the important role of partonic transverse momentum and spin-orbit correlations in the description of the nucleon structure. In semi-inclusive deep-inelastic scattering (DIS) these effects lead to sizable modulations in the azimuthal distribution of hadrons about the virtualphoton direction, which are parameterized in terms of several semi-inclusive structure functions. At leading order in an expansion in $M_{N} / Q$, the structure functions are described by transverse-momentum-dependent parton distribution and fragmentation functions (TMDs).

Evidence for TMDs in semi-inclusive DIS were found in the seminal measurement by the HERMES collaboration of pion production off longitudinally polarized protons. All interpretations of these data were hampered by the variety of TMD contributions to this measurement. Two major branches were followed: the interpretation in terms of the Collins fragmentation function and the interpretation as a signal for the naive- $T$-odd Sivers function. It took a few more years to shed additional light on the true origin of the observed single-spin asymmetries: measurements of similar asymmetries but on transversely polarized protons made it clear that both the Collins function and the Sivers function are non-zero; but the observed asymmetries off longitudinally polarized protons are caused by twist- 3 effects.

In the meantime, a wealth of data on TMDs have emerged. Recently the HERMES collaboration completed its analysis of the Sivers modulation using the entire available data set with transversely polarized protons. A manifest signal of non-zero Sivers functions for valence quarks was found, with strong indications that the Sivers distributions for up and down quarks are opposite in sign. This observation is supported by vanishing Sivers modulations measured by the CompAss collaboration. A surprisingly large signal for positive kaons was found by both collaborations, larger than the one for pions, implying a non-trivial role of sea quarks and/or of the underlying transverse-momentum dependences of the distributions and fragmentation functions.

Besides the Sivers modulation, the HERMES and COMPASS collaborations have data on the Collins effect with tantalizing large signals for $\pi^{-}$. In combination with the results for $\pi^{+}$, which are of opposite sign and smaller in size, it is conjectured that the disfavored Collins fragmentation, i.e., up quarks into $\pi^{-}$, is opposite in sign to and as large as the favored Collins fragmentation, i.e., up quarks into $\pi^{+}$. This can also be concluded when analyzing these data together with data from $e^{+} e^{-}$collision and from semi-inclusive DIS off transversely polarized deuterons.

Sivers, Collins, and transversity are not the only TMDs found to be non-zero. Up to date there are in addition signs for a non-vanishing distribution of longitudinally polarized quarks in transversely polarized nucleons from HERMES and JLAB, for the Boer-Mulders distribution, as well as for several subleading-twist distributions. 


\section{Spin-Momentum Structure of the Nucleon}

$\frac{1}{2} \operatorname{Tr}\left[\left(\gamma^{+}+\lambda \gamma^{+} \gamma_{5}\right) \Phi\right]=\frac{1}{2}\left[f_{1}+S^{i} \epsilon^{i j} k^{j} \frac{1}{m} f_{1 T}^{\perp}+\lambda \Lambda g_{1}+\lambda S^{i} k^{i} \frac{1}{m} g_{1 T}\right]$

$\frac{1}{2} \operatorname{Tr}\left[\left(\gamma^{+}-s^{j} i \sigma^{+j} \gamma_{5}\right) \Phi\right]=\frac{1}{2}\left[f_{1}+S^{i} \epsilon^{i j} k^{j} \frac{1}{m} f_{1 T}^{\perp}+s^{i} \epsilon^{i j} k^{j} \frac{1}{m} h_{1}^{\perp}+s^{i} S^{i} h_{1}\right.$

helicity quark pol.

$\left.+s^{i}\left(2 k^{i} k^{j}-k^{2} \delta^{i j}\right) S^{j} \frac{1}{2 m^{2}} h_{1 T}^{\perp}+\Lambda s^{i} k^{i} \frac{1}{m} h_{1 L}^{\perp}\right]$

I

\begin{tabular}{|c|c|c|c|}
\hline & $\mathrm{L}$ & $\mathrm{L}$ & $\mathrm{T}$ \\
\hline $\mathrm{U}$ & $f_{1}$ & & $h_{1}^{\perp}$ \\
\hline $\mathrm{L}$ & & $g_{1 L}$ & $h \frac{1}{1 c}$ \\
\hline $\mathrm{T}$ & $f_{1 T}^{\perp}$ & $g_{1 T}$ & $c_{1}, h_{1 T}$ \\
\hline
\end{tabular}

Sivers 'Twist - MDs

transversity
- fisnrtinne in hlack survive integration Boer-Mulders se momentum

- functions in green box are chirally odd

- functions in red are naive $T$-odd Mulders-Tangerman* *aka Pretzelosity 


\begin{tabular}{|c|c|c|c|}
\hline & $\mathrm{U}$ & $\mathrm{L}$ & $\mathrm{T}$ \\
\hline $\mathrm{U}$ & $f_{\mathrm{t}}$ & & $h_{1}^{\perp}$ \\
\hline $\mathrm{L}$ & & $g_{1 L}$ & $h_{1 L}$ \\
\hline $\mathrm{T}$ & $f_{1 T}^{\perp}$ & $g_{1}$ & $h_{1}, h_{T}$ \\
\hline
\end{tabular}

\section{Momentum density}

- plenty of data available

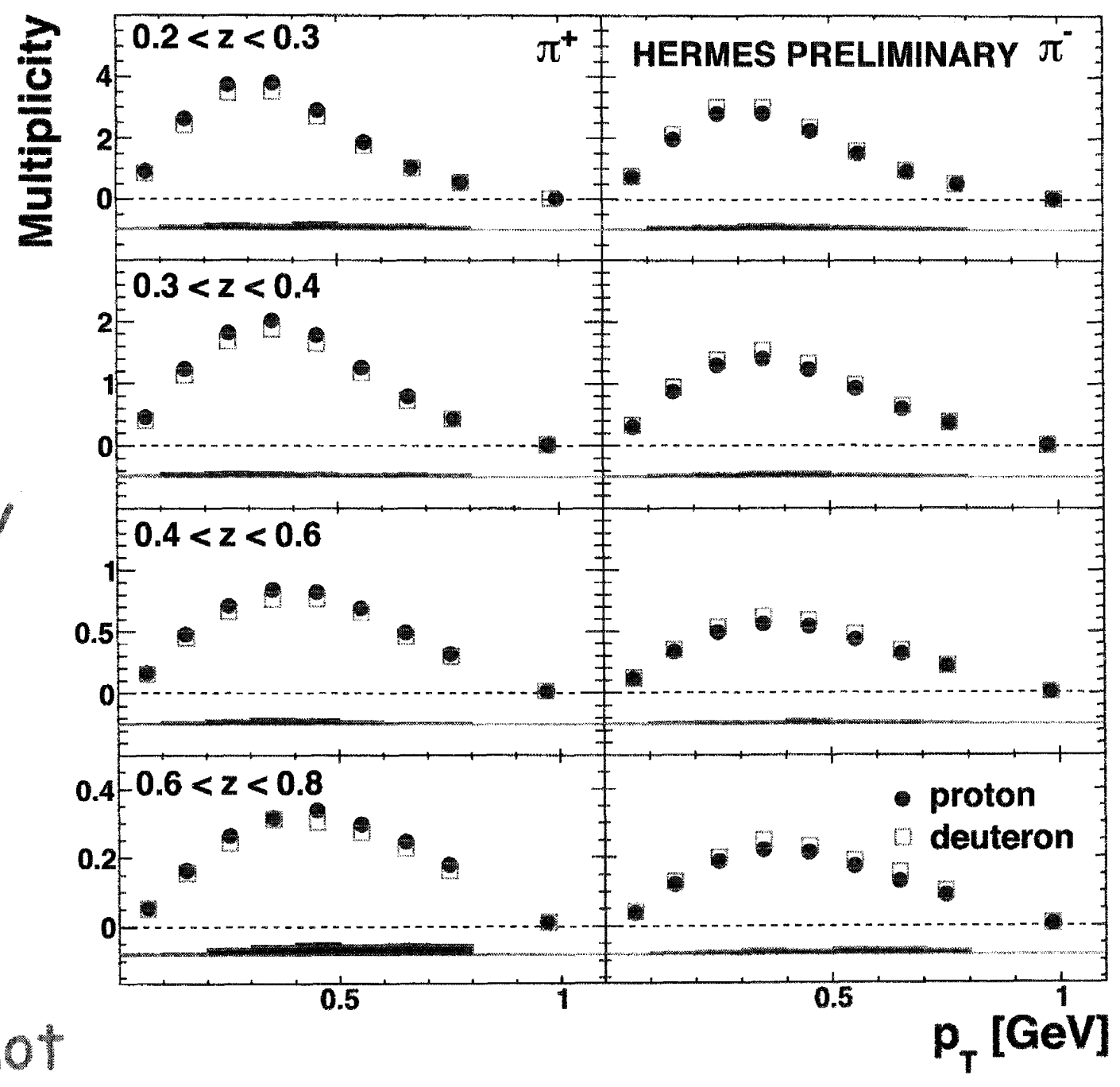
fragmentation functions not only binned in $z$ but also in $P_{h \perp}$ also available for kaons 


\begin{tabular}{|c|c|c|c|}
\hline & $\mathrm{U}$ & $\mathrm{L}$ & $\mathrm{T}$ \\
\hline $\mathrm{U}$ & $f_{1}$ & & $h_{\mathrm{L}}^{\perp}$ \\
\hline $\mathrm{L}$ & & $g_{1 L}$ & $h_{1}^{\mathrm{L}}$ \\
\hline $\mathrm{T}$ & $f_{1 T}^{\perp}$ & $g_{\mathrm{L}}$ & $h_{1} h_{1 T}^{\perp}$ \\
\hline
\end{tabular}

\section{Collins amplitudes COMPASS \& HERMES}

- different trend for $\mathrm{K}^{-}$

- opposite sign conventions!

- similar behavior for pions

- similar behavior for $\mathrm{K}^{+}$

COMPASS 2007 proton data

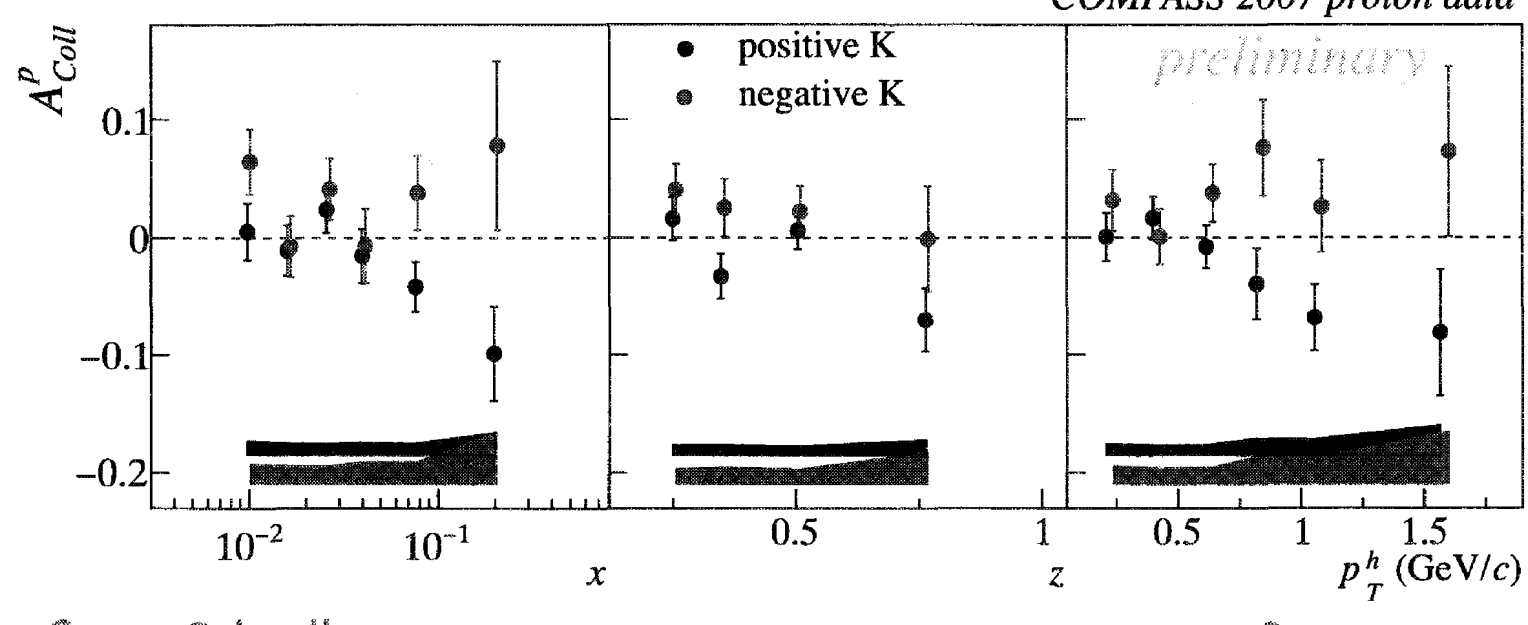

Gunar Schnell

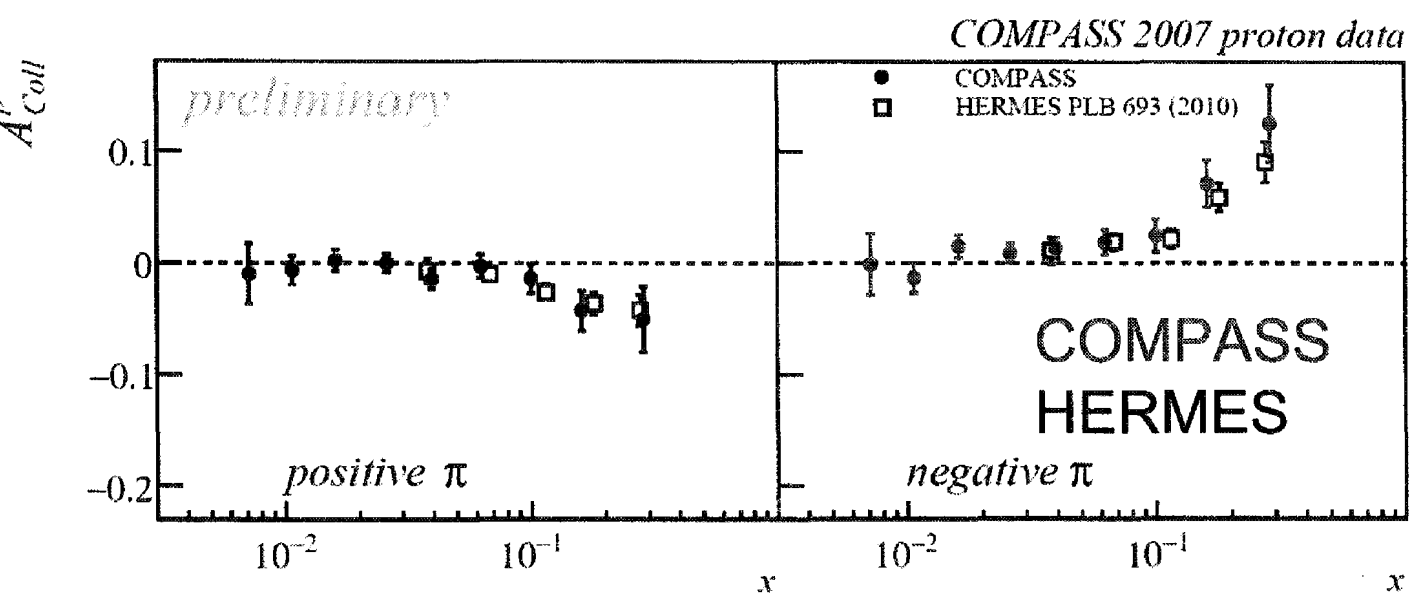

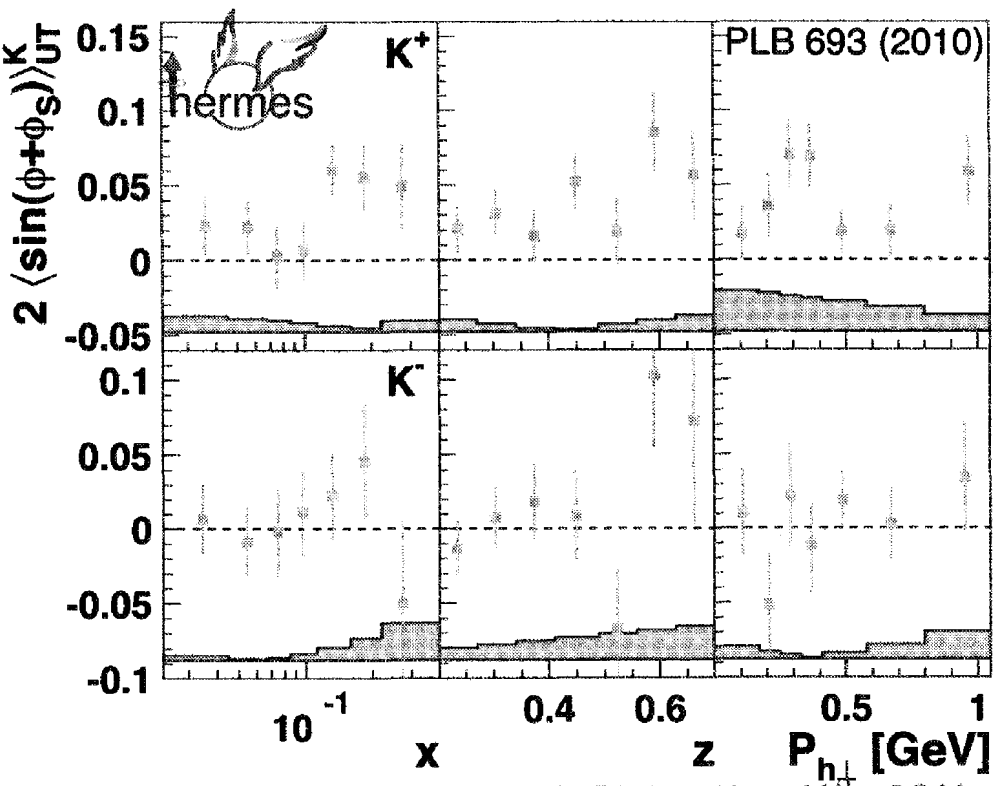

DY 2011, BNL - May 11\%,2011 


\begin{tabular}{|c|c|c|c|}
\hline & $\mathrm{U}$ & $\mathrm{L}$ & $\mathrm{T}$ \\
\hline $\mathrm{U}$ & $f_{1}$ & & $h_{\mathrm{L}}^{\perp}$ \\
\hline $\mathrm{L}$ & & $g_{1 L}$ & $h_{1 L}^{\perp}$ \\
\hline $\mathrm{T}$ & $f_{1 \mathrm{~L}}^{\perp}$ & $g_{1 T}$ & $h_{1}, h_{1 T}^{\perp}$ \\
\hline
\end{tabular}

Sivers amplitudes for pions $2\left\langle\sin \left(\phi-\phi_{S}\right)\right\rangle_{U_{\mathrm{T}}}=-\frac{\sum_{q} e_{q}^{2} f_{1 \mathrm{~T}}^{\perp, q}\left(x, p_{T}^{2}\right) \otimes \mathcal{W} D_{1}^{q}\left(z, k_{T}^{2}\right)}{\sum_{q} e_{q}^{2} f_{1}^{q}\left(x, p_{T}^{2}\right) \otimes D_{1}^{q}\left(z, k_{T}^{2}\right)}$

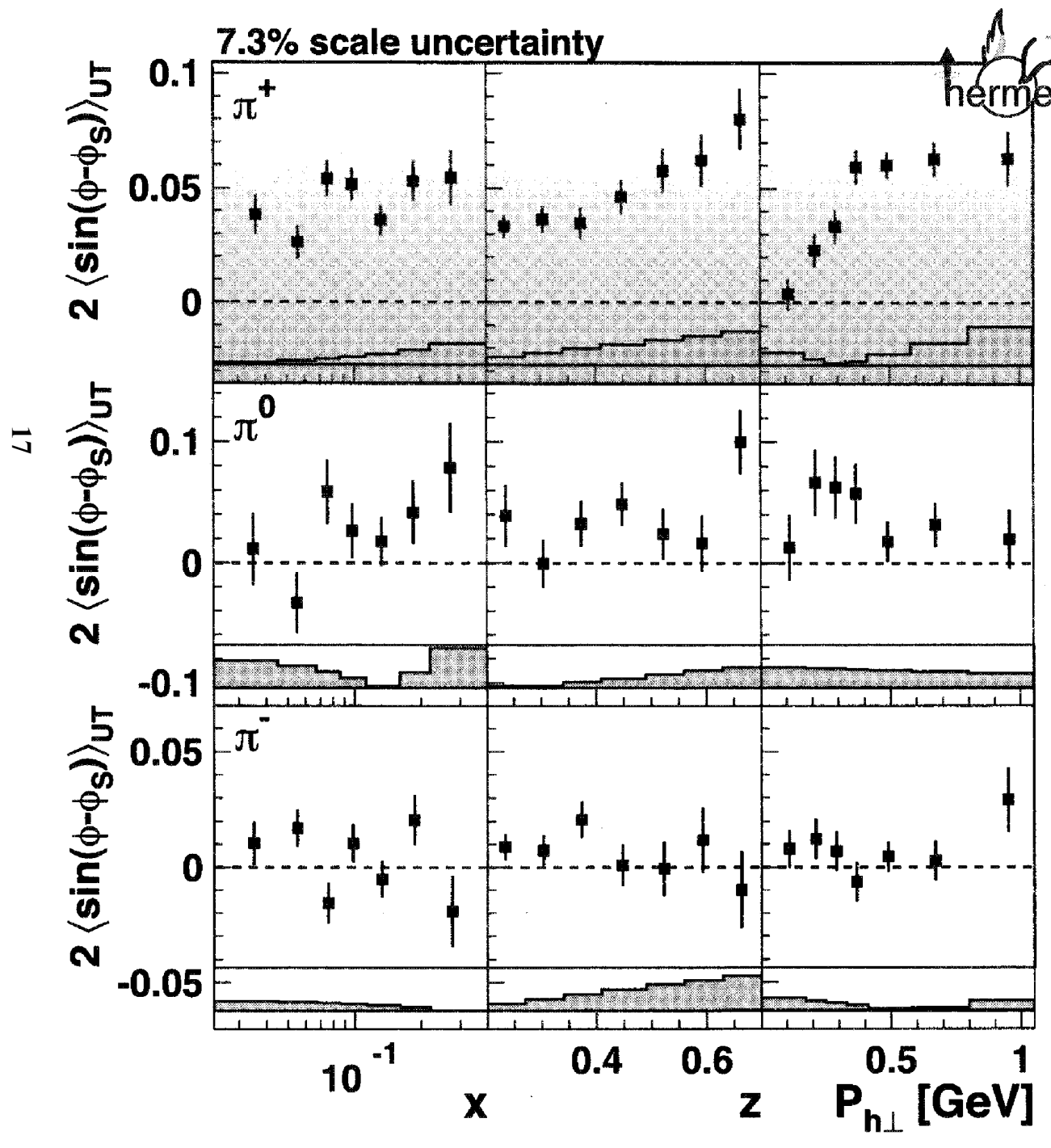

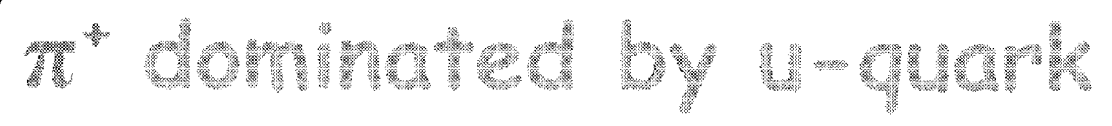
scontering:

$$
\frac{f_{1 T}^{1, u}\left(x, p_{T}^{2}\right) \otimes w D_{1}^{u \rightarrow \pi^{+}}\left(z, k_{T}^{2}\right)}{f_{1}^{u}\left(x, p_{T}^{2}\right) \otimes D_{1}^{u \rightarrow \pi^{+}}\left(z, k_{T}^{2}\right)}
$$

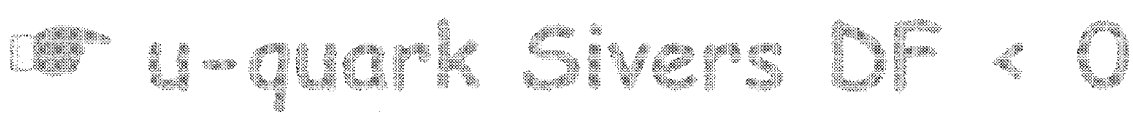

a-quark Sivers of > 0 (cancelation for $\pi^{-}$)

u-d cancelation supported by COMPASS D data 


\section{Summary}

- TMDs provide rich field for studying nucleon structure

- transversity is non-zero and quite sizable

- can be measured, e.g. via Collins effect or s-p interference in 2-hadron fragmentation

- Sivers and Boer-Mulders effects are also non-zero

- direct probe of "physics of the QCD gauge links"

- so far no sign of a non-zero Mulders-Tangerman (aka Pretzelosity) distribution

- but first evidence for non-vanishing worm-gear functions

- great opportunities for measurements in Drell-Yan especially for naive-T-odd TMDS 


\section{Status (critique) of TMD phenomenology}

The phenomenological study of TMDs and their extraction from experimental data is reviewed, with attention to possible sources of uncertainties. The role of TMDs in different processes - SIDIS, e+e- and

NN inclusive interactions - is discussed. Predictions and suggestions for Drell-Yan measurements are given.

Opportunities for Drell-Yan Physics at RHIC May 11-13, 2011, RIKEN BNL Mauro Anselmino, Torino University \& INFN 
simple Sivers functions for $u$ and $d$ quarks are sufficient to fit the available SIDIS data large and very small $x$ dependence not constrained by data talk by A. Prokudin

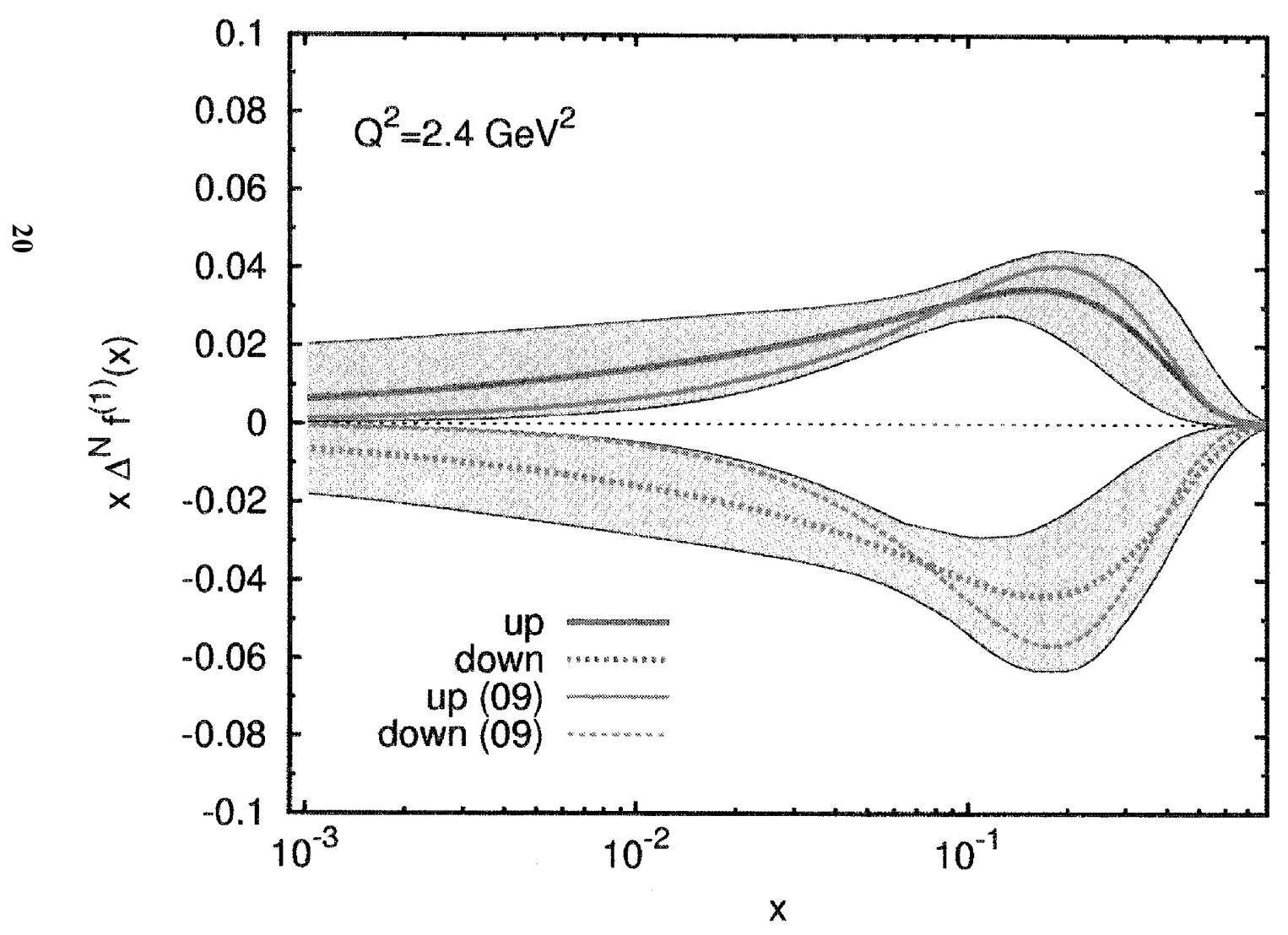

new and previous extraction of $u$ and $d$ Sivers functions

S. Melis and A. Prokudin, preliminary results

Anselmino et al. Eur. Phys. J. A39,89 (2009) 


\section{azimuthal modulations in TFR}

(M. A, V. Barone, A. Kotzinian, PL B699 (2011) 108)

cross section for lepto-production of an unpolarized or spinless hadron in the TFR

$\simeq$

$$
\begin{aligned}
& \frac{\mathrm{d} \sigma^{\mathrm{TFR}}}{\mathrm{d} x_{B} \mathrm{~d} y \mathrm{~d} \zeta \mathrm{d}^{2} \boldsymbol{P}_{h \perp} \mathrm{d} \phi_{S}}=\frac{2 \alpha_{\mathrm{em}}^{2}}{Q^{2} y}\left\{\left(1-y+\frac{y^{2}}{2}\right)\right. \\
& \left.\quad \times \sum_{a} e_{a}^{2}\left[M\left(x_{B}, \zeta, \boldsymbol{P}_{h \perp}^{2}\right)-\left|\boldsymbol{S}_{\perp}\right| \frac{\left|\boldsymbol{P}_{h \perp}\right|}{m_{h}} M_{T}^{h}\left(x_{B}, \zeta, \boldsymbol{P}_{h \perp}^{2}\right) \sin \left(\phi_{h}-\phi_{S}\right)\right)\right] \\
& \quad+\lambda_{l} y\left(1-\frac{y}{2}\right) \sum_{a} e_{a}^{2}\left[S_{\|} \Delta M_{L}\left(x_{B}, \zeta, \boldsymbol{P}_{h \perp}^{2}\right)\right. \\
& \left.\left.\quad+\left|\boldsymbol{S}_{\perp}\right| \frac{\left|\boldsymbol{P}_{h \perp}\right|}{m_{h}} \Delta M_{T}^{h}\left(x_{B}, \zeta, \boldsymbol{P}_{h \perp}^{2}\right) \cos \left(\phi_{h}-\phi_{S}\right)\right]\right\} .
\end{aligned}
$$

possible Sivers-like azimuthal dependence from target fragmentation region 


\section{sign mismatch}

(Kang, Qiu, Vogelsang, Yuan)

$$
g T_{q, F}(x, x)=-\left.\int d^{2} k_{\perp} \frac{\left|k_{\perp}\right|^{2}}{M} f_{1 T}^{\perp q}\left(x, k_{\perp}^{2}\right)\right|_{\text {SIDIS }}
$$

as extracted from fitting $A_{N}$ data, with that obtained by $\approx$ inserting in the the above relation the SIDIS extracted Sivers functions similar magnitude, but opposite sign!

the same mismatch does not occurr adopting TMD factorization; the reason is that the hard scattering part in higher-twist factorization is negative 


\section{Cahn effect in unpolarized D-Y}

M. Boglione, S. Melis, arXiv:1103.2084

\section{access to $\left\langle k_{\perp}^{2}\right\rangle$}

$$
\begin{gathered}
\frac{d \sigma^{u n p}}{d^{4} q d \Omega^{\prime}}=\frac{\alpha^{2}}{6 M^{2} s} \sum_{q} e_{q}^{2} f_{a / A}^{q}\left(x_{a}\right) \bar{f}_{b / B}^{q}\left(x_{b}\right) \frac{e^{-q_{T}^{2} /\left\langle q_{T}^{2}\right\rangle}}{\pi\left\langle q_{T}^{2}\right\rangle}\{\left(1+\cos ^{2} \theta^{\prime}\right)+\underbrace{\frac{q_{T}}{M} \frac{\left\langle k_{\perp a}^{2}\right\rangle-\left\langle k_{\perp b}^{2}\right\rangle}{\left\langle q_{T}^{2}\right\rangle} \sin 2 \theta^{\prime} \cos \phi^{\prime}}_{\text {Cahn effect }}\} \\
\left\langle k_{\perp a}^{2}\right\rangle+\left\langle k_{\perp b}^{2}\right\rangle \equiv\left\langle q_{T}^{2}\right\rangle \quad \boldsymbol{q}_{T}=\boldsymbol{k}_{\perp a}+\boldsymbol{k}_{\perp b} \quad \\
f_{a / A}\left(x_{a}, k_{\perp a}\right)=f_{a / A}\left(x_{a}\right) \frac{e^{-k_{\perp a}^{2} /\left\langle k_{\perp a}^{2}\right\rangle}}{\pi\left\langle k_{\perp a}^{2}\right\rangle} \\
\text { gaussian } k_{\perp} \text { dependence } \\
\text { no effect if }\left\langle k_{\perp a}^{2}\right\rangle=\left\langle k_{\perp b}^{2}\right\rangle
\end{gathered}
$$

same conclusion holds for non gaussian distributions 


\section{Predictions for $A_{N}$}

\section{Sivers functions as extracted from SIDIS data, with opposite sign}
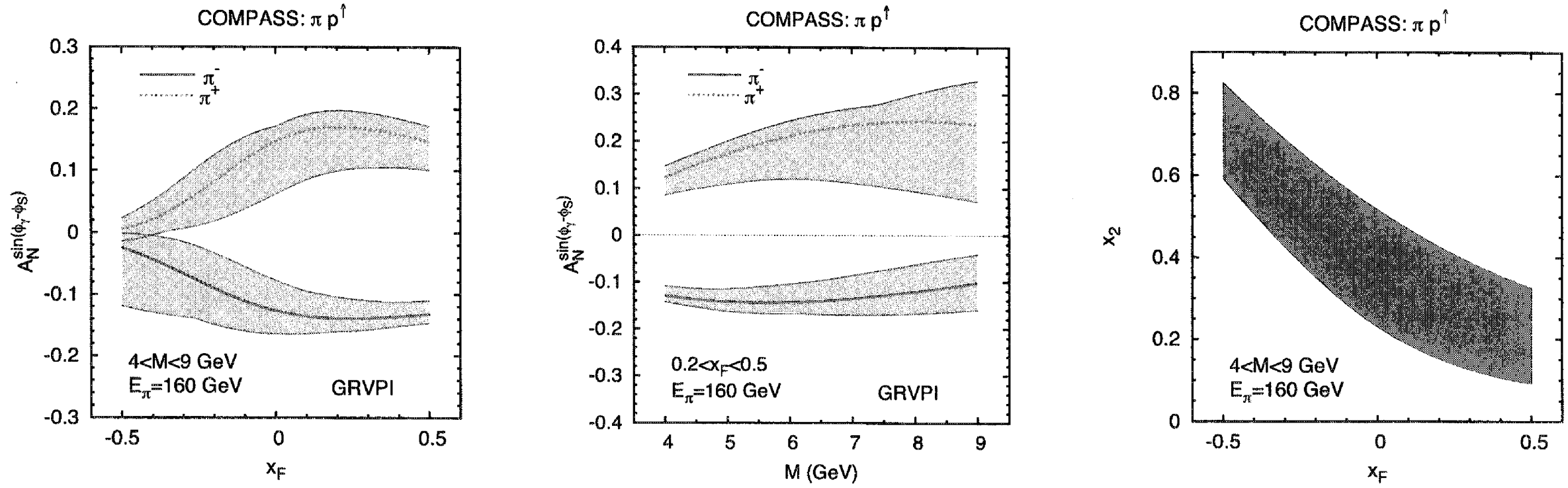

$\stackrel{n}{N}$
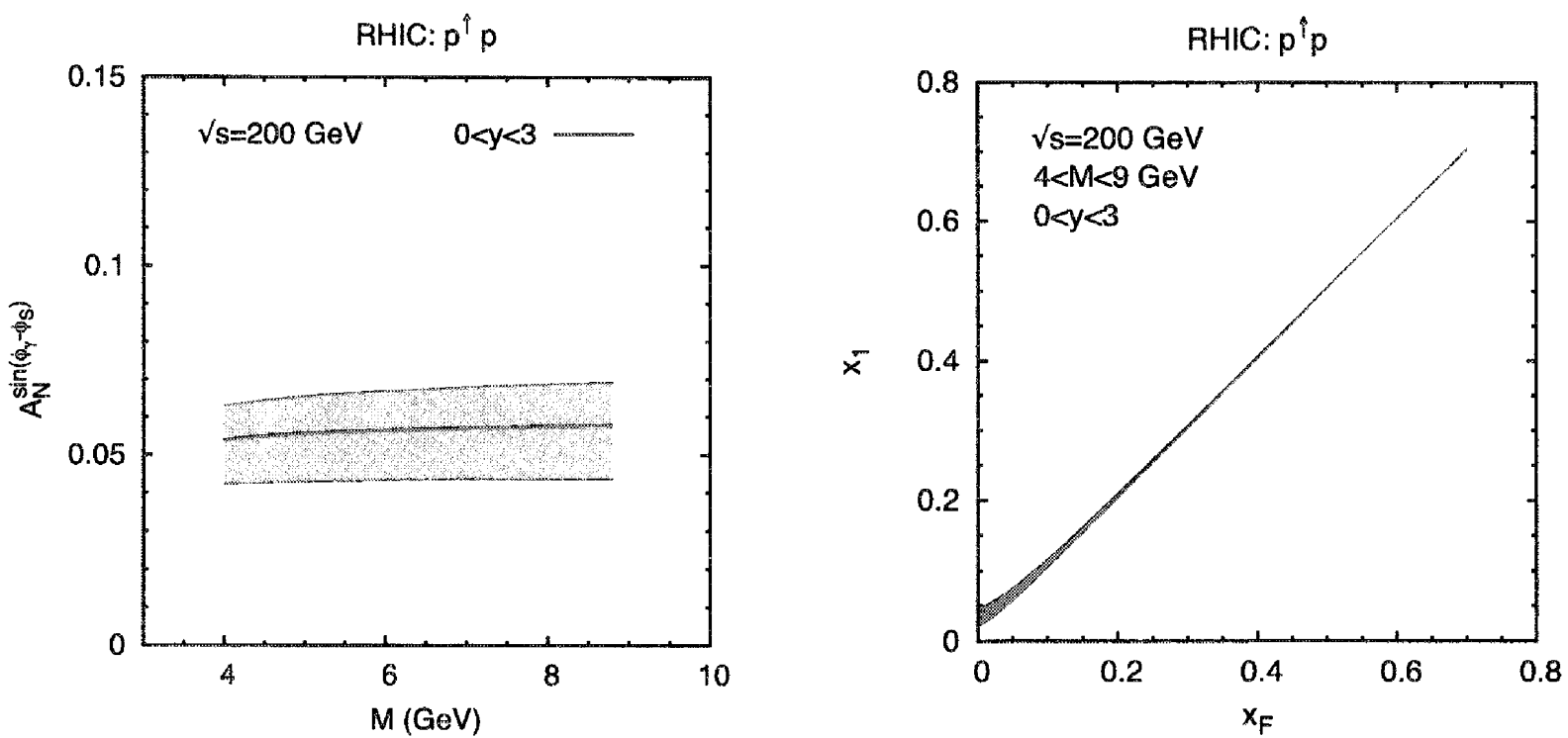

M.A., M. Boglione, U. D'Alesio, S. Melis, F. Murgia, A. Prokudin, e-Print: arXiv:0901.3078 


\section{Sivers effect: from SIDIS to pp}

\section{- sign change and sign mismatch}

\section{Zhongbo Kang}

\section{RIKEN BNL Research Center, Brookhaven National Laboratory}

Abstract: The \$k_ Iperp\$-moment of a quark's Sivers function is known to be related to the corresponding twist three quark-gluon correlation function $\$ T,\{q, F\}(x, x) \$$. The two functions have been extracted from data for single-spin asymmetries in semi-inclusive deep inelastic scattering and in single-inclusive hadron production in \$pp\$ collisions, respectively. Performing a consistent comparison of the extracted functions, we find that they show a "sign mismatch": while the magnitude of the functions is roughly consistent, the \$K_ \perp\$-moment of the Sivers function has opposite sign from that of $\$ T$ _ $q, F\}(x, x) \$$, both for up and for down quarks. Barring any inconsistencies in our theoretical understanding of the Sivers functions and their process dependence, the implication of this mismatch is that either, the Sivers effect is not dominanty responsible for the observed single-spin asymmetries in \$pp\$ collisions or, the current semiinclusive lepton scattering data do not sufficiently constrain the \$ $\$$ _. lperp\$-moment of the quark Sivers functions. Both possibilities strengthen the case for further experimental investigations of single-spin asymmetries in high-energy \$pp\$ and \$ep\$ scattering. 


\section{Time-reversal modified universality of the Sivers function}

- Different gauge link for gauge-invariant TMD distribution in SIDIS and $\begin{aligned} & \text { DY } \\ & f_{q / h} \uparrow\left(x, \mathbf{k}_{\perp}, \vec{S}\right)\end{aligned}=\int \frac{d y^{-} d^{2} y_{\perp}}{(2 \pi)^{3}} e^{i x p^{+} y^{-}-i \mathbf{k}_{\perp} \cdot \mathbf{y}_{\perp}}\langle p, \vec{S}| \bar{\psi}\left(0^{-}, 0_{\perp}\right)$ Gauge link $\frac{\gamma^{+}}{2} \psi\left(y^{-}, \mathbf{y}_{\perp}\right)|p, \vec{S}\rangle$

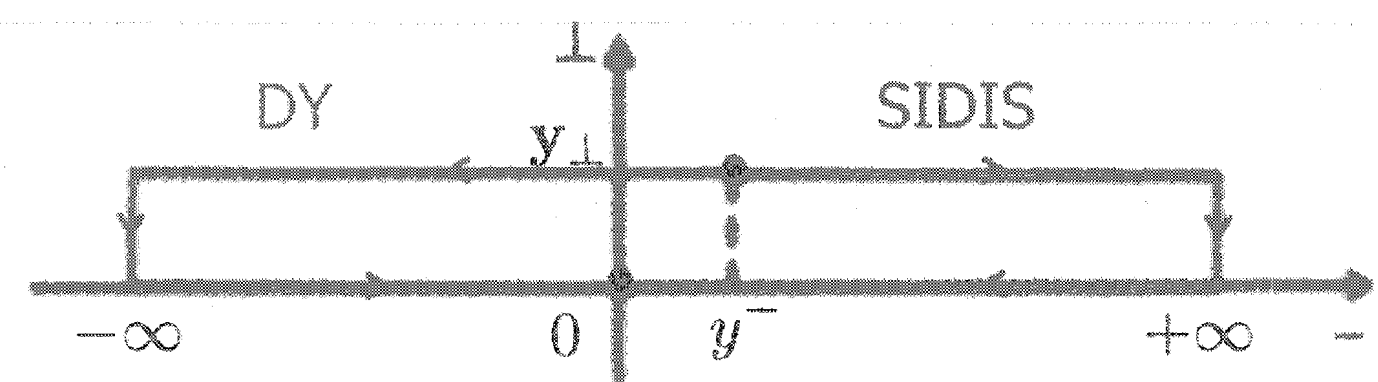

న

$$
\text { Wilson Loop } \sim \exp \left[-i g \int_{\Sigma} d \sigma^{\mu \nu} F_{\mu \nu}\right] \quad \text { Area is NOT zero }
$$

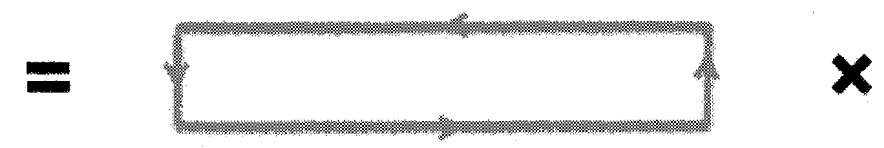

m Parity and time-reversal invariance:

$$
\Delta^{N} f_{q / h^{\uparrow}}^{\text {SIDIS }}\left(x, k_{\perp}\right)=-\Delta^{N} f_{q / h^{\uparrow}}^{\mathrm{DY}}\left(x, k_{\perp}\right)
$$

Most critical test for TMD approach to SSA 


\section{What about the connections?}

- Both seem to describe the data well (in their own kinematic region), but what about their connections?

- At the operator level, ETQS function is related to the first kt-moment of the Sivers function

ก

$$
g T_{q, F}(x, x)=-\left.\int d^{2} k_{\perp} \frac{\left|k_{\perp}\right|^{2}}{M} f_{1 T}^{\perp q}\left(x, k_{\perp}^{2}\right)\right|_{\text {SIDIS }}
$$

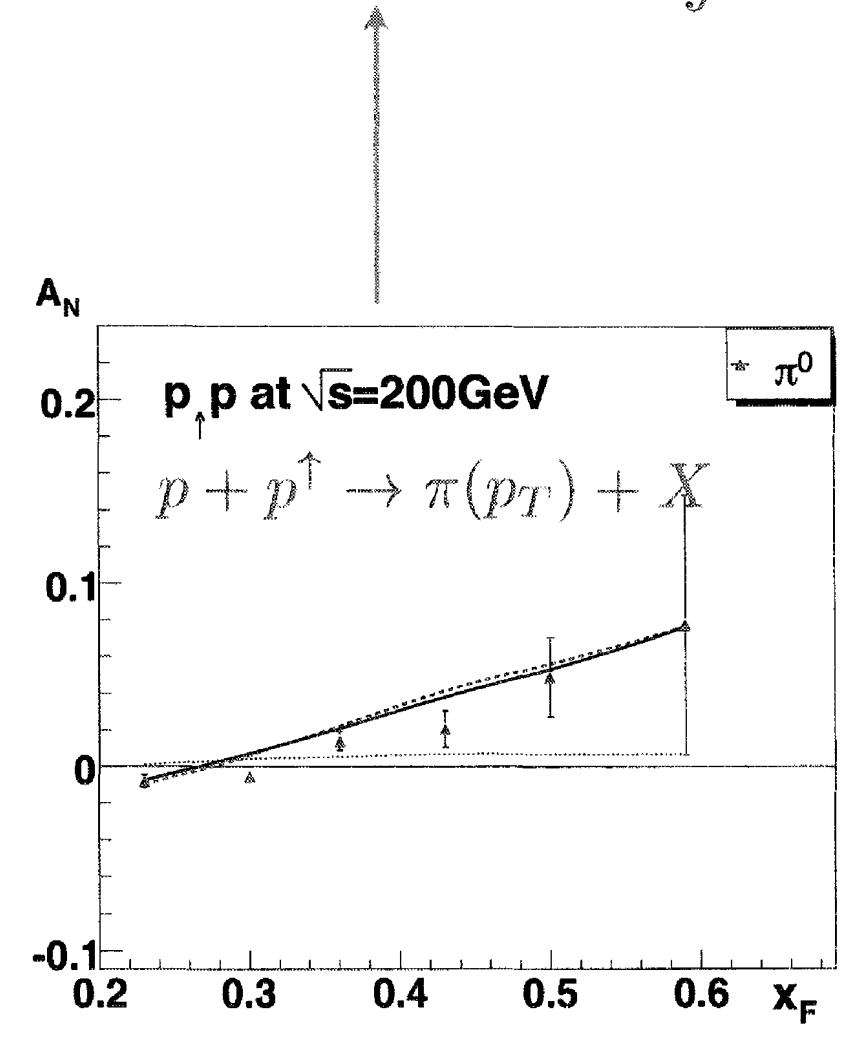

May 11, 2011

Zhongbo Kang, RBRC/BNL

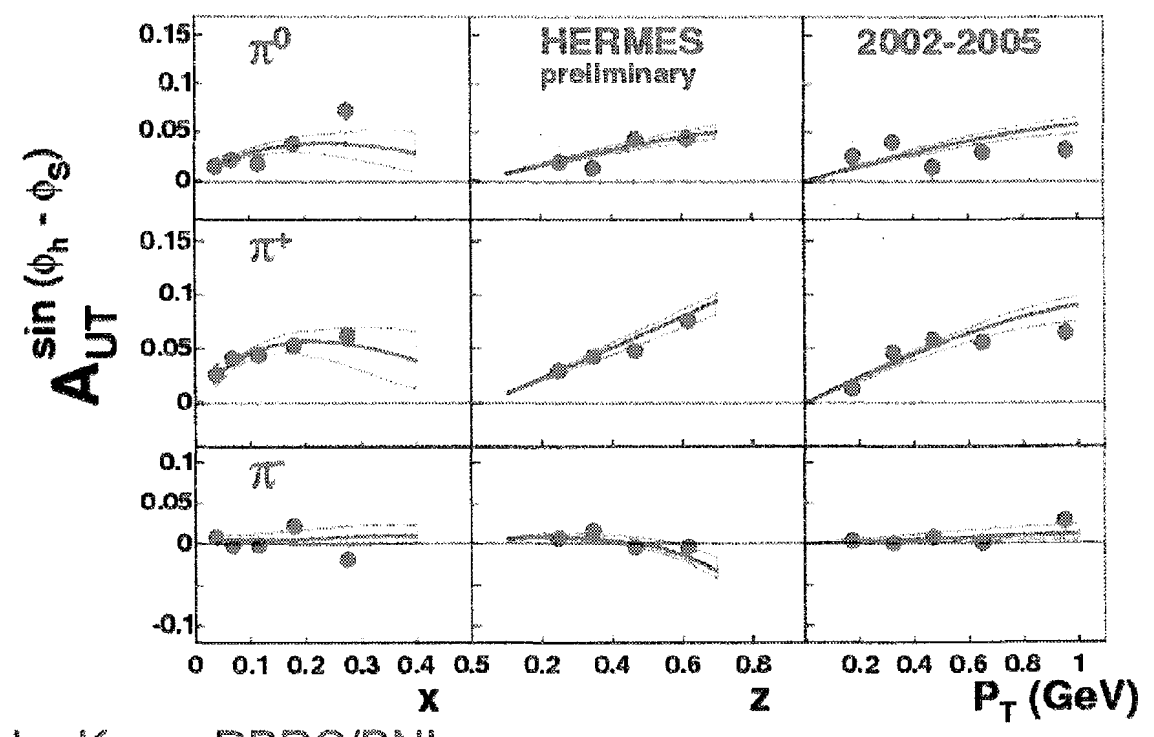




\section{Initial- and final-state interaction in pp collisions}

- The dominant channel is qg $\rightarrow \mathrm{qg}$
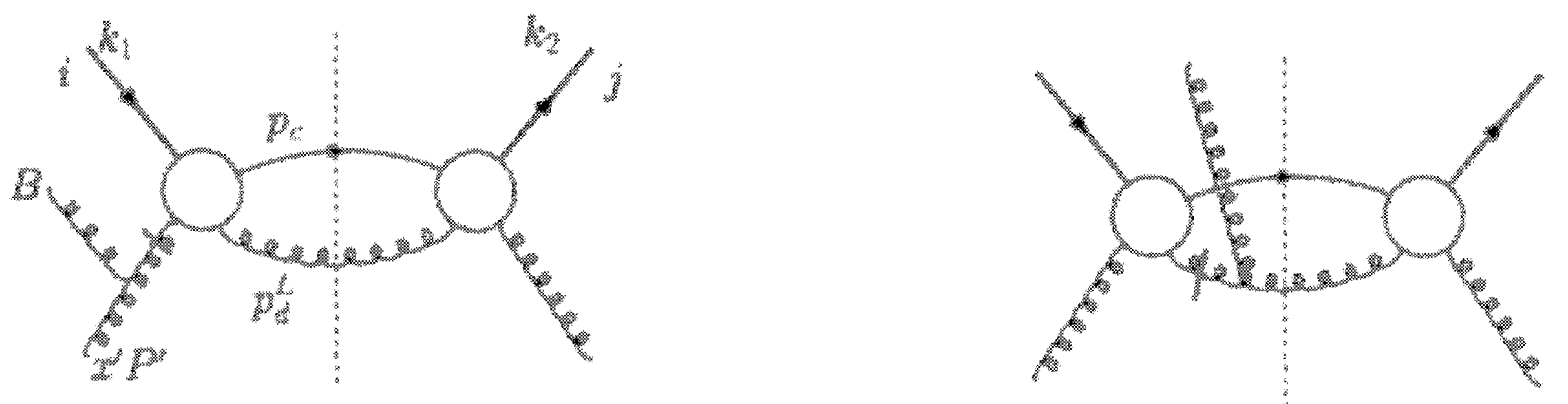

$\because \quad H_{q g \rightarrow q g}^{U}=\frac{N_{c}^{2}-1}{2 N_{c}^{2}}\left[-\frac{\hat{s}}{\hat{u}}-\frac{\hat{u}}{\hat{s}}\right]\left[1-\frac{2 N_{c}^{2}}{N_{c}^{2}-1} \frac{\hat{s} \hat{u}}{\hat{t}^{2}}\right] \stackrel{|\hat{t}| \ll \hat{s} \sim|\hat{u}|}{\longrightarrow}\left[\frac{2 \hat{s}^{2}}{\hat{t}^{2}}\right]$

$$
\begin{aligned}
& H_{q g \rightarrow q g}^{I}=\frac{1}{2\left(N_{c}^{2}-1\right)}\left[-\frac{\hat{s}}{\hat{u}}-\frac{\hat{u}}{\hat{s}}\right]\left[1-N_{c}^{2} \frac{\hat{u}^{2}}{\hat{t}^{2}}\right] \stackrel{|\hat{t}| \ll \hat{s} \sim|\hat{u}|}{\longrightarrow}\left(-\frac{N_{c}^{2}}{2\left(N_{c}^{2}-1\right)}\right]\left[\frac{2 \hat{s}^{2}}{\hat{t}^{2}}\right] \\
& H_{q g \rightarrow q g}^{F}=\frac{1}{2 N_{c}^{2}\left(N_{c}^{2}-1\right)}\left[-\frac{\hat{s}}{\hat{u}}-\frac{\hat{u}}{\hat{s}}\right]\left[1+2 N_{c}^{2} \frac{\hat{s} \hat{u}}{\hat{t}^{2}}\right] \stackrel{|\hat{t}| \ll \hat{s} \sim|\hat{u}|}{\longrightarrow}\left[-\frac{1}{N_{c}^{2}-1}\right]\left[\frac{2 \hat{s}^{2}}{\hat{t}^{2}}\right]
\end{aligned}
$$

- Sivers effect in single hadron production is more similar to DY 


\section{Directly obtained ETQS function}

- ETQS function could be directly obtained from the global fitting of inclusive hadron production in hadronic collisions
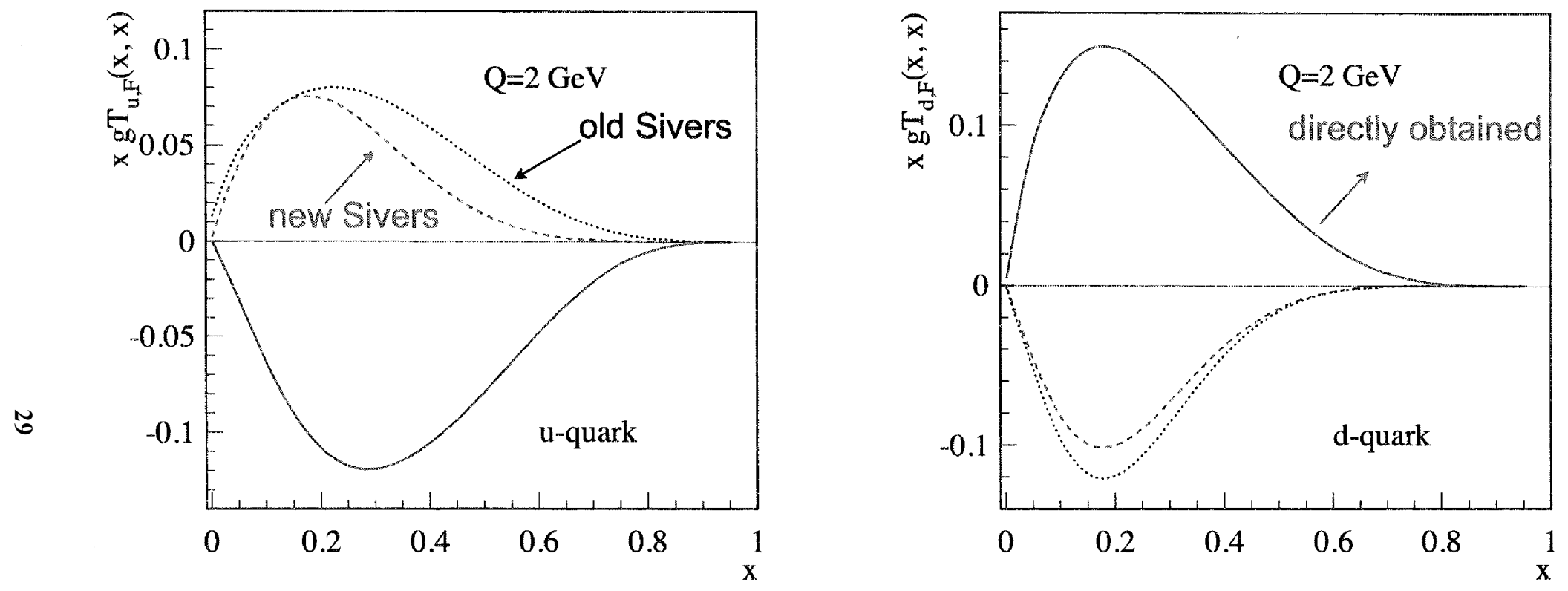

- directly obtained ETQS functions for both $u$ and d quarks are opposite in sign to those indirectly obtained from the kt-moment of the quark Sivers function "a sign mismatach" 


\section{Predictions for jet and direct photon}

- at RHIC 200 GeV:

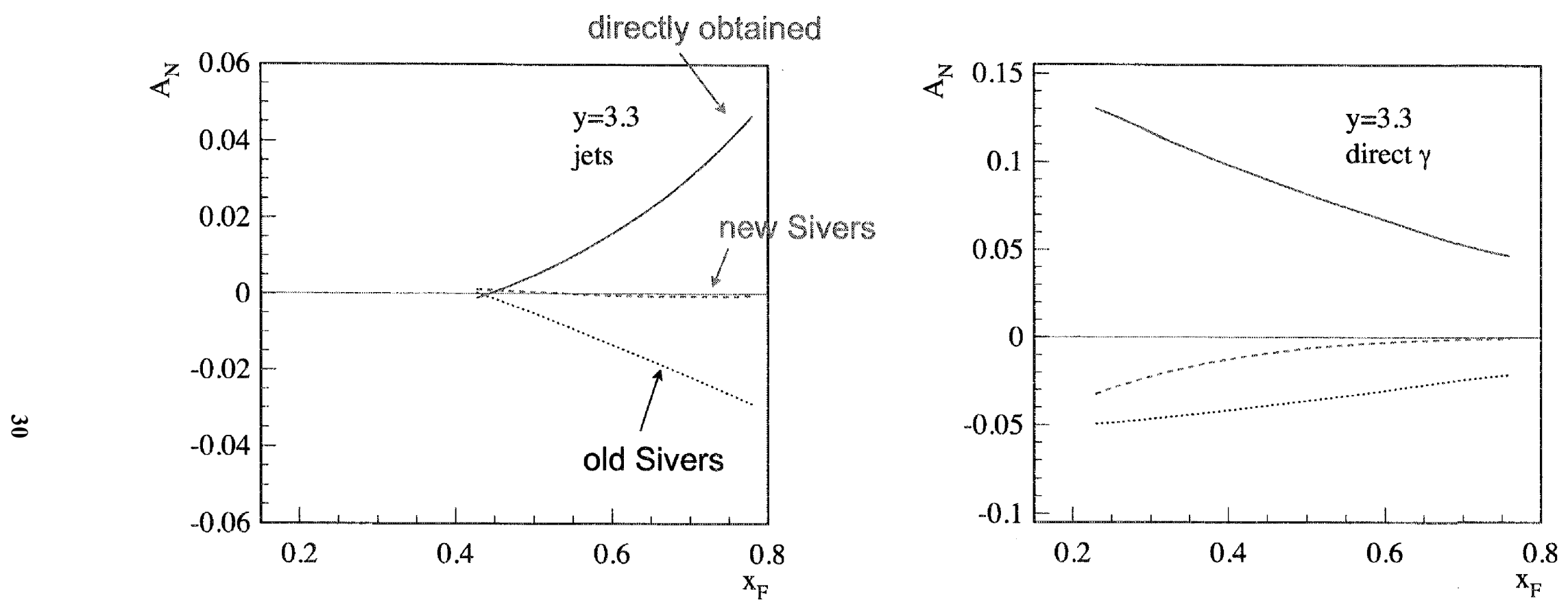


Gluon Densities and Dihadron correlations

Bo-Wen Xiao, Penn State

- In this talk, a complete calculation of dijet production in various processes is presented. It is well-known that there are two fundamental unintegrated gluen distributions in high density (CD. The first unintegrated gluon distribution, which measures the number density of

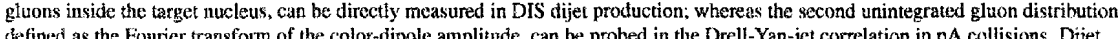
production cross section in $\mathrm{A}$ collision dependy on anth production cross section in $\mathrm{p} A$ collsion depends on both gluon distributions through convolutions. We conduct two independent
calculations (one is in CCC formalism and the other uses TMD approach.) for all of above processes. We find these two calculation agree perfectly. These calculation has shown important impact on the present RHIC and future EIC and I HeC. In the end i also present a comprehensive comparison between our numerical results and the forward dihadron production data measured by STAR.

Drell-Yan workshop at BNL, May, 2011 
DIS dijet correlation

Azimuthal angle correlation of dijet in DIS probes the WW gluon distributions
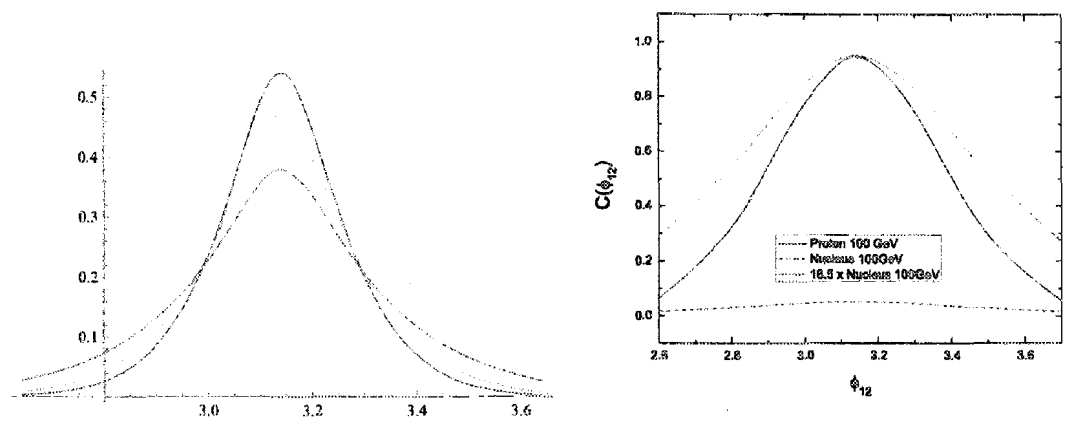

Remarks:

- $k_{1 \perp}=5.5 \mathrm{Gev}, k_{2 \perp}=5.0 \mathrm{Gev}$ and $Q_{s}^{2}=1,1.5,3 \mathrm{GeV}$

- Only away side peak is plotted due to the correlation limit.

- Suppression of away side peak and increase of width at large $Q_{s}^{2}$.

- Dramatic change between ep and eA collisions. $Q^{2}=4 \mathrm{GeV}^{2}, z_{11}=z_{2}=0.3$ PENASTAT $2 \mathrm{GeV}<p_{1 \perp}<3 \mathrm{GeV}$ and $1 \mathrm{GeV}<p_{2 \perp}<2 \mathrm{GeV}$.

- No pedestal. 


\section{Dilepton Pair + hadron correlation}

[F. Domingue\%, $\mathrm{B} \times$ and $\mathrm{F}$. Yan, in preparation]

Azimuthal angle correlation of $\gamma^{*}+\pi^{0}$ at forward rapidity 3.2 :
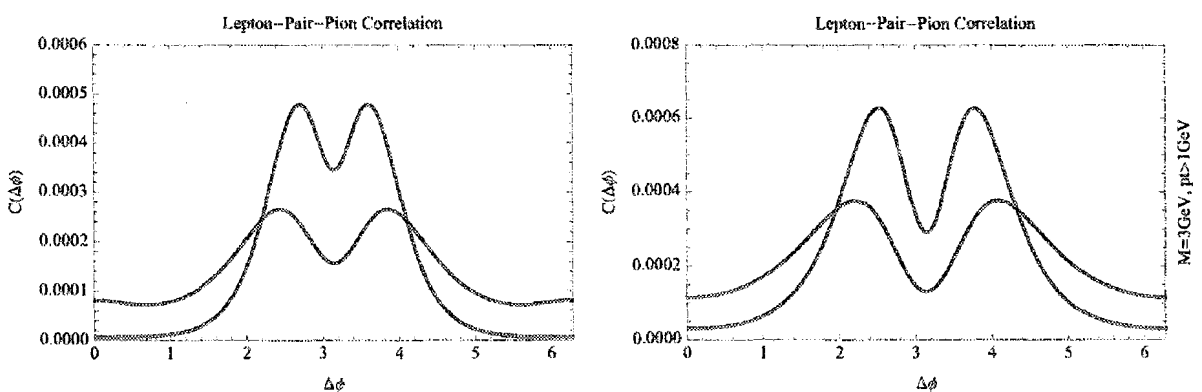

\section{Remarks:}

$p_{1 \perp}>1.5 \mathrm{Gev}, p_{2 \perp}>1.5 \mathrm{Gev}$ and $M^{2}=1 \mathrm{GeV}^{2}$

- $p_{1 \perp}>1 \mathrm{Gev}, p_{2 \perp}>1 \mathrm{Gev}$ and $M^{2}=9 \mathrm{GeV}^{2}$;

- Suppression of away side peak at central $d A u$ collisions.

Double peak structure on the away side comes from the fact that $x G^{(2)} \propto q_{\perp}^{2}$ in the small $q_{\perp}$ limit. 


\section{Gluon+quark jets correlation}

Including all the $q g \rightarrow q g, r g-i g$ and $g, \cdots \rightarrow g$ channels, a lengthy calculation gives

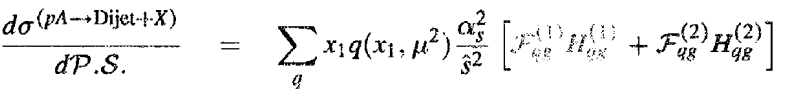

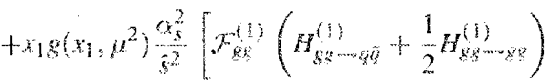

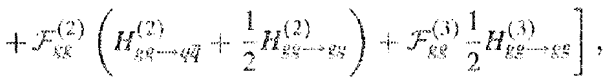

with the various gluon distributions defined as

$$
\begin{aligned}
F_{q k}^{(1)} & =x G^{(2)}\left(x, q_{\perp}\right), F_{q g}^{(2)}=\int x G^{(1)} \otimes F, \\
F_{g g}^{(1)} & =\int x G^{(2)} \otimes F, F_{g}^{(2)}=\cdots \int \frac{q_{1 \perp} \cdot q_{2 \perp}}{q_{1 \perp}^{2}} x G^{(2)} \otimes F, \\
F_{g}^{(3)} & =\int x G^{(1)}\left(q_{1}\right) \otimes F \otimes F,
\end{aligned}
$$

where $F=\int \frac{d^{2} r_{\perp}}{(2 \pi)^{2}} e^{-i q_{\perp} \cdot r_{\perp}} \frac{1}{\hat{N}_{c}}\left\langle\operatorname{Tr} U\left(r_{\perp}\right) U^{\dagger}(0)\right\rangle_{x_{g}}$. Remarks: Only the first term was known before. 


\section{Comparing to STAR data including both $q+g$ and $g+g$}

[A. Stasio, BX. F. Yuan, in preparation]

For away side peak in both peripheral and central $d A u$ collisions:

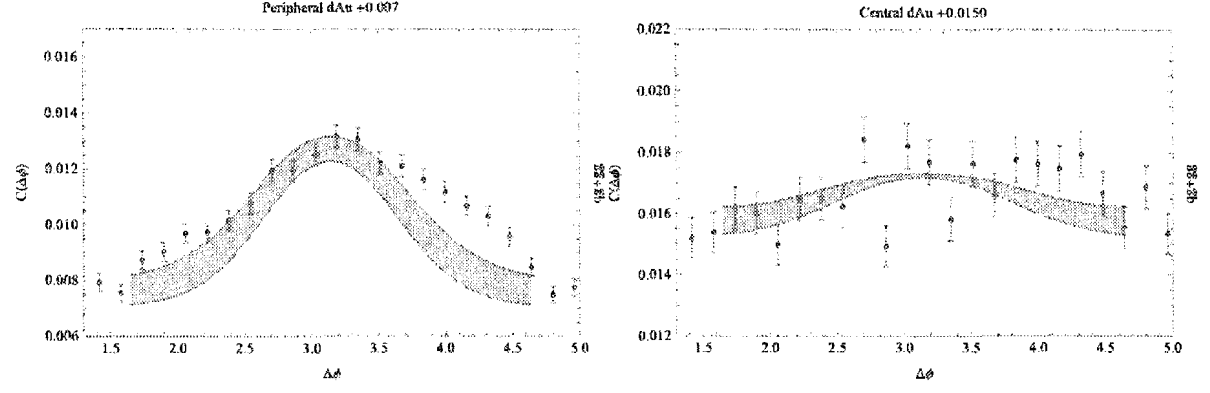

Adding a $k$-factor of 2 to the ratio since the total single inclusive cross section is twice of the data at $\eta=3.2$.

- Other parameters are kept the same. 


\section{Comparing to STAR data including both $q+g$ and $g+g$}

[A. Stasto, BX, Ka Yun, in preparation]

For minimum bias away side peak in $d A u$ collisions in $q+g$ channel:

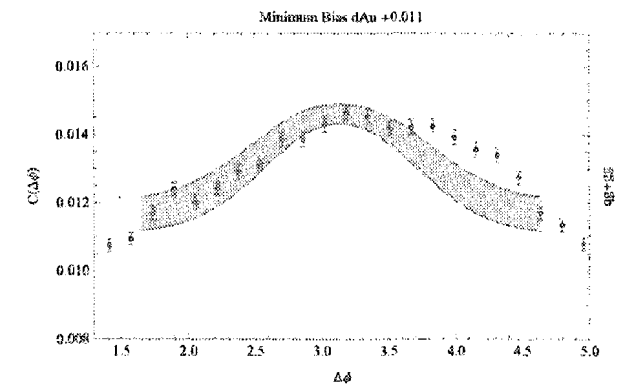

Adding a $k$ - factor of 2 to the ratio since the total single inclusive cross section is twice of the data at $\eta=3.2$.

Peripheral $b=6.8 \pm 1.7 \mathrm{~m}$ with $c(b)=0.45$ and width $\sigma \simeq 0.99$;

- Central $b=2.7 \pm 1.3 \mathrm{fm}$ with $c(b)=0.85$ and width $\sigma \simeq 1.6$

- Minimum Bias $c(b)=0.56 \Rightarrow(b)=6 \mathrm{fm}$ and width $\sigma \simeq 1.2$. 


\section{Jamal Jalilian-Marian}

\section{Baruch College, New York NY 10010}

In the Color Glass Condensate formalism, the amplitudes for quark anti-quark production in DIS and virtual photon (DY) production

$\circlearrowleft$ in proton (deuteron)-nucleus (pA) collisions are related via crossing symmetry. Both production cross sections involve only the dipole (two-point function of Wilson lines) function. Therefore knowledge of the dipole profile gained in DIS structure function studies can be used to predict dilepton production in pA collisions. Lam-Tung relation between the DY structure functions is shown to be sensitive to the high gluon density effects at small $\mathrm{x}$.

*based on work done in collaboration with F. Gelis 


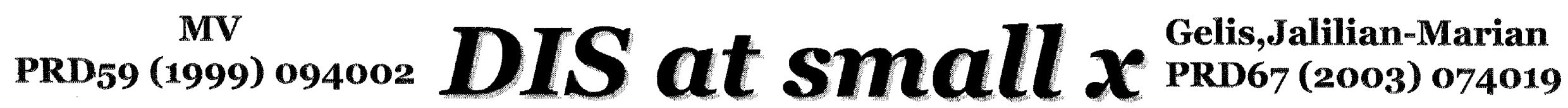
consider $\gamma^{\star} \mathbf{T} \rightarrow \mathbf{q} \overline{\mathbf{q}} X$

$$
\begin{aligned}
M^{\mu}(k ; q, p)= & \frac{i}{2} \int \frac{d^{2} l_{t}}{(2 \pi)^{2}} d^{2} x_{t} d^{2} y_{t} e^{i l_{t} \cdot x_{t}} e^{i\left(p_{t}+q_{t}-k_{t}-l_{t}\right) \cdot y_{t}} \bar{u}(q) \Gamma^{\mu}(k ; q, p) v(p) \\
& {\left[V\left(x_{t}\right) V^{\dagger}\left(y_{t}\right)-1\right] }
\end{aligned}
$$

cross section:

$\infty$

$$
2 \mathbf{p}_{0} 2 \mathbf{q}_{0} \frac{\mathbf{d} \sigma}{\mathbf{d}^{3} \mathbf{q} \mathbf{d}^{3} \mathbf{p}}=\frac{1}{(2 \pi)^{5}} \frac{1}{\mathbf{k}^{-}} 2 \pi \delta\left(\mathbf{k}^{-} \mathbf{p}^{-}-\mathbf{q}^{-}\right)\left\langle\mathbf{M}^{\mu} \mathbf{M}^{\nu \star}\right\rangle \epsilon_{\mu}(\mathbf{k}) \epsilon_{\nu}^{\star}(\mathbf{k})
$$

to get the DIS total cross section, integrate over quark, anti-quark momenta

$$
\begin{aligned}
& \sigma^{\gamma^{\star} \mathbf{T} \rightarrow \mathbf{X}}=\int_{0}^{1} \mathrm{dz} \int \mathrm{d}^{2} \mathbf{x}_{\mathbf{t}} \mathrm{d}^{2} \mathbf{y}_{\mathbf{t}}|\Psi|^{2} \frac{1}{N_{c}}\left\langle\operatorname{Tr}\left[1-V\left(\mathbf{x}_{t}\right) \mathbf{V}^{\dagger}\left(\mathbf{y}_{\mathbf{t}}\right)\right]\right\rangle \\
& \mathbf{T}\left(\mathbf{x}_{\mathrm{g}}, \mathbf{r}_{\mathrm{t}}, \mathbf{b}_{\mathrm{t}}\right)
\end{aligned}
$$




\section{DYat small $x$}

$\mathbf{q} \mathbf{T} \rightarrow \mathbf{q} \gamma^{\star} \mathbf{X}$
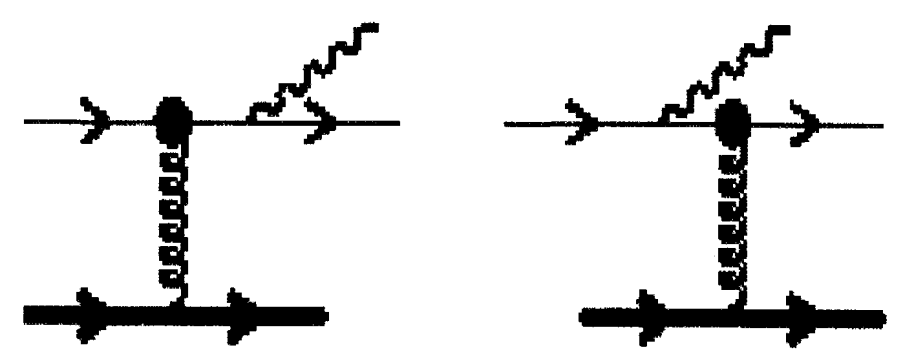

$\mathbf{M}^{\mu}(\mathbf{p} ; \mathbf{k}, \mathbf{q})=\mathbf{i} \int \mathbf{d}^{2} \mathbf{x}_{\mathbf{t}} \mathbf{e}^{\mathbf{i}\left(\mathbf{q}_{\mathbf{t}}+\mathbf{k}_{\mathbf{t}}-\mathbf{p}_{\mathbf{t}}\right) \cdot \mathbf{x}_{\mathbf{t}}} \overline{\mathbf{u}}(\mathbf{q}) \overline{\boldsymbol{\Gamma}}^{\mu}(\mathbf{k} ; \mathbf{q}, \mathbf{p}) \mathbf{u}(\mathbf{p})\left[\mathbf{V}\left(\mathbf{x}_{\mathbf{t}}\right)-\mathbf{1}\right]$

$$
\begin{aligned}
= & \frac{i}{2} \int \frac{d^{2} l_{t}}{(2 \pi)^{2}} d^{2} x_{t} d^{2} y_{t} e^{i l_{t} \cdot x_{t}} e^{i\left(q_{t}+k_{t}-p_{t}-l_{t}\right) \cdot y_{t}} \bar{u}(q) \Gamma^{\mu}(-k ; q,-p) u(p) \\
& {\left[V\left(x_{t}\right) V^{\dagger}\left(y_{t}\right)-1\right] \underbrace{V}_{V\left(y_{t}\right)} }
\end{aligned}
$$

น్ర extra: unitary matrix

cross section

$$
\begin{aligned}
\frac{d \sigma}{d z d^{2} k_{t} d \log M^{2} d^{2} b_{t}}= & \frac{2 \alpha_{e m}^{2}}{3 \pi} \int \frac{d^{2} l_{t}}{(2 \pi)^{4}} d^{2} r_{t} e^{i l_{t} \cdot r_{t}} T\left(x_{g}, b_{t}, r_{t}\right)\{ \\
& {\left[\frac{1+(1-z)^{2}}{z}\right] \frac{z^{2} l_{t}^{2}}{\left[k_{t}^{2}+(1-z) M^{2}\right]\left[\left(k_{t}-z l_{t}\right)^{2}+(1-z) M^{2}\right]} } \\
- & \left.z(1-z) M^{2}\left[\frac{1}{\left[k_{t}^{2}+(1-z) M^{2}\right]}-\frac{1}{\left[\left(k_{t}-z l_{t}\right)^{2}+(1-z) M^{2}\right]}\right]^{2}\right\}
\end{aligned}
$$

Gelis,Jalilian-Marian

PRD66 (2002) 094014 


\section{Evolution of a dipole (2-point function): BK}

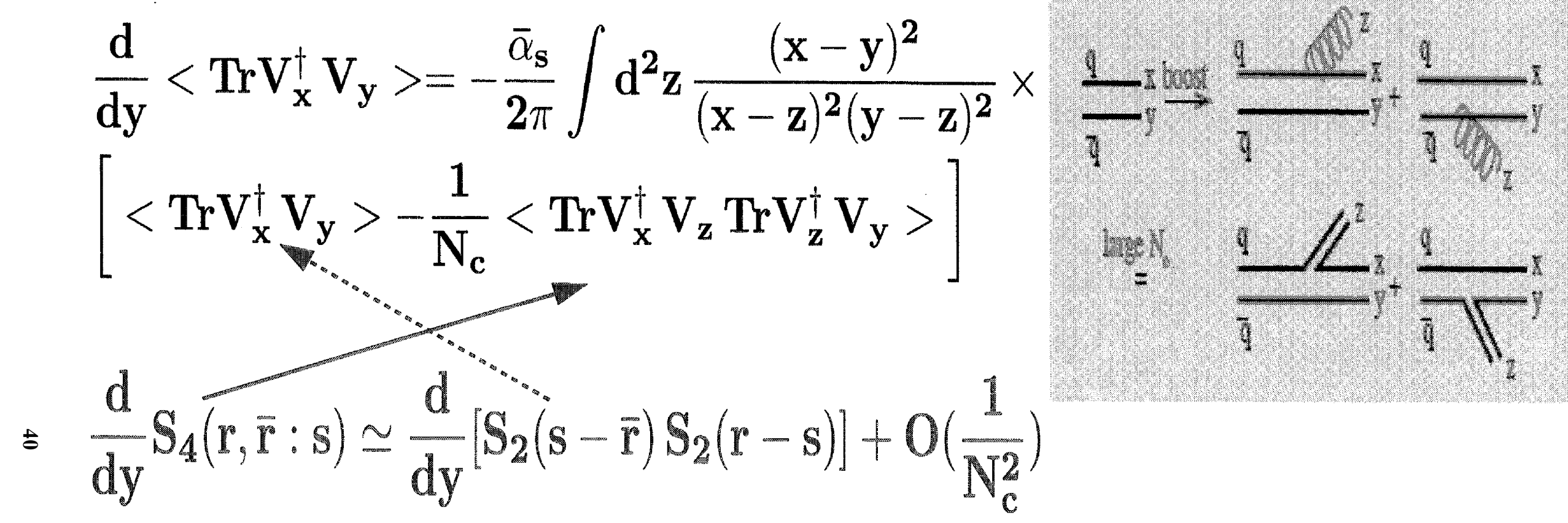

DIS F2,FL

DY in PA are sensitive

to dipoles only

Dijet production probes quadrupoles

A quadrupole is not the same as dipole $X$ dipole AD-JJM, 2011 


\section{Dilepton production: $\mathbf{k}_{\mathrm{t}}$ integrated}

$$
\frac{d \sigma^{d(p) A \rightarrow l^{+} l^{-} X}}{d^{2} b_{t} d M^{2} d x_{F}}=\frac{\alpha_{e m}^{2}}{6 \pi^{2}} \frac{1}{x_{q}+x_{g}} \int_{x_{q}}^{1} d z \int d r_{t}^{2} \frac{1-z}{z^{2}}
$$

F. Gelis \& JJM 02, JJM 04

$$
F_{2}^{d(p)}\left(x_{q} / z\right) \gamma\left(x_{g}, b_{t}, r_{t}\right)
$$

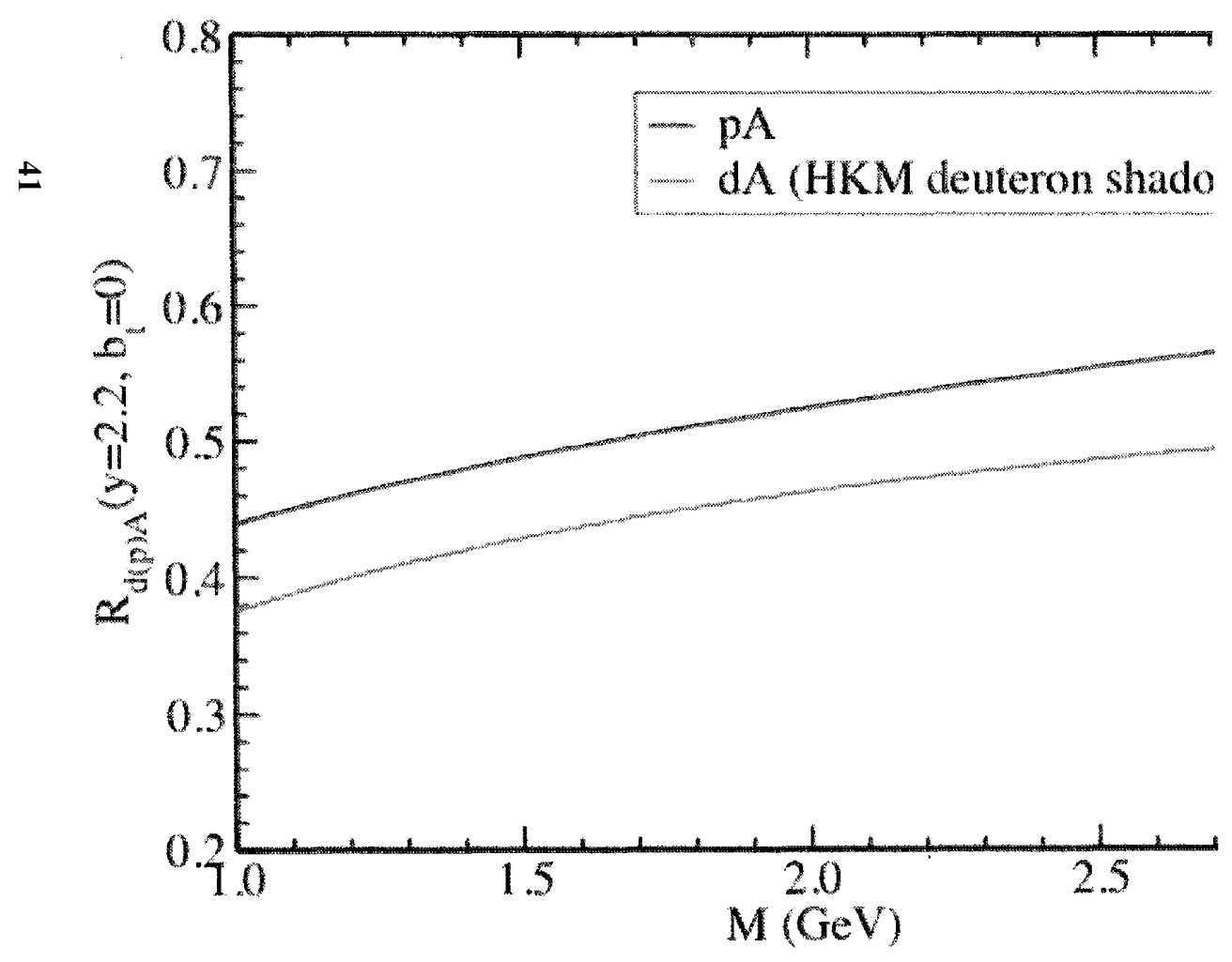

$$
\begin{gathered}
{\left[\left[1+(1-z)^{2}\right] K_{1}^{2}\left[\frac{\sqrt{1-z}}{z} M r_{t}\right]\right.} \\
\left.+2(1-z) K_{0}^{2}\left[\frac{\sqrt{1-z}}{z} M r_{t}\right]\right] \\
x_{F} \equiv \frac{M}{\sqrt{s}}\left[e^{y}-e^{-y}\right]
\end{gathered}
$$




\section{RHIC: $\mathbf{k}_{\mathrm{t}}=\mathbf{3} \mathrm{GeV}, \mathbf{y}=\mathbf{2}$}
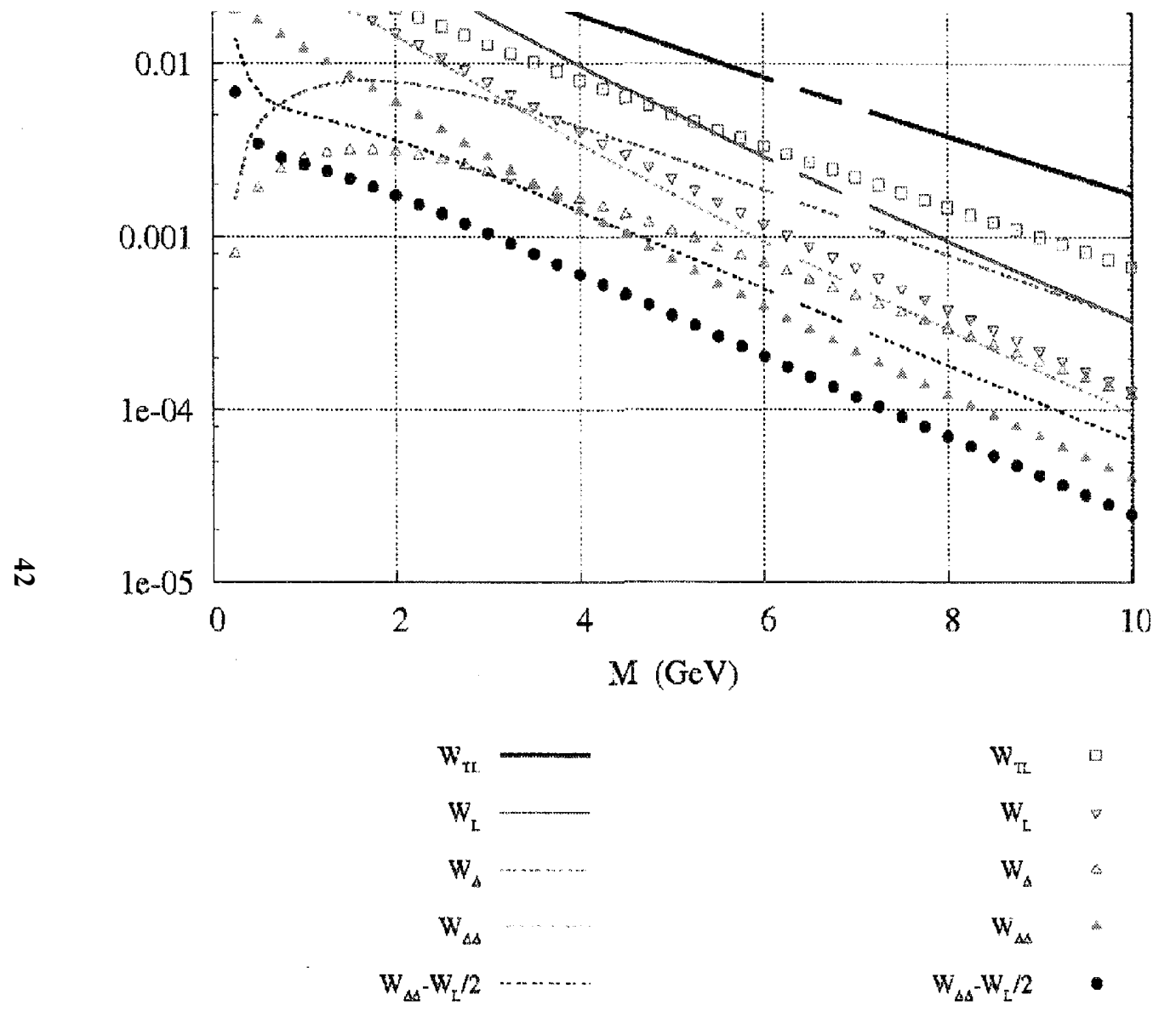
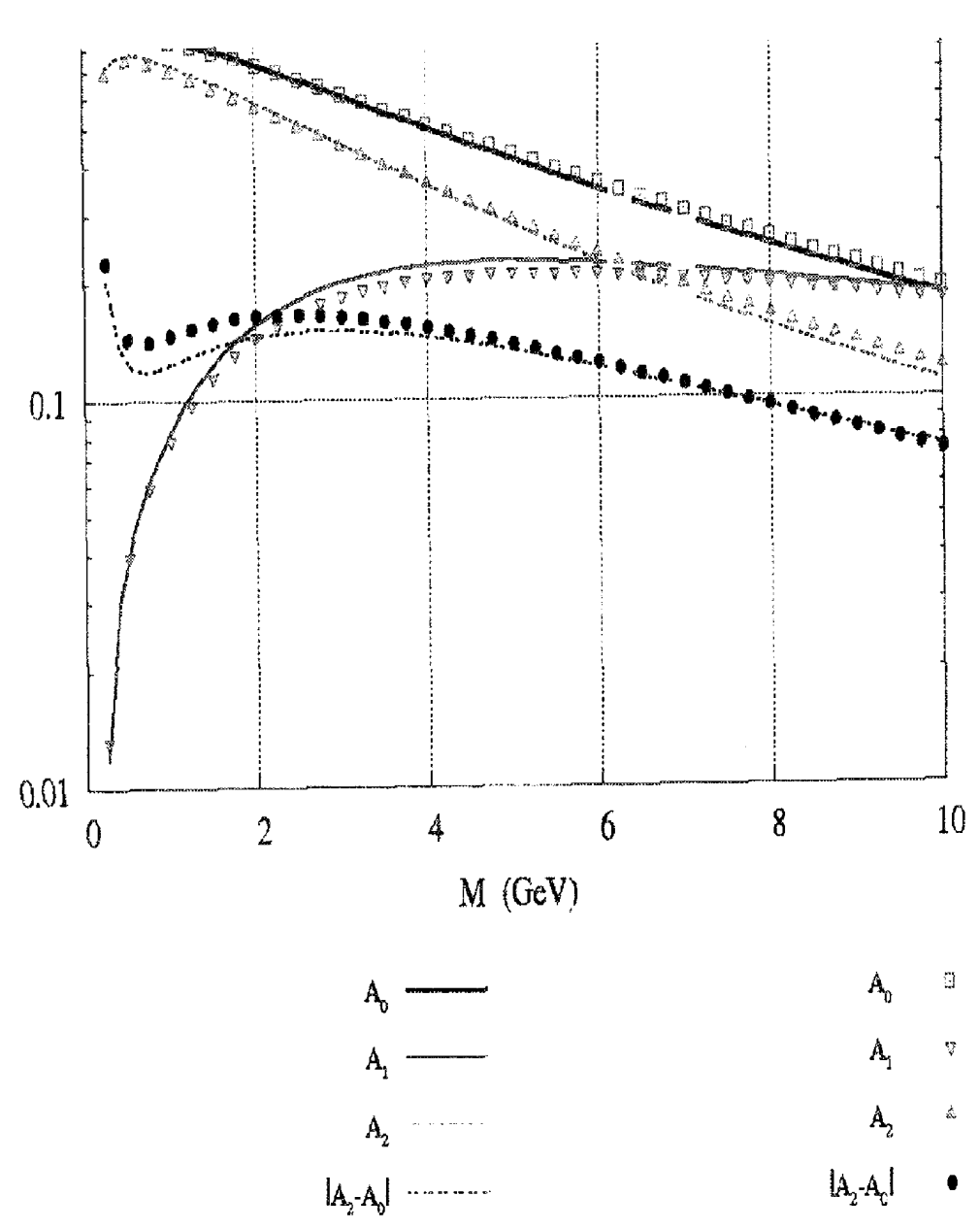

\section{Lines: (fixed coupling) BK}

Points: DHJ 


\title{
What can we learn with Drell-Yan in $\mathrm{p}(\mathrm{d})$ - Nucleus collisions
}

\author{
Feng Yuan \\ Lawrence Berkeley Lab/RBRC
}

* We argue that the forward Drell-Yan lepton pair production can be used to probe the nontrivial QCD dynamics associated with small-x physics. In particular, the saturation scale $Q_{s}{ }^{2}$ is order of $3-4 \mathrm{GeV}^{2}$, which is not too small compared to the hard scale: the invariant mass of the lepton pair. We estimate the nuclear suppression factor is less than 0.5 for small transverse momentum lepton pair production, which is unprecedented for Drell-Yan process. In this kinematics, the traditional DGLAP-based shadowing approach is not applicable any more. The Color-Glass-Condensate/Color-dipole approach is more suitable to describe these processes. We further argue that the single spin asymmetries in $\mathrm{pp}$ and $\mathrm{pA}$ collisions can provide more information on small-x physics, and $\sim$ may shed light on the underlying mechanism for the AN in various processes. 
Opportunities for Drell-Yan Physics at RHIC

In pi(d) Au Collisions

- Inclusive cross section

$\square$ Invariant mass not so large compared to the saturation scale

- Pt dependent observables

$\square$ Directly probe the unintegrated gluon distributions

$\square$ Correlation of DY-hadron

- Al's, Bowen's talks 


\section{How relevant is the saturation scale at RHIC}

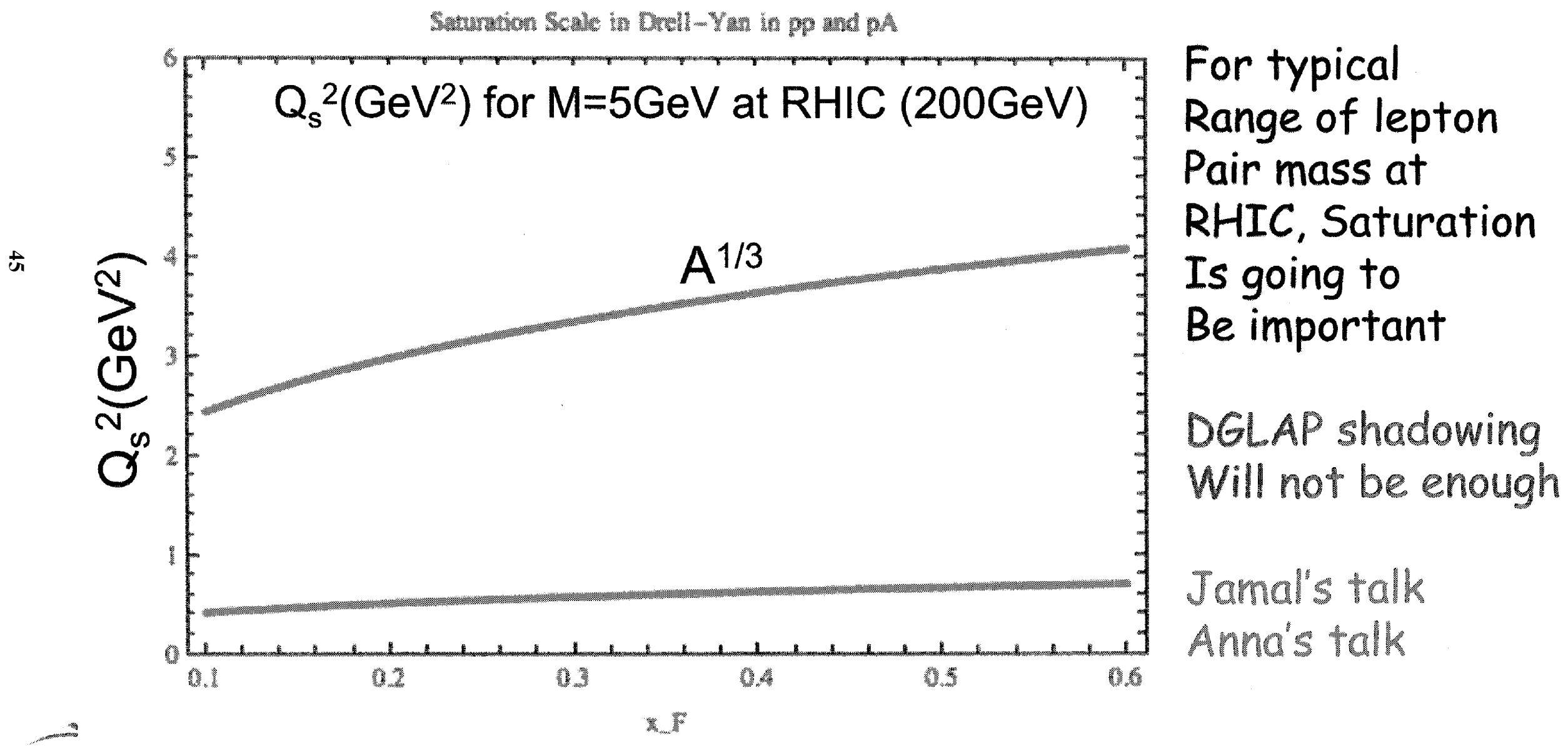




\section{Advantage of Low P+ Drell-Yan}

- Direct probe for the transverse momentum dependence of partons

. $\quad \square$ Saturation effects explicitly show up in the transverse momentum distribution

- Factorization can be argued for large $Q$

- Related to the TMD factorization

- Complementary study in SIDIS 
Pt dependence of the Nuclear suppression

Suppression Factor of Drell -Yan in pA

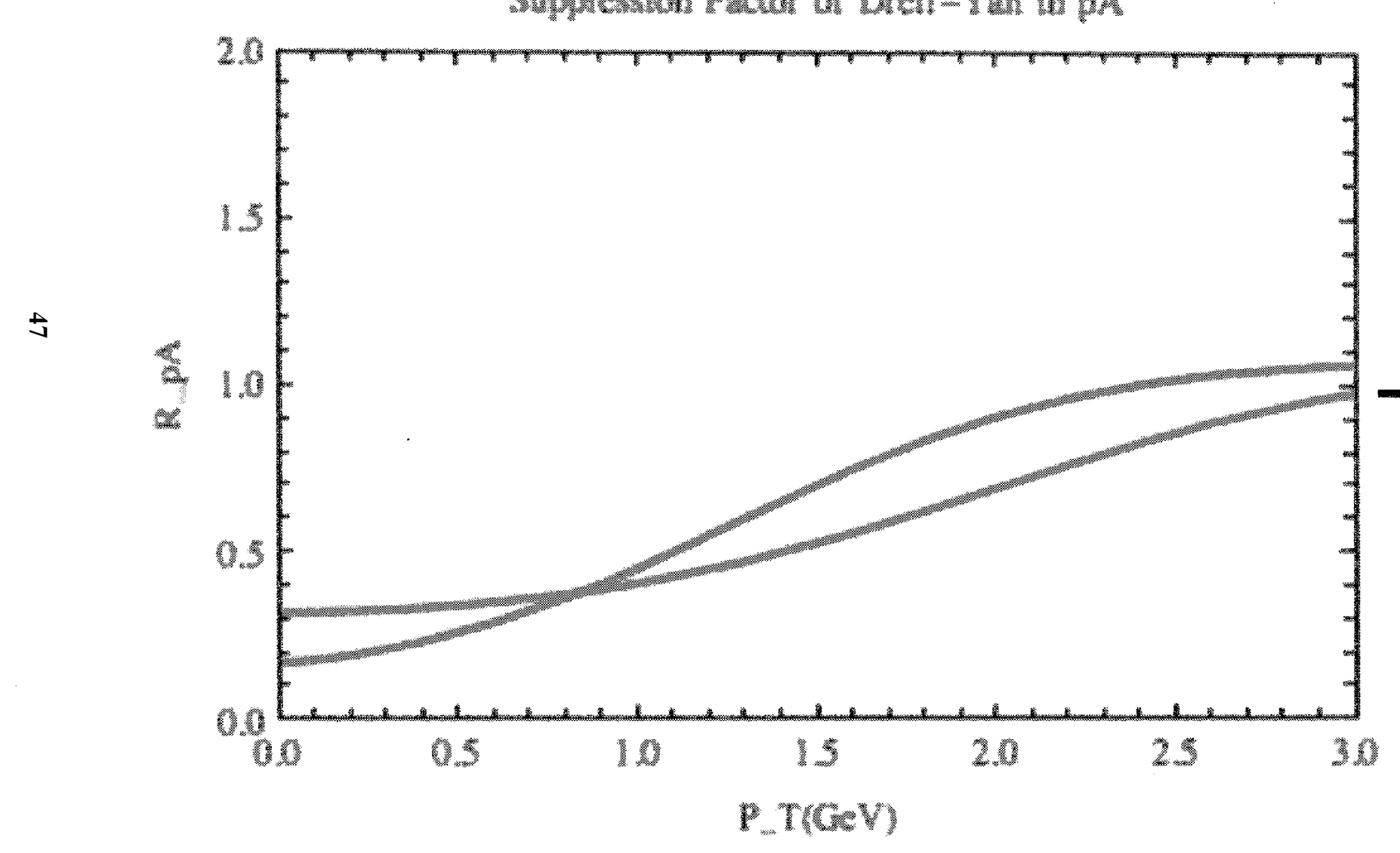

$$
R_{p A}=\frac{d \sigma^{p A \rightarrow \gamma^{*}}}{A d \sigma^{p p \rightarrow \gamma^{*}}}
$$

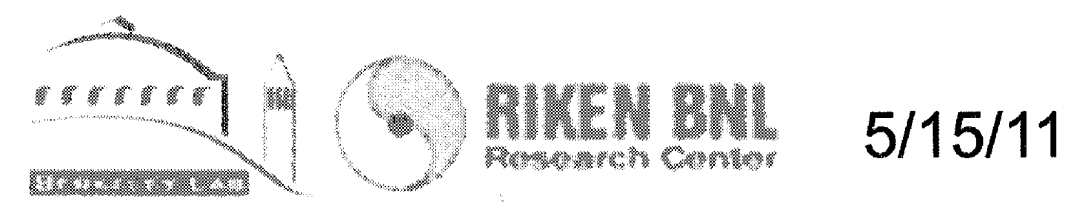

See also,

Guo, Qiu,Zhang, 00 


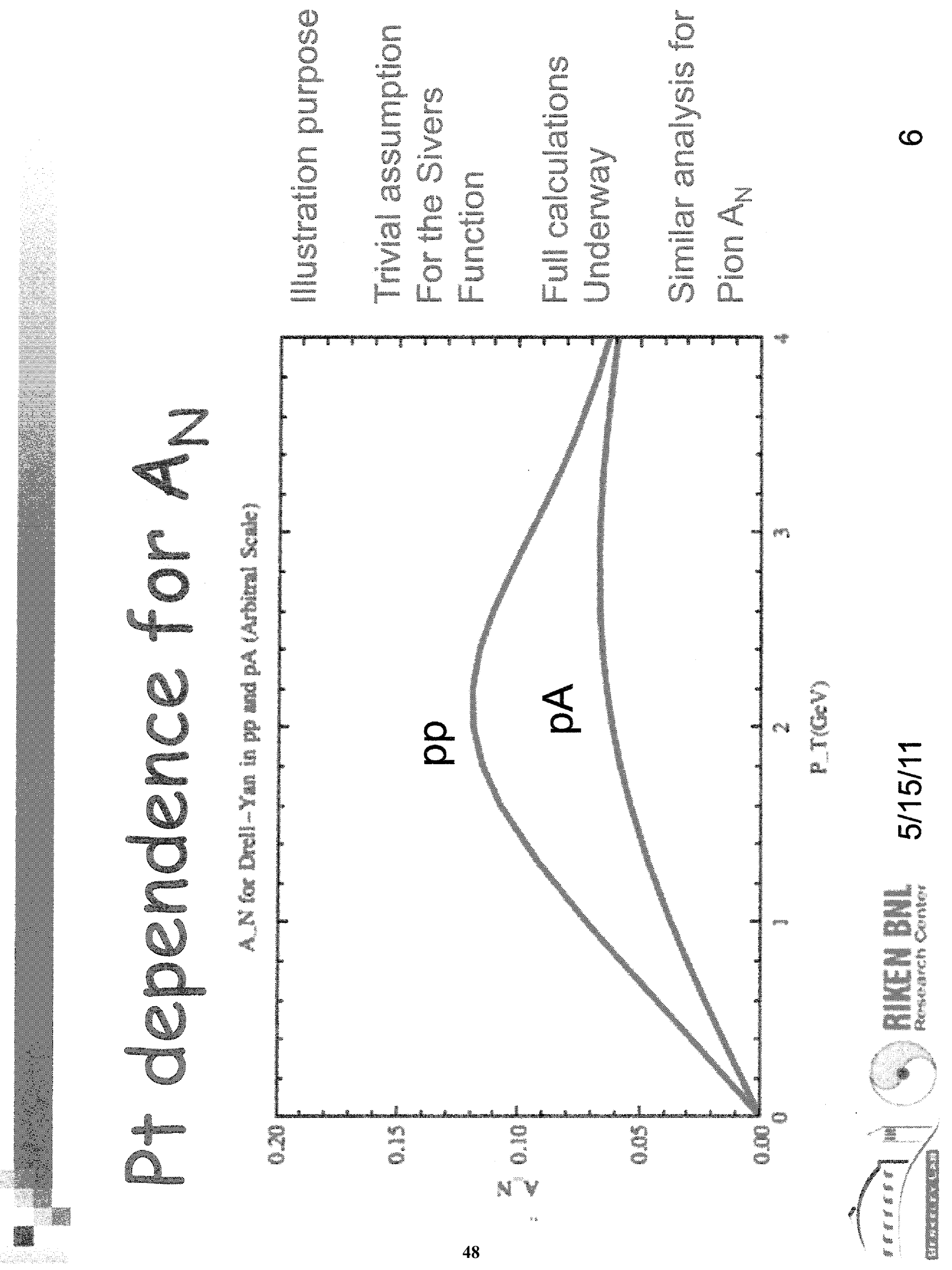




\section{TMD Universality}

\section{P.J. Mulders}

Nikhef and VU University, Amsterdam

The basic idea of PDFs is achieving a factorized description with soft and hard parts, soft parts being portable and hard parts being calculable. In the leading contributions at high energies, the PDFs can be interpreted as probabilities. Beyond the collinear treatment one considers not only the dependence on partonic momentum fractions $x$, but also the dependence on the transverse momentum $p_{T}$ of the partons. Experimentally, transverse momentum dependent functions (TMDs) provide a rich phenomenology of azimuthal asymmetries for produced hadrons or jet-jet asymmetries. Furthermore inclusion of transverse momentum dependence provides an explanation for single spin asymmetries.

An important issue is the universality of TMDs, which we study for some characteristic hard processes, where we focus on the pecularities coming from the color flow in the hard part. This color flow in the hard process gives rises to a variety of Wilson lines in the description of the cross section. These give rise to color entanglement, in particular in situations that the color flow is not just a simpie transfer of color from initial or final state.

We argue that these Wilson lines can be combined into the appropriate gauge links for TMD correlators in cases where only the transverse momentum of partons in a single (incoming) hadron is relevant (1parton un-integrated or 1PU processes). Such a situation occurs in single weighted cross sections, which consists of a sum of $1 \mathrm{PU}$ processes or if absence of any polarization makes all explicit transverse momentum effects vanish. For 1PU processes one finds TMDs with a complex gauge link structure depending on the color flow of the hard process. In the case of single weighted cross sections the results are the gluonic pole or Qiu-Sterman matrix elements appearing with calculable color factors.

I acknowledge discussions with Maarten Buffing (VU), Ted Rogers (VU) and Mert Aybat (VU and Nikhef). This research is part of the research programme of the Foundation for Fundamental Research of Matter and the National Organisation for Scientific Research (NOW). It is also part of the FP7 EU programme Hadron Physics (No 227431).

Summary of talk given at the Workshop on Opportunities for DY at RHIC, Brookhaven National Laboratory, May 11-13, 2001 


\section{Introduction}

- Isolating hard process (factorization)

- Study of quark and gluon structure of hadrons

- Account for hadronic physics to study hard process

- Beyond collinear approach

- Include mismatch of parton momentum $p$ and XP (fraction of

hadron momentum)

- TMDs with novel features

- Operator structure of TMDs

- Color gauge invariance as guiding principle

- Appearance of TMDs in hard processes

- Gauge links in 1-particle un-integrated (1PU) processes

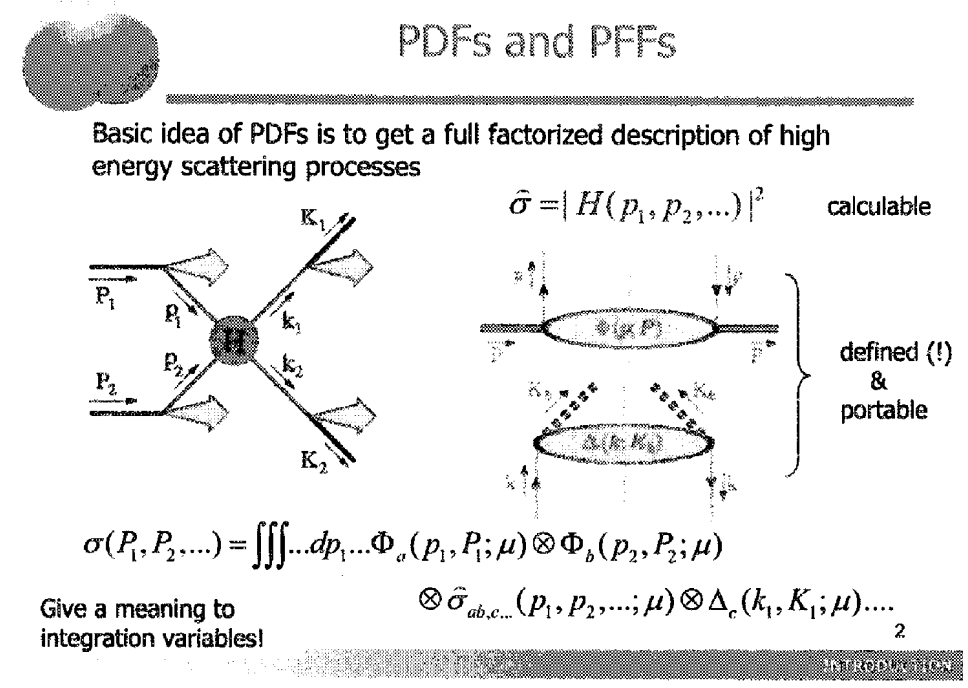

\section{Example: Drell-Yan process}

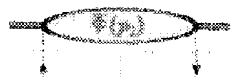

$$
\begin{aligned}
& \rightarrow \rightarrow m a m \cdot \quad \sum u\left(p_{1}, s\right) \bar{u}\left(p_{1}, s\right) \\
& \Rightarrow \quad \Rightarrow \Phi\left(p_{1}, P_{1}\right) \sim\left(p_{1}+m\right) f\left(p_{1}\right)
\end{aligned}
$$

- High energy limits number of soft matrix elements that contribute (twist expansion).

- Expand parton momenta (for DY take e.g. $n=P_{2} / p_{1} \cdot p_{2}$ )

$$
\begin{array}{rll}
p=x P^{\mu}+p_{7}^{\mu}+o n^{\mu} & x=p^{+}=p . n \sim 1 \\
\nearrow & \sim \mathrm{Q} \sim \mathrm{M} \sim \mathrm{M}^{2} / \mathrm{Q} & \sigma=p . P-x M^{2} \sim M^{2}
\end{array}
$$

- For meaningful separation of hard and soft, integrate over p.P and look at $\Phi\left(x, p_{T}\right)$. This shows that separation fails beyond 'twist 3 '.

$$
\text { 4.9.2. }
$$

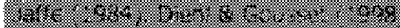

\section{Integrated quark correlators:} collinear and $7 \mathrm{MD}$

- Rather than considering general correlator $\Phi(p, P, \ldots)$, one integrates over p.P $=\mathrm{p}^{-}\left(\sim \mathrm{M}_{\mathrm{R}}{ }^{2}\right.$, which is of order $\left.\mathrm{M}^{2}\right)$

$$
\Phi_{i j}^{q}\left(x, p_{T} ; n\right)=\int \frac{d(\xi . P) d^{2} \xi_{T}}{(2 \pi)^{3}} e^{i p . \xi}\left\langle P\left|\psi_{i}(0) \psi_{i}(\xi)\right| P\right\rangle_{\xi, n=0} \quad \text { TMD }
$$

and/or $\mathrm{p}_{\mathrm{T}}$ (which is of order 1 ) lightfront

$$
\Phi_{i j}^{q}(x ; n)=\int \frac{d(\xi . P)}{(2 \pi)} e^{i p . \xi}\left\langle P\left|\bar{\psi}_{i}(0) \psi_{i}(\xi)\right| P\right\rangle_{\xi, n=\xi_{\eta}=0} \text { collinear }
$$

- The integration over $\mathrm{p}=\mathrm{p} . \mathrm{P}$ makes time-ordering automatic. This works for $\Phi(x)$ and $\Phi\left(x, p_{T}\right)$

- This allows the interpretation of soft (squared) matrix elements as forward antiquark-target amplitudes (untruncated!), which satisfy particular analyticity and support properties, etc. 


\section{Relevance of transverse momenta?}

- At high energies fractional parton momenta fixed by $p_{1} \approx x_{1} P_{1}+p_{1}$ kinematics (external momenta)

$p_{2} \approx x_{2} P_{2}+p_{2}$ DY $x_{1}=p_{1} \cdot n=\frac{p_{1} \cdot P_{2}}{P_{1} \cdot P_{2}}=\frac{q \cdot P_{2}}{P_{1} \cdot P_{2}}$

- Asso possible for transverse momenta of partons

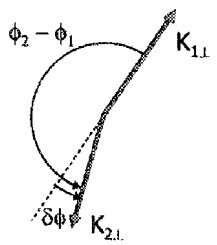

pp-scattering DY $q_{T}=q-x_{1} P_{1}-x_{2} P_{2}=p_{3 r}+p_{2 T}$

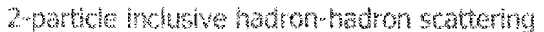

$$
\begin{aligned}
q_{T} & =z_{1}^{-1} K_{1}+z_{2}^{-1} K_{2}-x_{1} P_{1}-x_{2} P_{2} \\
& =p_{1 T}+p_{2 T}-k_{1 T}-k_{2 T}
\end{aligned}
$$

\section{Care is needed: we need more than one} hadron and knowledge of hard process(es)

\section{Opperunives of TMDS}

- TMD quark correlators (leading part, unpolarized) including T-odd part

$$
\Phi^{[ \pm] q}\left(x, p_{T}\right)=\left(f_{1}^{q}\left(x, p_{T}^{2}\right) \pm i h_{i}^{2, q}\left(x, p_{T}^{2}\right) \frac{\not p_{T}}{M}\right) \frac{\not p}{2}
$$

- Interpretation: quark momentum distribution $f_{1}{ }^{9}\left(x, p_{r}\right)$ and its

transverse spin polarization $h_{1} a\left(x, p_{T}\right)$ both in an unpolarized hadron

- The function $h_{1},{ }^{n}\left(x, p_{1}\right)$ is T-odd (momentum-spin correlations!)

- TMD gluon correlators (leading part, unpolarized)

$$
\Phi_{g}^{\mu v}\left(x, p_{T}\right)=\frac{1}{2 x}\left(-g_{T}^{\mu v} f_{1}^{*}\left(x, p_{T}^{2}\right)+\left(\frac{p_{T}^{\mu} p_{T}^{v}+\frac{1}{2} g_{T}^{\mu v}}{M^{2}}\right) h_{1}{ }^{2}\left(x, p_{T}^{2}\right)\right)
$$

- Interpretation: gluon momentum distribution $f_{1}^{9}\left(x, p_{1}\right)$ and its linear polarization $h_{1} g\left(x_{\ell}, p_{1}\right)$ in an unpolarized hadron (both are T-even)

\section{Twist expansion of (non-local) correlators}

Dimensional analysis to determine importance of matrix elements (just as for local operators)

maximize contractions with $n$ to get leading contributions

$$
\begin{aligned}
& \operatorname{dim}[\bar{\psi}(0) \not h \psi(\xi)]=2 \\
& \operatorname{dim}\left[F^{n \alpha x}(0) F^{n \beta}(\xi)\right]=2
\end{aligned}
$$

- 'Good' fermion fields and 'transverse' gauge fields

and in addition any number of $n \cdot A(\xi)=A^{n}(x)$ fields (dimension zero!) but in color gauge invariant combinations

$$
\begin{array}{ll}
\operatorname{dim} 0: & i \partial^{n} \rightarrow i D^{n}=i \partial^{n}+g A^{n} \\
\operatorname{dim} 1: & i \partial_{T}^{\alpha} \rightarrow i D_{T}^{\alpha}=i \partial_{T}^{\alpha}+g A_{T}^{\alpha}
\end{array}
$$

- Transverse momentum involves 'twist 3 '.

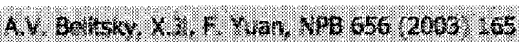

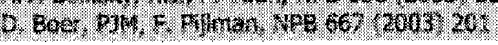

Gauge link results from leading gluons

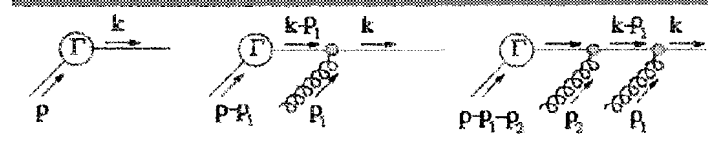

Expand gluon fields and reshuffle a bit:

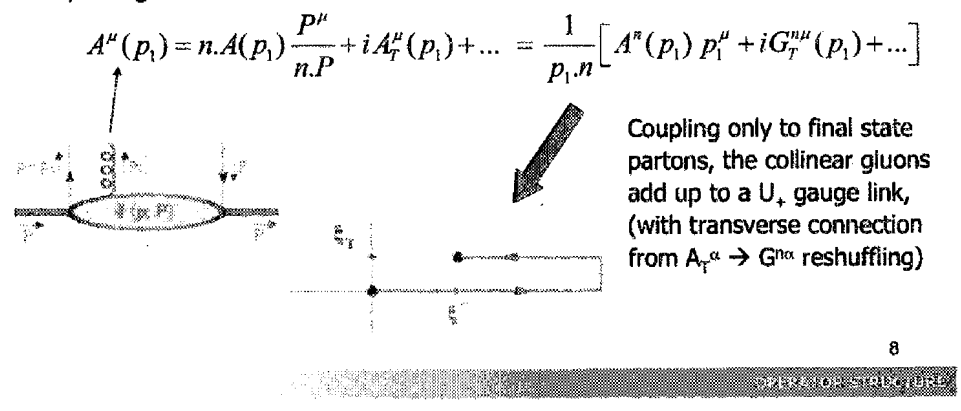


Gauge-invariant definition of TMDs: which gauge links?

\begin{tabular}{|c|}
\hline$\Phi_{i /}^{q[C]}\left(x, p_{T} ; n\right)=\int \frac{d(\xi \cdot P) d^{2} \xi_{T}}{(2 \pi)^{3}} e^{i p \cdot \xi}\left\langle P\left|\ddot{\psi}_{i}(0) U_{[0, \xi]}^{[C]} \psi_{i}(\xi)\right| P\right\rangle_{\xi, n: 0}$ \\
\hline$\Phi_{i}^{q}(x ; n)=\int \frac{d(\xi . P)}{(2 \pi)} e^{i f, \xi}\left\langle P\left|\bar{\psi}_{i}(0) U_{[0, \xi]}^{[n]} \not / \psi_{i}(\xi)\right| P\right\rangle_{\xi \xi n * \xi_{F}=0}$ \\
\hline
\end{tabular}

- Even simplest links for TMD correlators non-trivial:

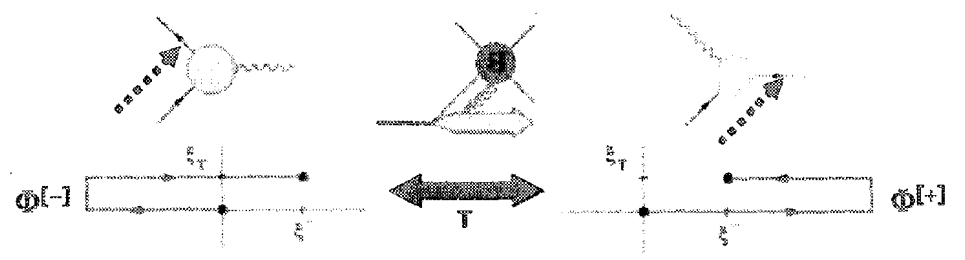

These merge into a 'simple' Wilson line in collinear $\left(p_{T}\right.$-integrated) case

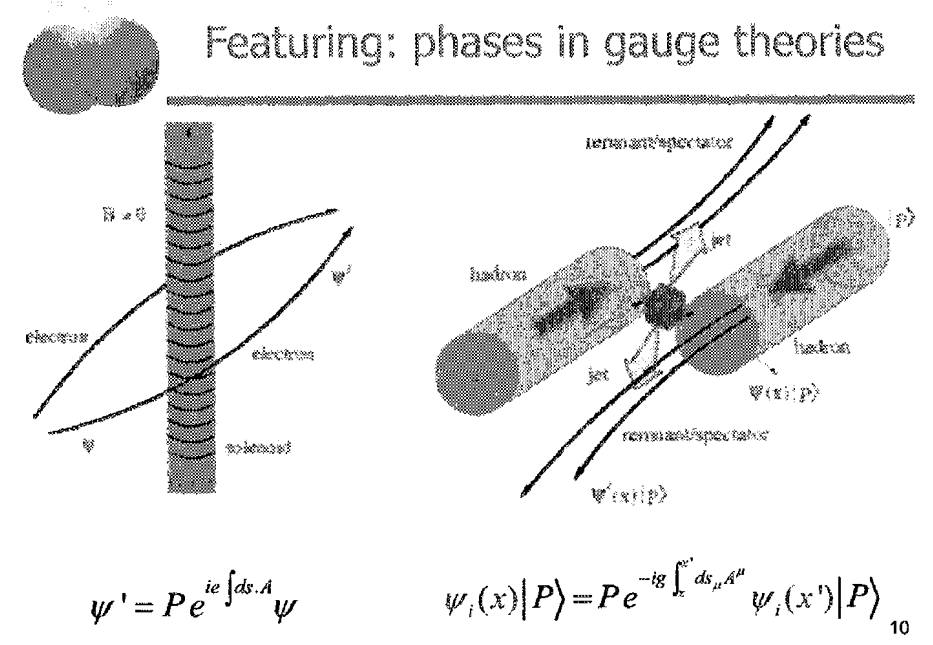

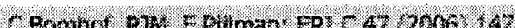

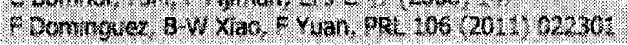
TMD correlators: gluons

$\Phi_{g}^{\alpha \beta[C, C]}\left(x, p_{T} ; n\right)=\int \frac{d(\xi . P) d^{2} \xi_{T}}{(2 \pi)^{3}} e^{i p, \xi}\left\langle P\left|U_{[\xi, 0]}^{[C]} F^{r, x \in}(0) U_{[0, \xi]}^{[C]} F^{n a p}(\xi)\right| P\right\rangle_{\xi, n=0}$

The most general TMD gluon correlator contains two links, which in general can have different paths.

- Note that standard field displacement involves $\mathrm{C}=\mathrm{C}^{\prime}$

$$
F^{\alpha \beta}(\xi) \rightarrow U_{[\eta, \xi]}^{[C]} F^{\alpha \beta}(\xi) U_{[\xi, \eta]}^{[C]}
$$

- Basic (simplest) gauge links for gluon TMD correlators:

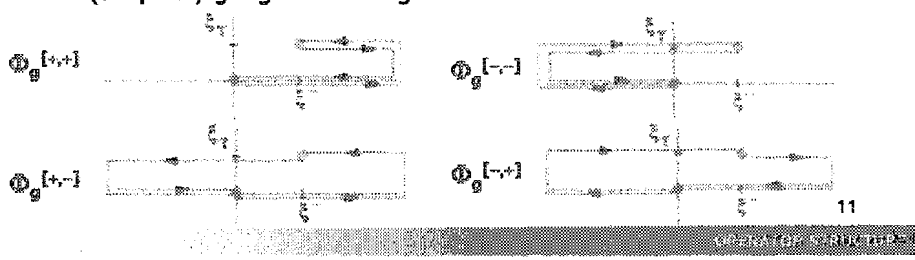

\section{Gauge invariance for DY}

$$
\bar{\psi}\left(\xi_{2}\right) \psi\left(0_{2}\right) \quad U_{\left\{0_{1}, \cdots \infty\right]} U_{\left[-\infty, \xi_{3}\right]} U_{\left[\xi_{2}, \cdots \infty\right]} U_{\left[-\infty, 0_{2}\right]}
$$$$
=W_{-\left[0, \xi_{1}\right]}^{[n]} W_{-\left[0_{2}, \xi_{2}\right]}^{[n]}=W_{-}^{[n]}\left[p_{1} W_{-}^{[n] t}\left[p_{2}\right]\right.
$$

$\left[-\infty, \xi_{1}\right](1$

- Mm Mm * i

$$
\frac{\left[\xi_{2},-\infty\right]}{\psi\left(\xi_{1}\right) \overline{\psi\left(y_{1}\right)}}
$$

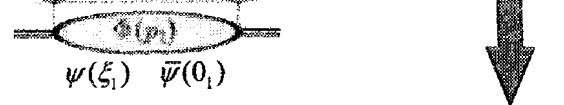

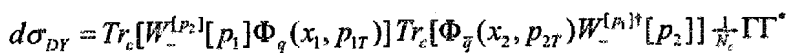

$$
\begin{aligned}
& =\Phi_{q}^{[-1}\left(x_{1}, p_{1 T}\right) \Phi_{\ddot{q}}^{[-\dagger]}\left(x_{2}, p_{2 T}\right) \hat{\sigma}_{\varphi \ddot{q} \rightarrow \gamma}
\end{aligned}
$$

Employing simple color fow possibilities, e.g. in $g g \rightarrow r$

J. Qiu, M. Schlegel, W. Vogelsang, ArXiv 1103.3861 (hep-ph) 

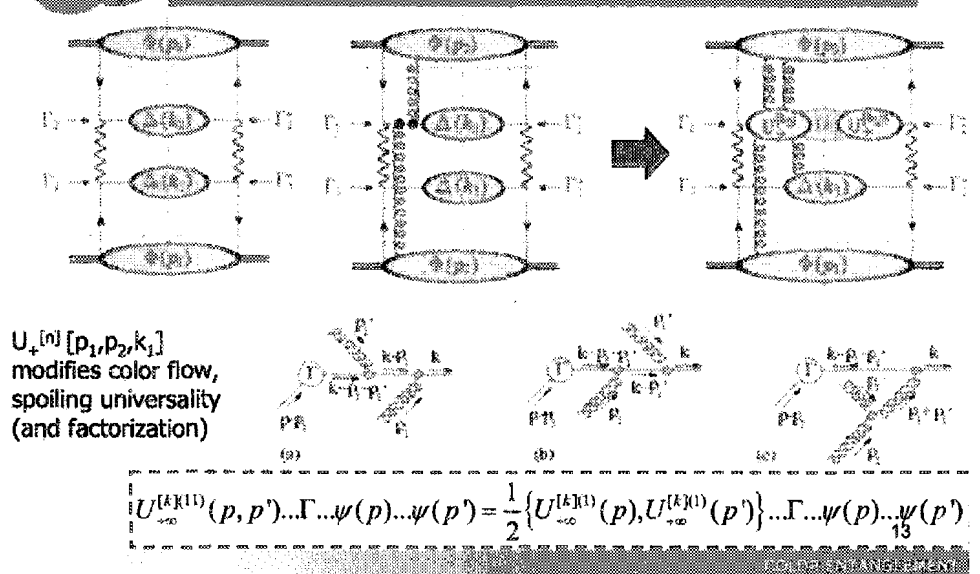

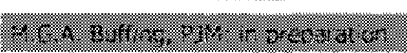

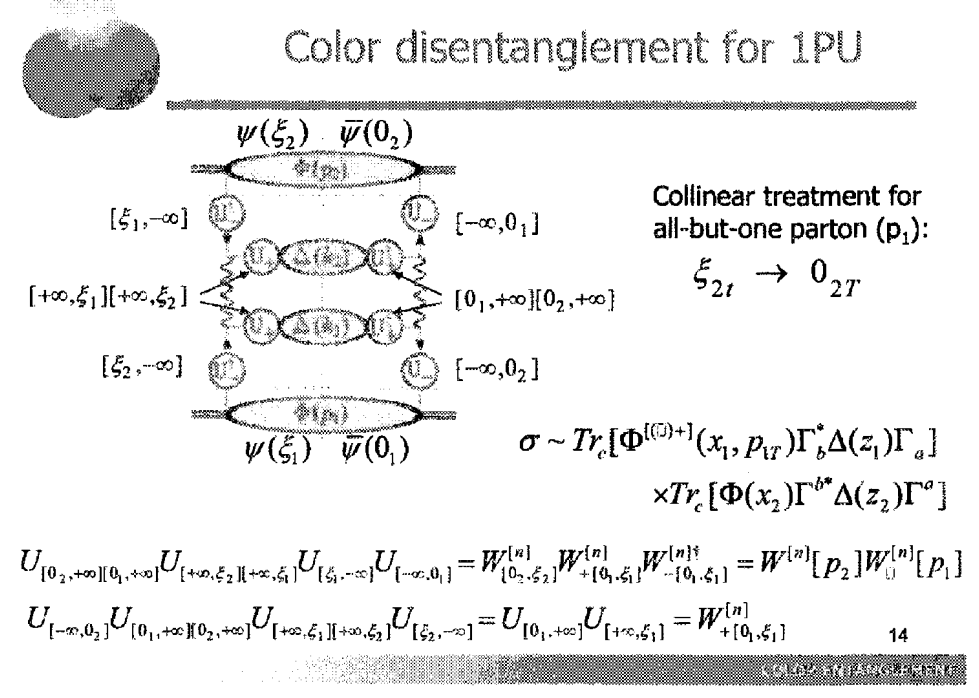
1 parton unintegrated

- Resummation of all phases spoils universality

- Transverse moments ( $\mathrm{p}_{\mathrm{T}}$-weighting) feels entanglement

Special situations for only one transverse momentum, as in single weighted asymmetries

$\int d^{2} q_{T} q_{T}^{\alpha} \ldots \int d^{2} p_{\mathrm{t} T} \int d^{2} p_{2 T} \ldots \delta^{2}\left(q_{T}-p_{1 T}-p_{2 T}\right)$ $=\int d^{2} p_{1 T} p_{1 T}^{\alpha} \int d^{2} p_{2 T} \ldots+\int d^{2} p_{1 T} \int d^{2} p_{2 T} p_{2 T}^{\alpha}$

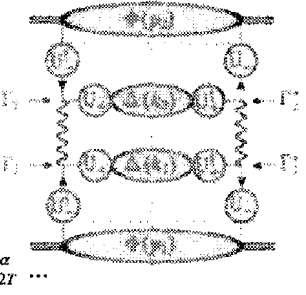

- But: it does produces 'complex' gauge links

- Applications of 1PU is looking for gluon $h_{1}^{19}$ (linear gluon polarization) using jet or heav quark production in ep scattering (e.g. EIC), (2011) 132001

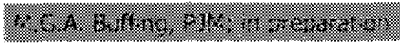
Full color disentanglement? NO!

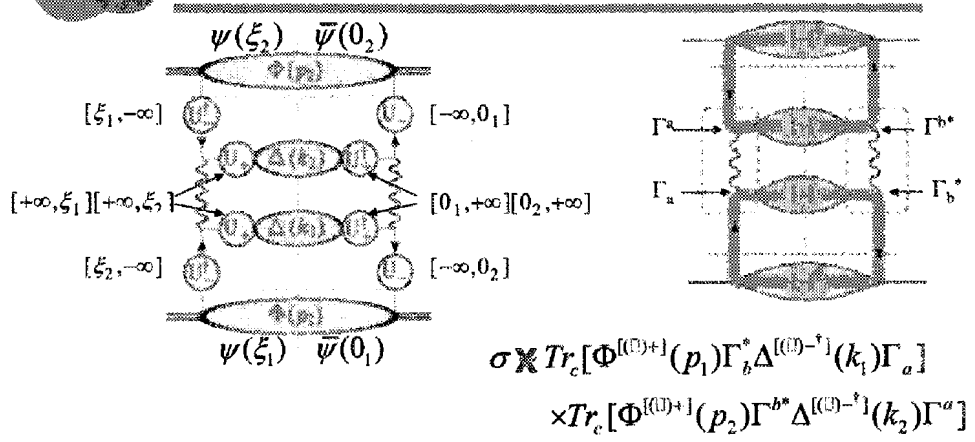

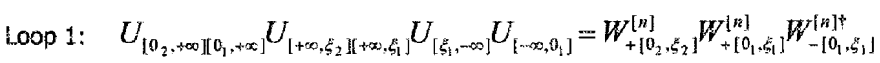
$=W_{+}^{[n]}\left[p_{2}\right] W_{1}^{[n]}\left[p_{1}\right]$ 


\section{Result for integrated cross section}

$\frac{d \sigma}{d^{2} p_{i T}} \sim \sum_{D, a b c} \Phi_{a}^{\left[C_{i}(D)\right]}\left(x_{1}, p_{i r}\right) \Phi_{b}\left(x_{2}\right) \hat{\sigma}_{a b \rightarrow c_{i}}^{[D]} \Delta_{c}\left(z_{1}\right) \ldots$

\section{Collinear cross section}

$$
\Phi^{\mathbb{X}}(x)=\int d^{2} p_{T} \Phi^{[c]}\left(x, p_{T}\right)
$$

Gauge link structure

$\sigma \sim \sum_{c d x:} \Phi_{a}\left(x_{1}\right) \Phi_{b}\left(x_{2}\right) \hat{\sigma}_{a b \rightarrow c . . .} \Delta_{c}\left(z_{1}\right) \ldots$

$$
\hat{\sigma}_{a b \rightarrow c \ldots}=\sum_{D} \hat{\sigma}_{a h \rightarrow c \ldots .}^{[D]} \quad \text { (partonic cross section) }
$$

Result for single weighted cross section

$$
\frac{d^{2}}{4}
$$$$
\frac{d \sigma}{d^{2} p_{i T}} \sim \sum_{D, a b c} \Phi_{a}^{\left[C_{i}(D)\right]}\left(x_{1}, p_{t r}\right) \Phi_{b}\left(x_{2}\right) \hat{\sigma}_{a b \rightarrow c \ldots .}^{[D]} \Lambda_{c}\left(z_{1}\right) \ldots
$$

$$
\left\langle p_{1 T}^{\alpha} \sigma\right\rangle \sim \sum_{D, a b c} \Phi_{\hat{\partial} a}^{\alpha[C(D)]}\left(x_{1}\right) \Phi_{b}\left(x_{2}\right) \hat{\sigma}_{a b \rightarrow c \ldots}^{[D]} \Delta_{c}\left(z_{1}\right) \ldots
$$

$$
\Phi_{\partial}^{\alpha[c]}(x)=\tilde{\Phi}_{\partial}^{\alpha[\$]}(x)+C_{G}^{[U / C]]} \pi \Phi_{G}^{\alpha[Q]}(x, x)
$$

$\left\langle p_{1 T}^{\alpha} \sigma\right\rangle \sim \sum \tilde{\Phi}_{a}^{\alpha}\left(x_{1}\right) \Phi_{b}\left(x_{2}\right) \hat{\sigma}_{a b \rightarrow c \ldots} \Delta_{c}\left(z_{1}\right) \ldots \quad \begin{aligned} & \Phi_{\mathrm{G}}(\mathrm{x}, \mathrm{x}) \text { is gluonic pole } \\ & \left(\mathrm{x}_{\mathrm{s}}=0\right) \text { matrix element }\end{aligned}$

T-odd part

$$
+\sum_{a b c} \pi \Phi_{G a}^{\alpha}\left(x_{1}, x_{1}\right) \Phi_{b}\left(x_{2}\right) \hat{\sigma}_{[a] h \rightarrow c,} \Delta_{c}\left(z_{1}\right) \ldots
$$

$$
\hat{\sigma}_{[a] b \rightarrow c \ldots}=\sum_{D} C_{G}^{[r(C(D))]} \hat{\sigma}_{a b \rightarrow c \ldots}^{[D]} \quad \begin{aligned}
& \text { (gluonic pole } \\
& \text { cross section) }
\end{aligned}
$$

\section{Higher $p_{\text {T }}$ moments}

- Higher transverse moments

$$
\Phi^{[N] \alpha_{i} \ldots \alpha_{N}}(x)=\int d^{2} p_{T}\left(p_{T}^{\alpha_{1}} \ldots p_{T}^{\alpha_{1}}-\text { traces }\right) \Phi\left(x, p_{T}\right)
$$

- involve yet more functions

$$
\tilde{\Phi}_{\partial i}^{\alpha \beta}(x), \tilde{\Phi}_{\tilde{\nu} G}^{\alpha \beta}(x, x), \Phi_{G G}^{\alpha \beta}(x, x, x)
$$

- Important application: there are no complications for fragmentation, since the 'extra' functions $\Delta_{G,} \Delta_{G G}$... vanish. using the link to 'amplitudes';

k. Gamberg, A. Mukherjee, PIM, PRD 83 (2011) 071503 (R)

- In general, by looking at higher transverse moments at tree-level, one concludes that transverse momentum effects from different initial state hadrons cannot simply factorize.

\section{Conclusions}

- Color gauge invariance produces a jungle of Wilson lines attached to all parton legs, although the gauge connections themselves have a nicely symmetrized form

- Easy cases are collinear and 1-parton un-integrated (1PU) processes, with in the latter case for the TMD a complex gauge link depending on the color flow in the tree-level hard process

- Example of 1PU processes are the terms in the sum of contributions to single weighted cross sections

- Single weighted cross sections involve T-even 'normal weighting' and T-odd gluonic pole matrix elements (SSA's)

- Gluonic pole matrix elements in fragmentation correlators vanish, thus treatment of fragmentation TMDs is universal (physical picture observation of jet direction)

- Furthermore, there is the issue of factorization! (talk Ted Rogers) 


\section{Gauge Links and TMD-Factorization}

\section{Ted Rogers}

\section{Vrije Universiteit Amsterdam}

I will provide a status overview of transverse momentum dependent factorization theorems, with an emphasis on evolution, universality/nonuniversality, and the issue of factorization breaking. I will start by reviewing the basic concepts of gauge links and the complications that arise when attempting to define parton correlation functions. I will also describe recent efforts to combine existing implementations of the Collins-Soper-Sterman evolution formalism with fixed scale fits of TMDs. The result is a set of TMD fits in transverse momentum space that include evolution. Emphasis will be placed on the relationship with more standard generalized parton model concepts. I will conclude with a discussion of our future plans to extend TMD phenomenology with evolution.

BNL Workshop on Drell-Yan Physics, May 11, 2011 


\section{Generalized TMD-factorization breaking:}

- Gluons have color.

Actual color structure

Color Entanglement

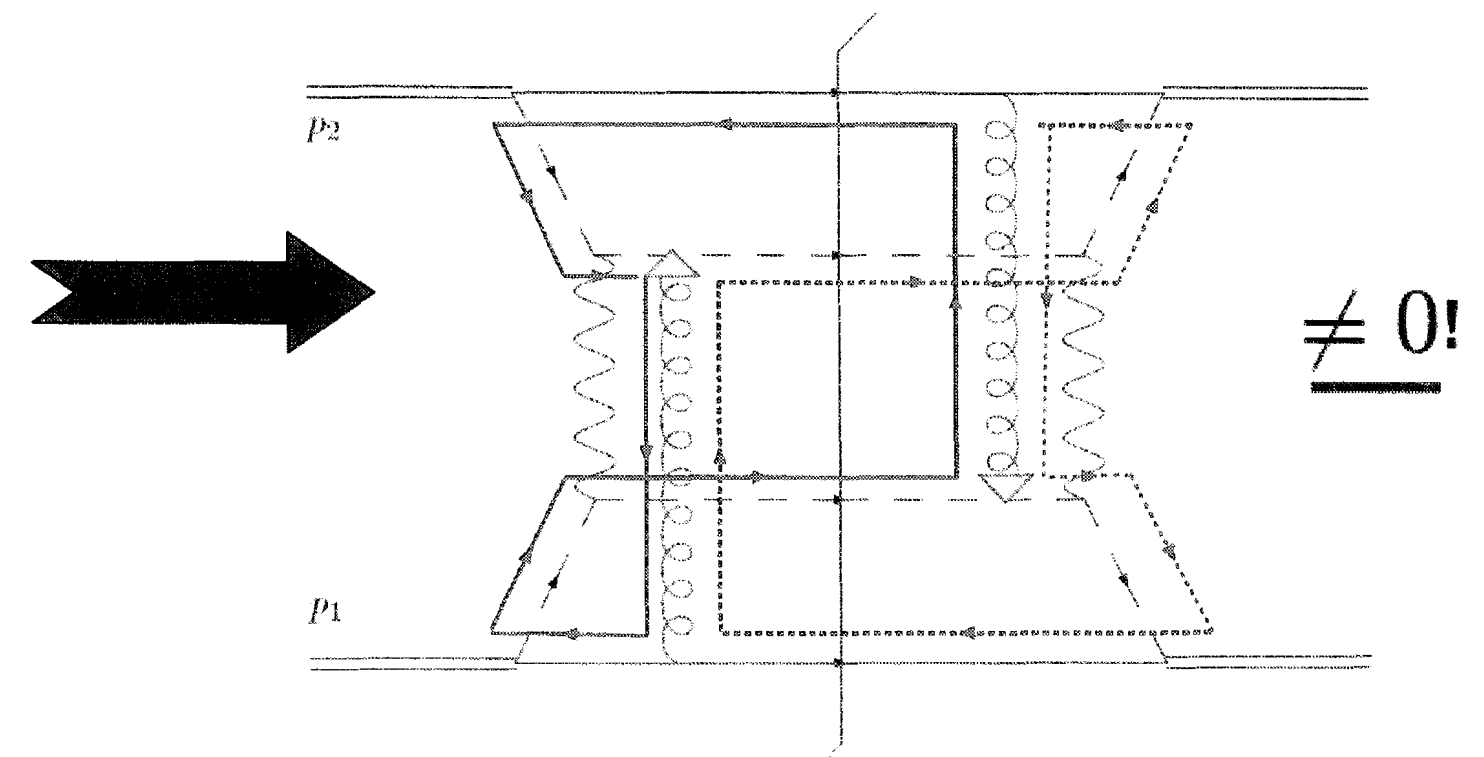

- "generalized" factorization formula:

(TCR, Mulders (2010))

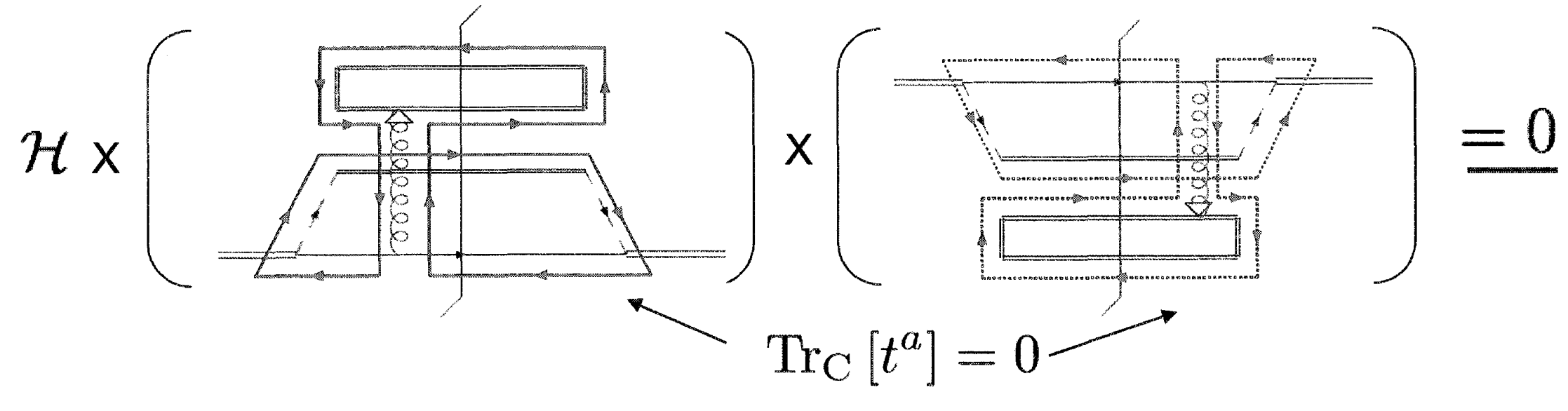




\section{TMD-Factorization:}

- Complications with defining TMDs:

- Divergences.

- Wilson lines / gauge links.

- Universality vs. non-universality.

- Definitions dictated by requirements for factorization!

-Processes:

- Semi-Inclusjive deep inelastic scattering.

- Drell-Yan.

- $\mathrm{e}^{+} / \mathrm{e}^{-}$annihilation.

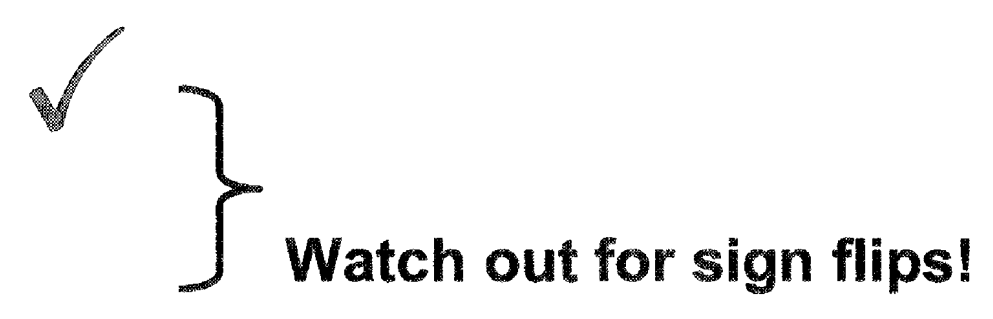

$=p+p=n_{1} h_{2}+x$

-Implementation and TMD phenomenology.

- Use existing fixed-scale fits / no evolution.

- Use existing "old fashion" implementation of Collins-Soper-Sterman formalism.

- Full TMD formalism, including evolution.

(New Collins Definitions) 


\section{TMD PDF, Complete Definition:}

$F_{f / P}\left(x, b ; \mu ; \zeta_{F}\right)=$

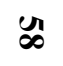

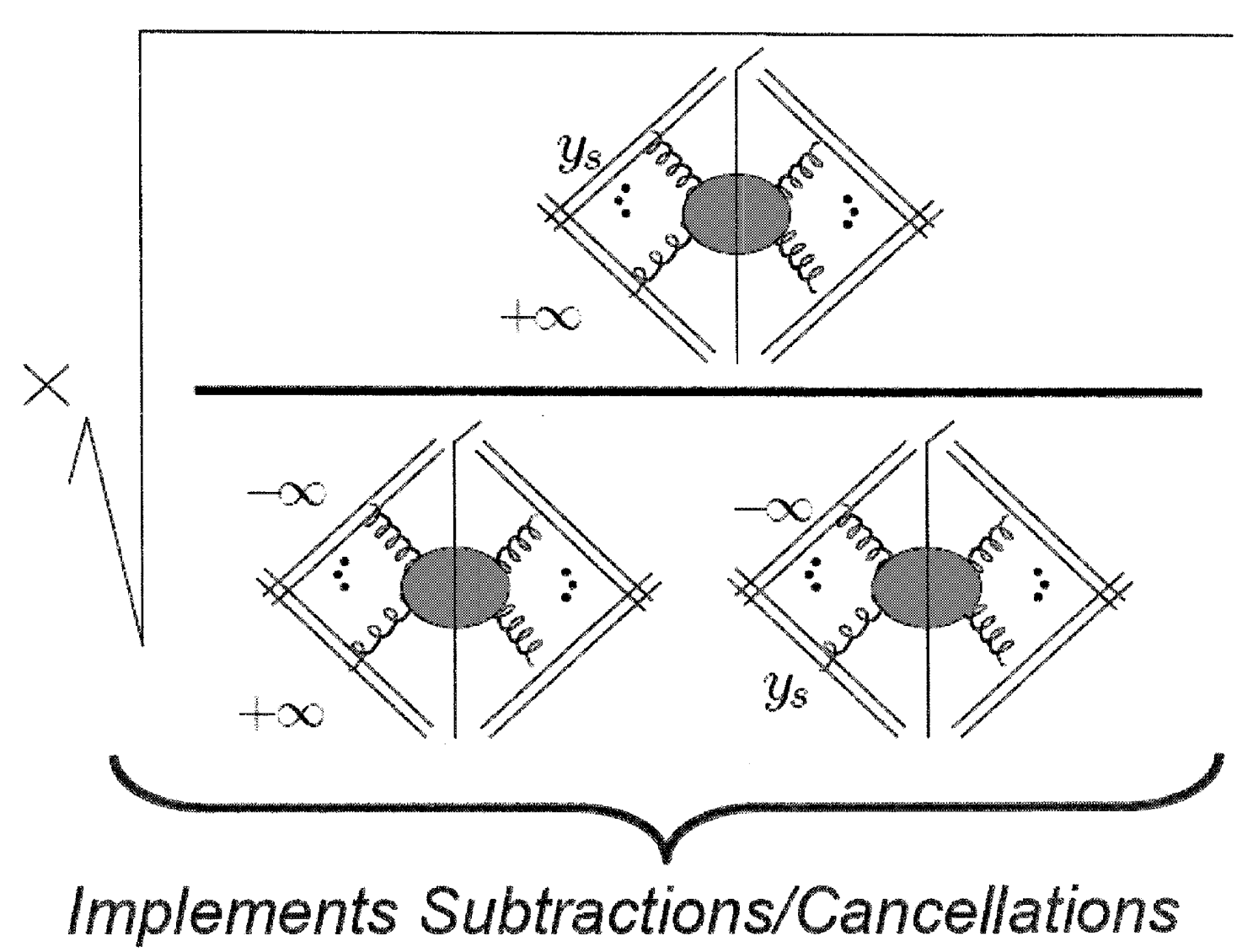

From Foundations of Perturbative QCD, J.C. Collins, (See also, Collins, TMD 2010 Trento Workshop) 


\section{Current Strategy:}

- Use evolution to combine existing fits into unified/global fits that include evolution.

(S.M. Aybat, TCR (2011))

- PDFs:

- Start with DY:

(Landry et al, (2003); Konychev, Nadolsky (2006)) (BLNY)

- Modify to match to SIDIS:

(Schweitzer, Teckentrup, Metz (2010)) (STM)

- Can supply explicit, evolved TMD PDF fit. 


\section{Evolving TMD PDFs}

Up Quark TMD PDF, $x=.09$

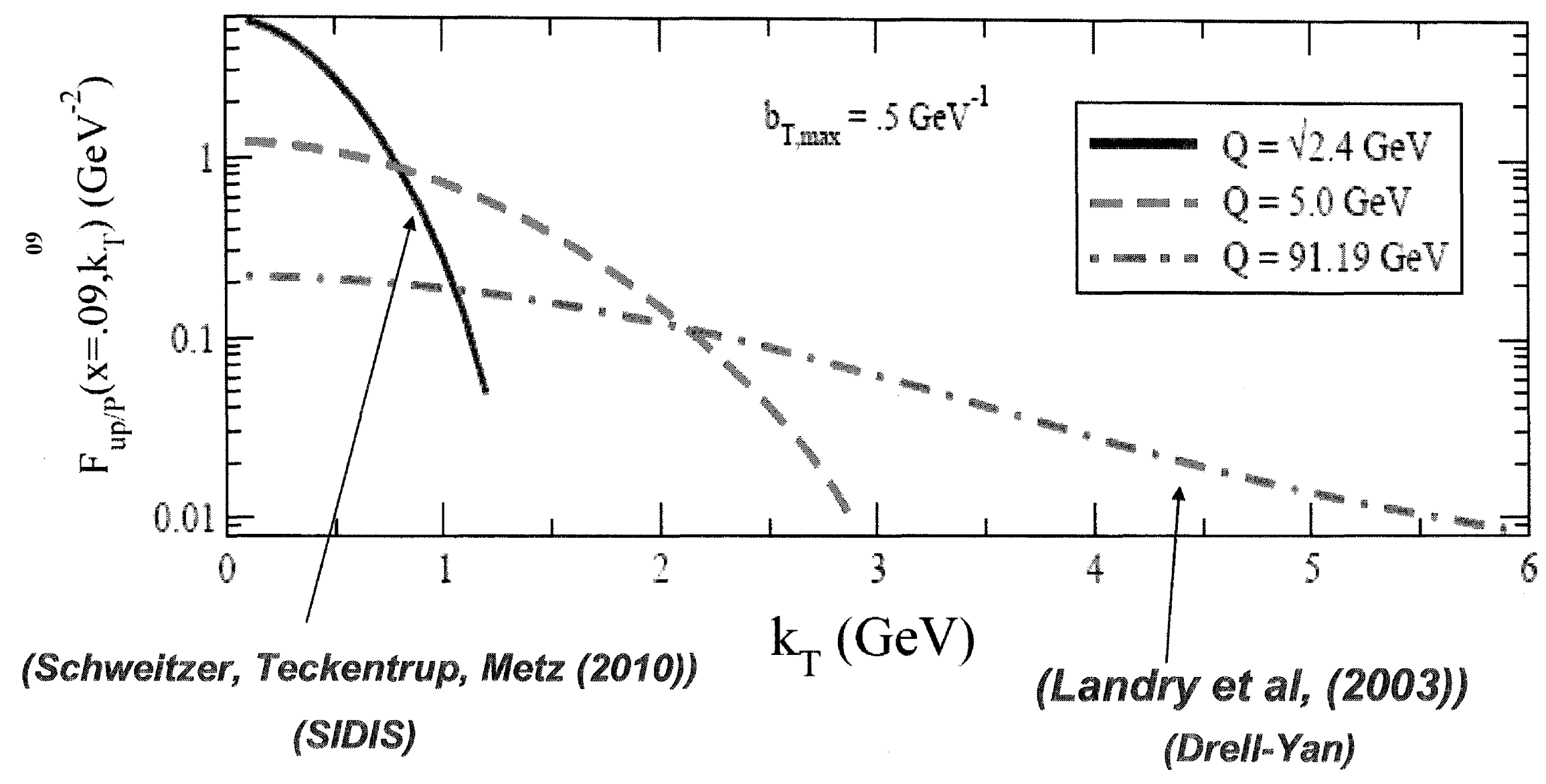




\section{Opportunities with Drell-Yan \\ Argonne \\ Scattering at Fermilab}

Paul E. Reimer

Physics Division

Argonne National Laboratory

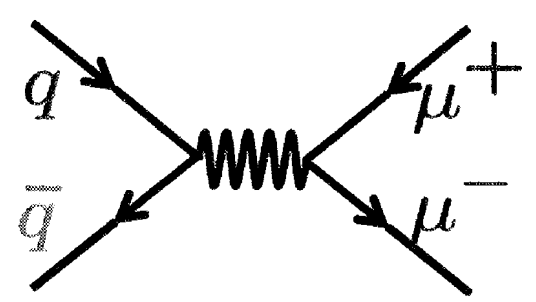

1. The Drell-Yan Process-A Laboratory for Quark Studies

2. Fermilab E-906/SeaQuest Physics Program

- Sea quark in the proton

- Sea quarks in the nucleus

- Angular distributions

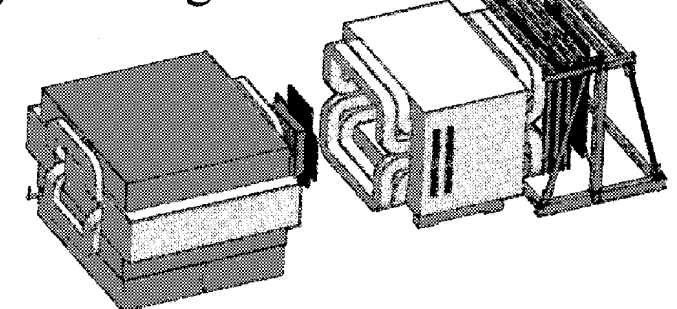

3. What can the future hold? Polarized targets or beams?

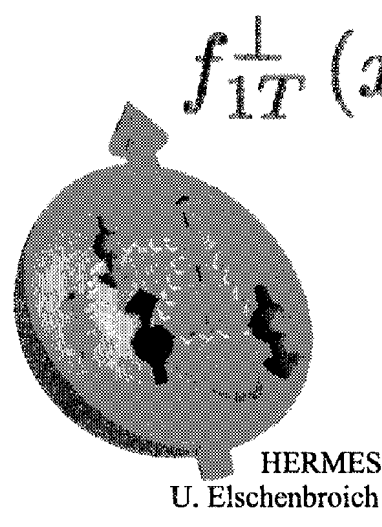

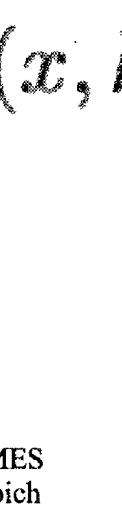

$$
\begin{gathered}
\left.\right|_{\text {DIS }}=-\left.f \frac{1}{1 T}\left(x, k_{T}\right)\right|_{\text {DY }} \\
\text { With help from Chiranjib Dutta, } \\
\text { Wolfgang Lorenzon, U. Michigan } \\
\text { and Yuji Goto, RIKEN }
\end{gathered}
$$

This work is supported in part by the U.S. Department of Energy, Office of Nuclear Physics, under Contract No. DE-AC02-06CH11357.
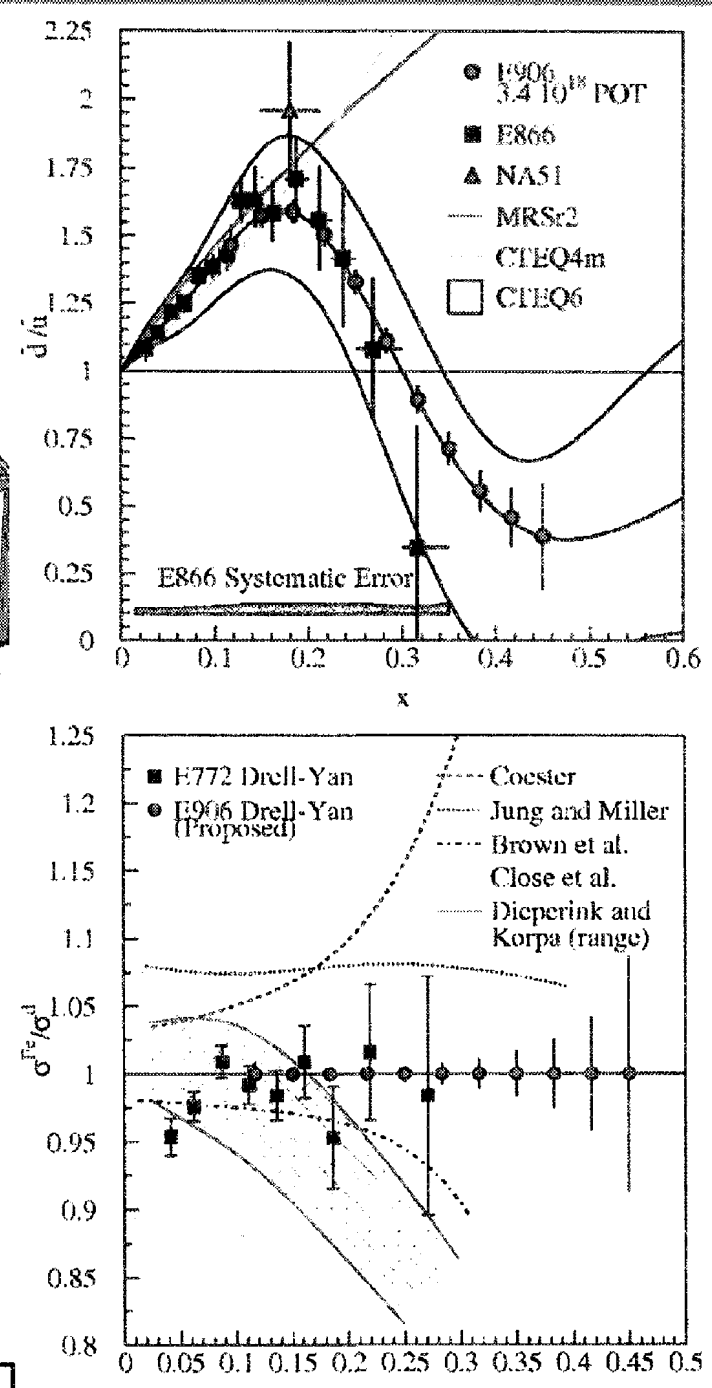

(2) ENERGY 


\section{Extracting d-bar/-ubar From Drell-Yan Scattering}
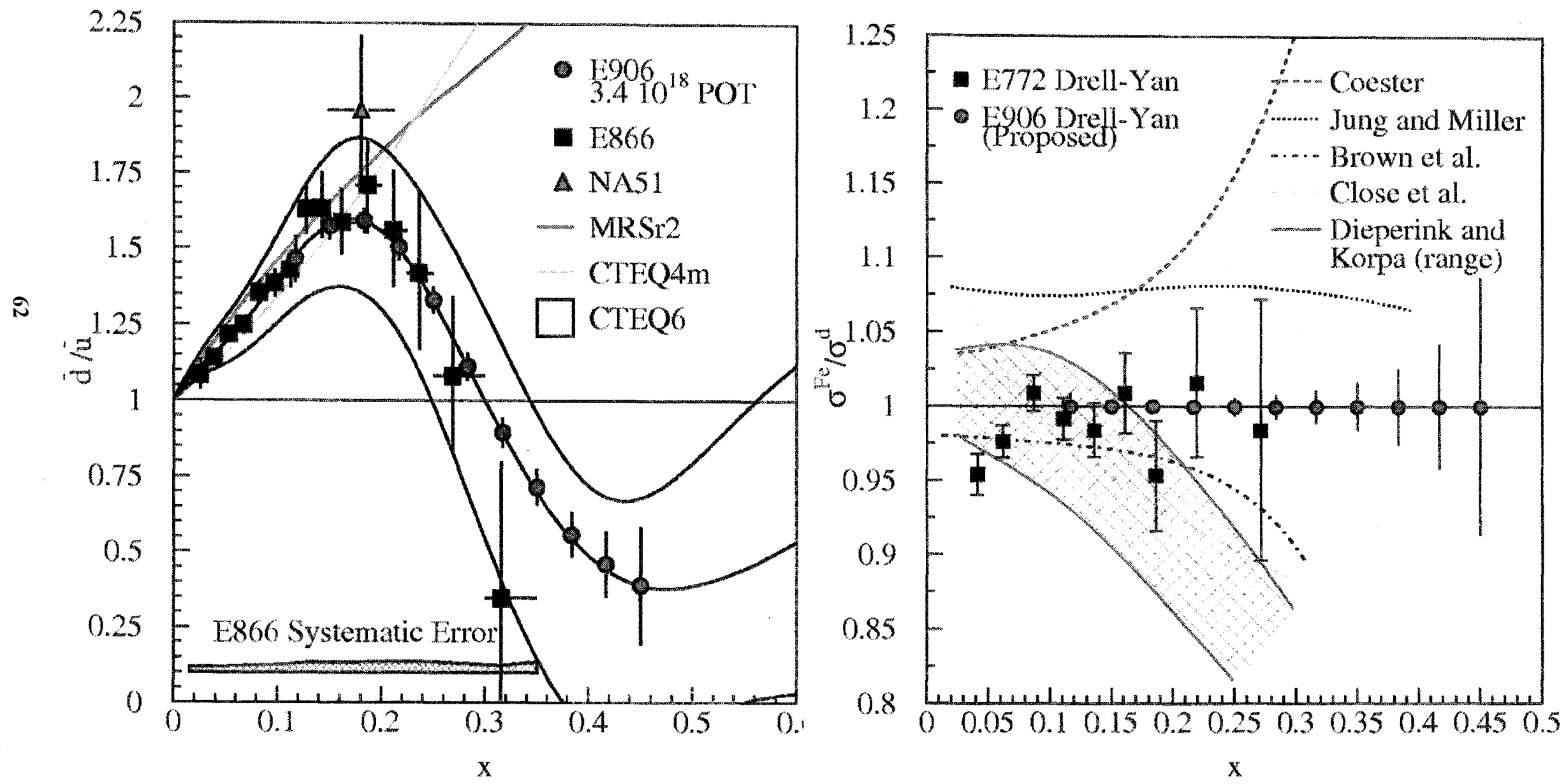


\section{Other Physics from E-906/SeaQuest}

- Absolute High- $\mathrm{x}_{\mathrm{Bj}}$ Parton Distributions

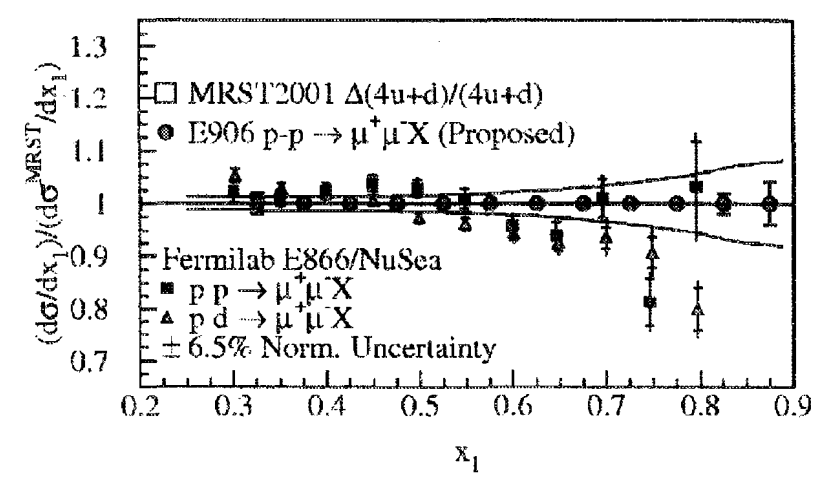

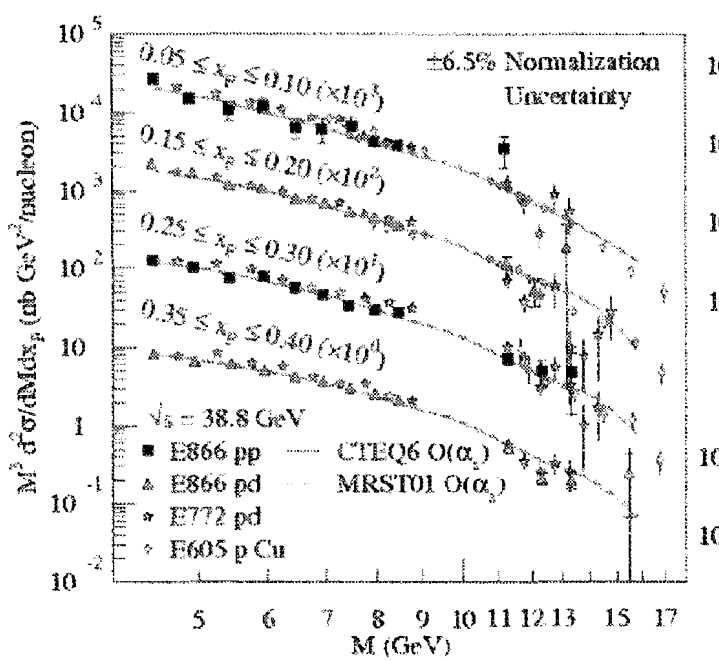

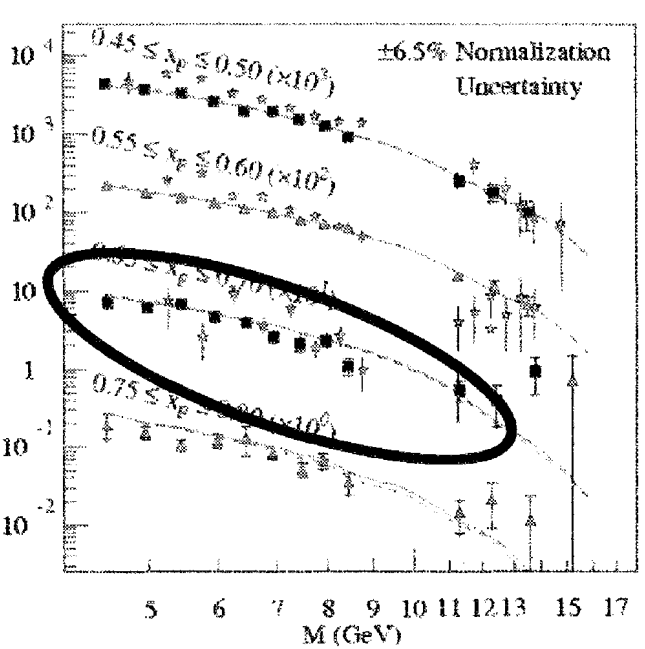

Partonic Energy Loss in Cold Nuclear Matter

Parton Loses Energy

in Nuclear Medium
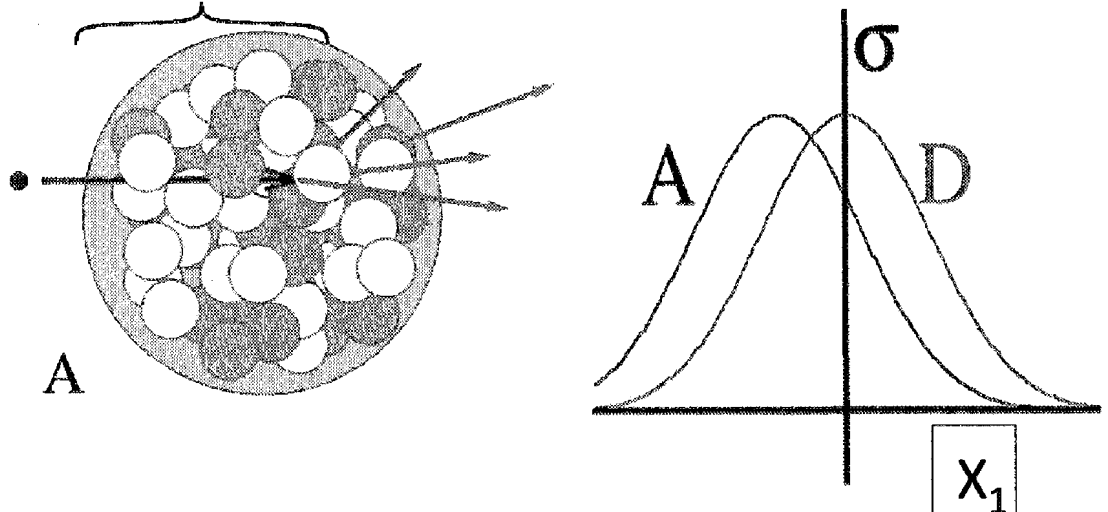

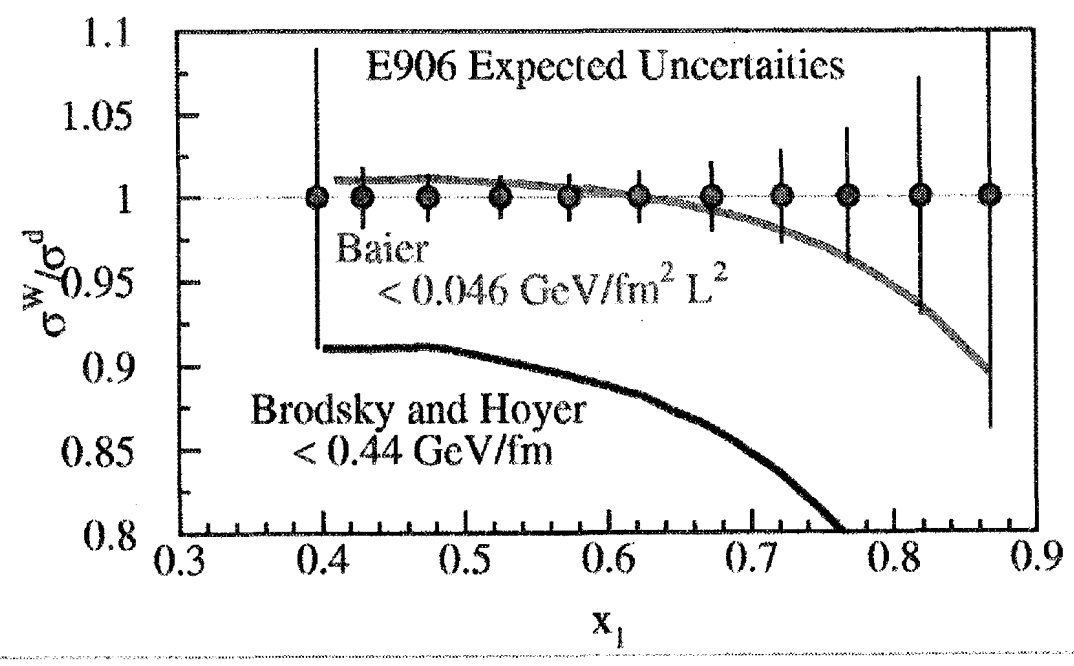




\section{E-906/SeaQuest timeline and plans}

- Fermilab PAC approved the experiment in 2001 - but experiment was not scheduled due to concerns about "proton economics"

- Fermilab Stage-II Approval granted on 24 December 2008

- Expected first beam in late June 2011

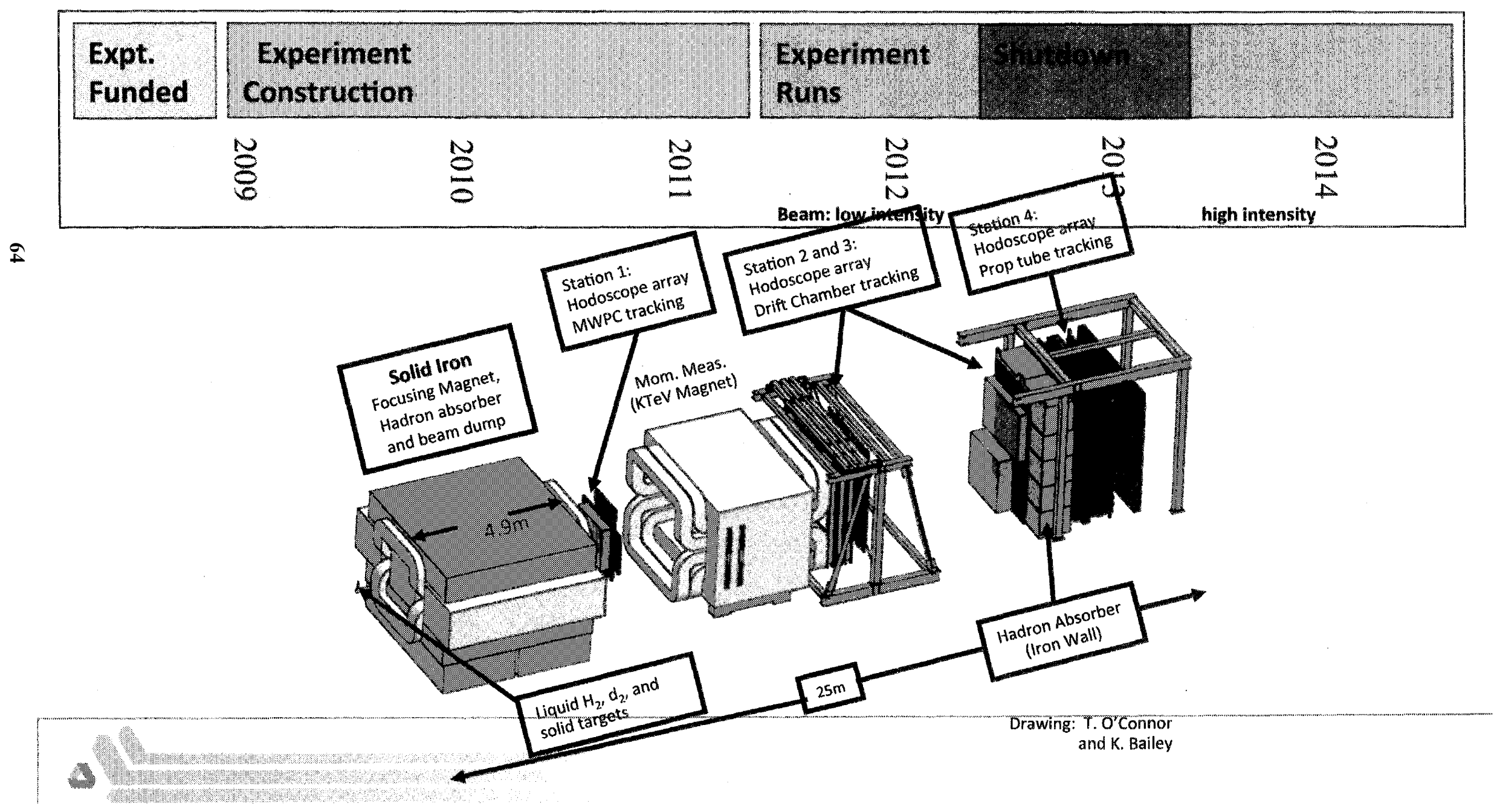




\section{At RHIC?}

- Internal Cluster-jet or pellet target $10^{15}$ atoms $/ \mathrm{cm}^{2}$...10\% beam.used for - 50 times thinner than RHIC CNI carbon target

- Operational modes

- Parasitic

- End-of-fill (HERMES)

- Dedicated (in-and-out strike)

- Other questions/obstacles

- Competition for interaction region (AnDY,

collider \& internal collider \& internal-

arget parasitic ru

$\sim 6$ hours

- Beam compensation for double dipole spectrometer

- Beam pipe through spectrometer?

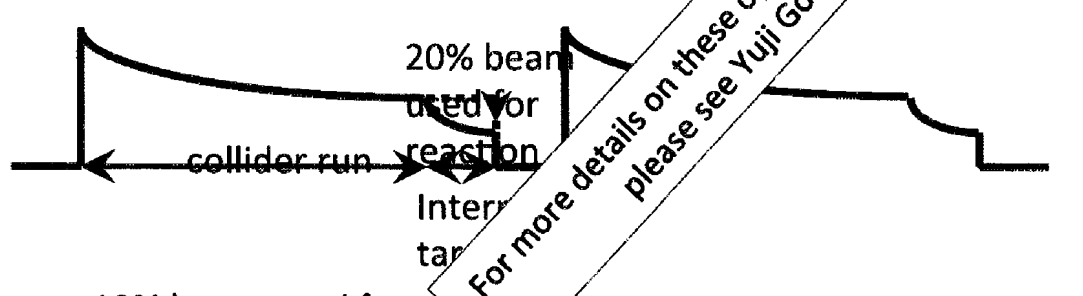

Measure not only the
sign of the Sivers but
also the shape of the
function
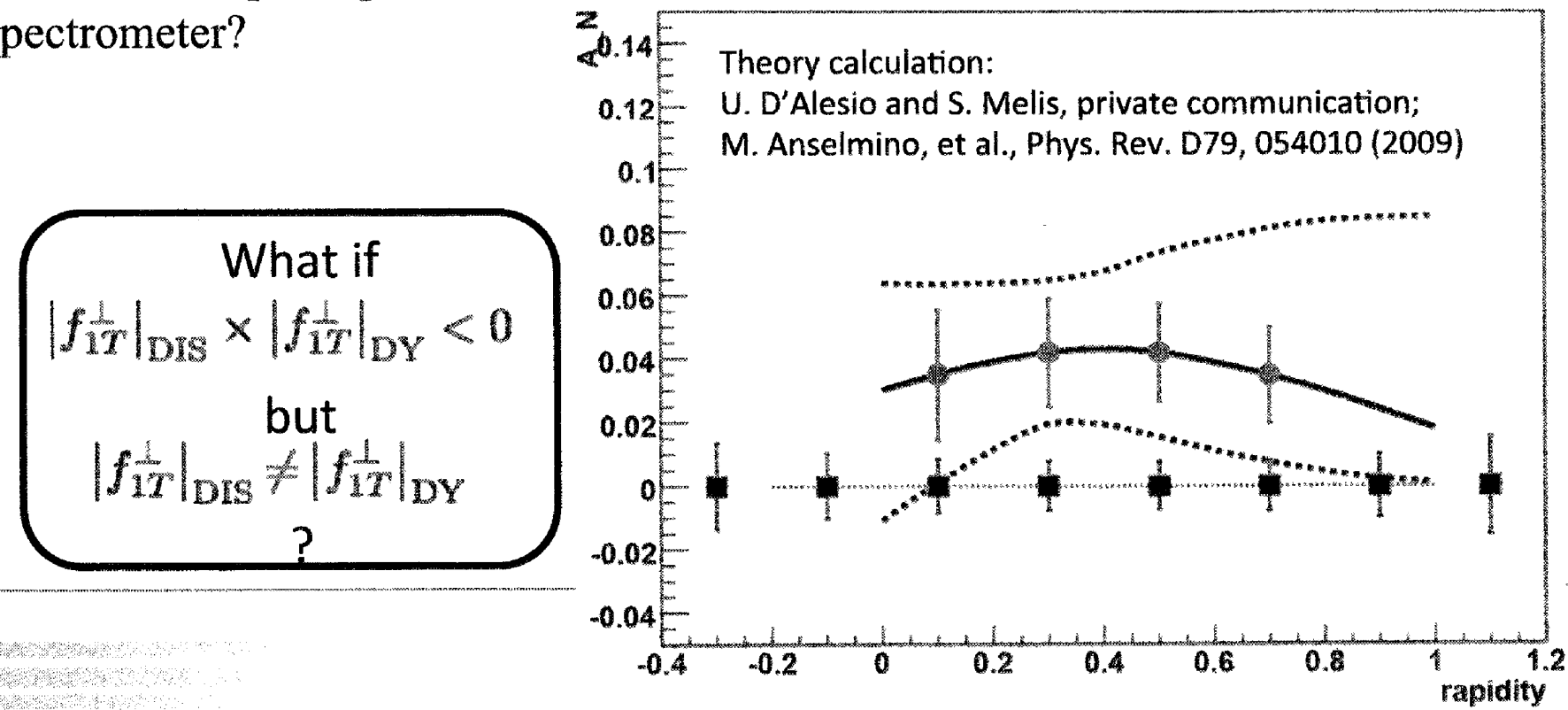


\section{Polarized beam at Fermilab Main Injector}

- $1 \mathrm{~mA}$ at polarized source delivers $8.1 \times 10^{11} \mathrm{p} / \mathrm{s}(=130 \mathrm{nA})$

- A. Krisch: Spin@Fermi study in 1995

- Fermilab Main Injector can be polarized (not Tevatron)

- Revisit study to re-evaluate cost (done in early fall 2011)

- Feasibility depends on cost (both in $\$ \$$ and down time of MI)

- Scenarios:

- SeaQuest liquid $\mathrm{H}_{2}$ target can take $\sim 5 \times 10^{11} \mathrm{p} / \mathrm{s}(=80 \mathrm{nA})$

$-\mathcal{L}=1 \times 10^{36} / \mathrm{cm}^{2} / \mathrm{s} \quad(60 \%$ of beam delivered to experiment $)$

\& $-\mathcal{L}=2 \times 10^{35} / \mathrm{cm}^{2} / \mathrm{s} \quad(10 \%$ of beam delivered to experiment $)$

- $x$-range:

$x_{1} 0.3-0.9$ (valence quarks)

$x_{2} 0.1-0.5$ (sea quarks)

- Unpolarized SeaQuest

- luminosity: $\mathcal{L}=3.4 \times 10^{35} / \mathrm{cm}^{2} / \mathrm{s}$

$-\mathrm{I}_{\mathrm{av}}=1.6 \times 10^{11} \mathrm{p} / \mathrm{s}(=26 \mathrm{nA})$

$-\mathrm{N}_{\mathrm{p}}=2.1 \times 10^{24} / \mathrm{cm}^{2}$

$-2-3$ years of running: $3.4 \times 10^{18}$ pot
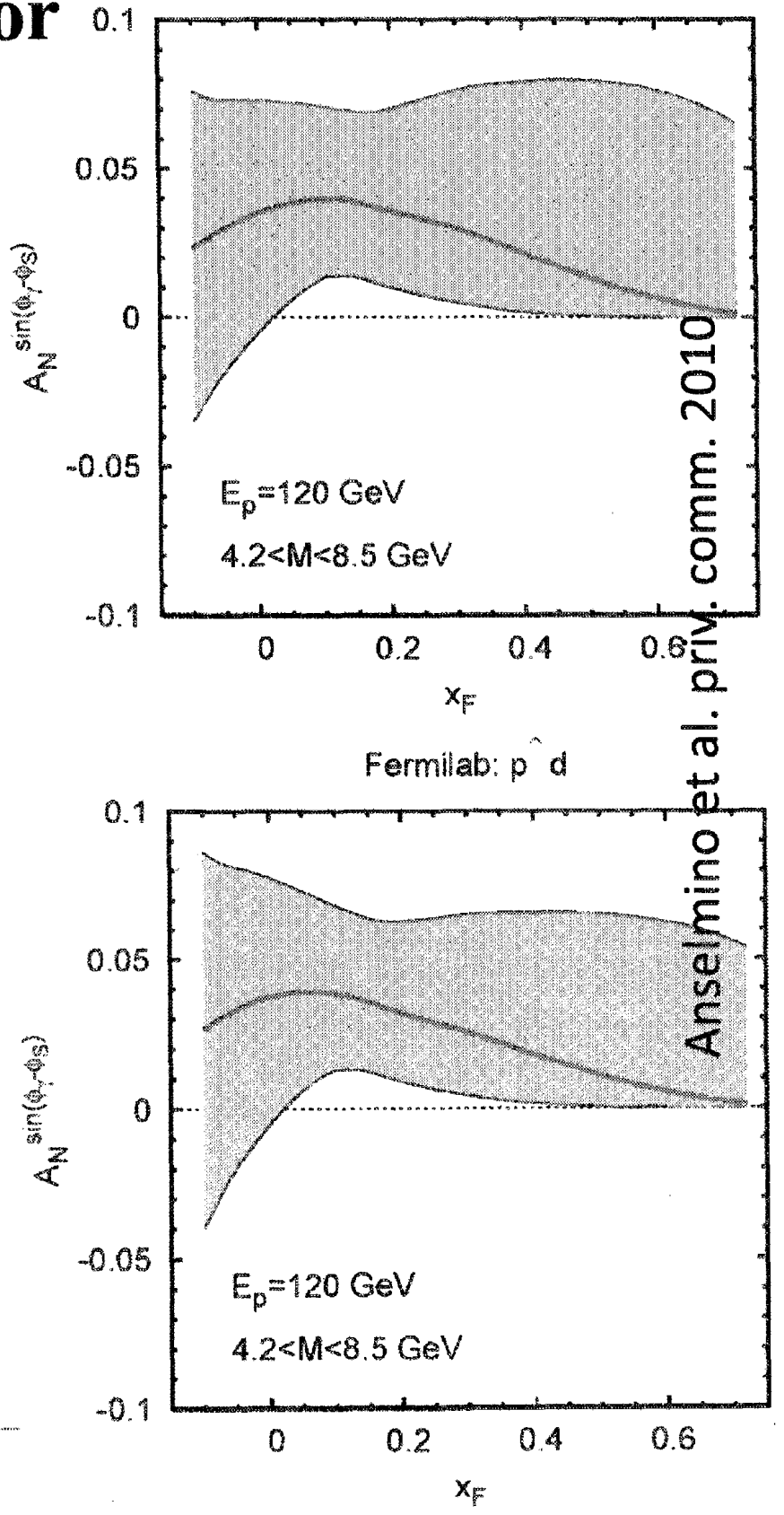


\begin{tabular}{|c|c|c|c|c|c|}
\hline 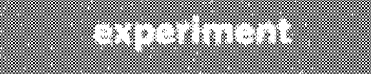 & Fortaros: & $00044^{2}$ & 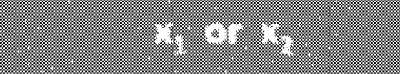 & wuthesh & rusches: \\
\hline $\begin{array}{l}\text { compass } \\
\text { (cERN) }\end{array}$ & $\pi^{2}+p$ & $\begin{array}{l}160 \mathrm{GeV} \\
\sqrt{5}=17.4 \mathrm{GeV}\end{array}$ & $\begin{array}{l}x_{2}=0.2-0.3 \\
x_{2}=0.05 \text { (low mass) }\end{array}$ & $2 \times 10^{-3} \mathrm{~cm}^{2} \mathrm{~s}^{4}$ & 32012 \\
\hline $\begin{array}{l}\text { PAX } \\
(G 5)\end{array}$ & $p^{*}+p_{p s t}$ & $\begin{array}{l}\text { collider } \\
\sqrt{s}=14 \mathrm{GeV}\end{array}$ & $x_{1}=0.1-0.9$ & $2 \times 10^{30} \mathrm{~cm}^{2} \mathrm{~s}^{-1}$ & $>2017$ \\
\hline $\begin{array}{l}\text { PANDA } \\
\text { (GSO) }\end{array}$ & $p_{j}+p^{2}$ & $\begin{array}{l}15 \mathrm{GeV} \\
\sqrt{\mathrm{s}}=5.5 \mathrm{GeV}\end{array}$ & $x_{2}=0.2-0.4$ & $2 \times 10^{-2} \mathrm{~cm}^{2} \mathrm{~s}^{-1}$ & 2016 \\
\hline JPARC & $p+p$ & $\begin{array}{l}50 \mathrm{GeV} \\
\sqrt{s}=10 \mathrm{GeV}\end{array}$ & $x_{1}=0.5-0.9$ & $1 \times 10^{35} \mathrm{~cm}^{22} \mathrm{~s}^{-3}$ & $>2015 ? ?$ \\
\hline $\begin{array}{l}\text { NICA } \\
\text { (MNR) }\end{array}$ & $p^{t}+p$ & $\begin{array}{l}\text { colluer } \\
v_{s}=20 \mathrm{GeV}\end{array}$ & $x_{1}=0.2-0.8$ & $1 \times 10^{6} \mathrm{~cm}^{2} \mathrm{~s}^{3}$ & $\geq 2014$ \\
\hline $\begin{array}{l}\text { PHENXX } \\
\text { (RHIC) }\end{array}$ & $p^{t}+p$ & $\begin{array}{l}\text { collicer } \\
\sqrt{5}=500 \mathrm{GeV}\end{array}$ & $x_{1}=0.05-0.1$ & $2 \times 10^{32} \mathrm{~cm}^{-2} \mathrm{~s}^{-2}$ & $>2018$ \\
\hline $\begin{array}{l}\text { RHic mtemal } \\
\text { target phase-1. }\end{array}$ & $p^{t}+\rho$ & $\begin{array}{l}250 \mathrm{GeV} \\
\mathrm{s}=22 \mathrm{GeV}\end{array}$ & $x_{1}=0.25-0.4$ & $2 \times 10^{-1} \mathrm{~cm}^{2} \mathrm{~s}^{2}$ & 32018 \\
\hline $\begin{array}{l}\text { RHIC internal } \\
\text { target phase-1 }\end{array}$ & $p^{t}+p$ & $\begin{array}{l}250 \mathrm{GeV} \\
\sqrt{s}=22 \mathrm{GeV}\end{array}$ & $x_{1}=0.25-0.4$ & $6 \times 10^{34} \mathrm{~cm}^{-2} \mathrm{~s}^{2}$ & $>2018$ \\
\hline $\begin{array}{l}\text { A. } \mathrm{UY} \\
\text { RHIC }\left(\mathrm{P}^{2}-2\right)\end{array}$ & $p^{3}+\frac{1}{2}$ & $\begin{array}{l}500 \mathrm{GeV} \\
y_{5}=32 \mathrm{GeV}\end{array}$ & $x_{1}=?$ & $? ? \mathrm{~cm}^{2} \mathrm{~s}^{3}$ & $>2015$ \\
\hline $\begin{array}{l}\text { SeaQuest (unpol.) } \\
\text { (FNAL) }\end{array}$ & $p+p$ & $\begin{array}{l}120 \mathrm{GeV} \\
\sqrt{5}=15 \mathrm{GeV}\end{array}$ & $x_{1}=0.3-0.9$ & $3.4 \times 10^{35} \mathrm{~cm}^{2} \mathrm{~s}^{2}$ & $>2010$ \\
\hline $\begin{array}{l}\text { pol. SeaQuest } \\
\text { (FNAL) }\end{array}$ & $p^{2}+2$ & $\begin{array}{l}120 \mathrm{GeV} \\
\mathrm{is}=15 \mathrm{GeV}\end{array}$ & $x=0.3$ & $1 \times 10^{4} \mathrm{~cm}^{2} \mathrm{~s}$ & $3 ?$ \\
\hline
\end{tabular}




\section{Drell-Yan at Fermilab}

What is the structure of the nucleon?

- What is d-bar/u-bar?

- What are the origins of the sea quarks?

- What is the high-x structure of the proton?
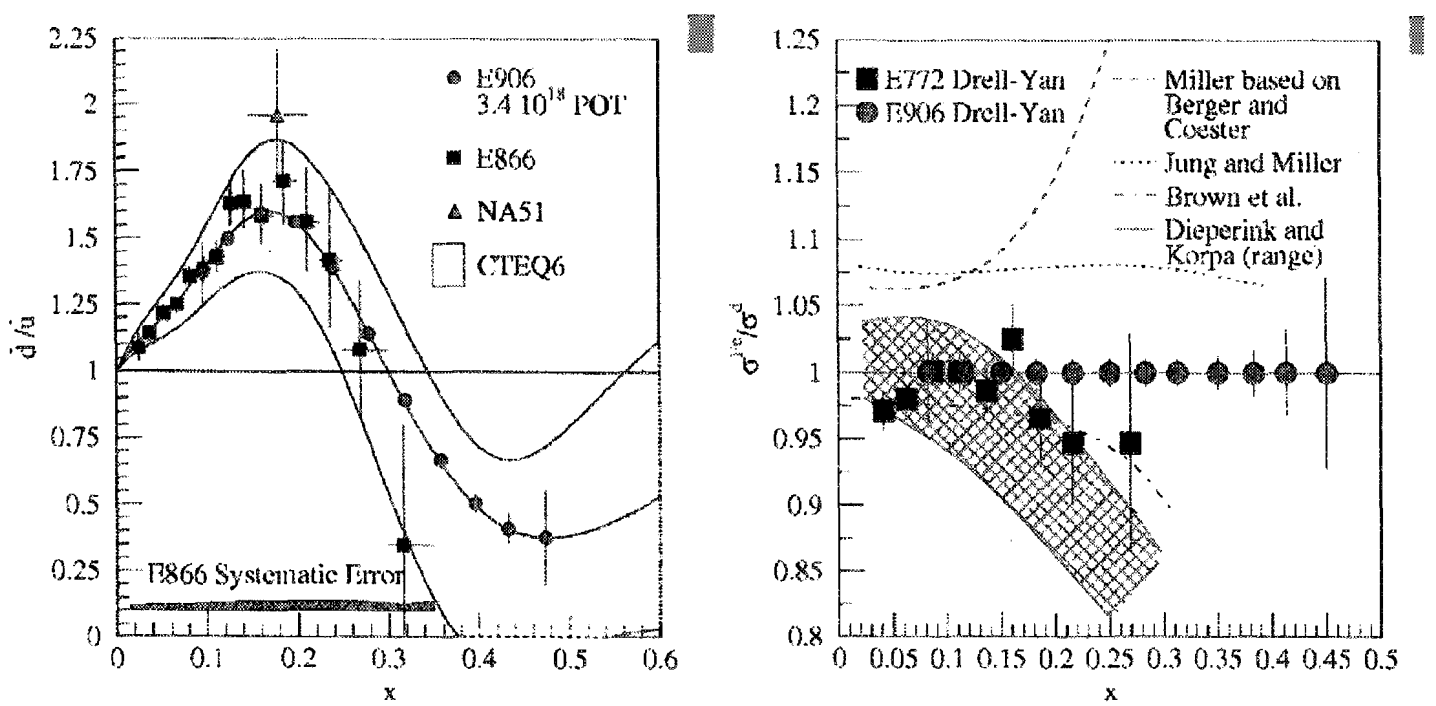

What is the structure of nucleonic matter?

- Where are the nuclear pions?

क - Is anti-shadowing a valence effect?

What is the transverse Structure of the proton?

- Polarized beam at Fermilab Main Injector

- Move apparatus to RHIC or J_PARC

This work is supported in part by the U.S. Department of Energy, Office of Nuclear Physics, under Contract No. DE-AC02-06CH11357.
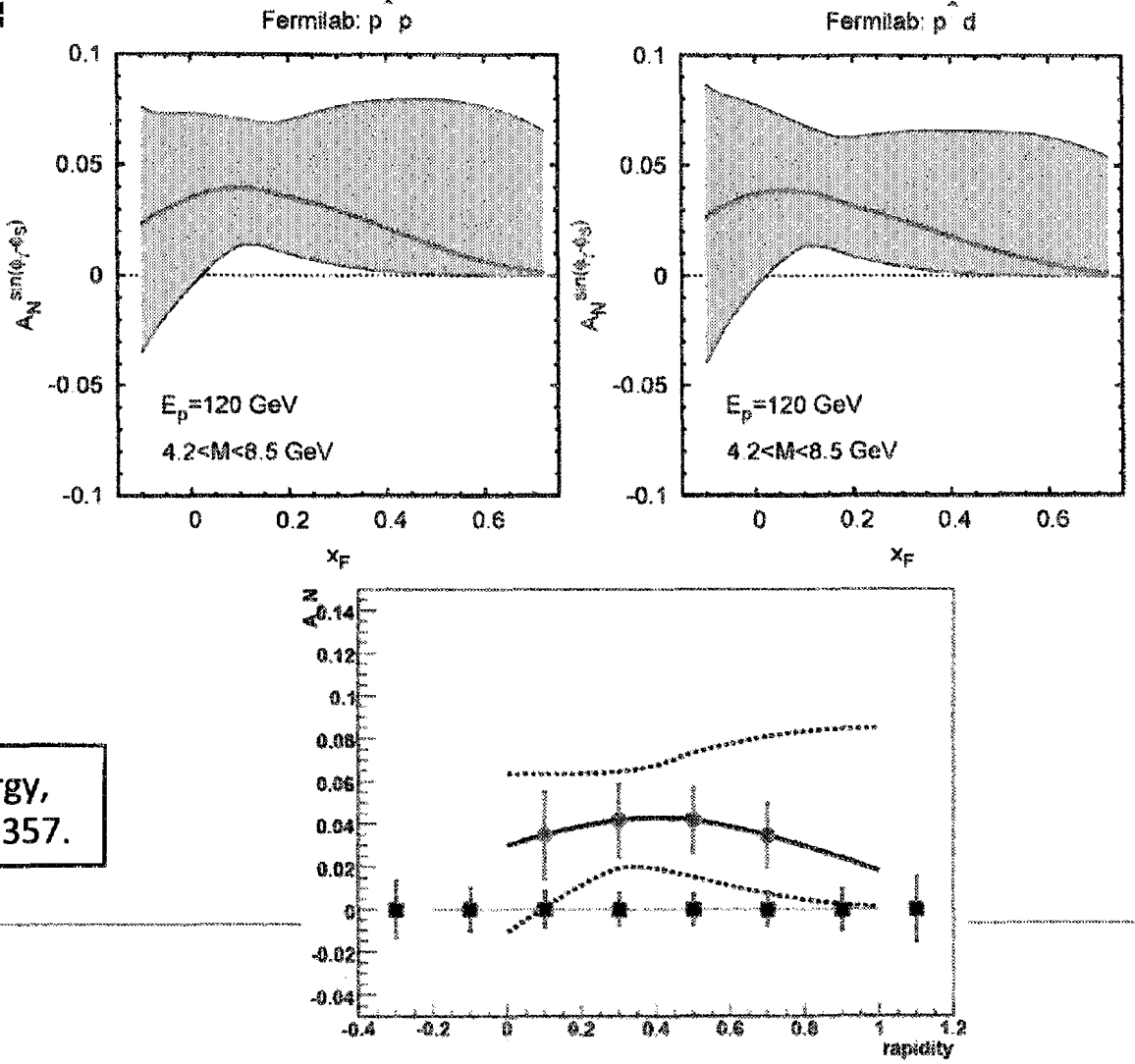


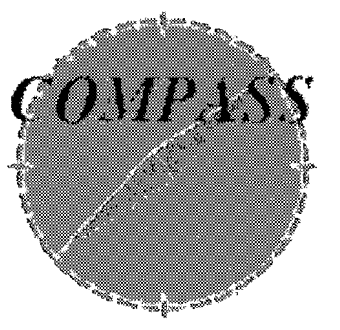

Dr. Oleg Denisov, INFN section of Turin, Italy

\section{Forthcoming Drell-Yan experiment at COMPASS}

The COMPASS experiment at CERN is a universal facility which can operate with both muon and hadron beams as well as with the longitudinally/transversely polarized solid target. The main goal of the experiment is to study the spin structure of the nucleon. The availability of hadron(pion) beam provides an access to the Drell-Yan physics, i.e. to the process where quark(target)antiquark(beam) pair annihilates electromagnetically with a production of dilepton pair. Study of angular dependencies of the DrellYan process cross-section allows us to access parton distribution functions (PDFs) or, more precisely, a convolutions of various PDFs. The possibility to use in a future COMPASS Drell-Yan experiment a transversely polarized target together with negative pion beam will provides us with unique data on transverse momentum dependent (TMD) PDFs.

The COMPASS-II proposal [1], which includes the single-polarized Drell-Yan measurements, was submitted to the CERN SPS committee in May 2010, was recommended by SPSC for approval in September 2010 and approved by CERN research board in December 2010. In this presentation the most important features of the Drell-Yan experiment at COMPASS will be discussed. The experimental set-up, its performance including apparatus acceptance and kinematic range coverage as well as projections for the achievable statistical errors on various single-spin-asymmetries will also be reported.

[1] COMPASS Coll., COMPASS-II proposal, CERN-SPSC-2010-014, SPSC-P-340, May 172010 


\section{COMPASS facility at CERN}

WFN

COMPASS

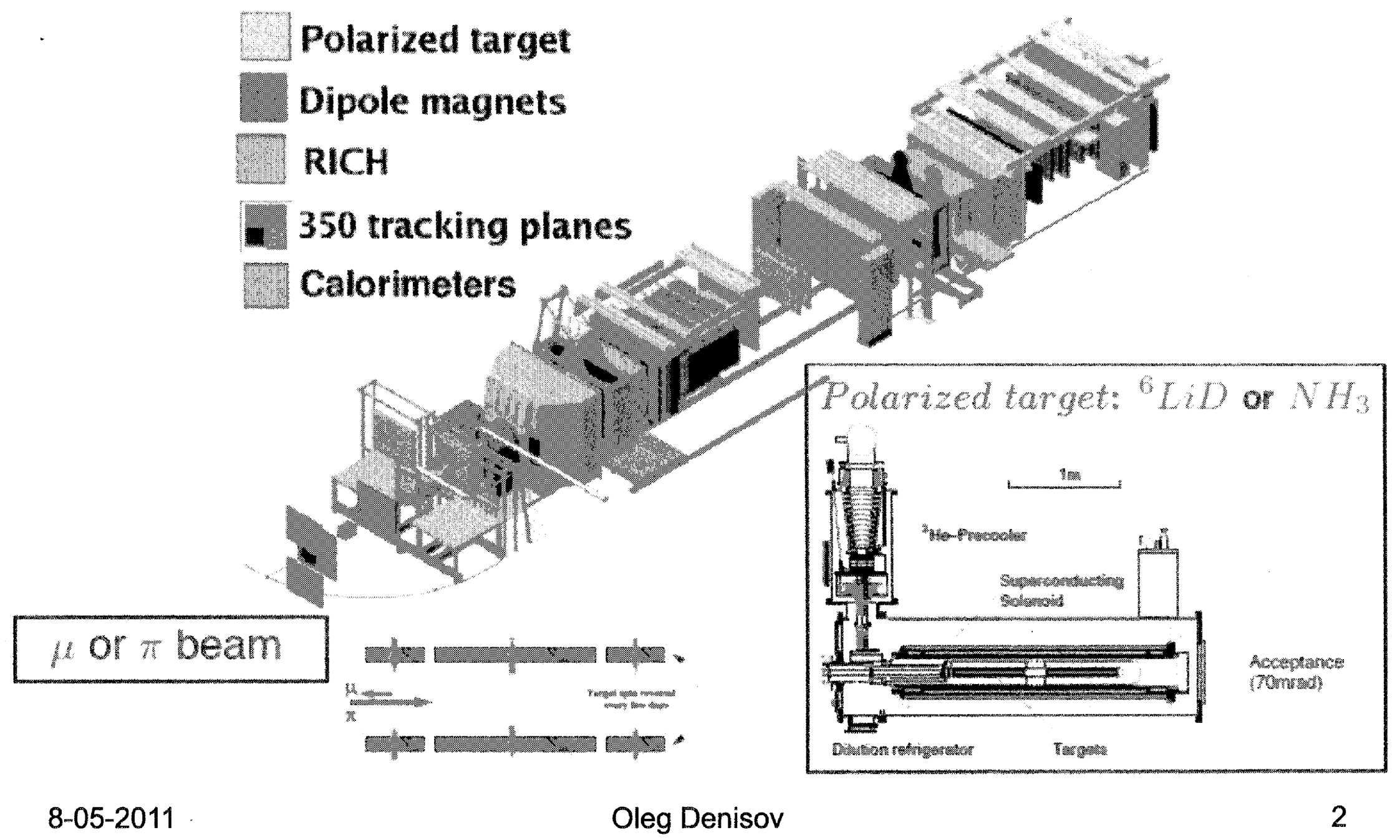




\section{COMPASS-II (New Physics) a piece of history}

- COMPASS is very sophisticated, universal and flexible facility $\rightarrow$

Physics beyong SIDIS and hadron spectroscopy is possible:

- Unique COMPASS Polarised Target

- Both hadron and lepton beams

- Easy-accessable spectrometer components

- All that all together has generated new physics proposals with COMPASS - DVCS(GPDs) and polarised DY:

- For the first time these ideas (GPD and DY) were reported at the Villars SPSC meeting in September 2004

- Since then (DY part) 3 International Workshops (Torino, Dubna, CERN), $>40$ COMPASS DY subgroup meetings, 3 Beam Tests, $>20$ presentations at the international Conferences....

- The COMPASS $I I$ proposal is submitted to the SPSC on May $17^{\text {th }}$ 2010

- Approved by the CERN research board on December 1 st 2010

- April $7^{\text {th }}$ - the Collaboration took a decision to run first the DY program and then DVCS (GPDs) program - we will start in 2013 (beam test) and in 2014 we will have a full year of DY data taking. 


\section{COMPASS-II: a Facility to study QCD}

COMMON

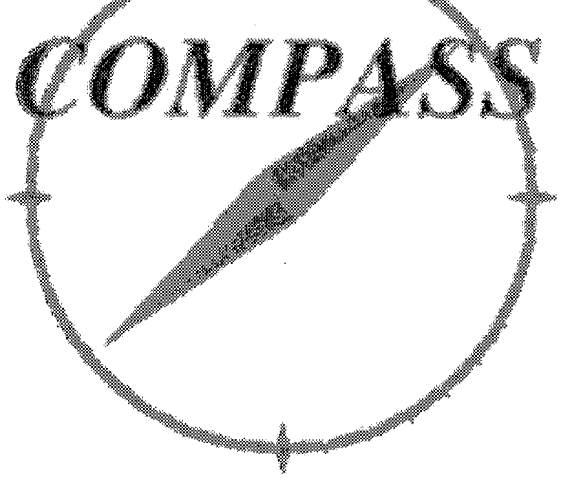

MUON and

PROTON

APPARATUS for

STRUCTURE and SPECTROSCOPY

$\therefore$ Long Term Plans for at least 5 years (starting in 2012)

$\checkmark$ Primakoff with $\pi, K$ beam $\rightarrow$ Test of Chiral Perturb. theory

$\checkmark$ DVCS \& DVMP with $\mu$ beams $\rightarrow$ Transv. Spatial Distrib. with GPDs

$\checkmark$ SIDIS (with GPD prog.) $\rightarrow$ Strange PDF and Transv. Mom. dep. PDFs

Drell-Yan with $\pi$ beons $\rightarrow$ Transverse Momentum dependent PDFs

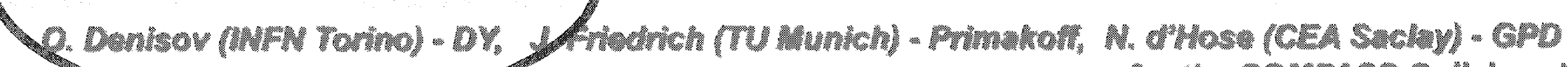

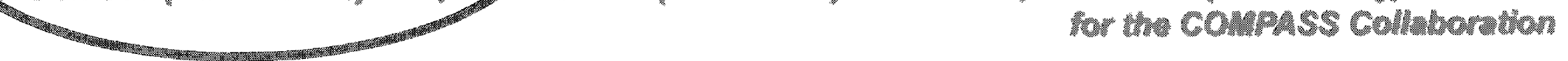




\section{$\pi p \rightarrow$}

- In our case $\left(\pi^{-} p \rightarrow \mu^{-} \mu X\right)$ contribution from valence quarks is dominant

- In COMPASS kinematics uubar dominance

- $\quad\left\langle\mathrm{P}_{\mathrm{T}}>\sim 1 \mathrm{GeV}\right.$ - TMDs induced effects expected to be dominant with respect to the higher QCD corrections
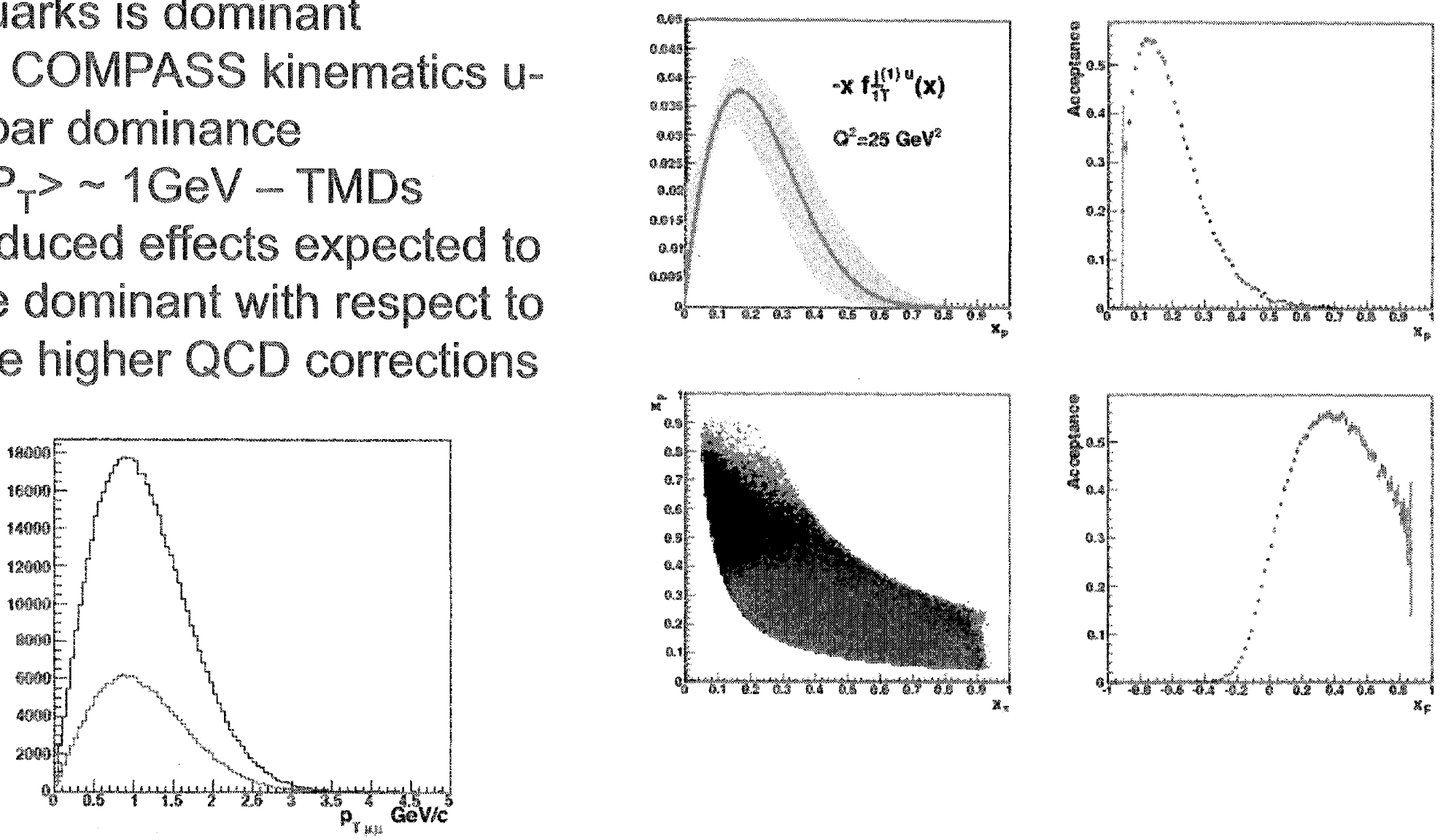


$$
(\mathrm{HMR}): 4 . \leq M_{\mu \mu} \leq 9 . \mathrm{GeV} / \mathrm{c}^{2}
$$
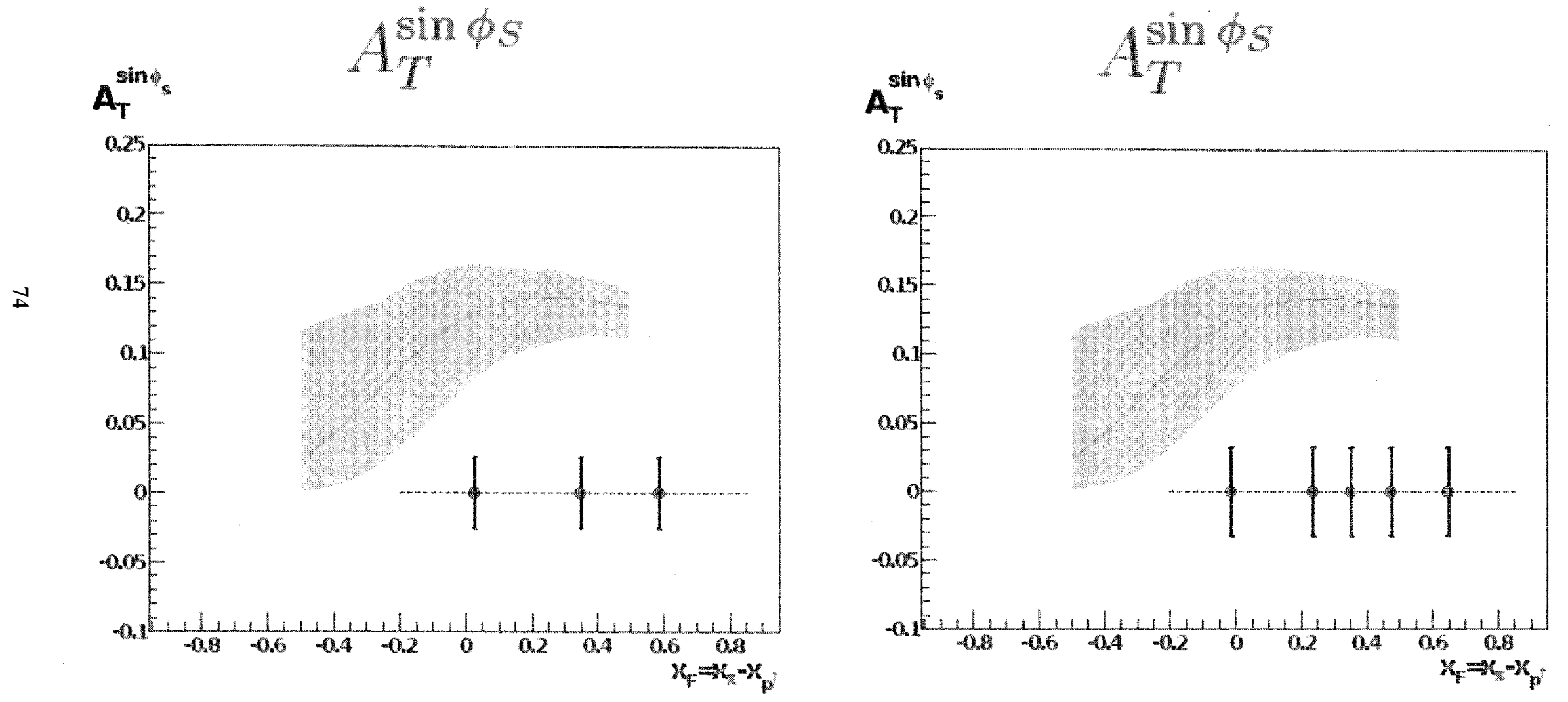


\title{
Other Physics Opportunities in Future Drell-Yan Experiments
}

\author{
Jen-Chieh Peng \\ University of Illinois at Urbana-Champaign
}

Workshop on "Opportunities for Drell-Yan Physics at RHIC" BNL, May 11-13, 2011

\section{Outline}

- "Intrinsic sea-quarks" of the nucleons.

- Flavor dependence of the EMC effect.

- Equalities and inequalities in Drell-Yan azimuthal angular distributions.

- Flavor and $x$-dependence of quark intrinsic transverse momentum distributions.

- Drell-Yan and quarkonium duality. 

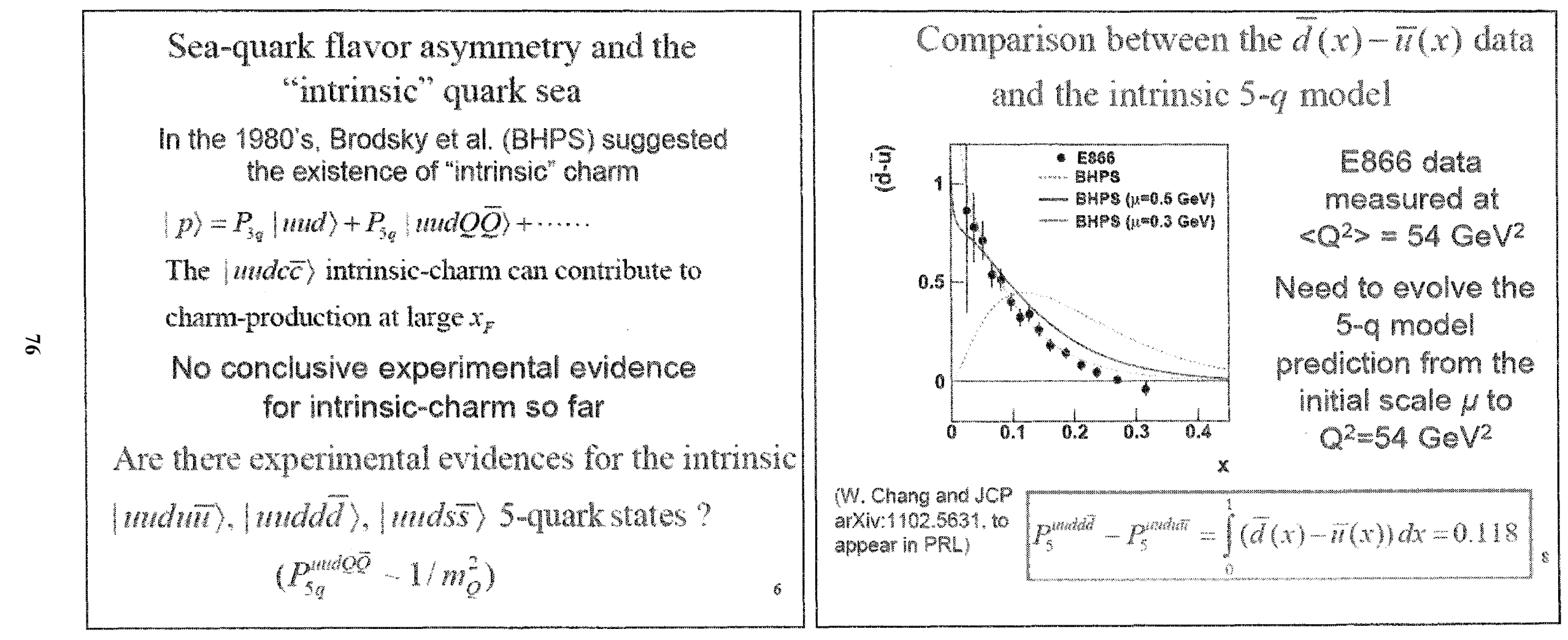


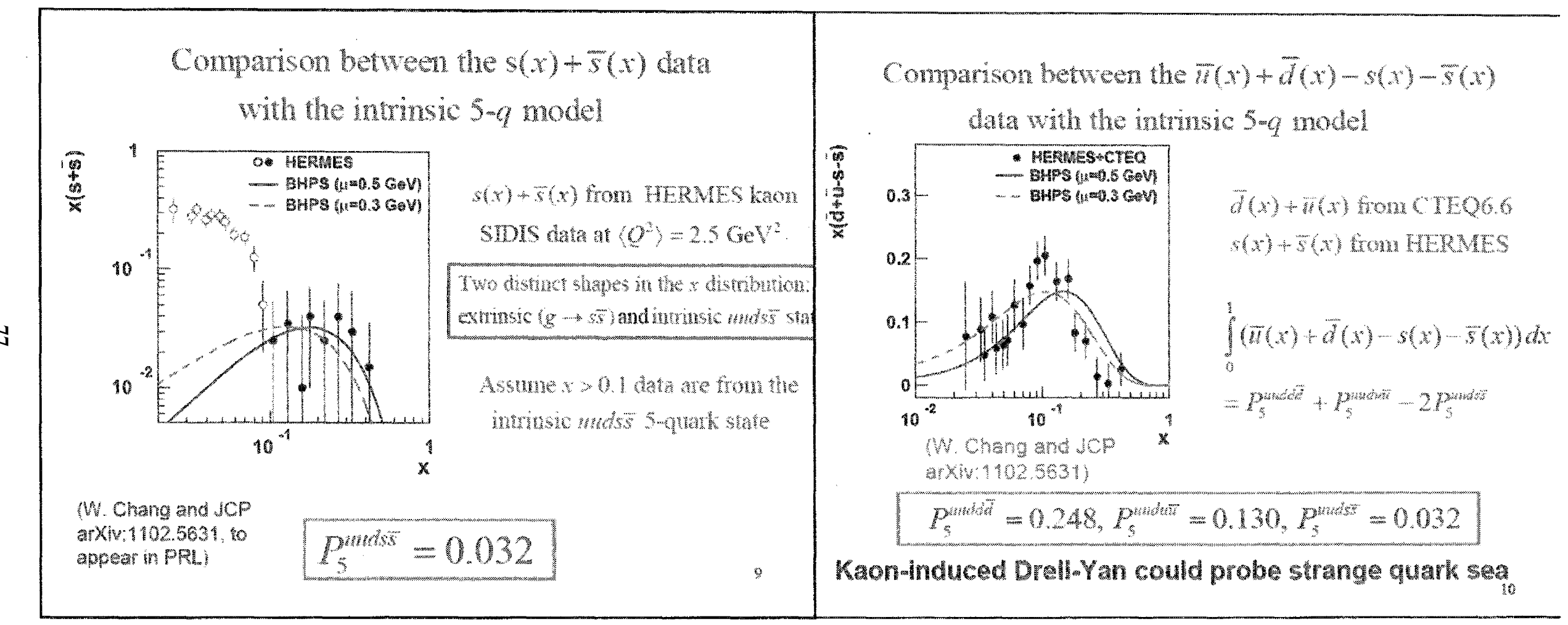



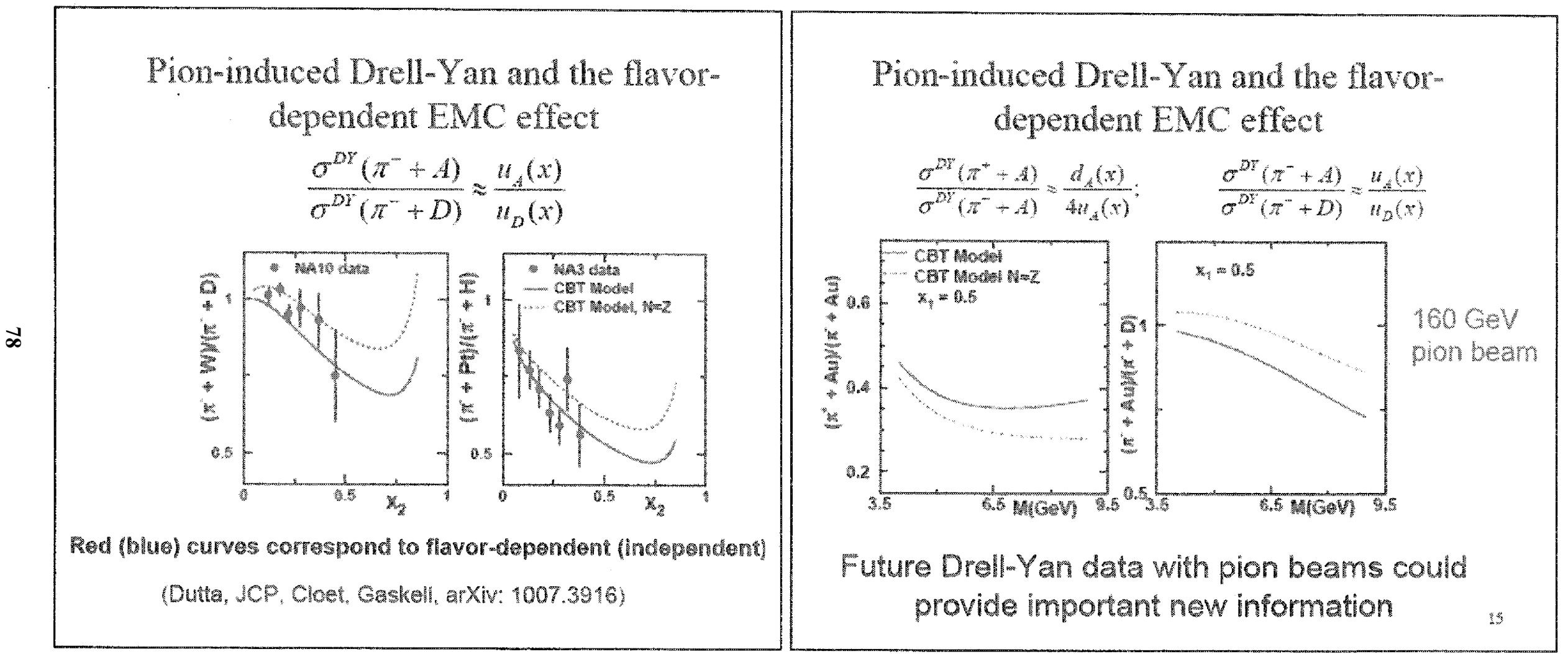
Results on $\cos 2 \phi$ Distribution in $p+p$ Drell-Yan

L. Zhu, J.C. Peng, et al., PRL 102 (2009) 182001

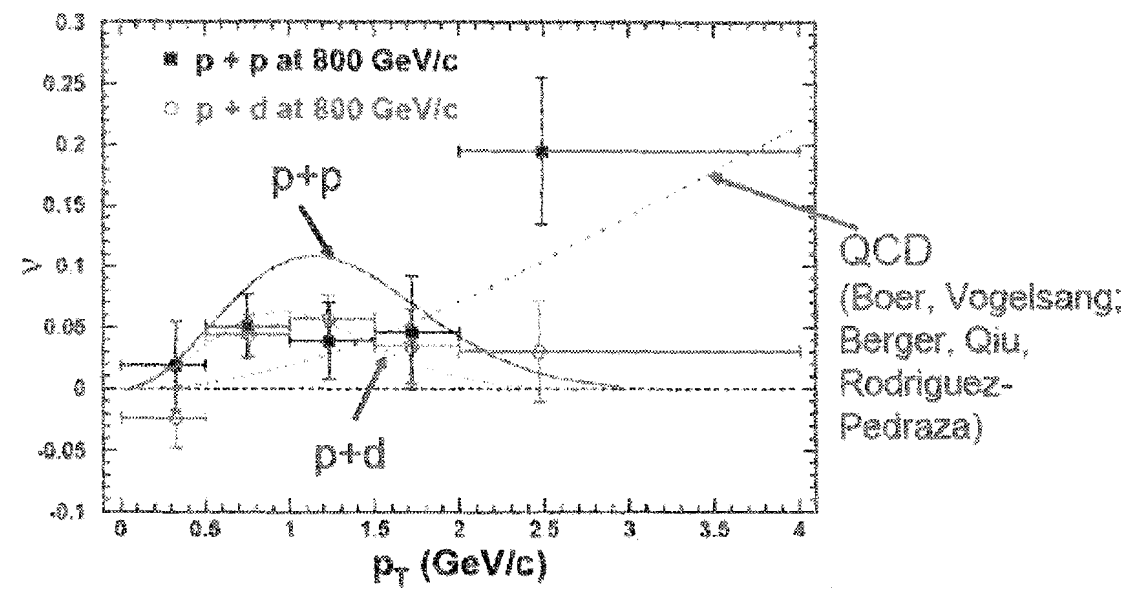

Combined analysis of S101S and D-Y by Melis et al.

More data are anticipated from future DY exps.
Is the $\mu^{2} \leq(1-\lambda)(1+\lambda-v) / 4$ inequality valid?

$$
(1-\lambda)(1+\lambda-V) / 4-\mu^{2} \geq 0 ?
$$

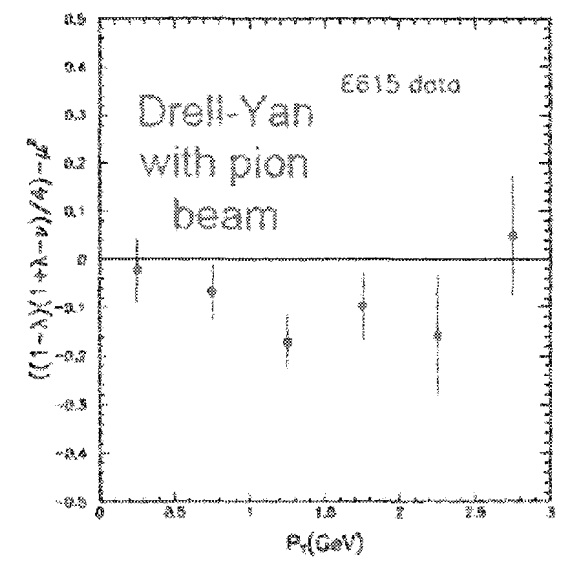

The inequality appears to be viclated

Teryaev and $J \mathrm{cP}$ )

Our knowledge of D-Y azimuthal angular dependence is still incomplete (New Drell-Yan data are essential) 
What do we know about the quark and gluon transverse momentum distributions?

- Does the quark $\mathrm{k}_{\mathrm{T}}$ distribution depend on $x$ ?

- Do valence quarks and sea quarks have different $k_{T}$ distributions?

- Do $u$ and $d$ quarks have the same $k_{T}$ distribution?

- Do nucleons and mesons have different quark $k_{T}$ distribution?

- Do gluons have $k_{T}$ distribution different from quarks?

- Important for extracting the TMD parton distributior - Interesting physics in its own right

\section{Possible $x$-dependent $k_{T}$ distributions} $E 866 \mathrm{p}+\mathrm{d}$ D.Y data ( $800 \mathrm{GeV}$ beam)
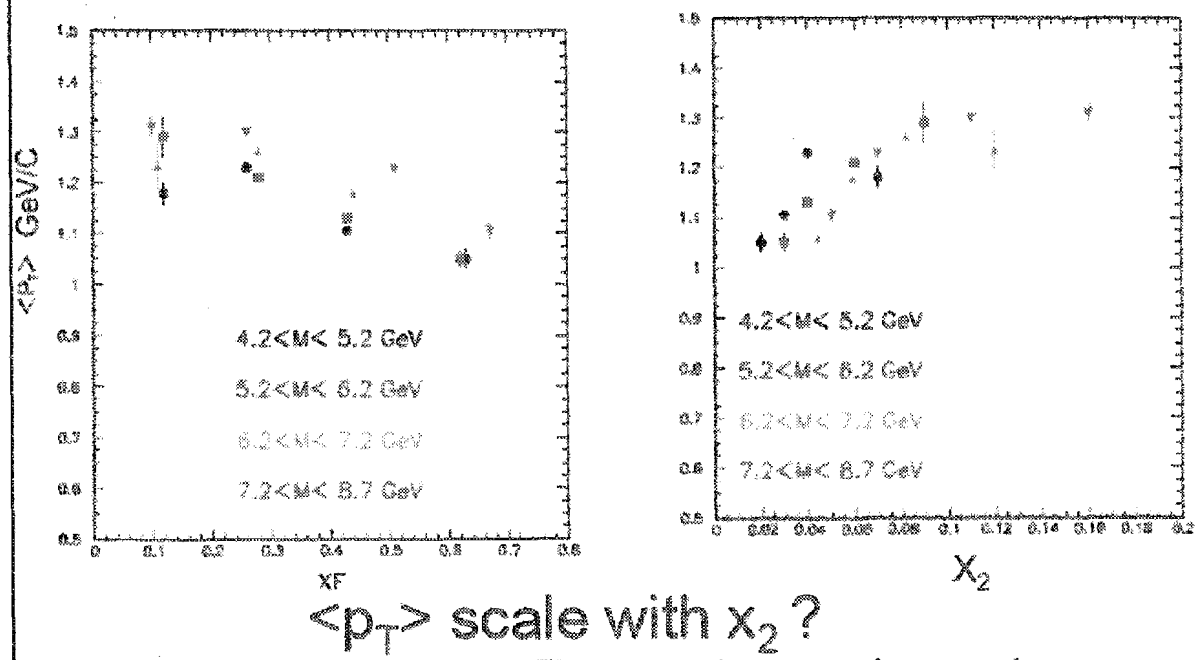

Analysis is ongoing. Future data at lower beam energies are essential 


\title{
Drell-Yan Production at STAR Status and Plans
}

\author{
Ernst Sichtermann (LBNL) \\ for the STAR Collaboration
}

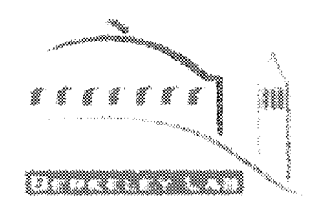

Opportunities for Drell-Yan Physics at RHIC RIKEN BNL Research Center Workshop May 11-13, 2011 at Brookhaven National Laboratory

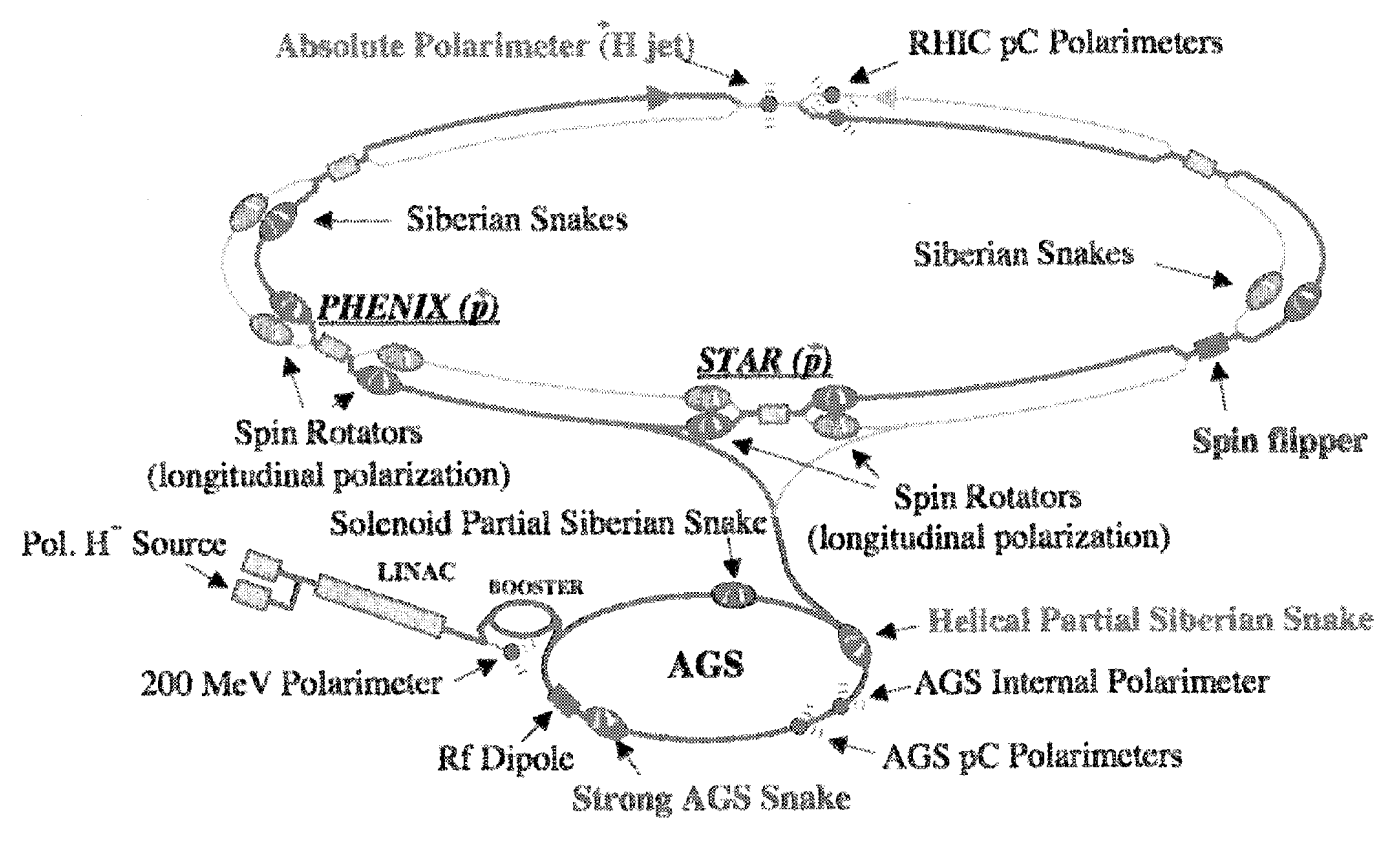




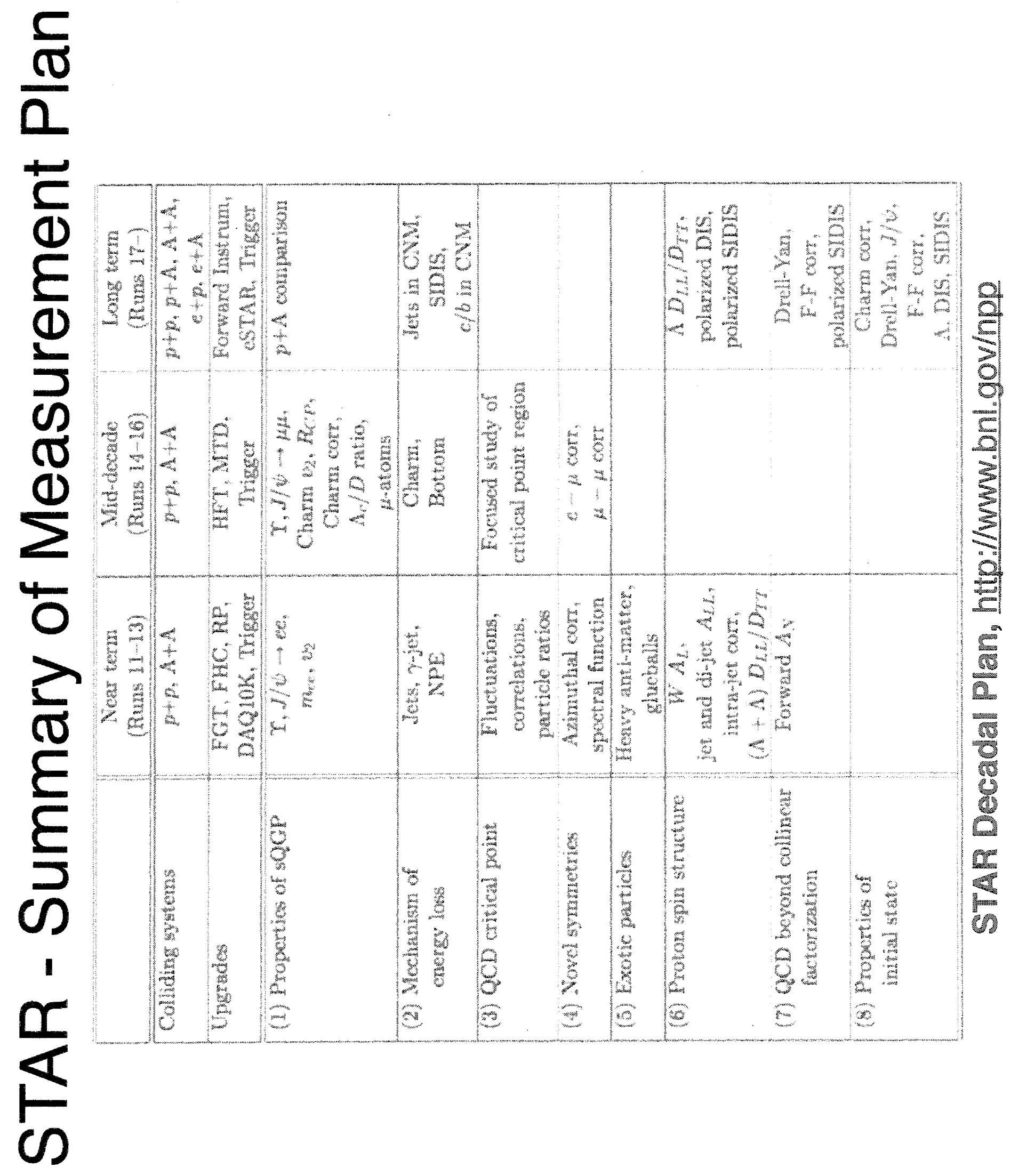




\section{STAR Experiment - Forward Calorimeters}

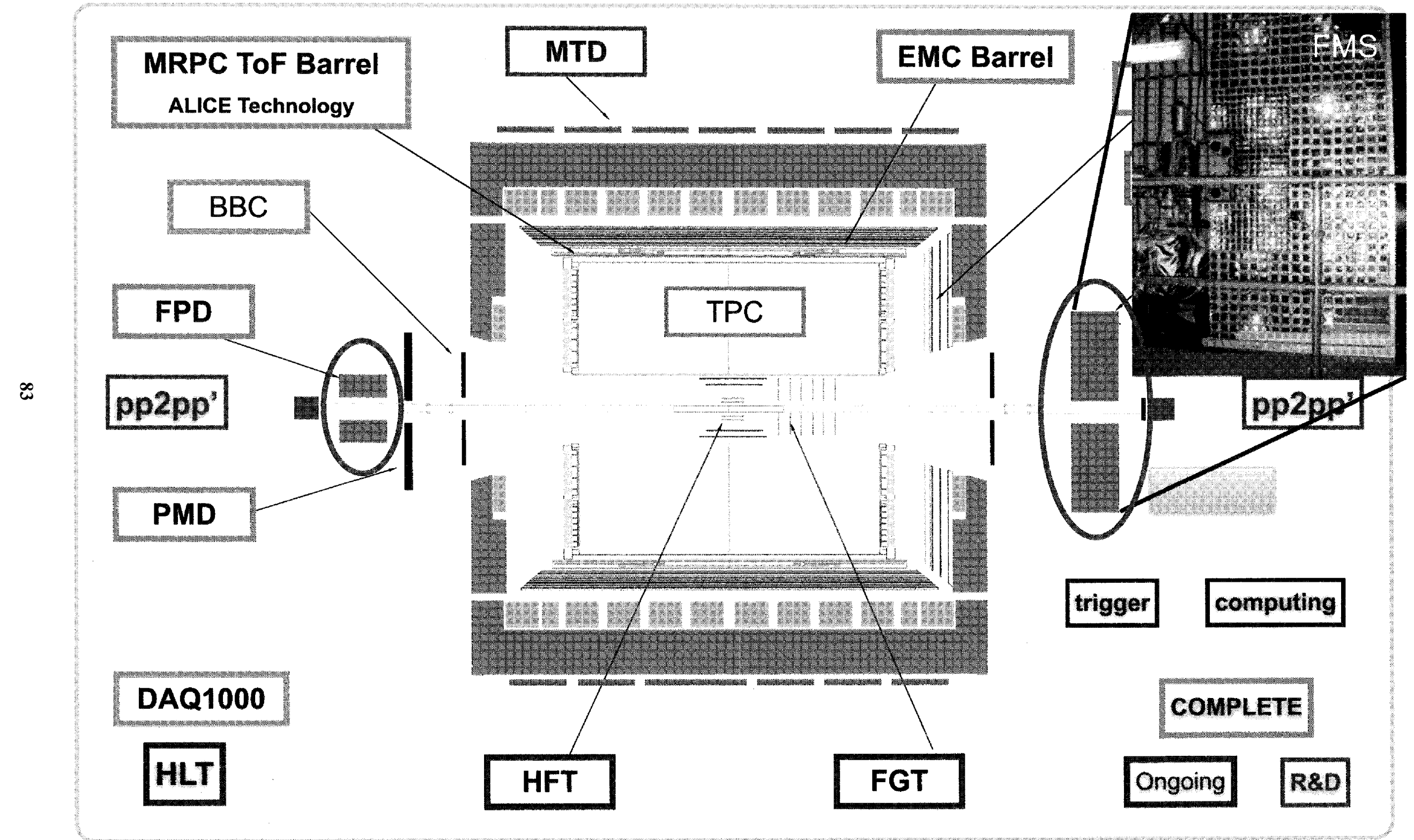




\section{A Possible Future Upgrade at STAR}

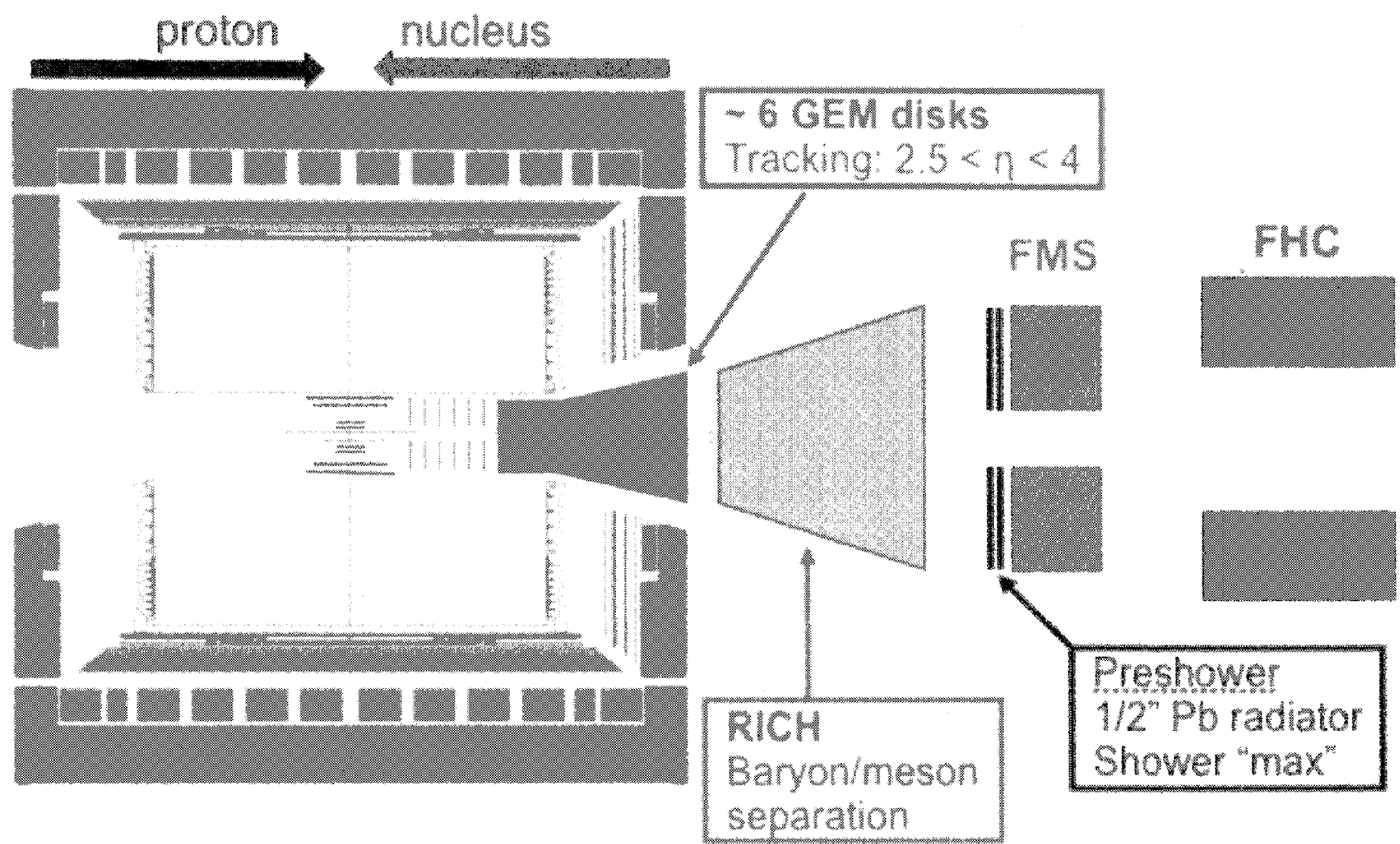

Forward upgrade driven by proton-nucleus and transverse spin physics considerations,

- charged particle tracking,

- electron-hadron and photon-neutral pion separation,

- Baryon meson separation.

Optimizations and full simulations to demonstrate capability are starting. 


\section{Drell-Yan, eRHIC, eSTAR}

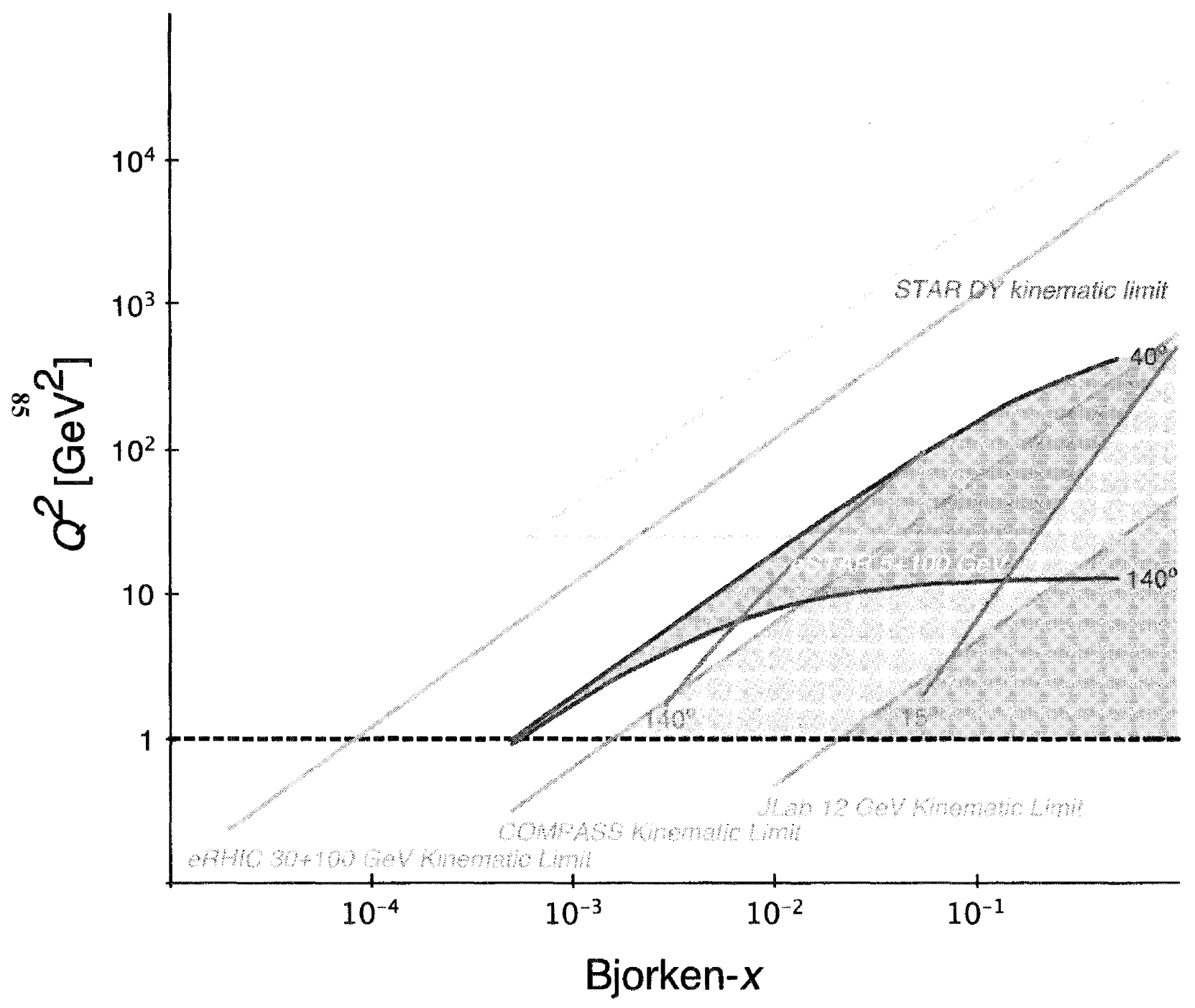

A talk by itself...

Note: this is an illustration, not a full simulation.

Here, $M>5 \mathrm{GeV}$ for DY, central-rapidity for eSTAR 


\section{Concluding Remarks}

STAR has prepared a new decadal plan for 2011-2020, http://www.bnl.gov/npp

Aims to address transverse physics and nuclear structure physics topics via DrellYan measurements in the second half of the decade, as part of a broader program that may culminate in an Electron-Ion-Collider,

The Forward Meson Spectrometer is a key part of this program, and has been very successfully commissioned and operated up to $\sqrt{s}=500 \mathrm{GeV}$,

Anticipate at the level of 150 Drell Yan pairs in the FMS acceptance at $\sqrt{ } \mathrm{s}=200$ $\mathrm{GeV}$, about equal for proton+nucleus and proton+proton collisions, based on RHIC projections,

$\sqrt{ } \mathrm{s}=500 \mathrm{GeV} p+p$ projected rates are considerably higher, however, detection at STAR will be considerably more challenging, $\mathrm{p}+\mathrm{A}$ collisions are not possible at this energy at $\mathrm{RHIC}$,

Lots of work ahead,

- a number of key aspects are well understood/benchmarked,

- the foreseen upgrade path is evolutionary,

- efforts towards full simulations of measurement capability are starting,

- continued R\&D, ...

Thank you! 


\title{
Drell-Yan Production at PHENIX Status and Plan
}

\author{
Ming Liu \\ Los Alamos National Lab \\ (PHENIX Collaboration)
}

Abstract:

We present the current status and plan for Drell-Yan measurements at PHENIX for next decade. Initial feasibility studies of Drell-Yan production have been carried out with the soon available central and forward vertex upgrade detectors in the dielectron and dimuon channels. Significant luminosity, order of $250 \mathrm{pb}^{-1}$, is required in order to test and confirm the sign change (or not) in transverse single spin asymmetry (TSSA) in Drell-Yan production in the forward muon arm coverage. In the next five years, PHENIX will carry out Drell-Yan measurements at least to benchmark the cross sections in the PHENIX central electron and forward muon arms covered rapidity ranges from the expected high luminosity longitudinally polarized 500 $\mathrm{GeV} p+p$ collisions as well as from transversely polarized $200 \mathrm{GeV} p+p$ runs.

Beyond the next 5 years, we have identified new areas of investigation related to the fundamental properties of the SQGP, and to transverse spin physics, that require major new detector capabilities. PHENIX has an ambitious upgrade plan to significantly improve physics capability in the very forward region. The proposed sPHENIX detectors will replace the current PHENIX central magnets with a compact solenoid; in the forward direction, one of the current muon arm (south arm) will be replaced with a new large-acceptance forward spectrometer $(\eta=2 \sim 4)$ with excellent PID for hadrons, electrons, and photons and full jet reconstruction capability. This will enable us for the future eRHIC physics also. Drell-Yan production and asymmetry will be studied in the di-electron channel in a very forward rapidity range that goes beyond the current PHENIX muon arm coverage where significant TSSA $A_{N}$ is expected. Detailed MC simulation work with sPHENIX is underway to study the experimental sensitivity to TSSA in Drell-Yan production. 

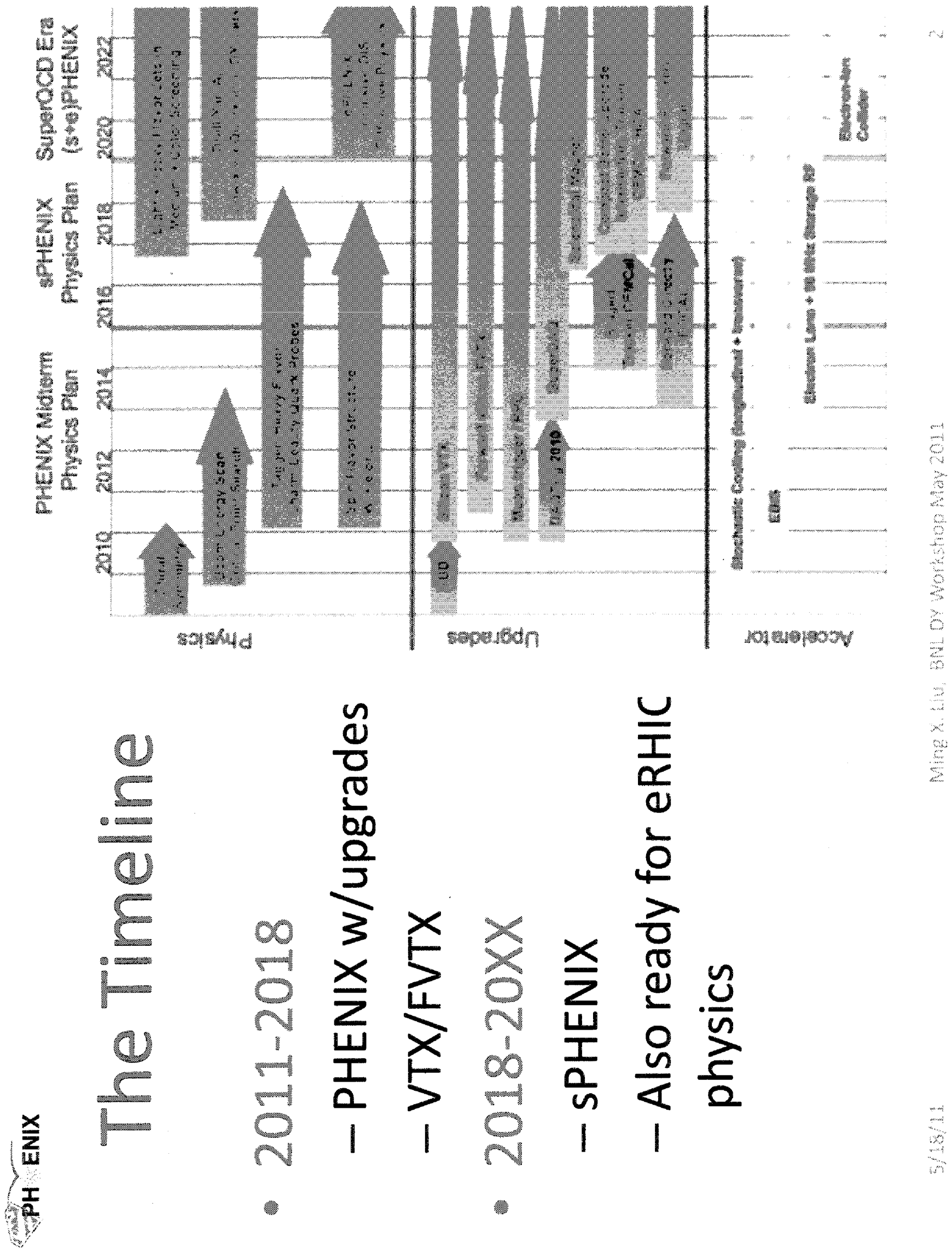


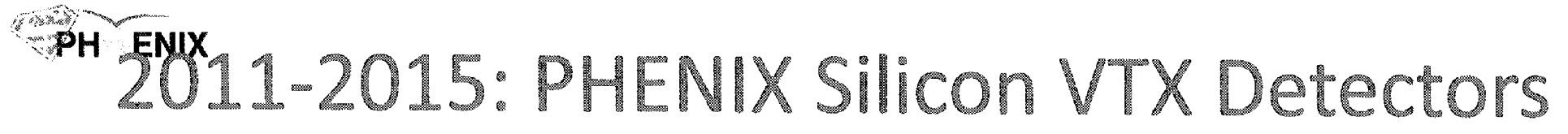

- upgrade on going, will be completed in 2011

- Precision Charm/Beauty Measurements

- $\mathrm{B} \rightarrow \mathrm{J} / \psi$, Drell-Yan, $\psi^{\prime}$
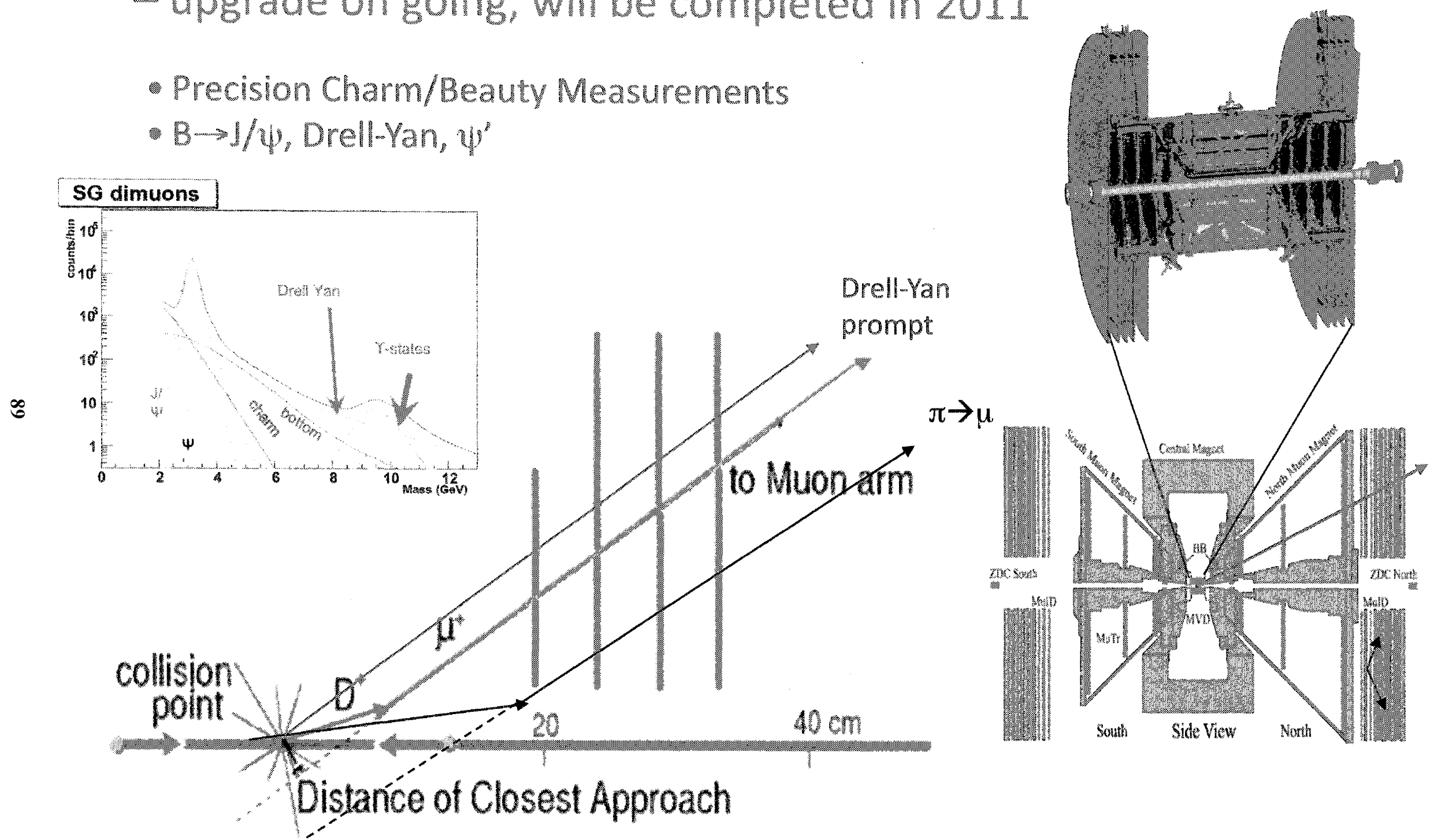


\section{8+: sPHENIX Forward Detectors}

- Optimized for high energy

electrons/photons

$-2<n<4$

- e/photon ID

- Hadron PID

- eRHIC ready

- $e+p$

- e+A

- DYvia dielectons @very forward rapidity

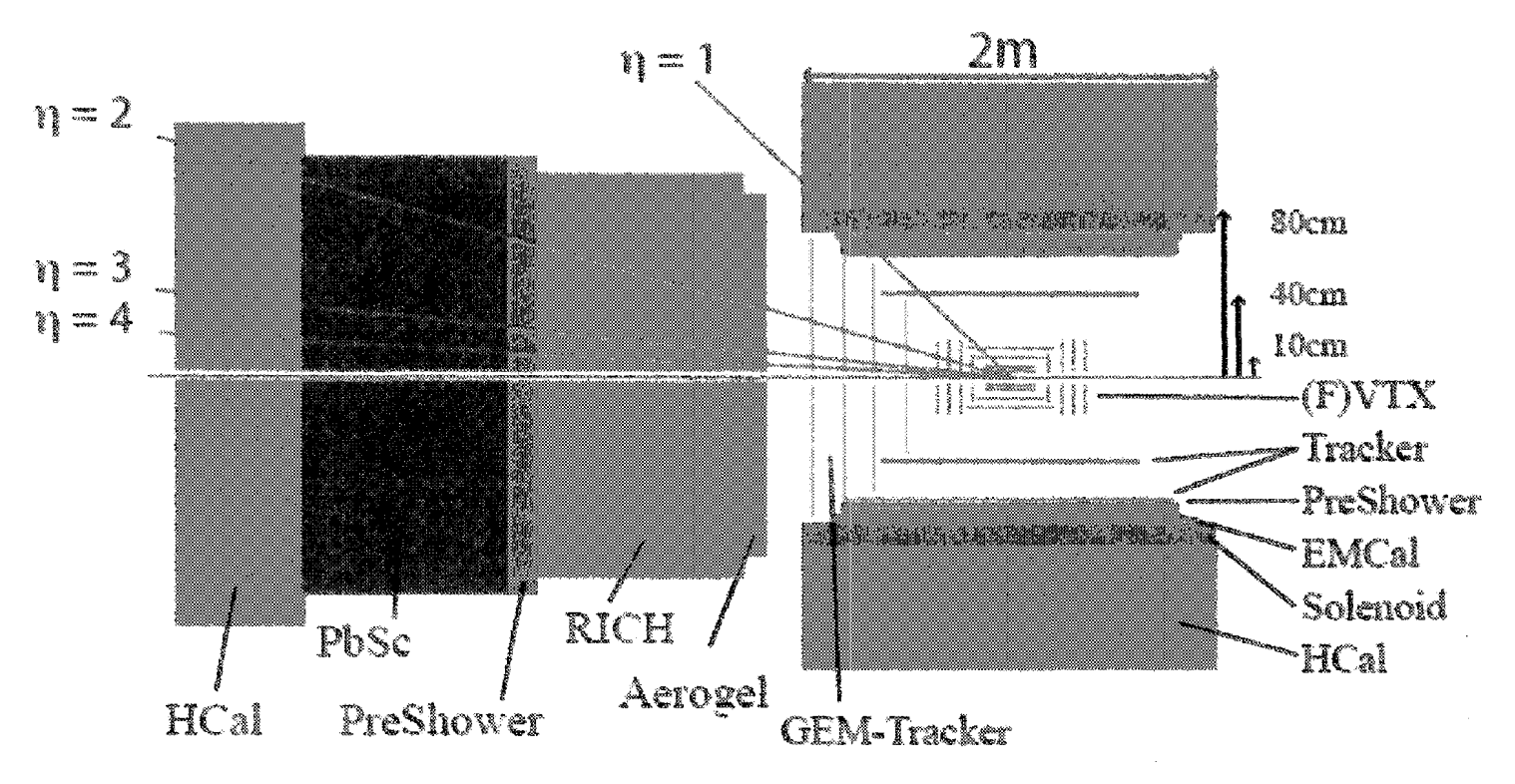

- THACK has 2 momentum nsolution of $\Delta y / y=2 \%$

- RICH Has a dectron efficiency of $\$ 4 \%$ for $p+10 \mathrm{GeV} / 6$

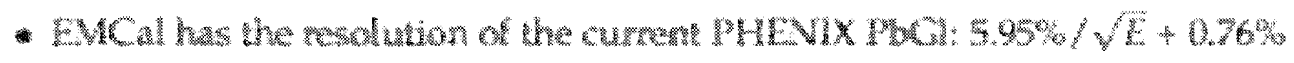

* HCAL has the resolution $50 \% / \sqrt{\mathrm{E}}+5 \%$ (similar $10 \mathrm{CMS}$ ar LHCb) 


\section{Predictions for Drell-Yan process at RHIC}

- Reverse the sign of Sivers from SIDIS and make predictions for DrellYan production at RHIC

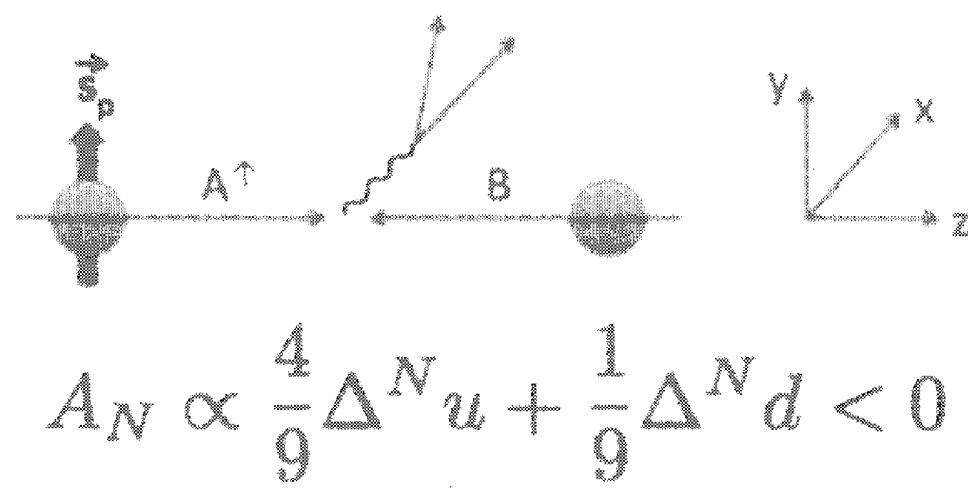

- $A_{N} @$ large rapidities

- PHENIX:

$-1.2<y<2.4$ -dimuons

-sPHENIX:

$-2<y<4$ -dielectrons
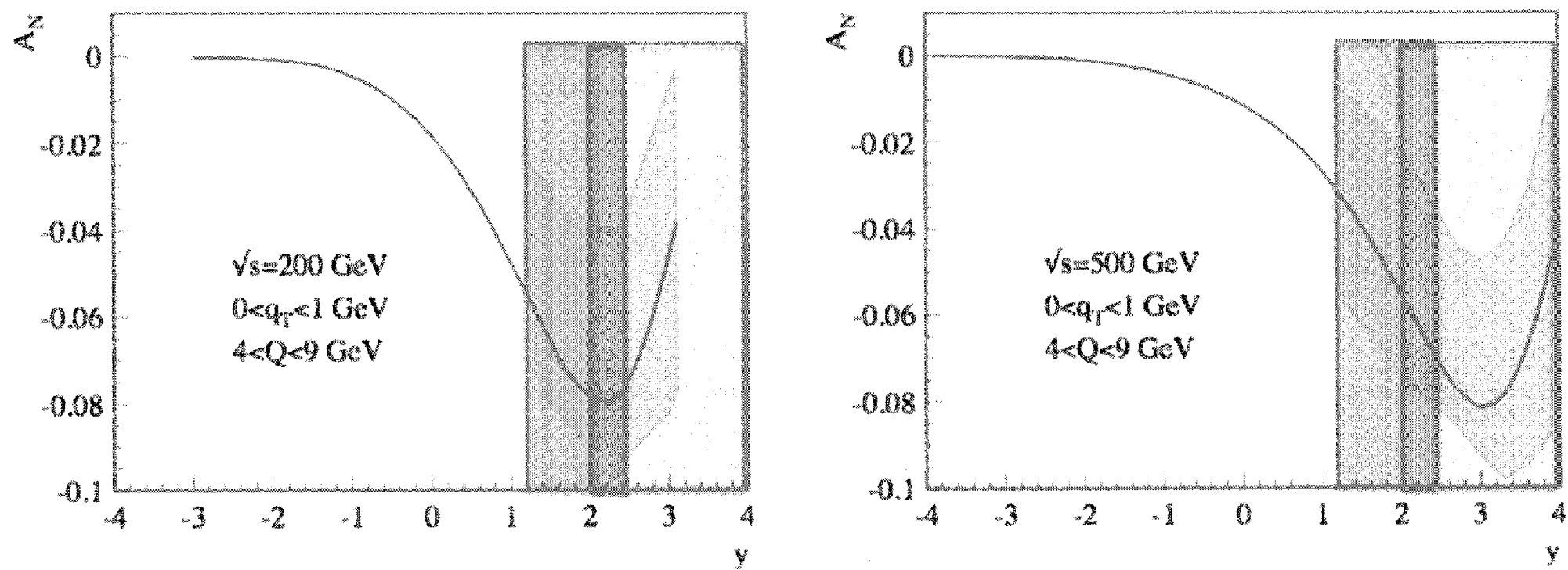


\section{Summary and Outlook}

- Drell-Yan is a powerful tool complimentary to the DIS for exploring parton structures in nucleons and nuclei.

- PHENIXVTX/FVTX upgrades make Drell-Yan measurements possible

- Central arms via electrons

- Forward muon arms

- Some asymmetry measurements (Boer-Mulders alike) possible from upcoming high luminosity longitudinally polarized pp @500GeV

- Possible Test of Drell-Yan A sign change@200GeV and@500GeV after longitudinal $\mathrm{W}$ program, likely after 2017 with forward muon arms

- much improved luminosity needed

- SPHENIX upgrade (2018+)

- Extend the coverage to very forward rapidity $\eta=2 \sim 4$

- Test sign change in Drell-Yan $A_{N}$ via di-electrons at very forward rapidity where significant asymmetry expected

- Explore small-x saturation physics at forward rapidity in p(d)A 


\section{ANDY: Status and Plans \\ L.C. Bland, Brookhaven National Laboratory}

The motivations for a transverse spin Drell-Yan production (DY) measurement were the focus of this workshop. In brief, the objective is to test the robust theoretical prediction that the sign of the Sivers function will differ between semi-inclusive deep inelastic scattering and DY production. Forward production of low-mass virtual photons from the DY process is also of great interest to the study of parton saturation at low- $x$, as discussed at this workshop. This contribution describes a proposal to demonstrate the feasibility of detecting the production of low-mass virtual photons in the forward direction at RHIC.

$A_{N} D Y$ is a proposed experiment at RHIC to measure the analyzing power for forward low-mass Drell-Yan production in transversely polarized proton collisions at $\sqrt{ } \mathrm{s}=500 \mathrm{GeV}$. This presentation reviewed the status of the project and the plans for completing the measurement in the next two years. The basic measurement is to observe the electron and positron decays of a virtual photon produced with $x_{\mathrm{F}}>0.1$ and mass $\mathrm{M}>4 \mathrm{GeV} / \mathrm{c}^{2}$. The energetic dileptons are to be detected in a lead-glass calorimeter (ECal) mounted in the forward direction at RHIC interaction point 2 (IP2). Discrimination of the dilepton signal from background is accomplished by vetoing hadrons using hadronic calorimetry (HCal) mounted immediately behind the ECal. Further hadron/electron discrimination will be made by the use of segmented scintillator sandwiching a converter. The preshower/converter arrangement also will serve to discriminate photon backgrounds from the dielectrons. Simulations show that a left/right symmetric modular ECal and $\mathrm{HCal}$ can be $\sim 30 \%$ efficient for the detection of dielectrons from DY production with $\mathrm{x}_{F}>$ 0.1 and $\mathrm{M}>4 \mathrm{GeV} / \mathrm{c}^{2}$ and can discriminate DY from background. We expect 9400 dielectron events in a $150 \mathrm{pb}^{-1}$ data sample with this modular arrangement. A primary goal of $A_{N} D Y$ is to establish if charge sign discrimination is a requirement for forward dielectron identification for a future forward detector facility at RHIC. Charge sign discrimination in the forward direction is best accomplished using a dipole magnet. A dipole magnet in an interaction region is challenging for a collider.

A primary question addressed during RHIC run 11 is the impact of collisions at IP2 on luminosity and backgrounds at IP6 and IP8. The conclusion from RHIC run 11 is that collisions can be initiated at IP2 without significant impact on IP6 or IP8, and that the integrated luminosity $\left(\mathrm{L}_{\text {int }}\right)$ required for the first transverse spin DY measurement can be delivered in subsequent RHIC runs. Concurrent with the development of IP2 collisions, we recorded $>5 \mathrm{pb}^{-1}$ of polarized proton collisions with left/right symmetric modular HCal detectors. This data is expected to provide results for forward jet analyzing power.

The proposal then is to stage an ECal and the final preshower/converter arrangement for RHIC run 12. We propose to record $150 \mathrm{pb}^{-1}$ in RHIC run 12 for transversely polarized proton collisions at $V_{s}=500 \mathrm{GeV}$ with this apparatus, with the goal of observing J// $\Psi, \Upsilon \rightarrow e^{+} e^{-}$and the dilepton continuum between these two signals as a clear benchmark for DY feasibility. A splitdipole magnet and tracking stations would get staged for RHIC run 13. Our plan is to acquire a second data sample with $\mathrm{L}_{\text {int }}>150 \mathrm{pb}^{-1}$ with tracking through the PHOBOS split-dipole field to quantify the role of charge sign discrimination in suppressing backgrounds. 


\section{$A_{N D Y}$}

\section{"Feasibility Test of Large Rapidity Drell Yan Production at RHIC"}

\section{Letter of Intent submitted 24 May 2010}

http:/Www bnl.gov/npp/docs/pac0610/Cra

PAC presentation:

http/Www.bnl.gov/npp/docs/pac0610/asch

enauer_DY-collider june10.pdf
E.C.Aschenauer, A. Bazilevsky, L.C. Bland, K. Drees, C. Folz, Y. Makdisi, A. Ogawa, P. Pile, T.G. Throwe

Brookhaven National Laboratory

H.J. Crawford, J.M. Engelage, E.G. Judd

University of. California, Berkeley/Space Sciences

$$
\text { Laboratony }
$$

C.W. Perkins

University of. California, Berkeley/Space Sciences

Laboratory/Stony Brook University

A. Derevshchikov, N. Minaev, D. Morozov, L.V. Nogach Institute for High Energy Physics, Protvino

G. Igo, S. Trentalange

University of California, Los Angeles

M. Grosse Perdekamp

University of lllinois

M.X. Liu

Los Alamos National Laboratory

H. Avakian

Thomas Jefferson National Accelerator Facility

E.J.Brash

Christopher Newport University and TJNAF

C.F.Perdrisat

College of Willicm and Mary

V. Punjabi

Norfolk State University

Li, Xuan

Shandong University, China

Mirko Planinic, Goran Simatovic

University of Zagreb, Croatia 


\section{Schematic of detector considered}

Run-12 configuration

(PHOBOS split-dipole expected to be in place, but not used)

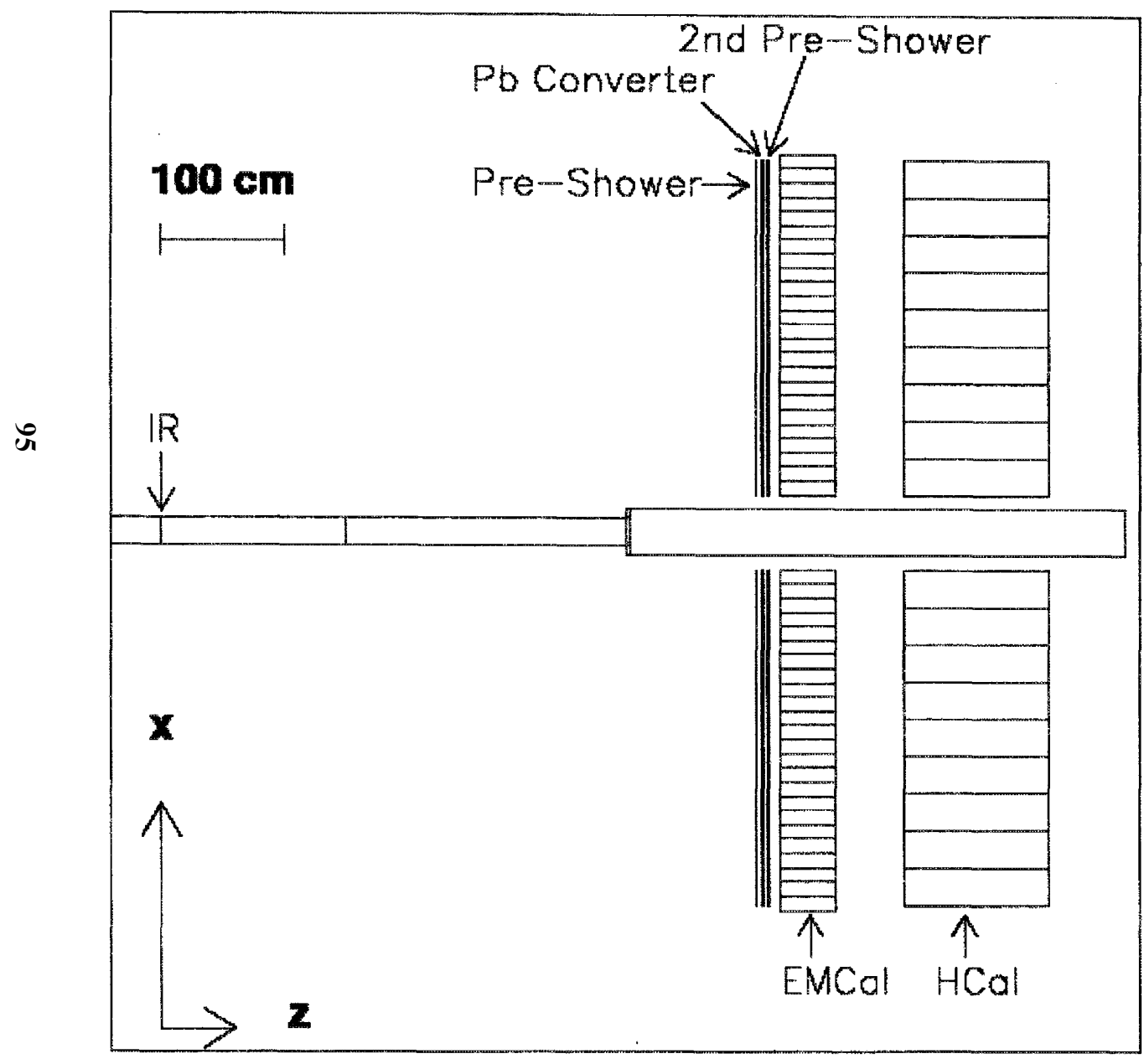

- Hcal is existing $9 \times 12$ modules from E864 (NIM406,227)

- EMcal is modeled as only $(3.8 \mathrm{~cm})^{2} \times(45 \mathrm{~cm})$ lead glass

- Preshower would require construction

http://www.star.bnl.gov/ akio/ip2/topview2.jpeg 


\section{Schematic of detector considered}

Run-13 configuration

(Uses PHOBOS Split Dipole for charge sign)

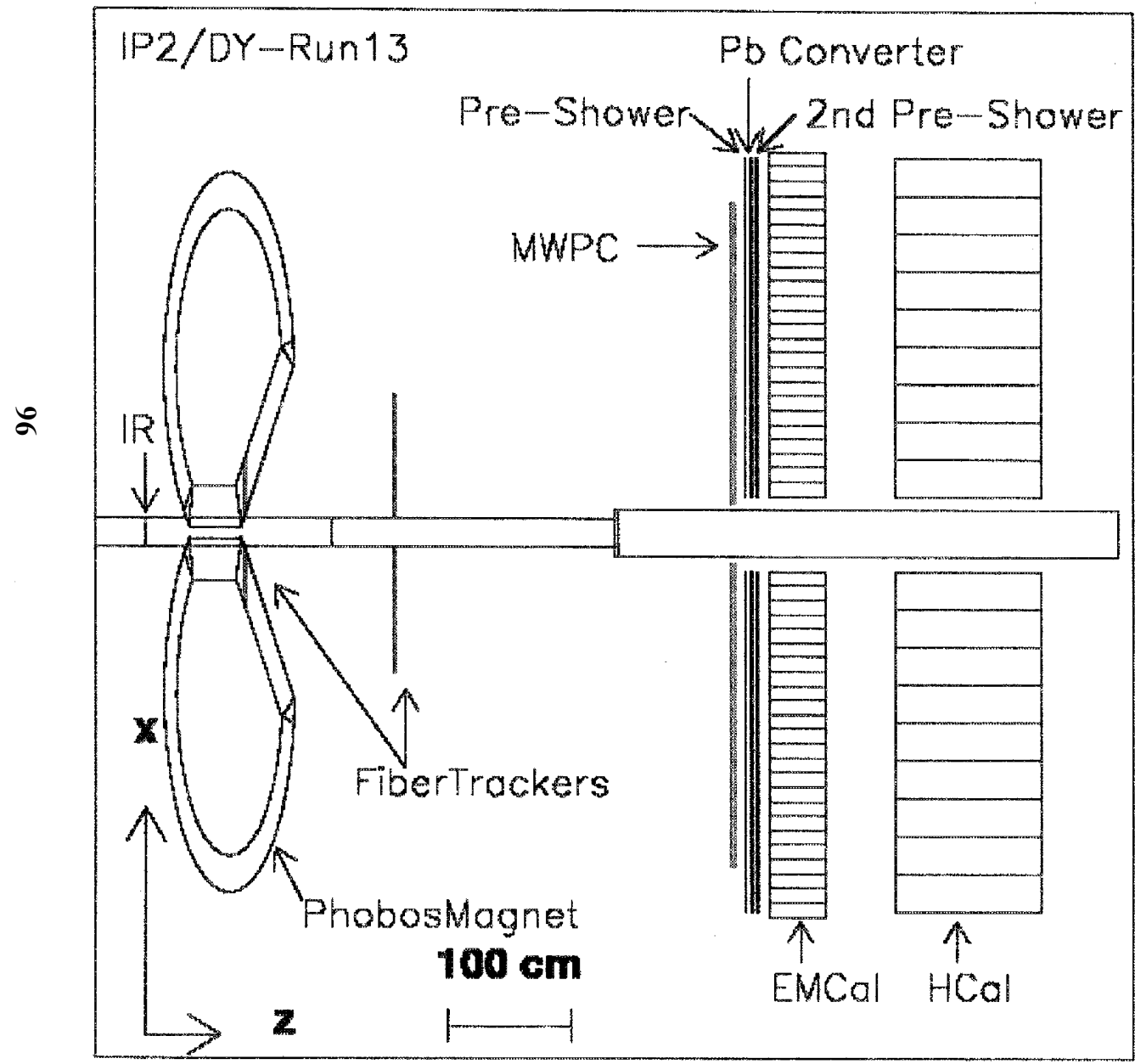

- Hcal is existing $9 \times 12$ modules from E864 (NIM406,227)

- EMcal is modeled as only $(3.8 \mathrm{~cm})^{2} \times(45 \mathrm{~cm})$ lead glass

-Preshower would require construction

- PHOBOS split-dipole magnetic field in GEANT model

- Fiber tracker stations and MWPC require construction

http://www.star.bnl.gov/ akio/ip2/topview_run13.jpeg 


\section{IP2 in January, 2011}

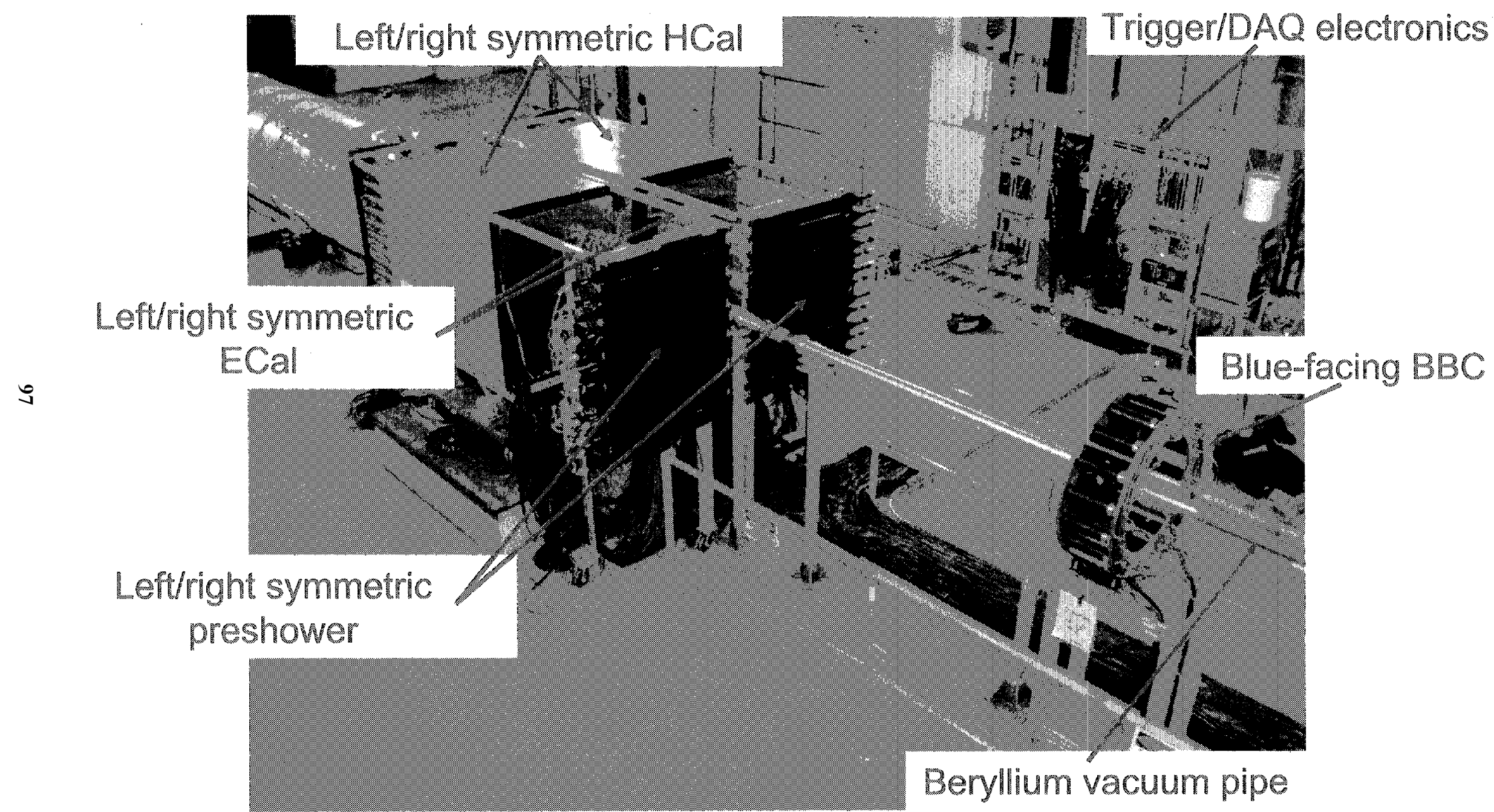




\section{Jet Trigger}

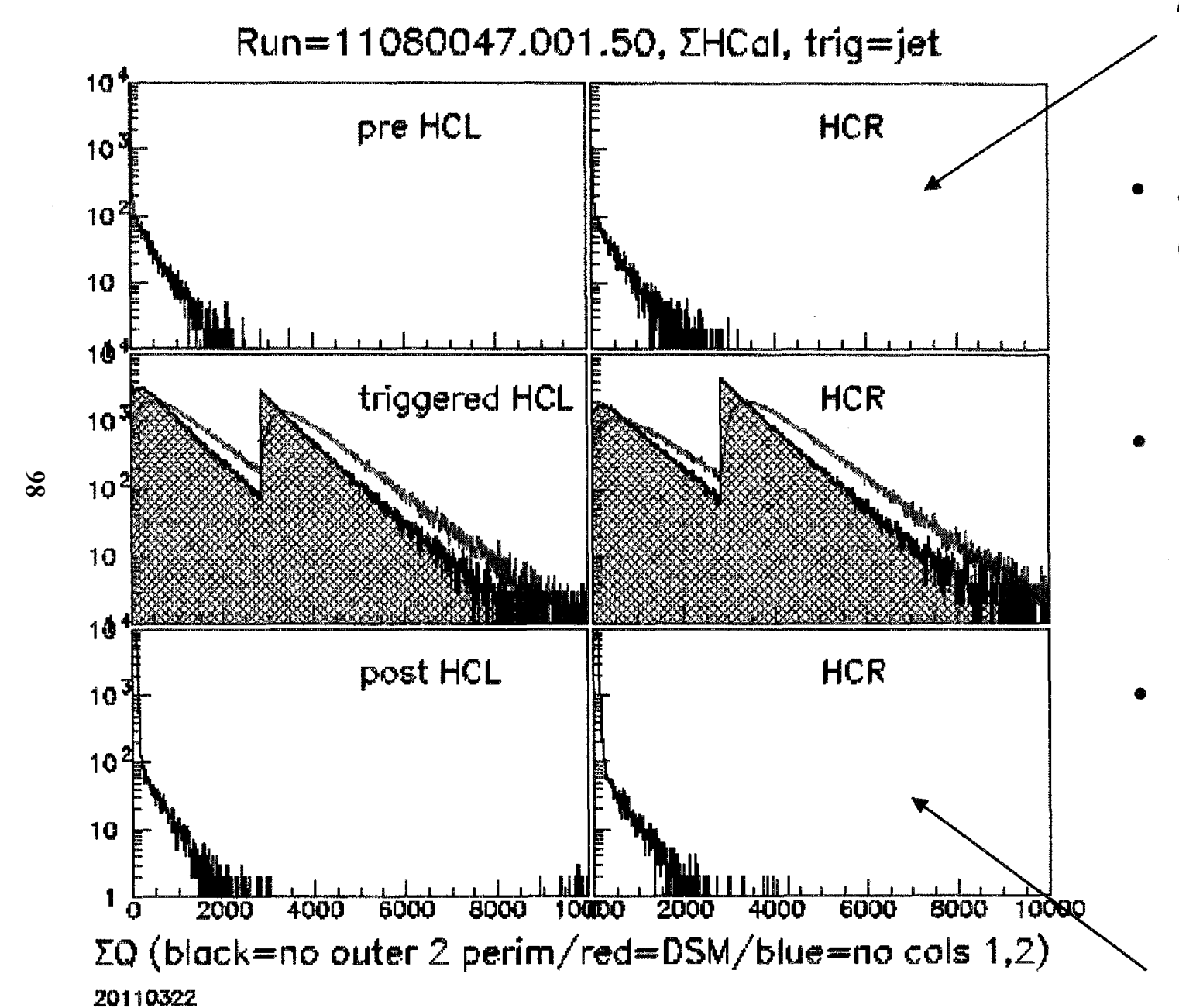

Hadron calorimeter is quiet -107ns before jet event

- Jet trigger sums $\mathrm{HCal}$ response excluding outer two perimeters (rather than just two columns closest to beam)

- Definition is consistent with objective of having jet thrust axis centered in hadron calorimeter modules

- HCal energy scale determination from $\mathrm{ECal} / \mathrm{HCal}$ correlations is underway

Hadron calorimeter is quiet again $\sim 107$ ns after jet event 


\section{RHIC present status and plans}

\section{Wolfram Fischer}

Brookhaven National Laboratory

In Run-11 the peak performance in $250 \mathrm{GeV}$ polarized proton operation has significantly increased for both luminosity and polarization, although at a reduced time in store. The RHIC Run-11 is summarized and the main polarized proton upgrades for the next years are presented. $d-A u$ and $p-A u$ operation, a possible energy upgrade, and ${ }^{3} \mathrm{He}$ operation are discussed. 


\section{Future operation of $A_{n} D Y$}

- Can reduce $\beta^{*}$ at IP2

have run with $\beta^{*}=2.0 \mathrm{~m}$ previously for BRAHMS

$\beta^{*}=1.5 \mathrm{~m}$ probably ok, needs to be tested

- Longer stores

10h instead of $8 \mathrm{~h}$ in Run-11 (depends on luminosity lifetime and store-to-store time)

- Collide earlier in store when conditions are met

needs coordination with polarization measurement, PHENIX and STAR

- Electron lenses (see later) if $A_{n} D Y$ runs beyond Run-13

increases max beam-beam tune spread, currently $\Delta Q_{\text {max,bb }} \approx 0.015$ can be used for to increase $\xi \sim N_{b} / \varepsilon$ and/or number of collisions

Run-11 luminosity at $A_{n} D Y$ : max $\sim 0.3 \mathrm{pb}^{-1} /$ store

With improvements:

$\sim 3 x$ increase,

$\sim 10 \mathrm{pb}^{-1 /}$ week

[all preliminary]

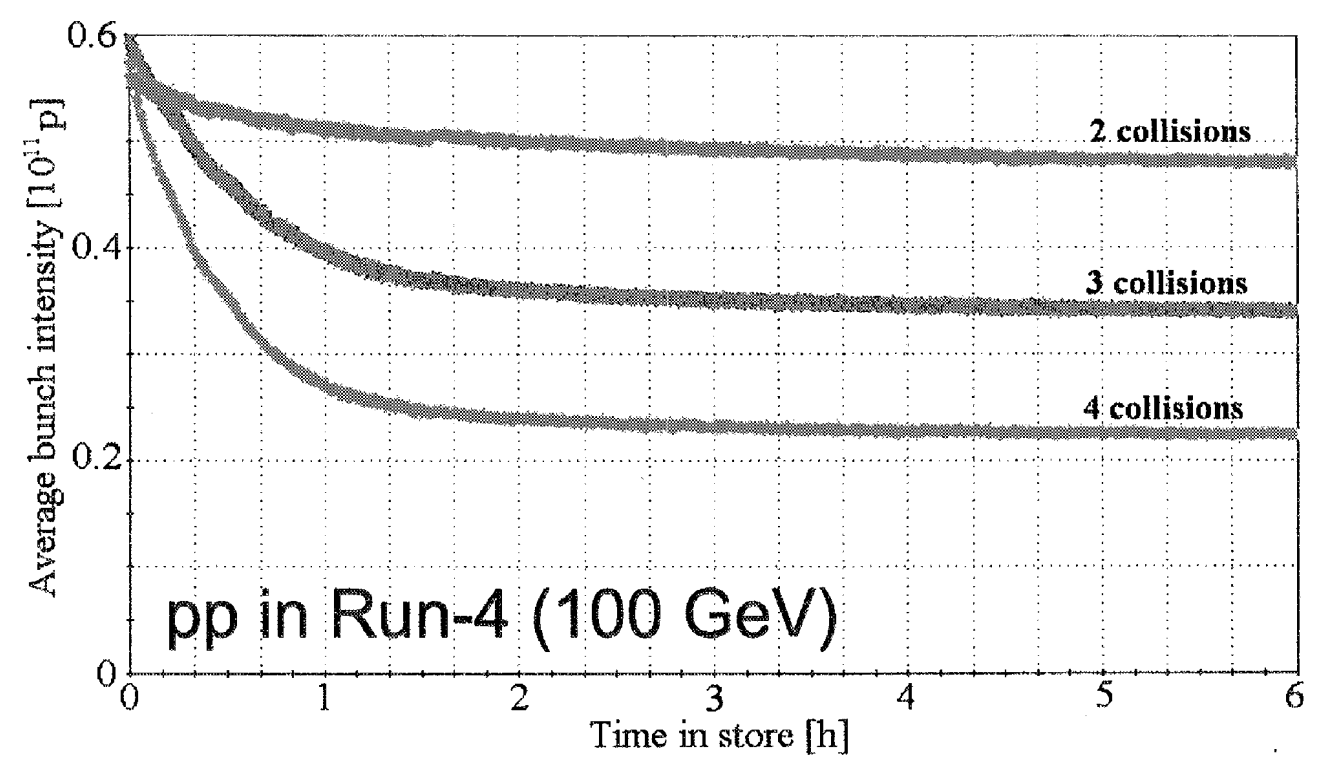




\section{Asymmetric collisions ( $p-A u)$}

- $p-A u$ was considered in RHIC design (D. Trbojevic), no operation yet

100.8 GeV p on 100.0 GeV/nucleon Au $\left(\gamma_{p}=\gamma_{A U}=107.4\right)$

- Need to translate DX magnets horizontally by $4.33 \mathrm{~cm}$

$p$ are bent stronger than $\mathrm{Au}^{79+}$

- For energy scan need to match Lorentz factor $\gamma$ of both beams

\section{Parameter}

\section{unit}

No of bunches

lonsbunch, initial

Average beam currenting

Stored energy per beam

$p$ *

Hour glass factor

Beam-beam parameter $\xi / 1 P$

Peak luminosity

Average / peak luminosity

Average store luminosity

Time in store

Maximum luminosity/week

Minimum luminosity/week

unit
$\ldots$
$10^{2}$
$\mathrm{~mA}$
$\mathrm{MJ}$
$\mathrm{m}$
$\cdots$
$10^{3}$
$10^{28} \mathrm{~cm}^{-2} \mathrm{~s}^{13}$
$\%$
$10^{2 \%} \mathrm{~cm}^{2 /} \mathrm{s}^{4}$
$\%$
$\mathrm{nb}^{1}$
$\mathrm{nb}^{1}$

\begin{tabular}{c}
$\mathbf{p - A u}$ \\
111 \\
100 \\
139 \\
0.85 \\
1.00 \\
4.3 \\
30 \\
60 \\
18 \\
55 \\
60 \\
\hline
\end{tabular}

$\mathrm{p}-\mathrm{Au}$

Wolfram Fischer 


\section{Energy upgrade - W. MacKay, C-A/AP/422}

observed quenches in arc dipoles

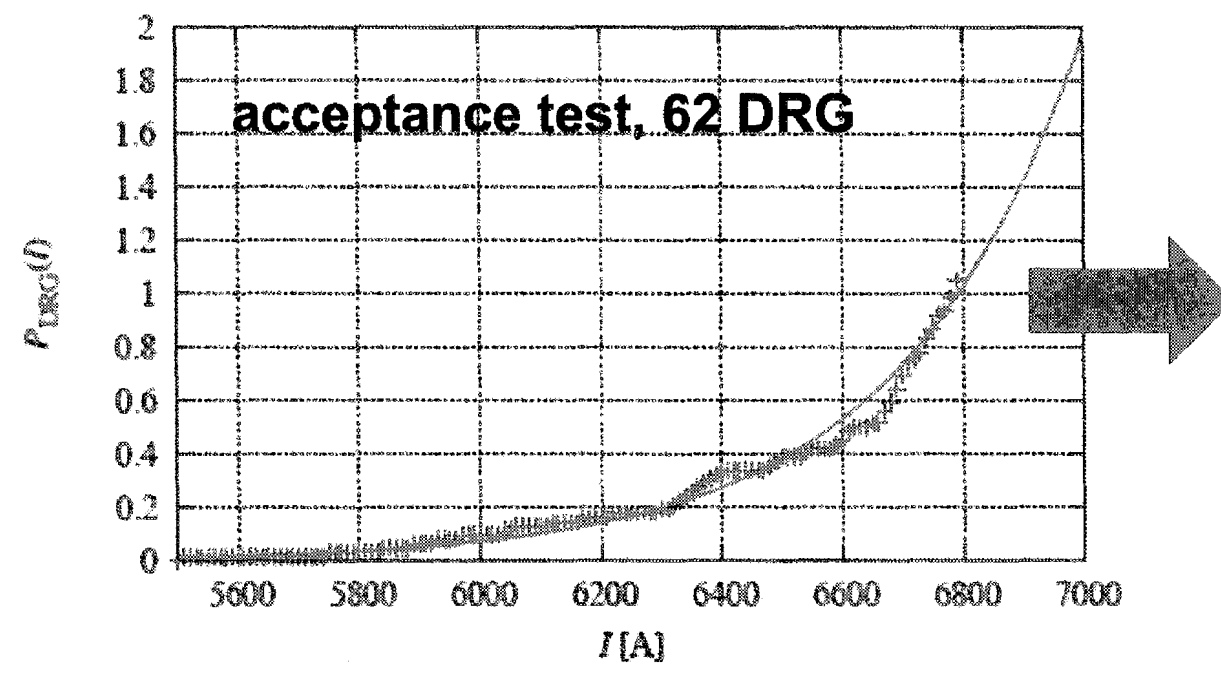

estimated \# of training quenches

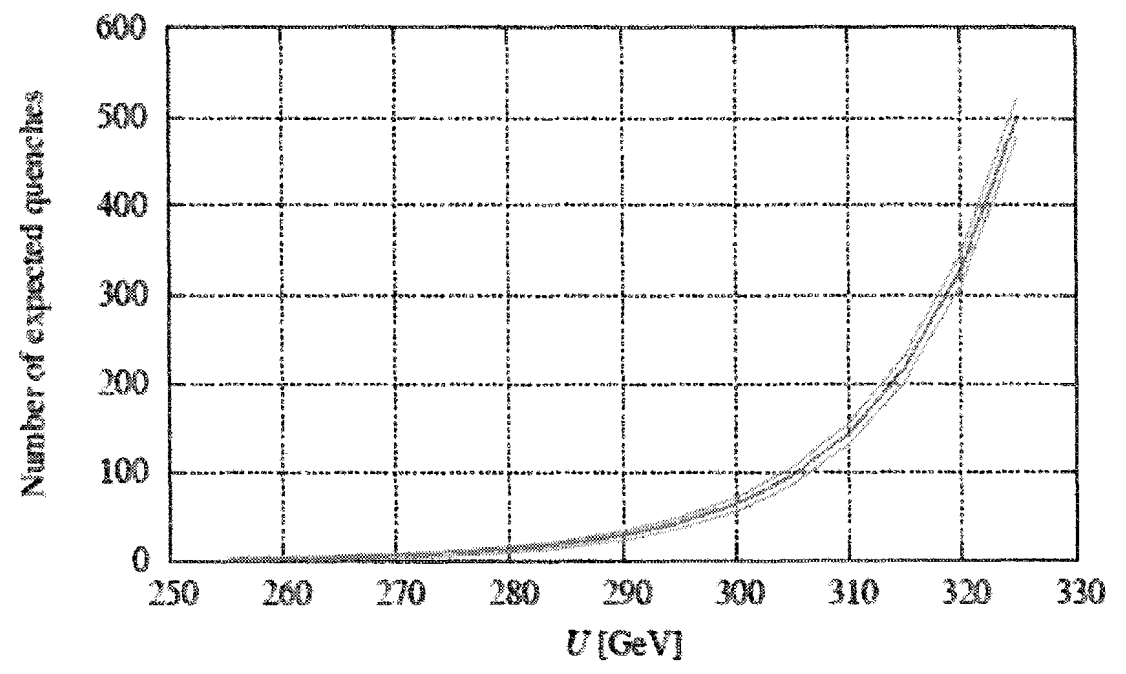

Conclusion:

- $10 \%$ increase to $275 \mathrm{GeV}\left(+45 \%\right.$ in $\left.\sigma_{\mathrm{w}}\right)$ feasible with current magnets about $20 \mathrm{DX}, 10$ other training quenches, more cooling at some current leads

- Requires some hardware upgrades (dump kicker, power supplies)

- Effect on polarization still needs study

- Energies $>275 \mathrm{GeV}$ require too many training quenches

hundreds of arc dipole training quenches alone for $325 \mathrm{GeV}$ 


\section{Polarized ${ }^{3} \mathrm{He}$}

[Summary W. MacKay, CAD MAC-05, 09/15/2010]

of Deuterons not good in RHIC - perhaps in a figure-8 ring.

n $\mathrm{He}^{3}$ looks promising: no real show stoppers.

- Source: ${ }^{3} \mathrm{He}^{+2}$ OPPIS source - proposal: Miner/Zelenski See Anatoli Zelenski's presentation.

- $|G \gamma|_{\max }$ is higher for $\mathrm{He}^{3}$ :

- More and Stronger resonances in all rings.

- ${ }^{3}$ He polarimeters need to be developed.

- AGS cold snake may be sufficient at lower field. AGS warm snake (fixed field) might be too strong ( 14\%).

- AGS injection and extraction spin-matching: not too bad.

- Booster to AGS may need matching (depends on AGS snakes).

- RHIC snakes and rotators will work with lower fields.

- Lower injection rigidity for RHIC should be OK.

- Injection orbit excursions reduced. 


\section{Summary - RHIC performance}

- Run-11 $\mathrm{p}^{\wedge} \mathrm{p}^{\wedge}$ results:

$P>46 \%, L_{\text {peak }}=150 \times 10^{30} \mathrm{~cm}^{-2} \mathrm{~s}^{-1}, L_{\text {avg }}=85 \times 10^{30} \mathrm{~cm}^{-2} \mathrm{~s}^{-1}$

(all new records for peak performance, and all within Run-11 projections)

Integrated luminosity below expectation due to down time

$A_{n} D Y$ tested, ran with relatively small impact on STAR/PHENIX

- Main hardware upgrades for $p^{\wedge} p^{\wedge}$ (commissioning planned for Run-13)

Polarized source: $P+5 \%$, intensity +order of magnitude

Electron lenses : up to $2 x$ more luminosity with source upgrade

- Asymmetric collisions (d-Au and p-Au)

Expect up 2x more luminosity for future d-Au operation rel. to Run-8

p-Au possible with change of DX location $\left(\gamma_{p}=\gamma_{A u}=107.4\right)$

- Limited energy upgrade possible, $10 \%$ to $275 \mathrm{GeV}$ protons

Effect on polarization still needs study, requires hardware upgrades

- Polarized ${ }^{3} \mathrm{He}\left(p-{ }^{3} \mathrm{He},{ }^{3} \mathrm{He}-{ }^{3} \mathrm{He}\right)$

Polarized ${ }^{3} \mathrm{He}$ source R\&D has started (with MIT, using EBIS)

Acceleration and storage in RHIC should be possible

${ }^{3} \mathrm{He}$ polarimetry at high (esp. absolute) needs R\&D 


\title{
Theoretical perspectives on Drell-Yan production measurements
}

\author{
Jian-Wei Qin \\ Physics Department, Brookhaven National Laboratory, Upton, NY 11973, USA
}

I noticed that almost all talks in this workshop addresses the "theoretical perspectives on DrellYan production measurements". In this talk, I will list a number of opportunities and challenges associated with Drell-Yan production measurements, and try not to repeat too much what other people have said.

By measuring two leptons, the Drell-Yan process is a hard probe with two natural scales: invariant mass $Q=\sqrt{q^{2}}$ and total transverse momentum $q_{\perp}$ of the pair. By measuring Drell-Yan lepton pair at different combinations of these two momentum scales, the measurement can provide rich information on QCD dynamics and colliding hadron's partonic structure. For example, when $q_{\perp} \ll Q$, the transverse momentum dependent (TMD) factorization formalism should work for the Drell-Yan cross section, and the measurement of Drell-Yan lepton pairs in this kinematic regime probes the TMD parton distributions and dynamics of partons' transverse motion inside a colliding hadron. On the other hand, when $q_{\perp} \sim Q$ or $q_{\perp} \gg Q$, the collinear factorization formalism should work better, and the measurement should provide clean information on collinear parton distributions, in particular, the gluon distributions. Exploring the rapidity dependence of the lepton pair can help probe parton densities at very small parton momentum fractions, in particular, in the region where $Q_{s} \sim q_{\perp}$. Furthermore, by measuring the angular distribution of the lepton pair in the pair's rest frame, Drell-Yan measurement provides excellent information on the quantum interference of different spin states of the vector boson that decays into the lepton pair.

One of the most important predictions of TMD factorization formalism is the sign change of the Sivers function and the Boer-Mulder function between the SIDIS and Drell-Yan measurement. The sign change is the immediate consequence of the TMD factorization, and the parity and the time-reversal invariance of strong interaction. The test of the sign change is clearly a critical test of the TMD factorization formalism. However, one has to compare the distributions from SIDIS and Drell-Yan at the same momentum fraction $x$ and parton transverse momentum $k_{\perp}$ in order to have a true test of the sign change. This is because the sign of the spin asymmetries could be different at the different effective value of $x$ or $k_{\perp}$ if there is a node in either the $x$-dependence or $k_{\perp}$ dependence of the TMD distributions.

To test spin asymmetries, it is very important to understand both the numerator and the denominator. The denomenator - the spin averaged Drell-Yan cross section at low $q_{\perp}$ and large $Q$ requires QCD resummation of large logarithms. For the same kinematics, the resummation is also needed for the numerator - the spin dependent cross section. Theory difficulties exist in controlling lepton angular distributions at low $q_{\perp}$. If one describes the low $k_{\perp}$ behavior in terms of TMD parton distributions, it is critical to understand the $Q^{2}$ dependence of TMD distributions, which is still lacking.

Test of the predicted strong suppression of Drell-Yan production in the very forward region of $d A$ collisions is exciting. Quantitative comparison between various theory calculations is needed. Verify the predicted sign change of the power correction in low $Q$ region between inclusive Drell-Yan and DIS should be very interesting too. In conclusion, Drell-Yan lepton pair production is one of the oldest hard process proposed to test QCD, and it is still a very good one! 


\section{Excellent probe of gluon distribution}

Nuclear modification factor:

Kang, Qun, Voghisang, pro 2008

$$
R_{\text {dAu }} \equiv \frac{1}{\left\langle N_{\text {coll }}\right\rangle} \frac{d^{2} N^{d A^{2}} / d Q_{T} d y}{d^{2} N p p / d Q_{T} d y} \stackrel{\text { min.bins }}{=} \frac{\frac{1}{2 A} d^{2} \sigma^{d A n} / d Q_{T} d y}{d^{2} \sigma^{p p} / d Q_{T} d y}
$$

RHIC kinematics - if dominated by single scattering:
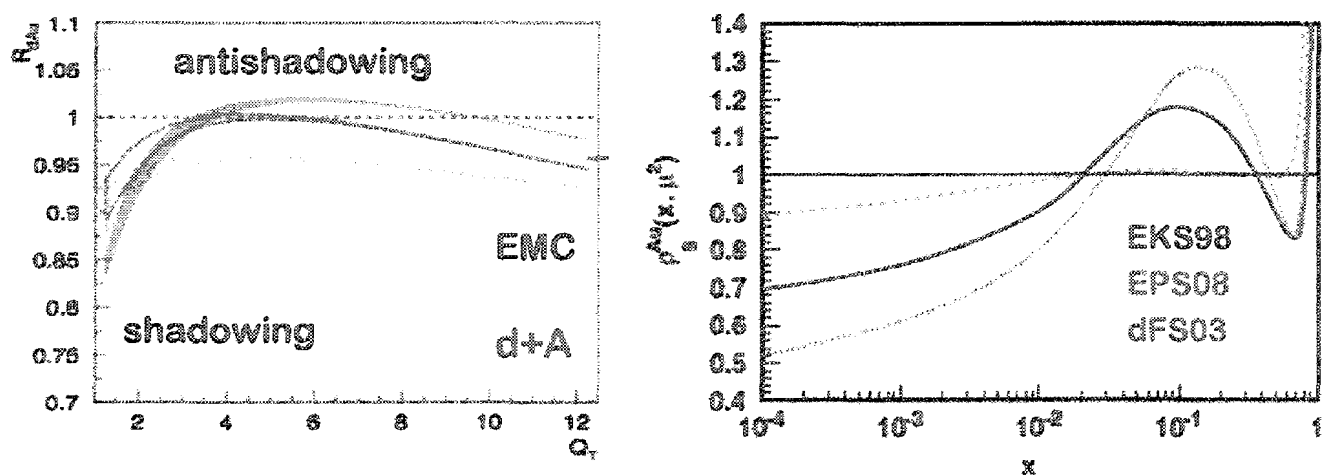

- The band is given by $k=1$ (top lines) and $k=0$ (bottom lines)

- Ratio follows the feature of gluon distribution if turns off isospin

- No suppression if removing isospin effect

\section{Saturation and CGC physics}

Forward rapidity $(y>0)$ :

If $\mathrm{Q}_{T} \propto \mathrm{Q}_{\mathrm{S}}$, collinear factorization fails

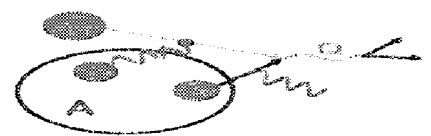

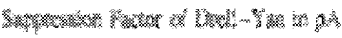

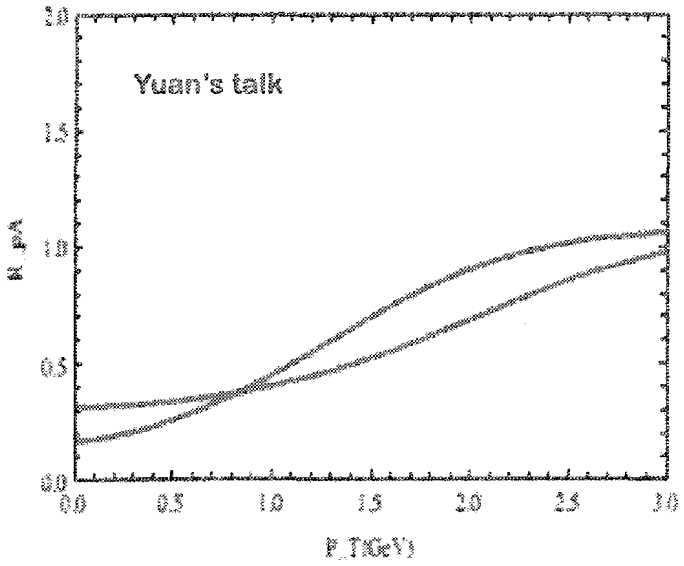

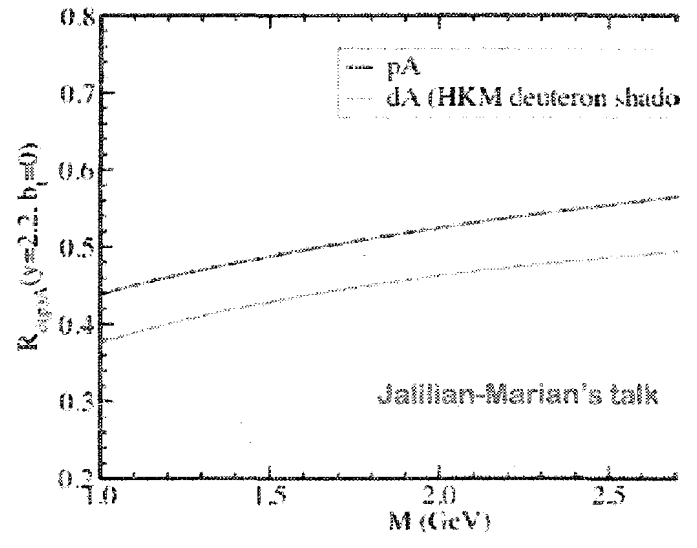

Nuclear shadowing cannot produce such suppression!

Theory challenge: Role of $p_{T}$ ? 


\section{Another sign change}

U Power correction to DIS - single scale:

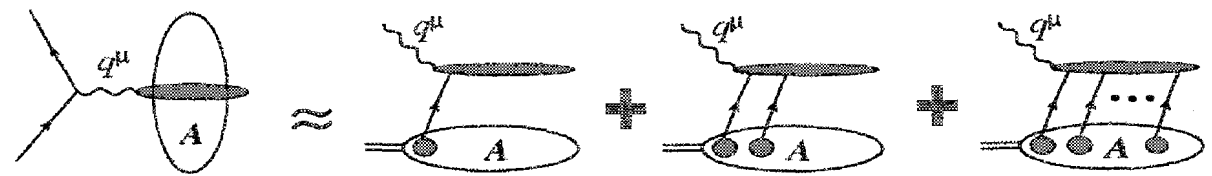

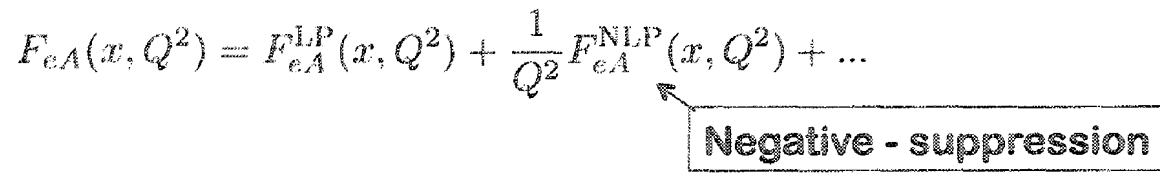

D Power correction to inclusive DY - single scale:

$$
\frac{d \sigma_{p A}}{d Q^{2}}=\frac{d \sigma_{p H}^{L P}}{d Q^{2}}+\frac{1}{Q^{2}} \frac{d \sigma_{p A}^{N L^{p}}}{d Q^{2}}+\ldots
$$

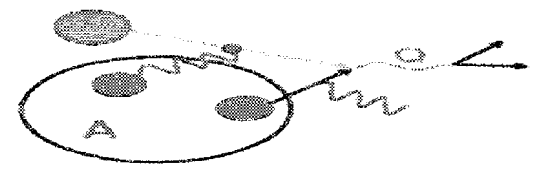

$$
\text { Compton gives negative contribution in CO factorization }
$$

\section{Summary and outlook}

$\square$ Drell-Yan process is one of the oldest hard process proposed to test QCD - it still a very good one!

DThe proof of QCD factorization for Drell-Yan is solid (LP + NLP for collinear, LP for TMD)

$\square$ The test of the sign change of the Sivers function is a critical test of TMD factorization!

$\square$ Drell-Yan could provide much more than the sign change of Sivers function

Thank you! 


\section{TMD fracture functions in SIDIS and DY}

\section{Aram Kotzinian}

Torino University and INFN, Italy and YerPhI, Armenia

The Fracture Function formalism was introduced by Trentadue and Veneziano in 1994 to describe hadron production in the target fragmentation region (TFR) of SIDIS in collinear configuration.

Recently we generalized this formalism for the spin and transverse momentum dependent fracture functions (see M.Anselmino, V.Barone and A.K., arXiv:1102.4214; PLB 699 (2011) 108). In total 16 LO fracture functions are needed to describe spinless hadron production. One particle production in the TFR of polarized SIDIS gives access only to $4 \mathrm{k}_{\mathrm{T}}$-integrated fracture functions.

To study other fracture functions one need to "measure" scattered quark transverse polarization. Collins effect for hadron produced in the current fragmentation region (CFR) allows to access these functions via azimuthal asymmetries measurements in double hadron (one in CFR, another in TFR) production (DSIDIS) process.

Another way to study these fracture functions is to measure the azimuthal asymmetries in the polarized semi-inclusive DY (SIDY) processes when together with high mass lepton pair one spinless hadron is also detected.

The expression for the LO cross sections in polarized DSIDIS and SIDY processes are presented. 


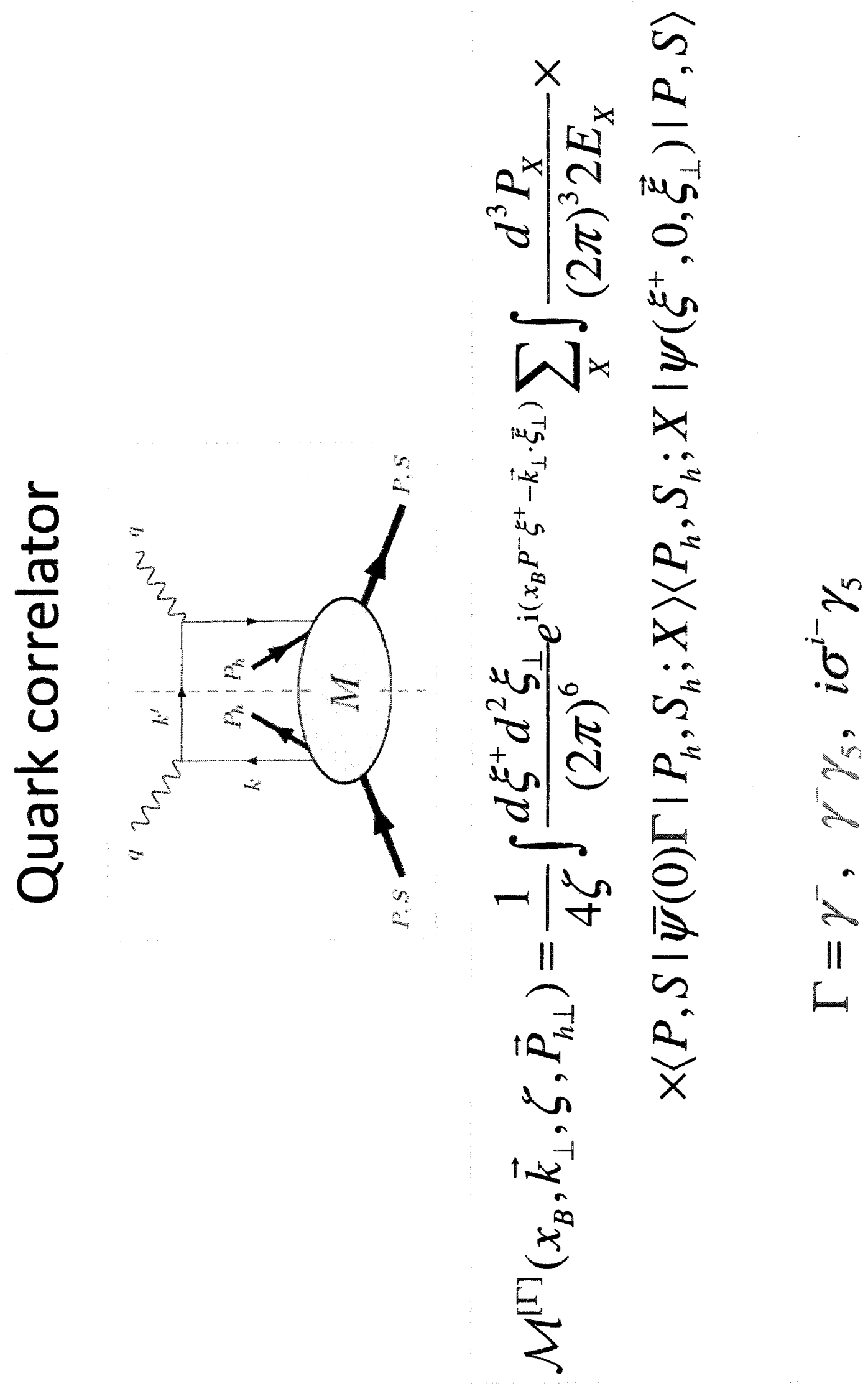


STMD Fracture Functions for spinless hadron production

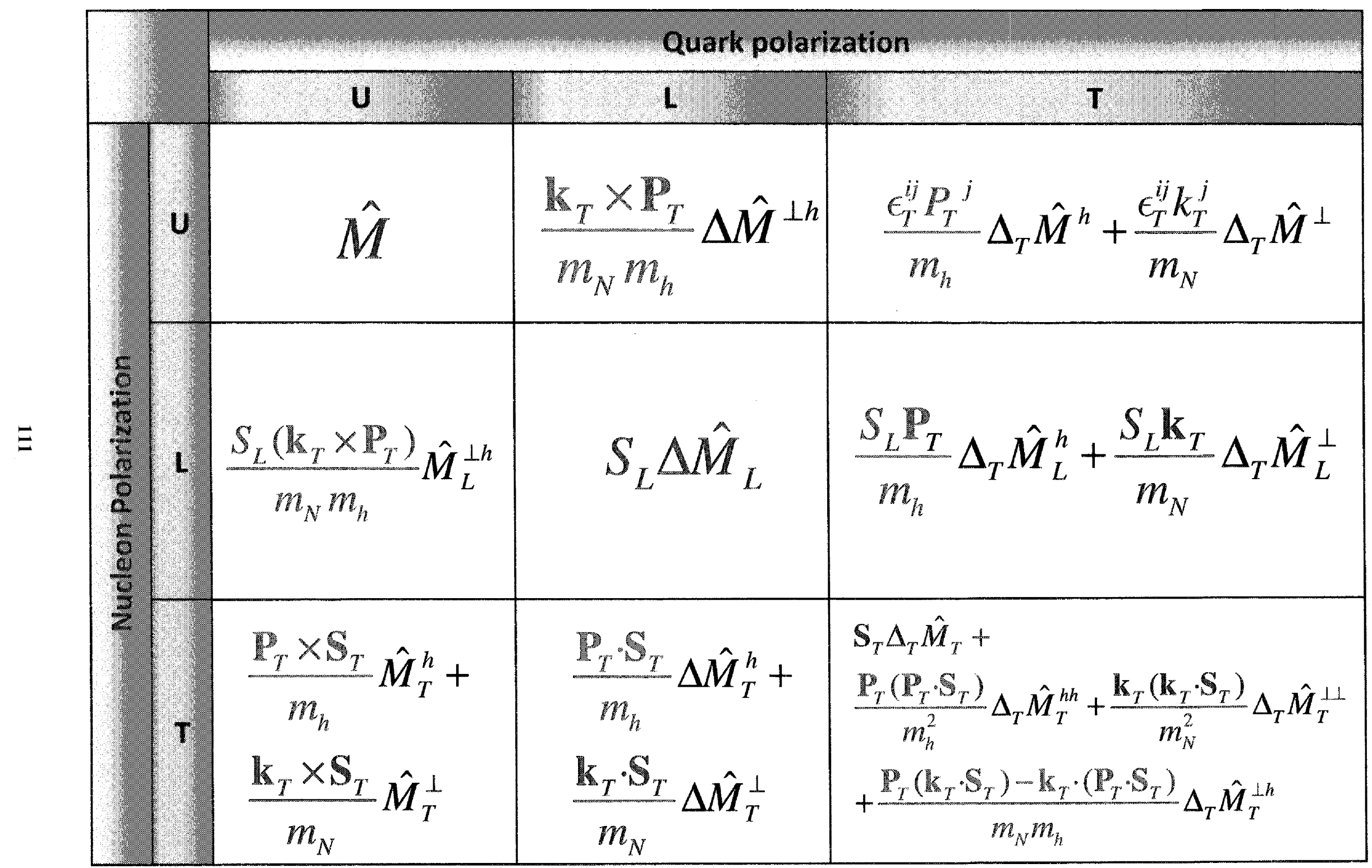




\section{LO cross-section in TFR}

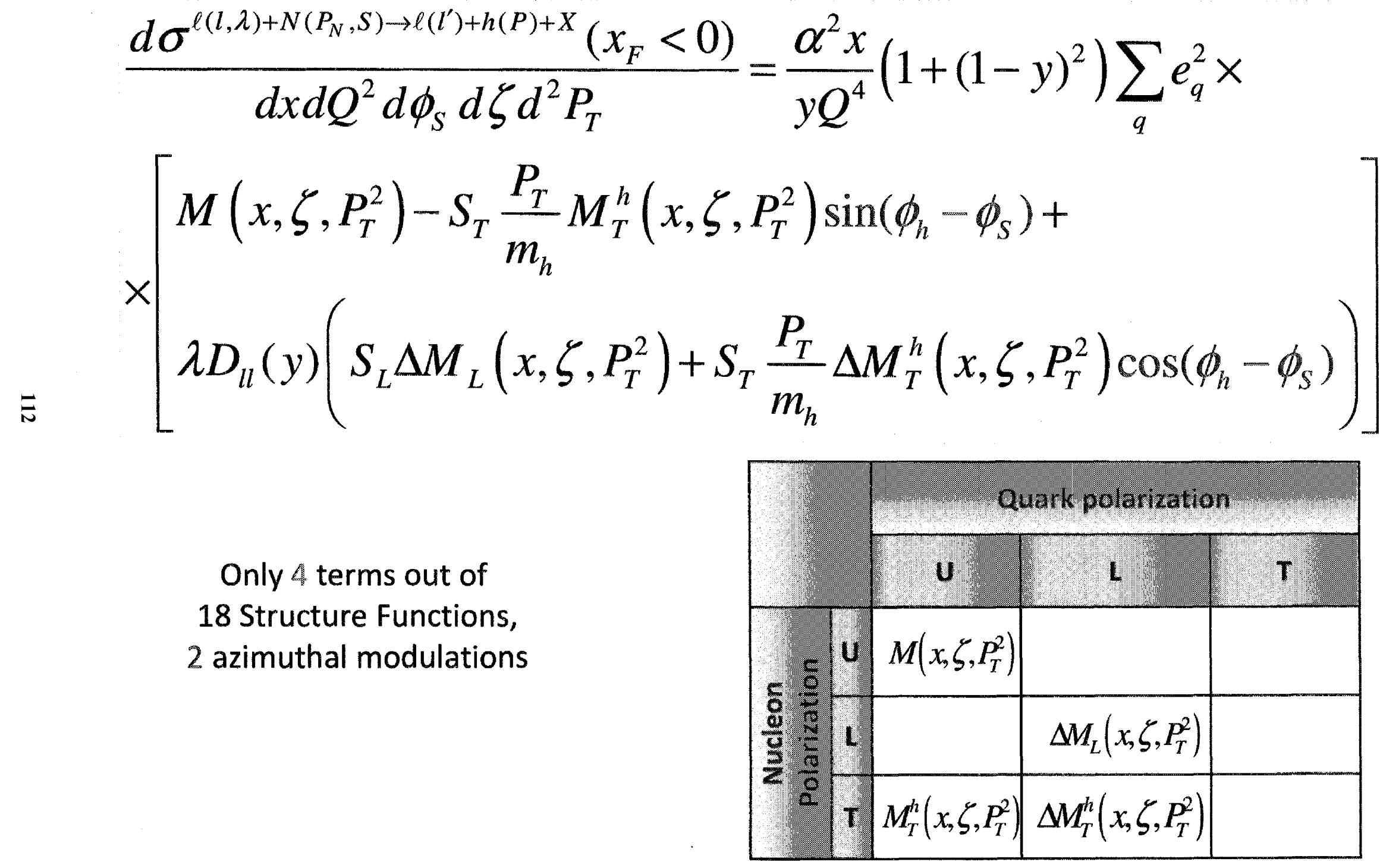




\section{SIDY cross section}

$$
\begin{aligned}
\frac{d \sigma}{d^{4} q d \Omega d \zeta d^{2} P_{T}}= & \frac{\alpha_{e m}^{2} x_{a} x_{b}}{2 q^{4}} \frac{1}{N_{c}} \sum_{q} e_{q}^{2} \int d^{2} \vec{k}_{a T} d^{2} \vec{k}_{b T} \delta^{(2)}\left(\vec{q}_{T}-\vec{k}_{a T}-\vec{k}_{b T}\right) \times \\
& \times\left(\begin{array}{l}
\left(1+\cos ^{2} \theta\right)\left(\Phi^{q\left[\gamma^{+}\right]} \overline{\mathcal{M}}^{q\left[\gamma^{-}\right]}+\Phi^{q\left[\gamma^{+} \gamma_{5}\right]} \overline{\mathcal{M}}^{q\left[\gamma^{-} \gamma_{s}\right]}\right) \\
+\sin ^{2} \theta\left(\begin{array}{l}
\cos 2 \phi\left(\delta^{i 1} \delta^{j 1}-\delta^{i 2} \delta^{j 2}\right) \\
+\sin 2 \phi\left(\delta^{i 1} \delta^{j 2}+\delta^{i 2} \delta^{j 1}\right)
\end{array}\right) \Phi^{q\left[i \sigma^{\left.j^{i} \gamma_{s}\right]}\right]} \overline{\mathcal{M}}^{q\left[i \sigma^{\left.j-\gamma_{5}\right]}\right.} \\
+\{\Phi \leftrightarrow \bar{\Phi}, \overline{\mathcal{M}} \leftrightarrow \mathcal{M}\}+\mathcal{O}(1 / q)
\end{array}\right) \\
= & \frac{\alpha_{e m}^{2} x_{a} x_{b}}{2 q^{4}}\left(\begin{array}{l}
\sigma_{U U}+S_{b L} \sigma_{U L}+S_{b T} \sigma_{U T} \\
+S_{a L} \sigma_{L U}+S_{a L} S_{b L} \sigma_{L L}+S_{a L} S_{b T} \sigma_{L T} \\
+S_{a T} \sigma_{T U}+S_{a T} S_{b L} \sigma_{T L}+S_{a T} S_{b T} \sigma_{T T}
\end{array}\right)
\end{aligned}
$$




\section{CONCLUSIONS}

- New members appeared in the polarized TMDs family -- 16 LO STMD fracture functions

- For hadron produced in the TFR, only $4 k_{T}$-integrated fracture functions of unpolarized and longitudinally polarized quarks are probed.

- SSA contains only a Sivers-type modulation $\sin \left(\phi_{h}-\phi_{S}\right)$ but no Collins-type $\sin \left(\phi_{h}+\phi_{S}\right)$ or $\sin \left(3 \phi_{h}-\phi_{s}\right)$. The eventual observation of Collins-type asymmetry will indicate that LO factorized approach fails and long range correlations between the struck quark polarization and $\mathrm{P}_{\mathrm{T}}$ of produced in TFR hadron might be important.

- DSIDIS cross section at LO contains 2 azimuthal independent and 20 azimuthally modulated terms.

- SIDY cross section at LO contains 2 azimuthal independent, 20 lepton azimuth independent and 52 lepton azimuth dependent terms

- The ideal place to test the fracture functions factorization and measure these new nonperturbative objects are JLab12 and EIC facilities with full coverage of phase space and polarized SIDY

- To do

- Factorization proof (SIDIS, DSIDIS, SIDY).

- Structure of Wilson lines. SIDIS $\leftrightarrow$ DY universality: sign changes of some fracture functions? Higher twist. Polarized hadron production. Phenomenology: parameterizations, simple models. Other processes: $\mathrm{P} \uparrow+\mathrm{P} \rightarrow \pi+\mathrm{X}$, $\mathrm{P} \uparrow+\mathrm{P} \rightarrow \pi+\mathrm{jet}+\mathrm{X}, \ldots$ 
What the Drell-Yan measurement can offer us at small- $x$ ?

May 12, 2011

PENASTATE 


\section{A Tale of Two Gluon Distributions}

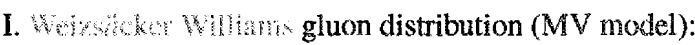

$$
\begin{aligned}
x G^{(1)} & =\frac{S_{\perp}}{\pi^{2} \alpha_{s}} \frac{N_{c}^{2}-1}{N_{c}} \\
& \times \int \frac{d^{2} r_{\perp}}{(2 \pi)^{2}} \frac{e^{-i k_{\perp} \cdot r_{\perp}}}{r_{\perp}^{2}}\left(1-e^{-\frac{r_{\perp}^{2} Q_{s}^{2}}{2}}\right)
\end{aligned}
$$

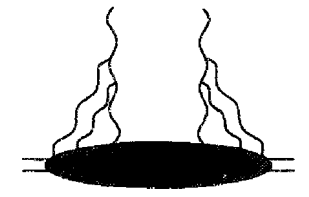

II. Cotor Drase gluon distributions:

$x G^{(2)}=\frac{S_{\perp} N_{c}}{2 \pi^{2} \alpha_{s}}$

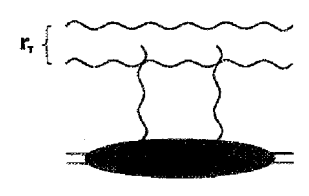

$\times \int \frac{d^{2} r_{\perp}}{(2 \pi)^{2}} e^{-i k_{\perp} \cdot r_{\perp}} \nabla_{r_{\perp}}^{2} N\left(r_{\perp}\right)$

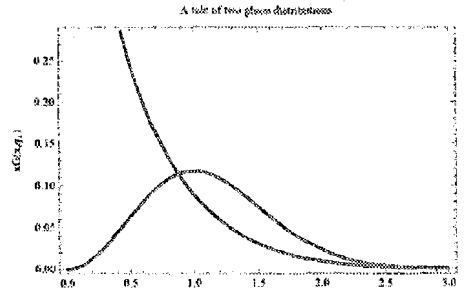

PENNSTATE

4 


\section{$\gamma+$ Jet in pA collisions}

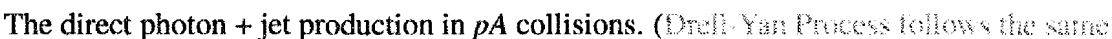

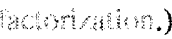

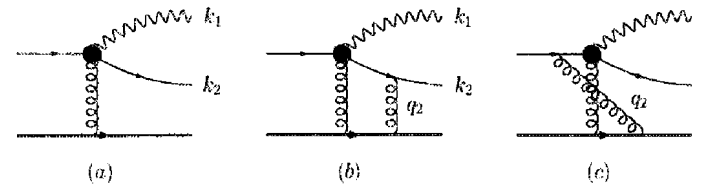

Wroto motat antwath:

$$
\begin{array}{ll}
\frac{d \sigma_{\mathrm{DP}}^{\mathrm{pA} \rightarrow \gamma^{*} q+X}}{d y_{1} d y_{2} d^{2} k_{1 \perp} d^{2} k_{2 \perp} d^{2} b}= & \sum_{f} x_{p} q_{f}\left(x_{p}, \mu\right) \frac{\alpha_{e, m} \cdot e_{f}^{2}}{2 \pi^{2}}(1-z) F_{x_{g}}\left(q_{\perp}\right) \\
& \times\left\{\left[1+(1-z)^{2}\right] \frac{z^{2} q_{\perp}^{2}}{\left[\tilde{P}_{\perp}^{2}+\epsilon_{M}^{2}\right]\left[\left(\tilde{P}_{\perp}+z q_{\perp}\right)^{2}+\epsilon_{M q}^{2}\right]}\right. \\
& \left.-z^{2}(1-z) M^{2}\left[\frac{1}{\tilde{P}_{\perp}^{2}+\epsilon_{M}^{2}}-\frac{1}{\left(\tilde{P}_{\perp}+z q_{\perp}\right)^{2}+\epsilon_{M}^{2}}\right]_{\text {PENNSTATE }}^{2}\right\}, \\
\text { Direct photon measurement. }
\end{array}
$$

Remarks:

- Correlation.

- In addition, test the BK evolution equation. 
Dilepton Pair + hadron correlation

Azimuthal angle correlation of $\gamma^{*}+\pi^{0}$ at forward rapidity 3.2:

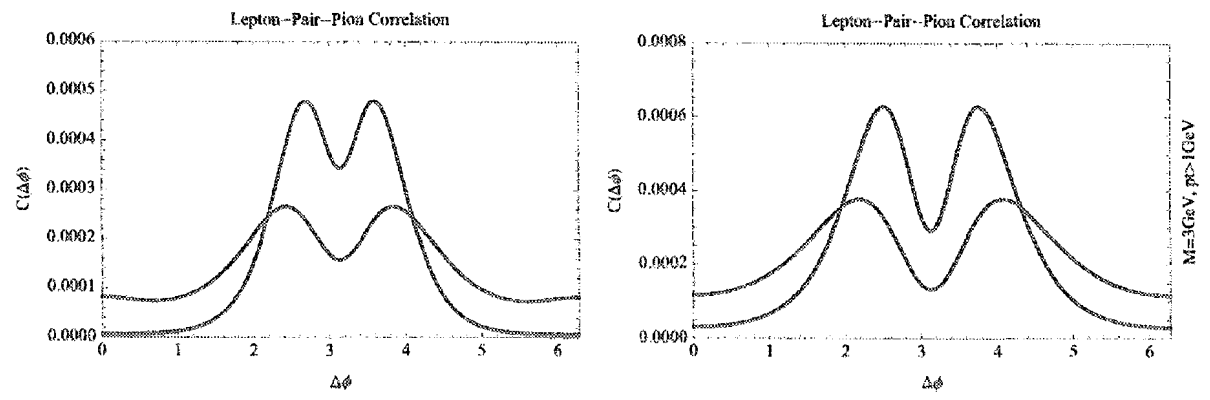

Remarks:

- $p_{1 \perp}>1.5 \mathrm{Gev}, p_{2 \perp}>1.5 \mathrm{Gev}$ and $M^{2}=1 \mathrm{GeV}^{2}$;

- $p_{1 \perp}>1 \mathrm{Gev}, p_{2 \perp}>1 \mathrm{Gev}$ and $M^{2}=9 \mathrm{GeV}^{2}$;

- Suppression of away side peak at central $d A u$ collisions.

- The unique double peak structure on the away side comes from the fact that $x G^{(2)} \propto q_{\perp}^{2}$ in the small $q_{\dot{j}}$ limit.

- To avoid the contamination of $\rho$ and $J / \Psi$, better choice of kinematical region. Low Mass $M^{2}$ vs high mass? 


\section{Questions?}

What is the theoretical uncertainties of DY AN predictions?

= How do they affect our goal of checking the sign change?

- What is the real impact of the measurement of sign change?

- Is this issue only relevant to spin physics? How should be convey to outside community?

- If we have sign change, what is the contribution we have made?

อ

- If we have not sign change, what does this mean? Is this really a big deal? 


\section{"Sign mismatch" between SIDIS and pp}

- Transition from low PT to high PT

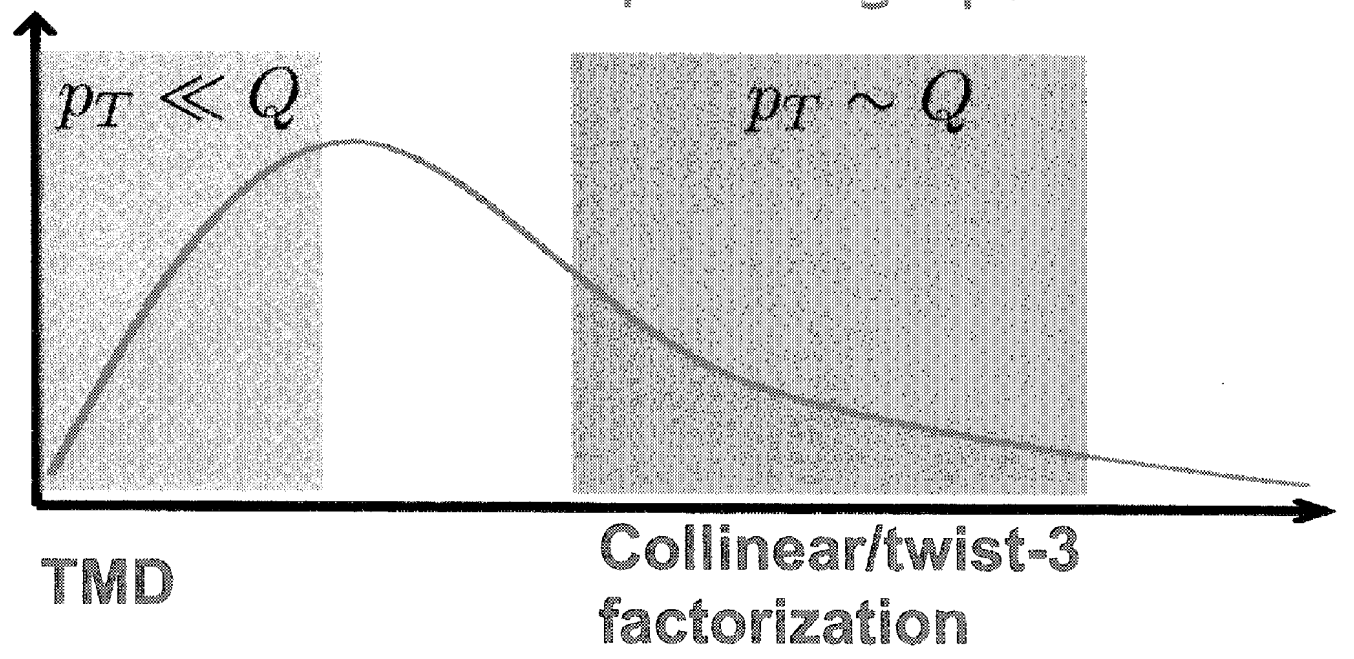

$\bar{\Xi}$

- Need to determine the sign and constrain $T_{F}(x, x)$
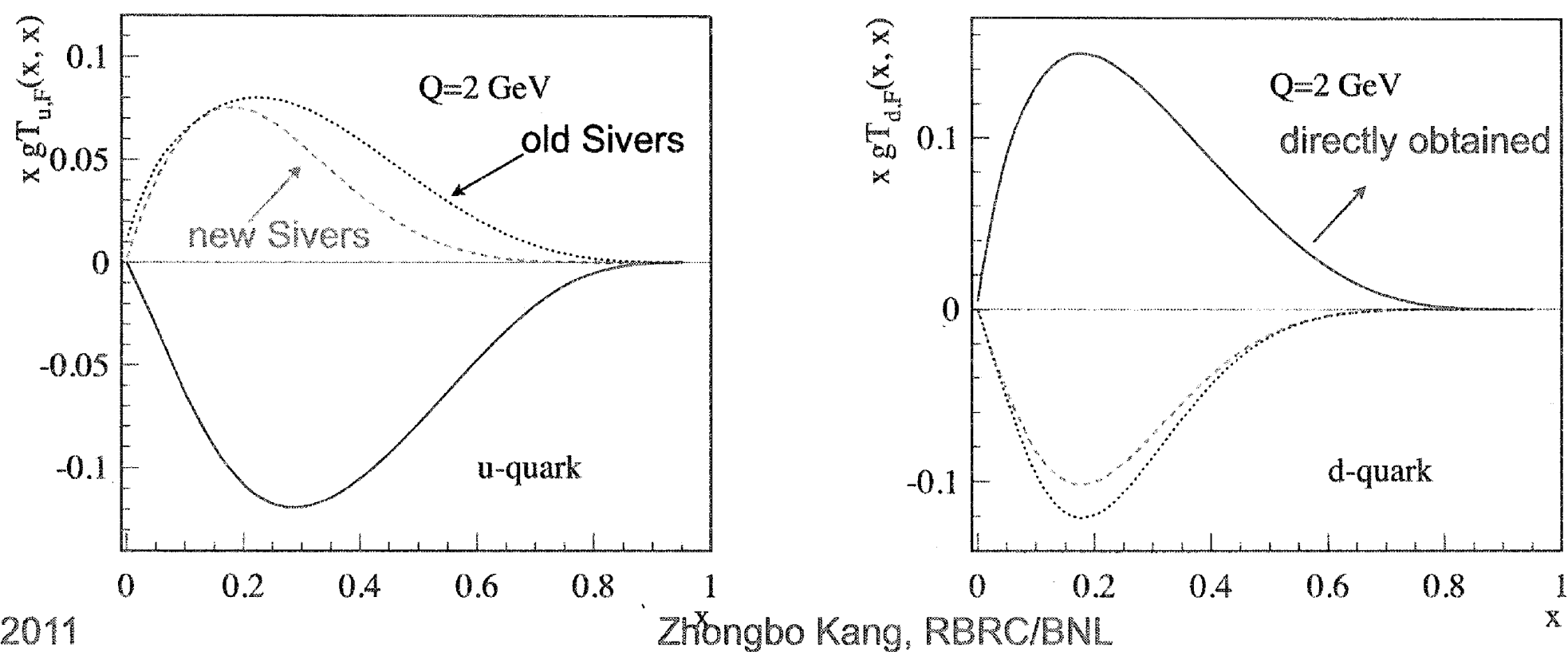

May 11,2011 Zhóngbo Kang, RBRC/BNL 


\section{Dijet asymmetry measurement}

- The theory prediction here is using $T_{F}(x, x)$ from the first kt-moment of Sivers function from SIDIS

$\underline{\Xi}$

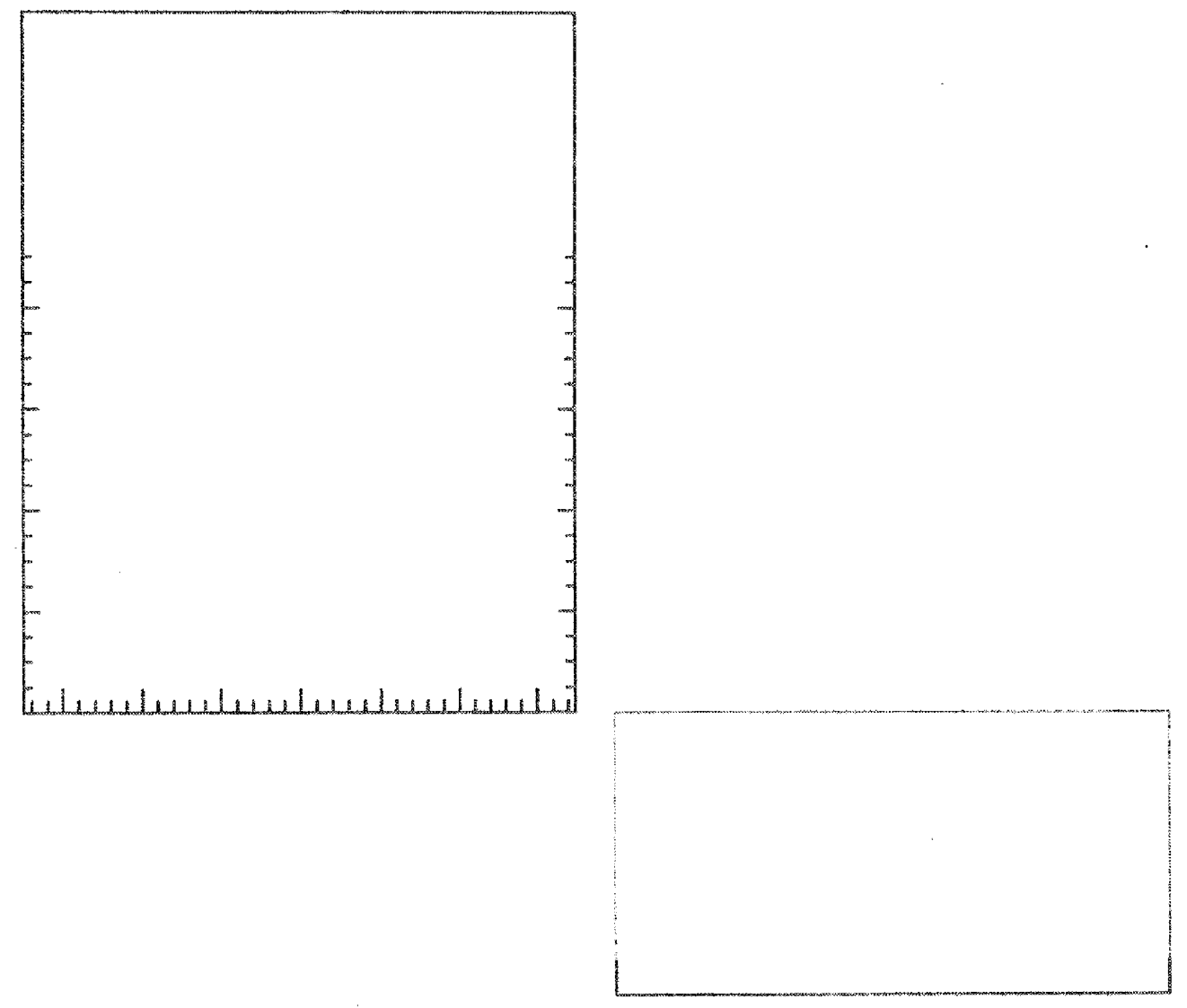

- Also the problem of factorization breaking 
n.

ก

$\omega$

in

5

$\frac{x}{\frac{1}{n}}$

0

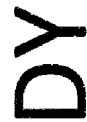

4

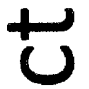

(0)

0

$E$

$\frac{0}{+\infty}$

U

$+3$

$\sum$

○ 
- What to blame if

- No sign change

- if we see a sign change but different magnitude/shape?

- What can we learn from Collider vs. fixed Target?

-What measurements are needed in the future?

- (or what analysis should be done on existing data? )

- What do we need to learn from current DY experiments (Compass, AnDY, E906) for the future 


\section{Still open: Jen-Chieh at 2010 DY workshop in Santa Fe}

- Is there a Boer-Mulders sign change?

- Boer-Mulders different in protons and pions?

- Flavor dependence of DY?

- $\mathrm{k}_{\mathrm{t}}$ dependence:

$-x$ dependence?

- flavor dependence?

- difference between nucleons and mesons?

- gluon/quark differences? 


\section{Generalized TMDs and Wigner Distributions}

(A. Metz, Department of Physics, Temple University, Philadelphia, PA 19122)

ABSTRACT: The first complete parameterization of Generalized TMDs (GTMDs) for a spin- $\frac{1}{2}$ target is presented. The Fourier transform of GTMDs has a strong similarity to Wigner distributions, which are the quantum mechanical analogues of classical phase space distributions.

Many nontrivial relations between GPDs and TMDs have been found in simple spectator models. Since GTMDs contain the GPDs and the TMDs in certain limits, one can use them in order to study the status of the nontrivial GPD-TMD relations. Such an analysis reveals that none of those relations can be promoted to a model-independent status. The talk also briefly addresses more recent developments on the GTMD field as well as some potential further applications of these objects.

In collaboration with: K. Goeke, S. Meißner, M. Schlegel

(hep-ph/0703176 ; arXiv:0805.3165 ; arXiv:0906.5323) 

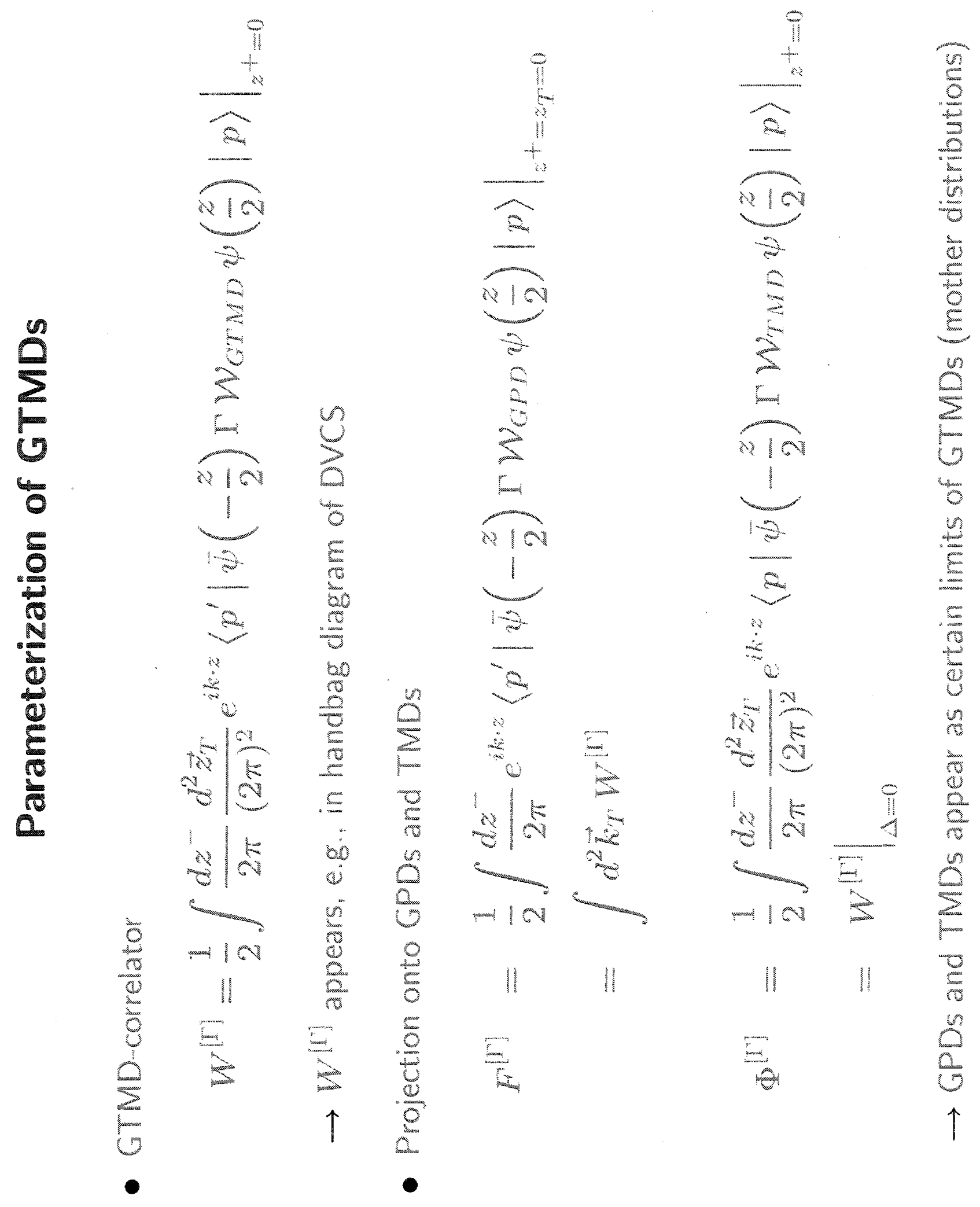

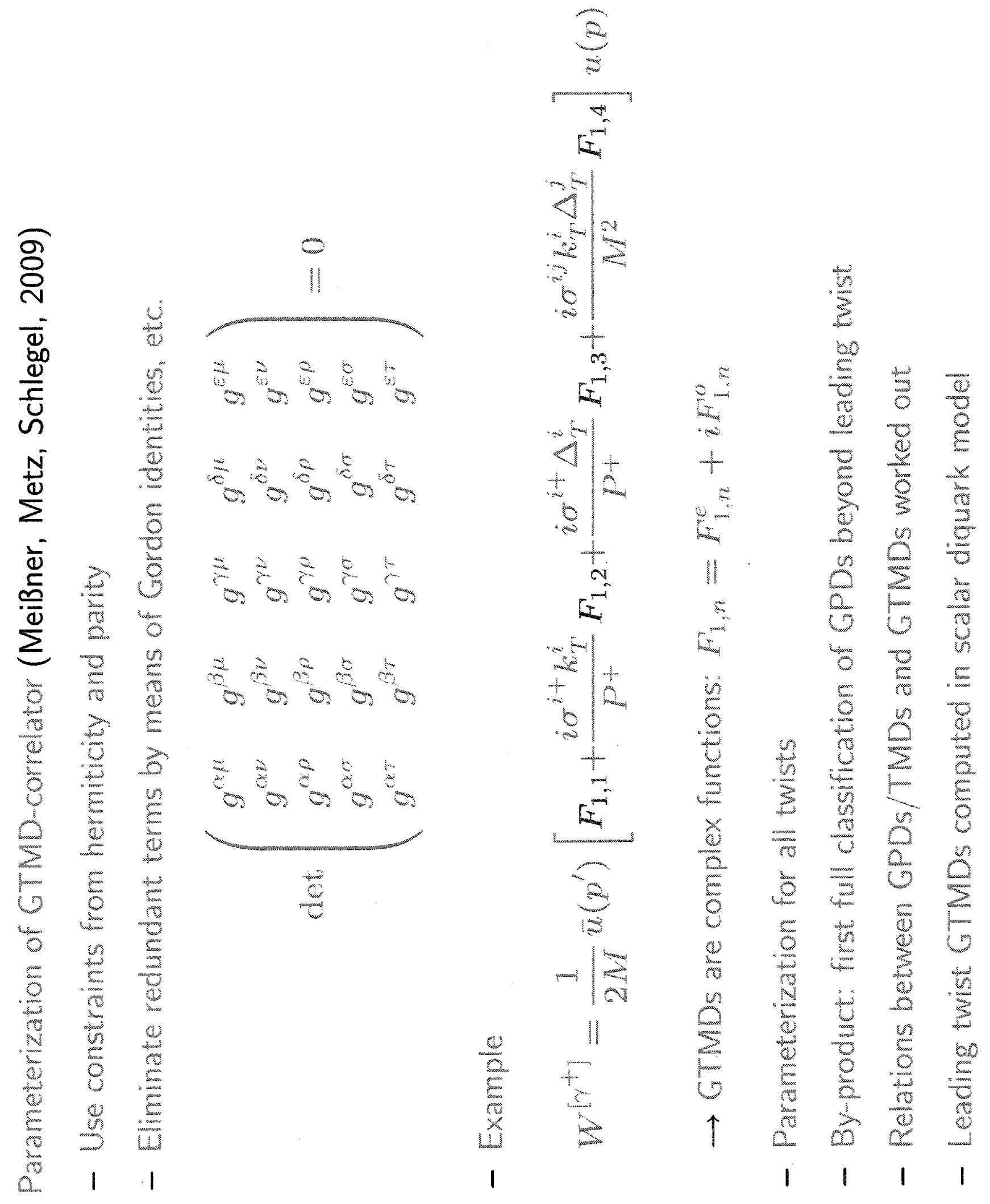


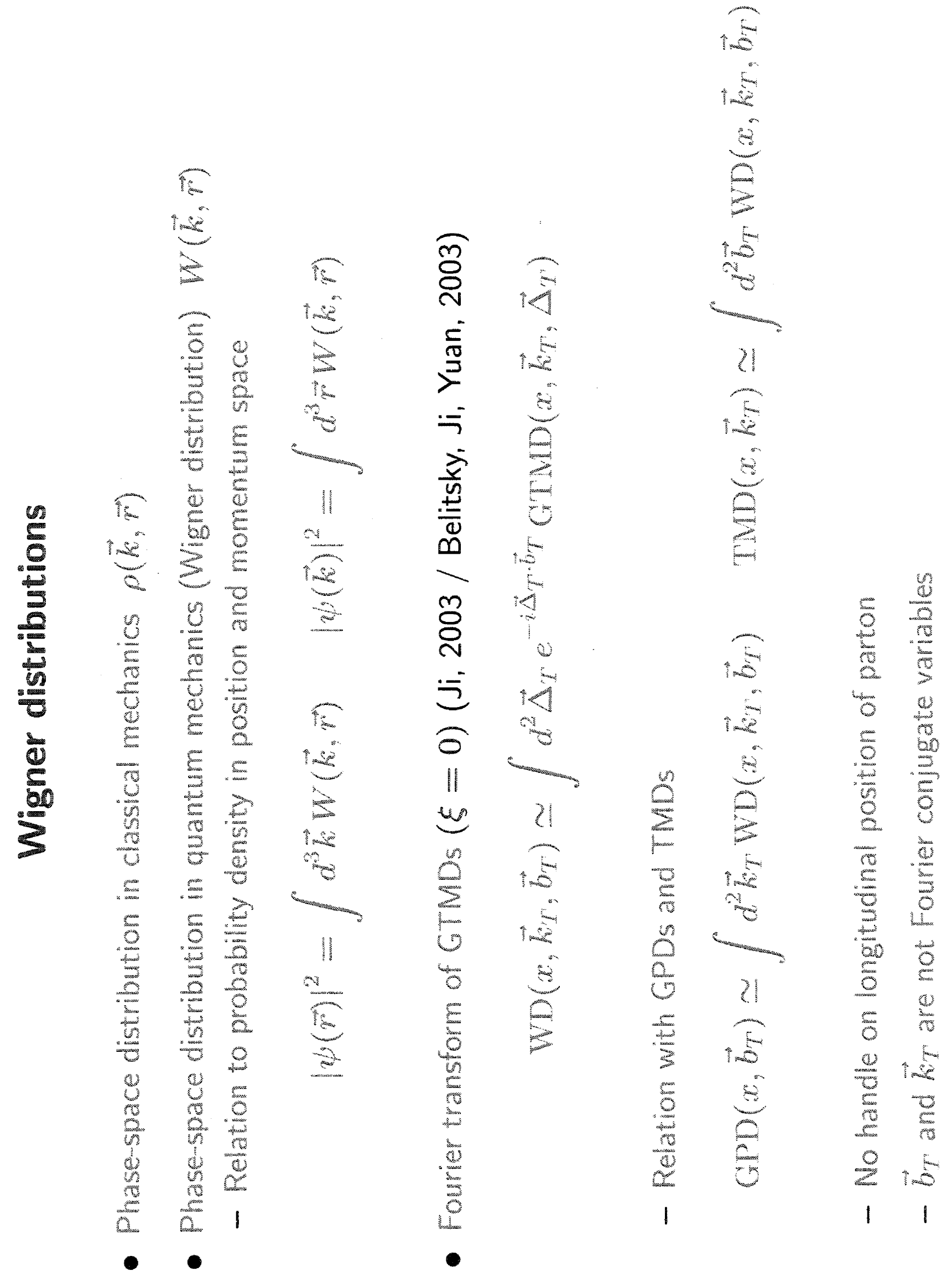




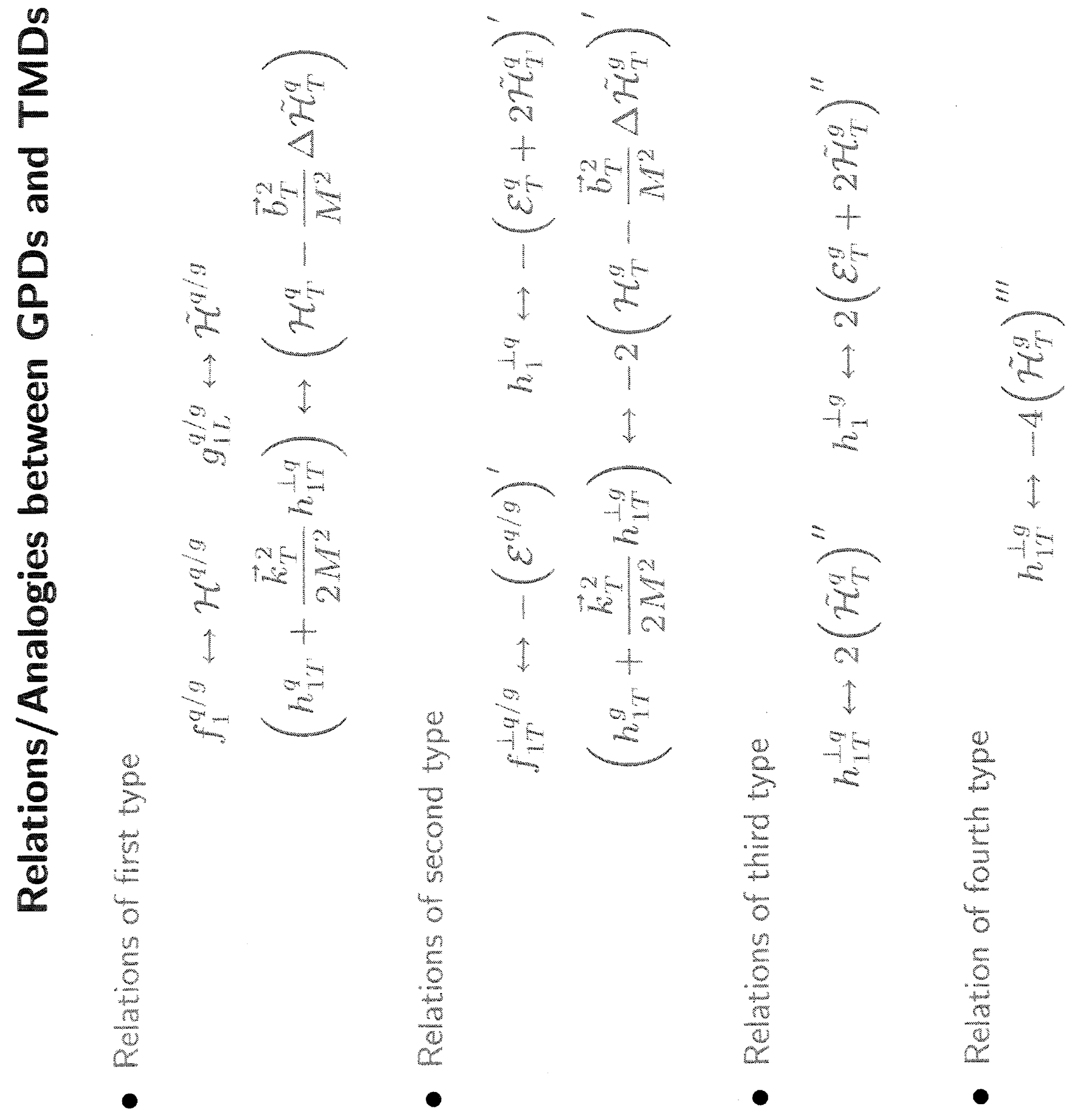




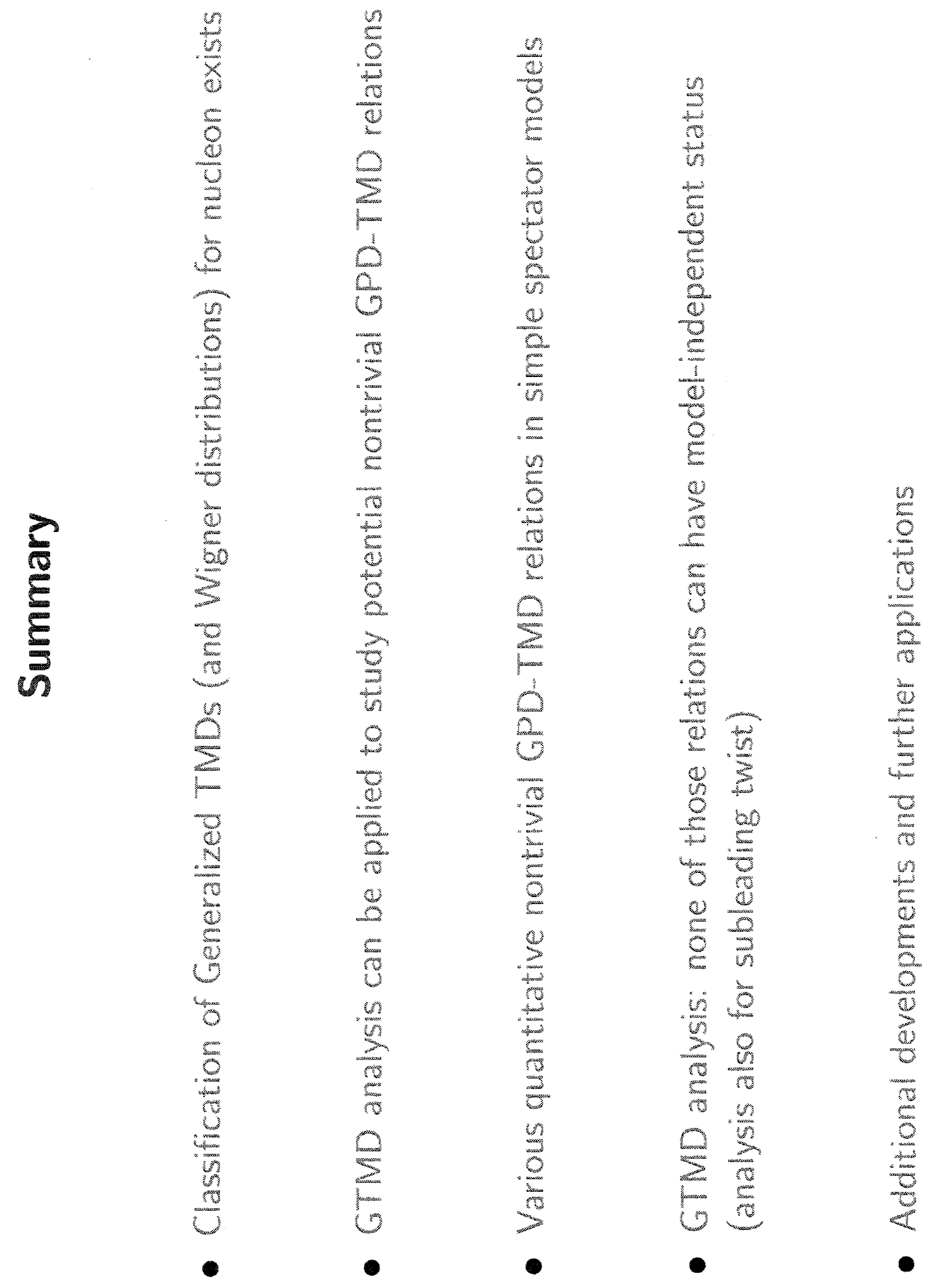




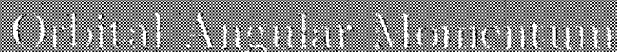

Matthias Burkardt

New Mexico State University

May 13, 2011 


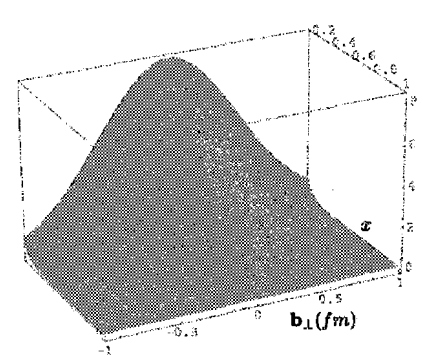

- $x=$ momentum fraction of the quark

- $\vec{b}=\perp$ distance of quark from $\perp$ center of momentum

- small $x$ : large 'meson cloud'

- larger $x$ : compact 'valence core'

- $x \rightarrow 1$ : active quark becomes center of momentum

... $\vec{b}_{\perp} \rightarrow 0$ (narrow distribution) 

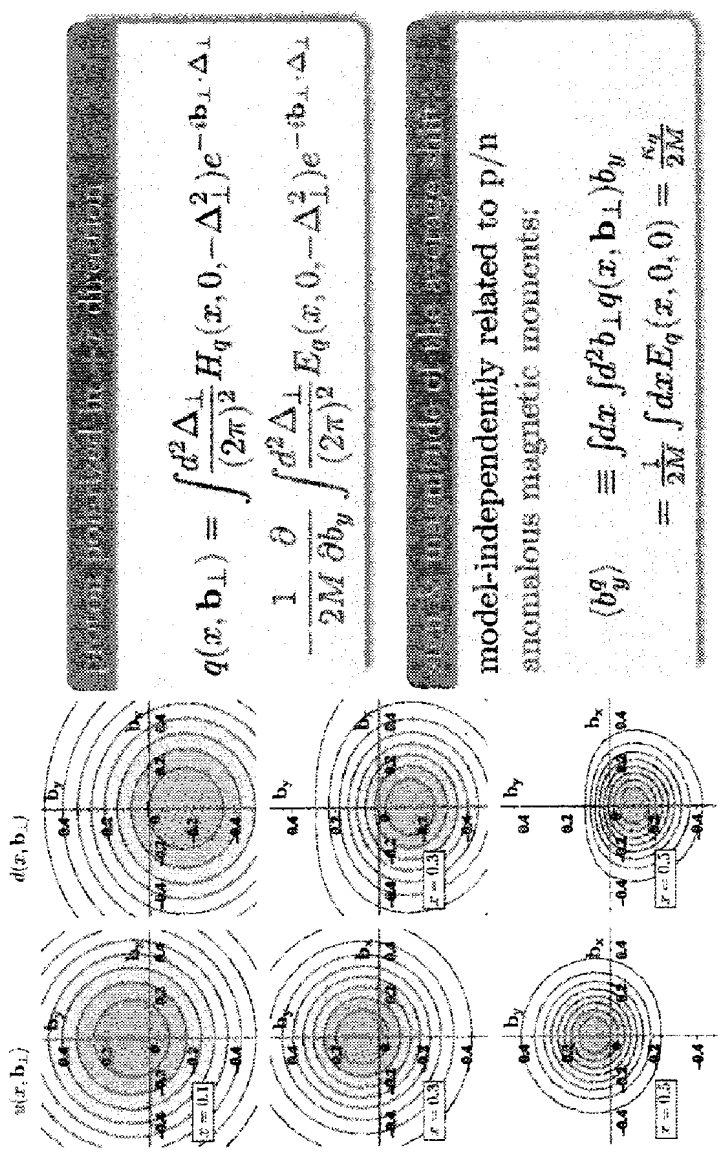


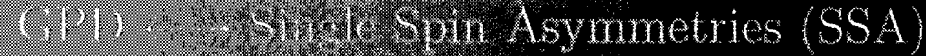

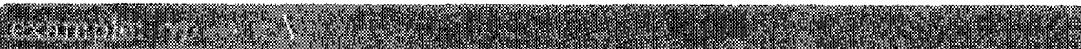

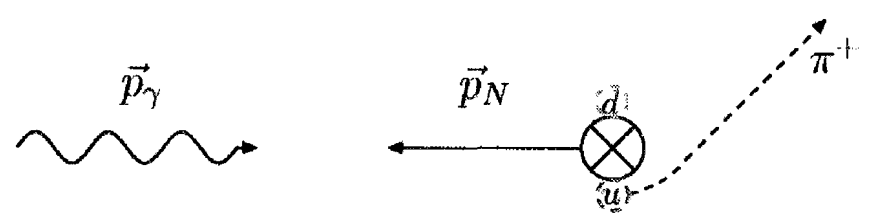

- $u, d$ distributions in $\perp$ polarized proton have left-right asymrnetry in $\perp$ position space (T-even!); sign 'determined' by $\kappa_{u} \& \kappa_{d}$

- attractive FSI deflects active quark towards the CoM

$\because$ FSI translates position space distortion (before the quark is knocked out) in $+\hat{y}$-direction into momentum asymmetry that favors $-\hat{y}$ direction $\rightarrow$ 'chromodynamic lensing'

$\Rightarrow$

$\kappa_{p}, \kappa_{\text {俩 }} \longleftrightarrow$ sign of SSAMIIMI $(\mathrm{MB}, 2004)$

- confirmed by HERMEs $p$ data; consistent with vanishing isoscalar Sivers (COMPASs) 


\section{Imon him Jughem Carried by Quarks}

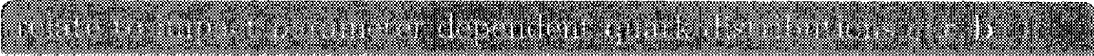

- Thus $f_{q}^{x}=m_{N} \int d x x^{y} q\left(x, \mathbf{r}_{\perp}\right)$ with $b^{y}=r^{y}-\frac{1}{2 m_{N}}$, where $q\left(x, r_{1}\right)$ is distribution relative to CoM of whole nucleon

- recall: $q\left(x, \mathbf{b}_{1}\right)$ for nucleon polarized in $+\hat{x}$ direction

$$
\begin{aligned}
& q\left(x, \mathbf{b}_{\perp}\right)=\int \frac{d^{2} \Delta_{ \pm}}{(2 \pi)^{2}} H_{q}\left(x, 0,-\Delta_{\perp}^{2}\right) e^{-\mathbf{i} \mathbf{b}_{\perp} \Delta_{\perp}} \\
& -\frac{1}{2 M_{N}} \frac{\partial}{\partial h_{y}} \int \frac{d^{2} \Delta}{(2 \pi)^{2}} E_{q}\left(x, 0,-\Delta_{1}^{2}\right) e^{-h_{H}-\Delta_{L}} \\
& \Rightarrow x_{a}^{x}=M_{N} \int d x r^{y} q\left(x, \mathbf{r}_{1}\right)=\int d x x\left(m_{N} b^{y}+\frac{1}{2}\right) q\left(x, \mathbf{r}_{1}\right) \\
& =\frac{1}{2} \int d x x[H(x, 0,0)+E(x, 0,0)]
\end{aligned}
$$

- X.Ji (1996): rotational invariance $\Rightarrow$ apply to all components of $\vec{J}$ - partonic interpretation exists only for $\perp$ components! 


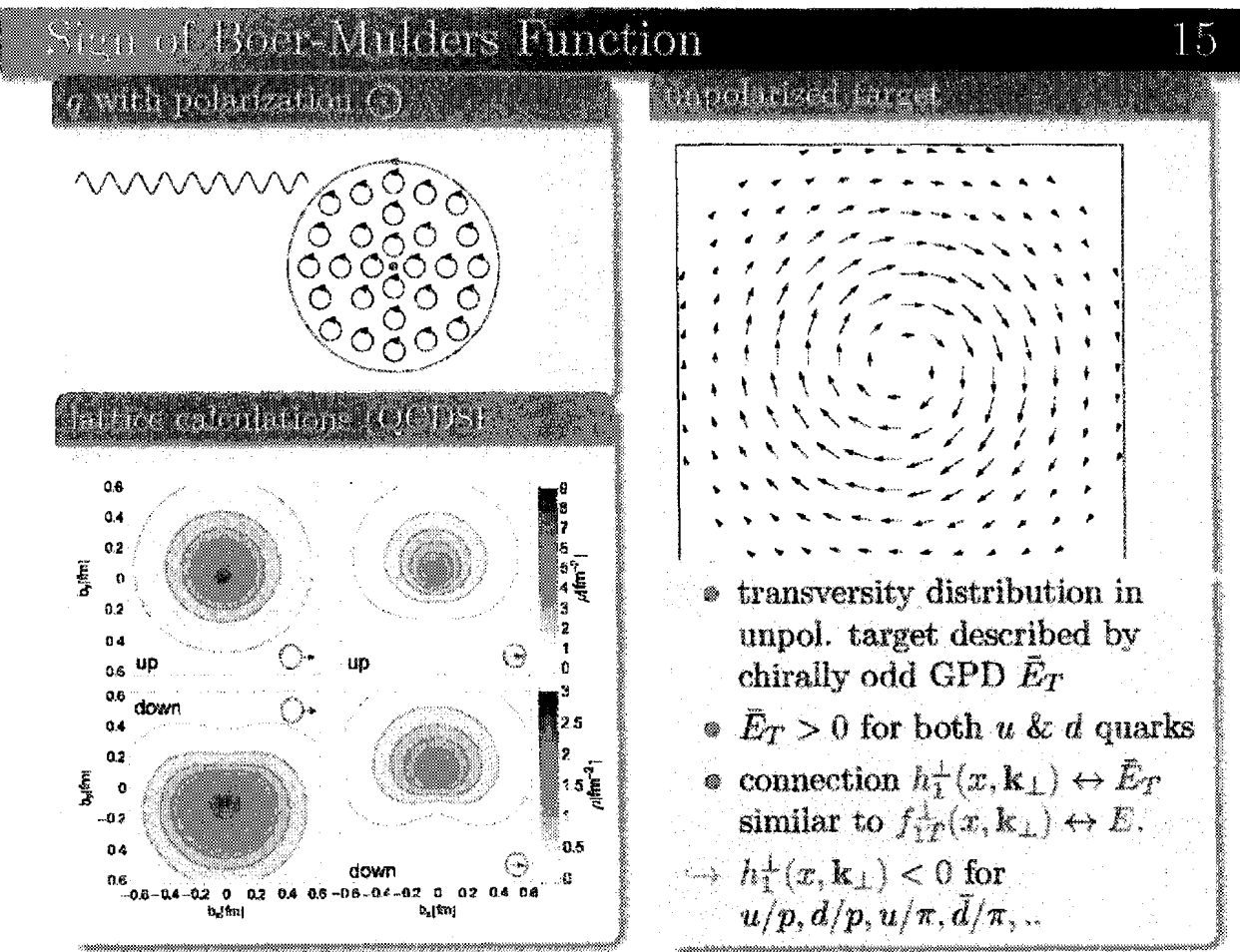




\title{
Probing multi-gluon correlations in pp collisions
}

\author{
Yuji Koike
}

Department of Physics, Niigata University, Ikarashi, Niigata 950-2181, Japan

\section{Abstract:}

- We derived the contribution of the 3-gluon correlation functions to the polarized cross section for $p^{\uparrow} p \rightarrow D X, p^{\uparrow} p \rightarrow \gamma X$ and $p^{\uparrow} p \rightarrow \ell^{+} \ell^{-} X$.

- There are two independent twist-3 three-gluon correlation functions in the polarized nucleon due to the different color contractions; $O\left(x_{1}, x_{2}\right)$ and $N\left(x_{1}, x_{2}\right)$.

- SSA occurs as a pole contribution which is written in terms of four independent functions $O(x, x), N(x, x), O(x, 0)$ and $N(x, 0)$.

- Numerical calculation for $p^{\uparrow} p \rightarrow D X$ and $p^{\uparrow} p \rightarrow \gamma X$.

- Rising behavior of $A_{N}$ for $p^{\uparrow} p \rightarrow D X$ at $x_{F}>0$ as in the case of the SGP contribution from the quark-gluon correlation function for $p^{\uparrow} p \rightarrow \pi X$.

- For $p^{\uparrow} p \rightarrow \gamma X, A_{N} \simeq 0$ at $x_{F}>0$ regardless of the magnitude of the 3-gluon correlation functions.

- $A_{N}$ at $x_{F}<0$ is sensitive to small- $x$ behavior of 3-gluon correlation function for the two processes.

* Two processes are useful to get costraint on magnitude and shape of 3-gluon correlation functions. 
* Twist-3 "three-gluon" correlation functions

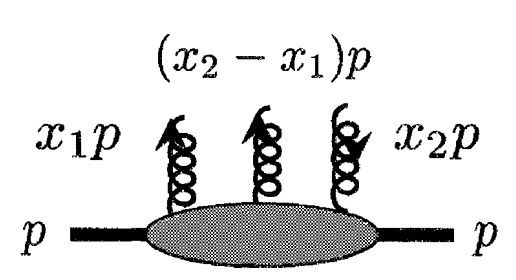

cf. Beppu-Koike-Tanaka-Yoshida (PRD 82('10)054005)

See also, Belitsky-Ji-Lu-Osborne, PRD63,094012(2001)

Braun-Manashov-Pirnay, PRD80,114002(2009).

- Hermiticity, PT-invariance, Permutation symmetry

$$
\begin{aligned}
O^{\alpha \beta \gamma}\left(x_{1}, x_{2}\right) & =-g i^{3} \int \frac{d \lambda}{2 \pi} \int \frac{d \mu}{2 \pi} e^{i \lambda x_{1}} e^{i \mu\left(x_{2}-x_{1}\right)}\left\langle p S\left|d^{b c a} F_{b}^{\beta n}(0) F_{c}^{\gamma n}(\mu n) F_{a}^{\alpha n}(\lambda n)\right| p S\right\rangle \\
= & 2 i M_{N}\left[O\left(x_{1}, x_{2}\right) g^{\alpha \beta} \epsilon^{\gamma p n S}+O\left(x_{2}, x_{2}-x_{1}\right) g^{\beta \gamma} \epsilon^{\alpha p n S}+O\left(x_{1}, x_{1}-x_{2}\right) g^{\gamma(x)} \epsilon^{\beta p n S}\right]
\end{aligned}
$$

E

$$
\begin{aligned}
N^{\alpha \beta \gamma}\left(x_{1}, x_{2}\right) & =-g i^{3} \int \frac{d \lambda}{2 \pi} \int \frac{d \mu}{2 \pi} e^{i \lambda x_{1}} e^{i \mu\left(x_{2}-x_{1}\right)}\left\langle p S\left|i f^{b c \alpha} F_{b}^{\beta n}(0) F_{c}^{\gamma n}(\mu n) F_{a}^{\alpha n}(\lambda n)\right| p S\right\rangle \\
= & 2 i M_{N}\left[N\left(x_{1}, x_{2}\right) g^{\alpha \beta} \epsilon^{\gamma p n S}-N\left(x_{2}, x_{2}-x_{1}\right) g^{\beta \gamma} \epsilon^{\alpha p n S}-N\left(x_{1}, x_{1}-x_{2}\right) g^{\gamma \alpha} \epsilon^{\beta p n S}\right] . \\
F_{a}^{\alpha n} & \equiv F_{a}^{\alpha \mu} n_{\mu} \quad n \text { : lightlike vector satisfying } p \cdot n=1 . \\
\epsilon^{\gamma p n S} & \equiv \epsilon^{\gamma \mu \nu \lambda} p_{\mu} n_{\nu} S_{\lambda} \text { etc. }
\end{aligned}
$$

- Only two independent scalar functions due to the different color structures:

$$
\begin{aligned}
& O\left(x_{1}, x_{2}\right)=O\left(x_{2}, x_{1}\right), \quad O\left(x_{1}, x_{2}\right)=O\left(-x_{1},-x_{2}\right), \\
& N\left(x_{1}, x_{2}\right)=N\left(x_{2}, x_{1}\right), \quad N\left(x_{1}, x_{2}\right)=-N\left(-x_{1},-x_{2}\right) .
\end{aligned}
$$



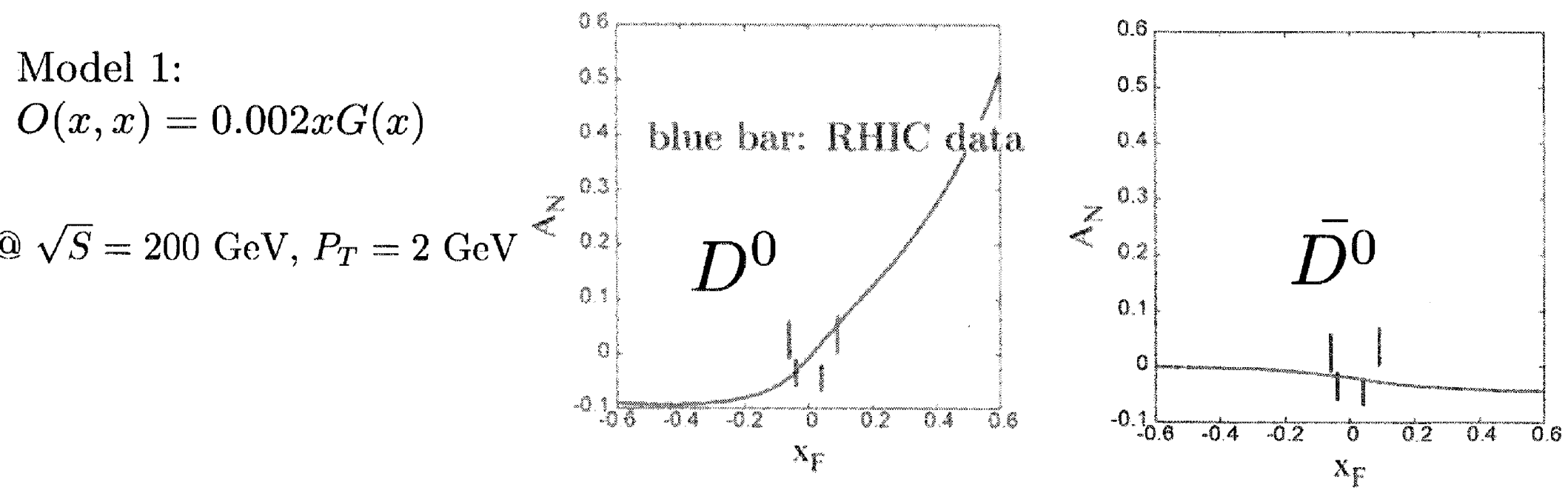

- Change of relative signs between $\{O(x, x), O(x, 0)\}$ and $\{N(x, x), N(x, 0)\}$ gives opposite prediction for $D$ and $\bar{D}$ mesons.

\section{Model 2:}

$O(x, x)=\frac{1}{4} \times 0.002 \sqrt{x} G(x)$
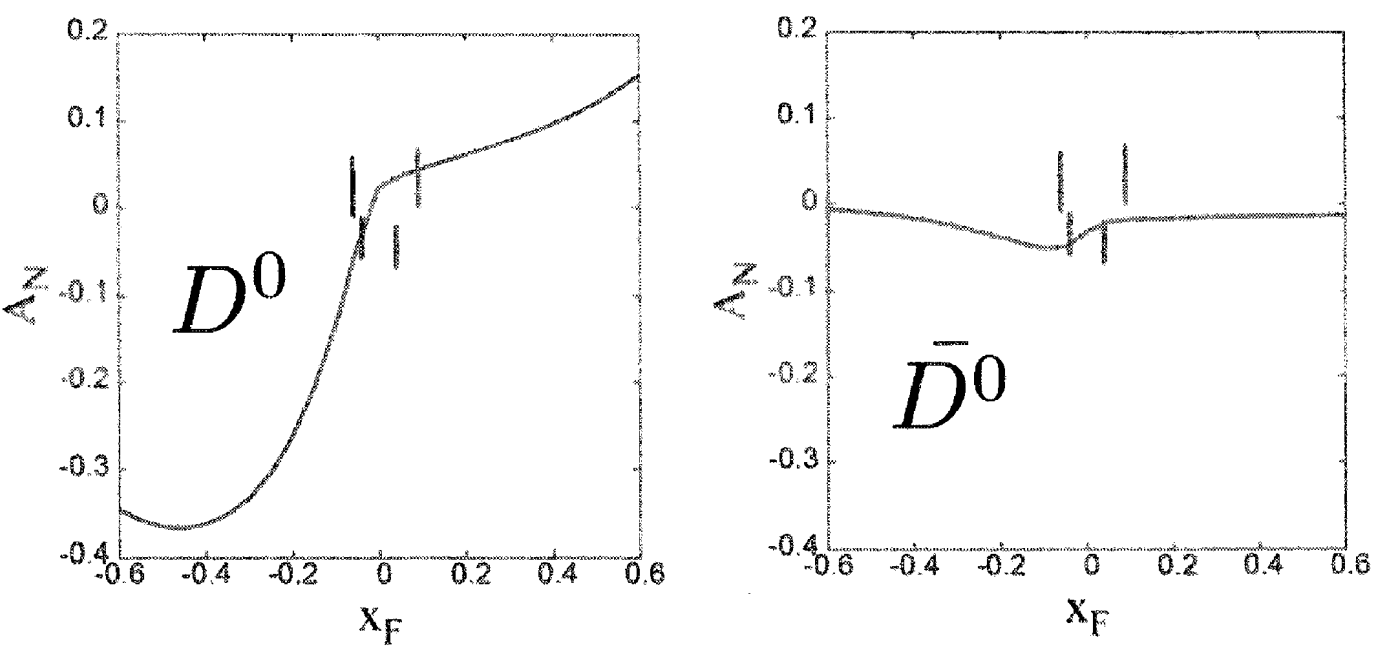

- $A_{N}$ at $x_{F}<0$ strongly depends on the small- $x$ behavior of 3 -gluon correlation function. 
* Threc-gluon contribution to the direct photon production: $p^{\uparrow}(p)+p(p) \rightarrow \gamma(q)+$ $X$.

$$
\begin{aligned}
& E_{\gamma} \frac{d \sigma}{d^{3} q}= \frac{4 \alpha_{e m} \alpha_{s} M_{N} \pi}{S} \sum_{a} \int \frac{d x^{\prime}}{x^{\prime}} f_{a}\left(x^{\prime}\right) \int \frac{d x}{x} \delta(\hat{s}+\hat{t}+\hat{u}) \epsilon^{q p n S_{\perp}} \frac{1}{\hat{u}} \\
& \times\left[\delta_{a}\left(\frac{d}{d x} O(x, x)-\frac{2 O(x, x)}{x}+\frac{d}{d x} O(x, 0)-\frac{2 O(x, 0)}{x}\right)\right. \\
&\left.\quad-\frac{d}{d x} N(x, x)+\frac{2 N(x, x)}{x}+\frac{d}{d x} N(x, 0)-\frac{2 N(x, 0)}{x}\right]\left(\frac{1}{N}\left(\frac{\hat{s}}{\hat{u}}+\frac{\hat{u}}{\hat{s}}\right)\right)
\end{aligned}
$$

$\delta_{a}=1$ for $a=$ quark, $\delta_{a}=-1$ for $a=$ anti-quark.

$$
\hat{s}=\left(x p+x^{\prime} p^{\prime}\right)^{2}, \hat{t}=(x p-q)^{2}, \hat{u}=\left(x^{\prime} p^{\prime}-q\right)^{2}
$$

The same as twist-2 cross section (also from master formula!)

- This differs form the previous study (X. Ji, Phys.lett.B289 ('92)137).

- Contribute in the combination of $O(x, x)+O(x, 0)$ and $N(x, x)-N(x, 0)$ as in $m_{c} \rightarrow 0$ for $p^{\uparrow} p \rightarrow D X$.

$. O(x, x)+O(x, 0)=-(N(x, x)-N(x, 0)) \rightarrow$ quarks in the unpolarized nucleon are active. $\rightarrow$ Large $A_{N}^{\gamma}$.

- $O(x, x)+O(x, 0)=N(x, x)-N(x, 0) \rightarrow$ quarks in the unpolarized nucleon are NOT active. $\rightarrow$ Small $A_{N}^{\gamma}$. 
Change sign for $N$ as $N(x, x) \rightarrow-N(x, x)$ and $N(x, 0) \rightarrow-N(x, 0)$.

$\rightarrow$ Quark contribution from the unpolarized nucleon is active, while anti-quark contribution is cancelled.
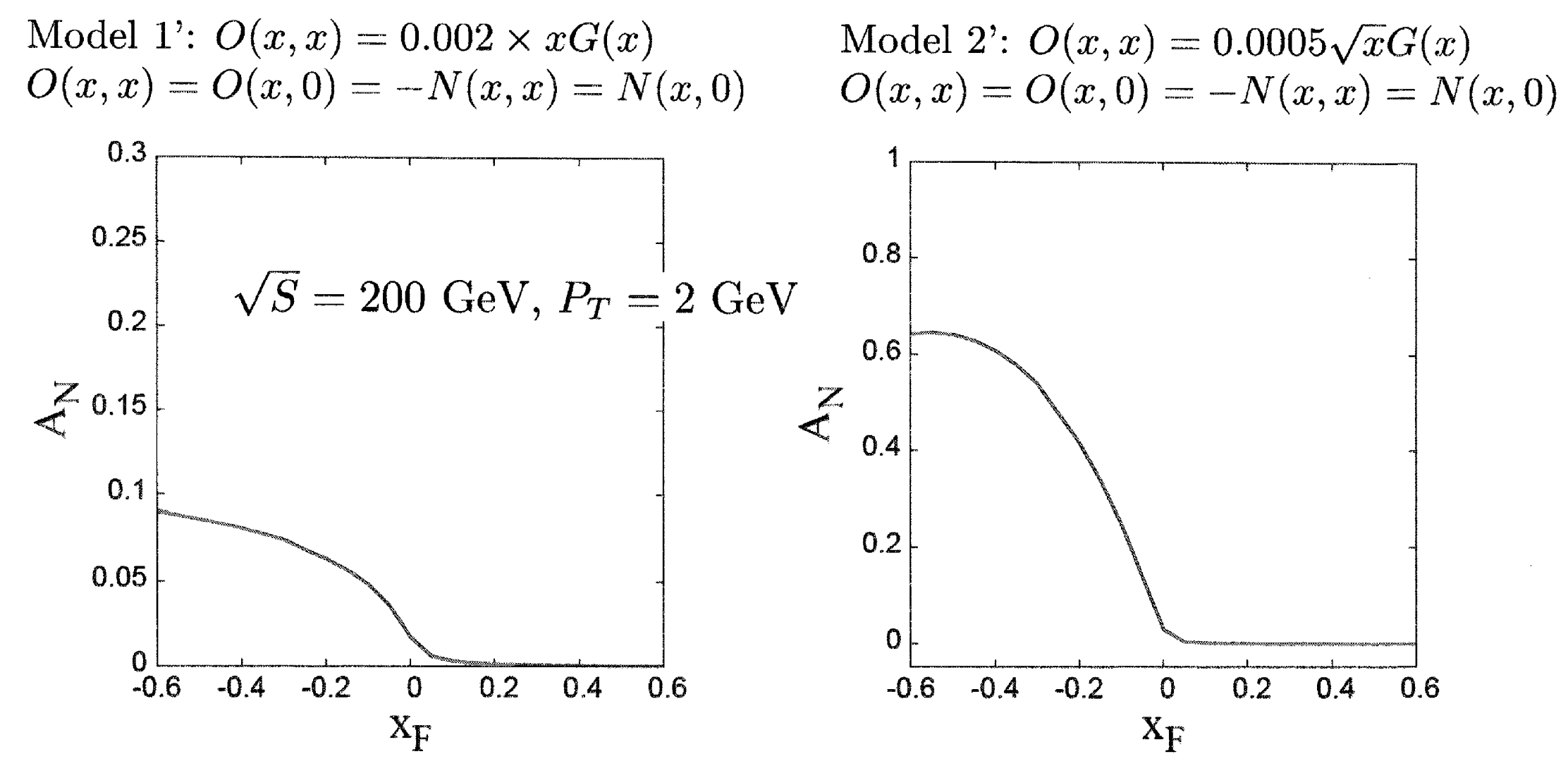

- $A_{N} \sim 0$ at $x_{F}>0$ regardless of magnitude of the 3 -gluon correlation functions.

- Behavior at $x_{F}<0$ is sensitive to small $x$ behavior similarly to $p p \rightarrow D X$. 
* Three-gluon contribution to $p^{\uparrow} p \rightarrow \gamma^{*} X . \quad$ (YK, S.Yoshida, in preparation)

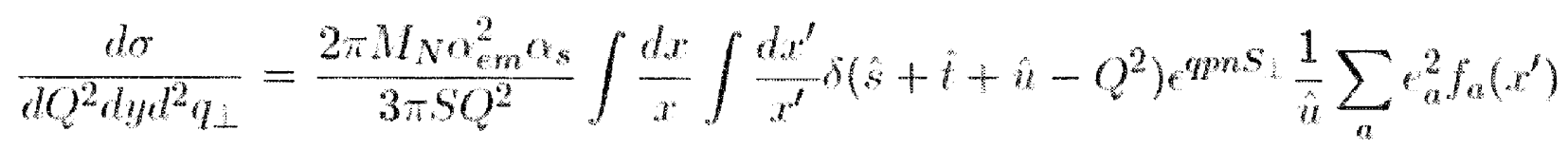

$$
\begin{aligned}
& \times\left[\delta_{a}\left(\frac{d}{d x} O(x, x)-\frac{2 O(x, x)}{x}\right) \hat{\sigma}_{1}+\left(\frac{d}{d x} O(x, 0)-\frac{2 O(x, 0)}{x}\right) \hat{\sigma}_{2}+\frac{O(x . x)}{x} \hat{\sigma}_{3}+\frac{O(x, 0)}{x} \hat{\sigma}_{4}\right. \\
& \left.-\left(\frac{d}{d x} N(x, x)-\frac{2 N(x, x)}{x}\right) \hat{\sigma}_{1}+\left(\frac{d}{d x} N(x, 0)-\frac{2 N(x, 0)}{x}\right) \hat{\sigma}_{2}-\frac{N(x, x)}{x} \hat{\sigma}_{3}+\frac{N(x, 0)}{x} \hat{\sigma}_{4}\right] \\
& \hat{\sigma}_{1}=\frac{2}{N}\left(\frac{\hat{u}}{\hat{s}}+\frac{\hat{s}}{\hat{u}}+\frac{2 Q^{2} \hat{f}}{\hat{s} \hat{u}}\right) \quad \hat{\sigma}_{3}=-\frac{1}{N} \frac{4 Q^{2}\left(Q^{2}+\hat{t}\right)}{\hat{s i u}} \\
& \hat{\sigma}_{2}=\frac{2}{N}\left(\frac{\hat{u}}{\hat{s}}+\frac{\hat{s}}{\hat{u}}+\frac{4 Q^{2} \hat{t}}{\hat{s} \hat{u}}\right) \quad \hat{\sigma}_{4}=-\frac{1}{N} \frac{4 Q^{2}\left(3 Q^{2}+t\right)}{\hat{s} \hat{u}}
\end{aligned}
$$

- At $Q^{2} \neq 0$, hard cross sections for $\{O(x, x), N(x, x)\}$ differ from those for $\{O(x, 0), N(x, 0)\}$ as in $e p^{\uparrow} \rightarrow e D X$.

- As $Q^{2} \rightarrow 0$, this agrees with the result for the direct-photon production.

- Sum of the above result and that from the quark-gluon correlation functions gives the complete twist-3 cross section for Drell-Yan and direct-photon processes.

For q-g correlations, see SGP: Ji-Qiu-Vogelsang-Yuan, PRD73('06), YK-Tanaka,PLB646('07) Hard pole+SFP: Kanazawa-YK,arXiv:1105.1036 [hep-ph] 


\section{May 2011 \\ Gauge Links \& Process dependence in Hadronic Reactions Leonard Gamberg Penn State University \\ Phys.Lett. B696 2011 w/ Zhongbo Kang BNL}

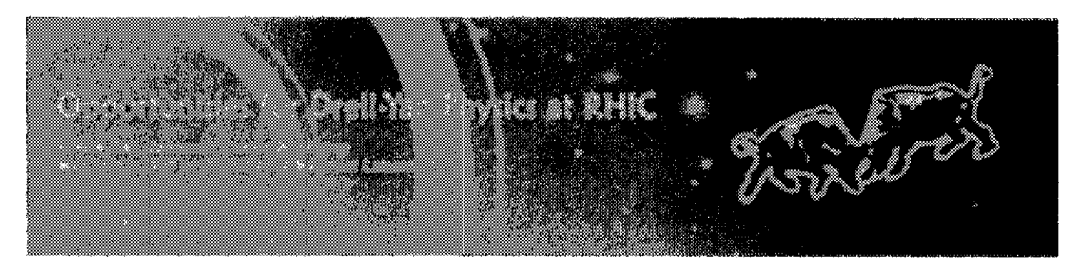

We study the single transverse spin asymmetries in the single inclusive particle production within the framework of the generalized parton model (GPM). By carefully analyzing the initial- and final-state interactions, we include the process-dependence of the Sivers functions into the GPM formalism. The modified GPM formalism has a close connection with the collinear twist-3 approach. Within the new formalism, we make predictions for inclusive $\pi^{0}$ and direct photon productions at RHIC energies. Also we consider the Sivers asymmetry from the cross section for $p^{\uparrow} p \longrightarrow h_{1}$ jet $X$ ( $w /$ D'lesio, Murgia \& Pisano). We find the predictions are opposite to those in the conventional GPM approach. 


\section{Spin Dependent Cross Section in GPM $p p \rightarrow \pi X$}

$$
f_{q / A^{\uparrow}}\left(x, \vec{k}_{T}\right)=f_{q / A}\left(x, k_{T}^{2}\right)+\frac{1}{2} \Delta^{N} f_{q / A^{\uparrow}}\left(x, k_{T}^{2}\right) \vec{S} \cdot\left(\hat{P} \times \vec{k}_{T}\right)
$$

$A_{N}$ is defined by the ratio $\quad A_{N} \equiv E_{h} \frac{d \Delta \sigma}{d^{3} P_{h}} / E_{h} \frac{d \sigma}{d^{3} P_{h}}$.

$$
\begin{aligned}
& E_{h} \frac{d \Delta \sigma}{d^{3} P_{h}}=\frac{\alpha_{s}^{2}}{S} \sum_{a, b, c} \int \frac{d x_{a}}{x_{a}} d^{2} k_{a}\left(\Delta^{N} f_{a / A}\left(x_{a}\right) h_{a T}\right) \frac{1}{2} S_{A} \cdot\left(\hat{P}_{A} \times \hat{k}_{a T}\right) \int \frac{d x_{b}}{x_{b}} d^{2} k_{b T} f_{b / B}\left(x_{b}, k_{b T}\right) \\
& \text { ‡ } \times \int \frac{d z_{c}}{z_{r}^{2}} D_{h / c}\left(z_{c}\right) H_{a b \rightarrow c}^{U}(\hat{s}, \hat{t}, \hat{u}) \delta(\hat{s}+\hat{t}+\hat{u}),
\end{aligned}
$$

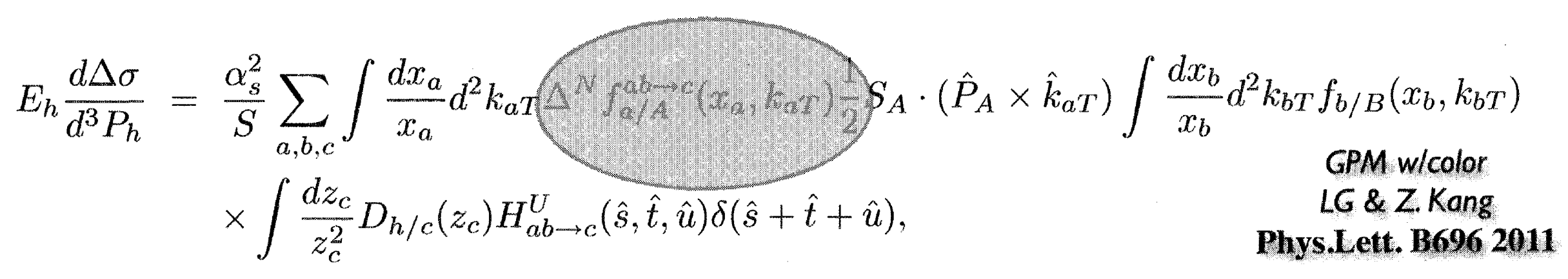

process-dependent Sivers function denoted as $\Delta^{N} f_{a / A}^{a b \rightarrow c}\left(x_{a}, k_{a T}\right)$ 


\section{One gluon exchange approx for ISI and FSI $q q^{\prime} \rightarrow q q^{\prime}$}

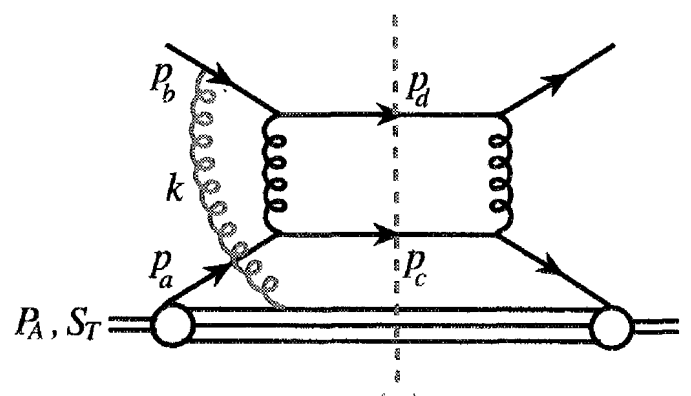

(a)

$\left[\frac{-g}{-k^{+}-i \epsilon} T^{a}\right]$

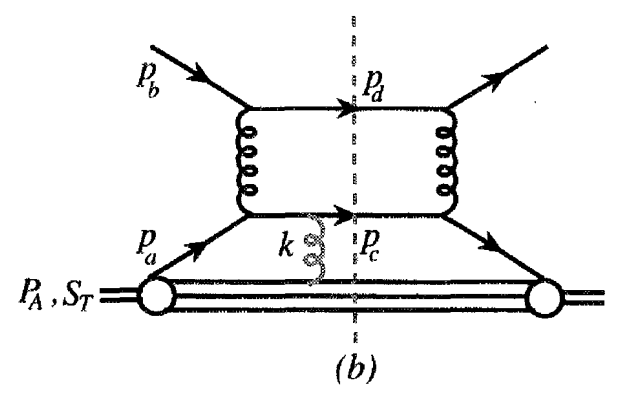

(b)

$\left[\frac{g}{-k^{+}+i \epsilon} T^{a}\right]$

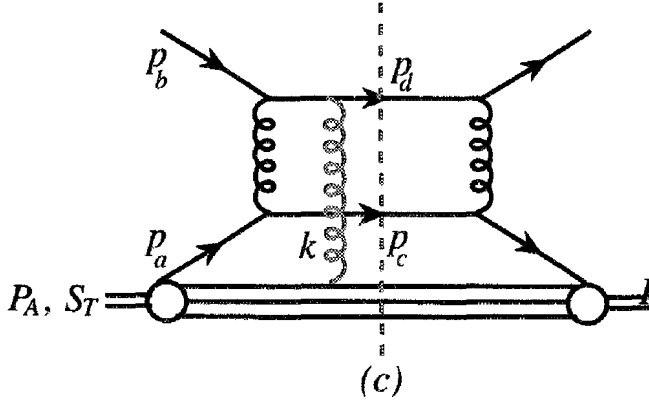

(c)

interaction w/unobserved particle "d" vanishes after summing over both cuts

E
$C_{I}=-\frac{1}{2 N_{c}^{2}}$,
$C_{F_{c}}=-\frac{1}{4 N_{c}^{2}}$,

calculate color factors

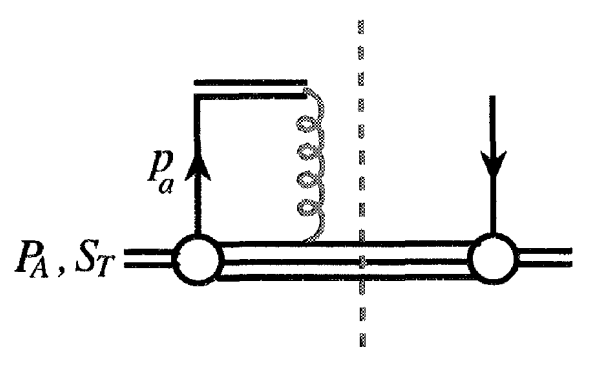

(a)

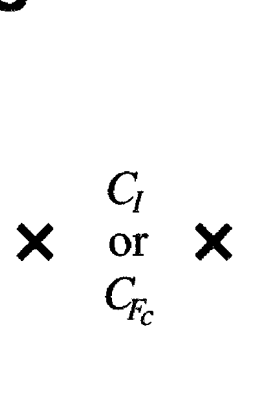

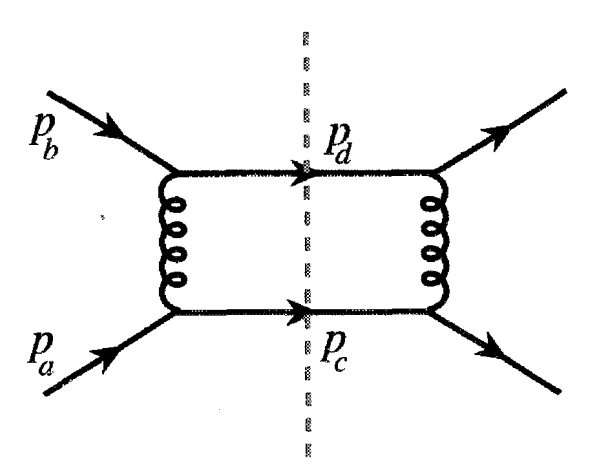

(b)
$C_{u}=\frac{N_{c}^{2}-1}{4 N_{c}^{2}}$
Note unpolarized color factor 


\section{Based on old parameterization Based on new parameterization}

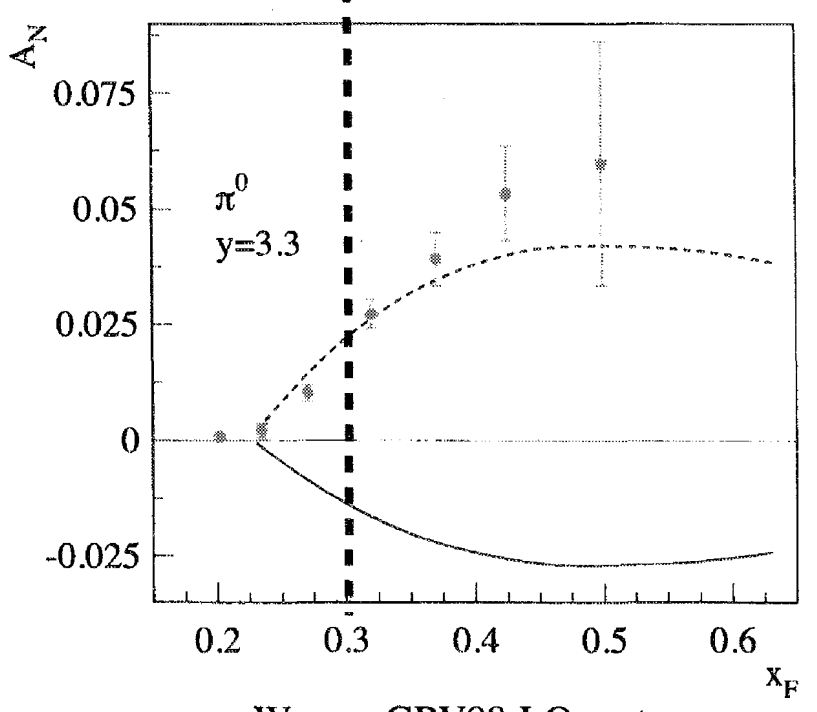

We use GRV98 LO parton
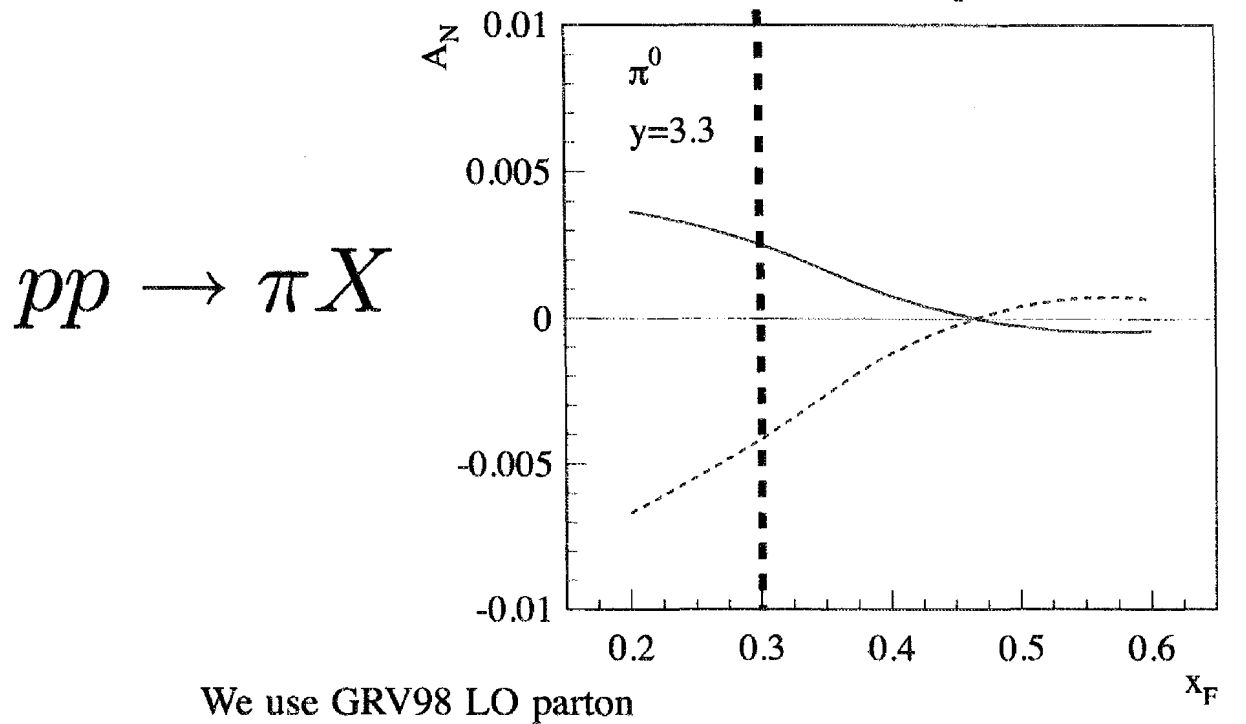

We use GRV98 LO parton

$\bar{\infty}$

the old Sivers function from [4], and Kretzer fragmentation function [5]

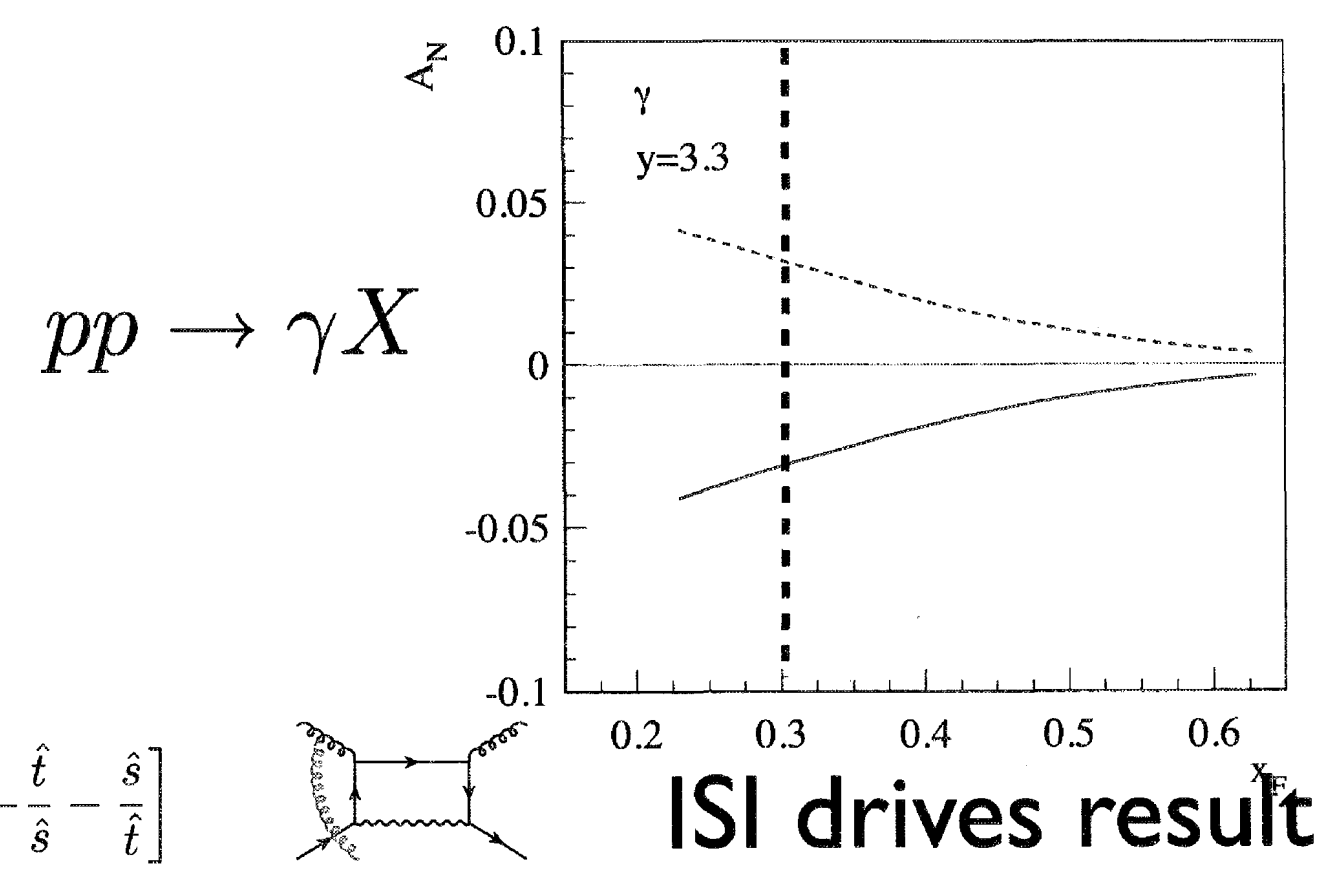

, the latest Sivers function from [2], and DSS fragmentation function [3].

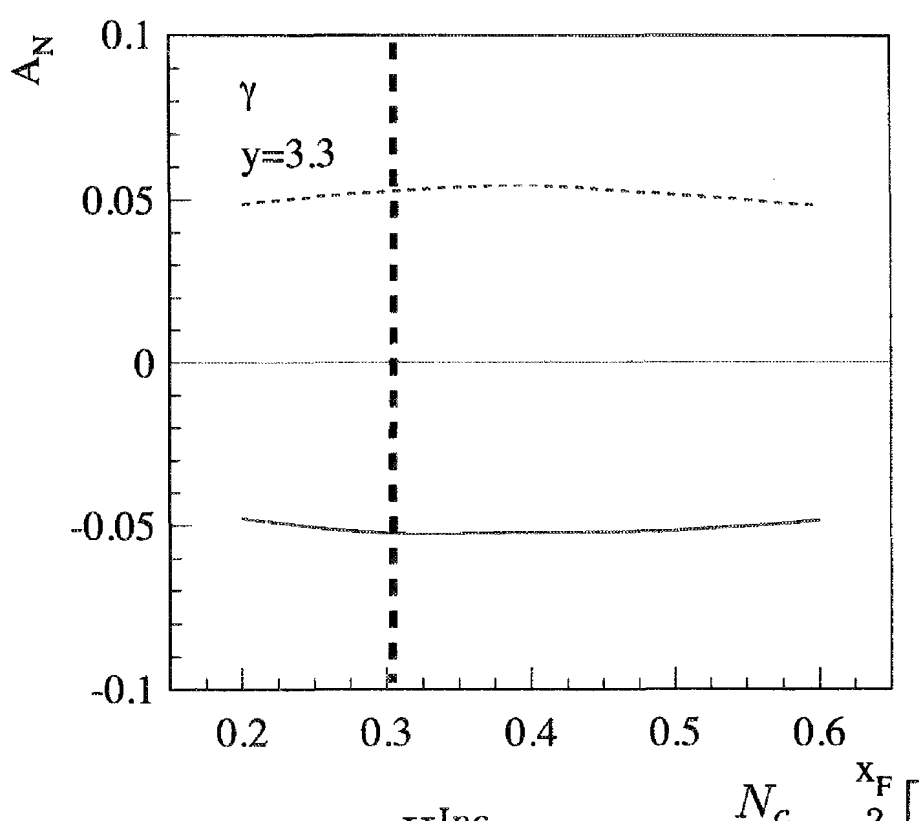

$$
H_{q g \rightarrow \gamma q}^{\mathrm{Inc}}=-\frac{N_{c}}{N_{c}^{2}-1} e_{q}^{2}\left[-\frac{\hat{t}}{\hat{s}}-\frac{\hat{s}}{\hat{t}}\right]
$$




\section{Azimuthal asymmetries for hadron distributions inside a jet in hadronic collisions}
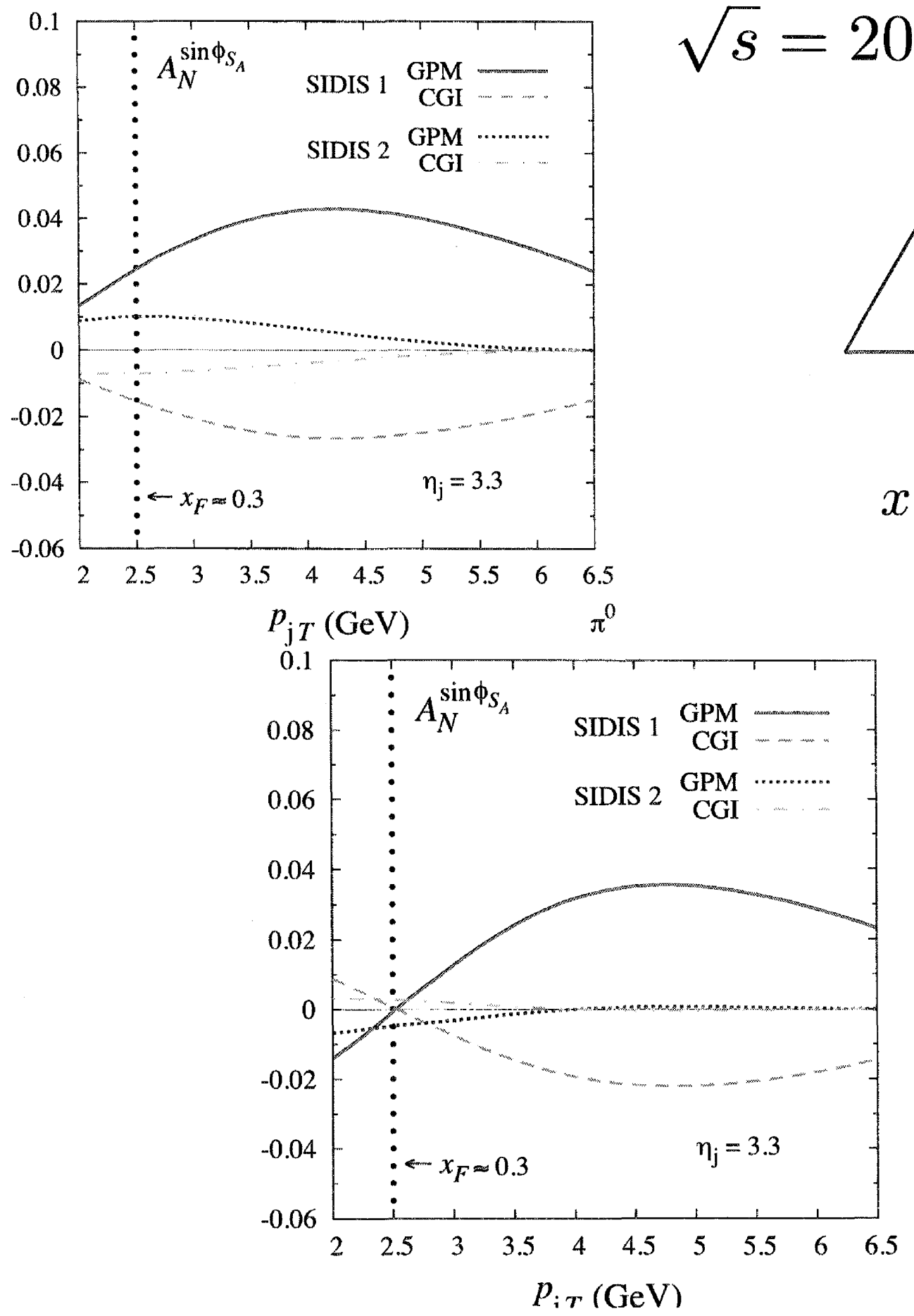

$\sqrt{s}=200 \mathrm{GeV}$

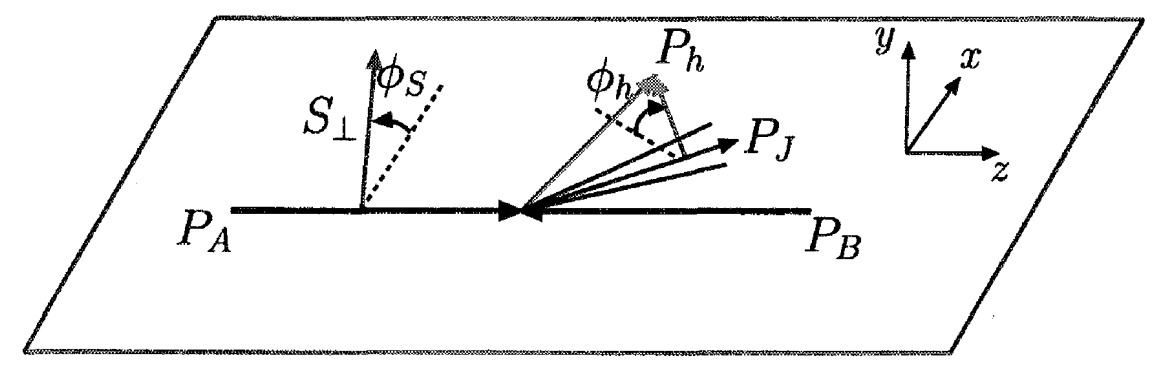

$$
x_{F}=\frac{2 P_{T}}{\sqrt{s}} \sinh (\eta)
$$

$\pi^{-}$

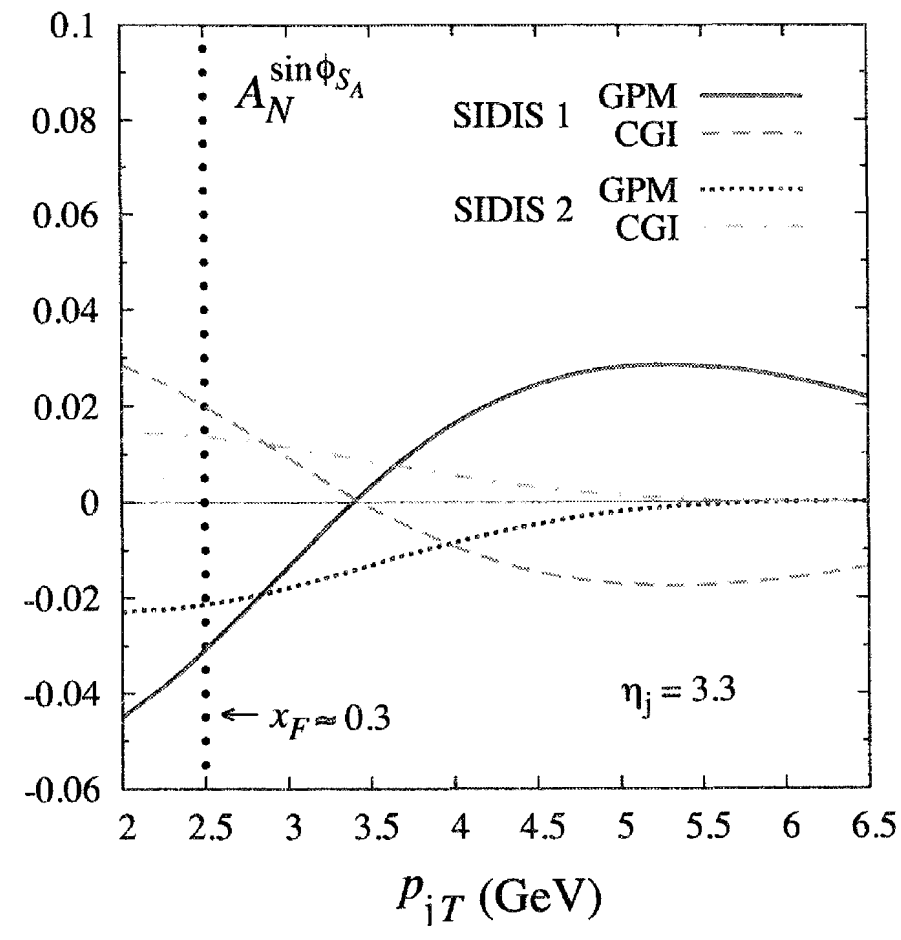


Implementing a $k_{T}$ expansion collinear twist

\section{three expression emerges}

$$
\begin{aligned}
E_{h} \frac{d \Delta \sigma}{d^{3} P_{h}}= & \frac{\alpha_{s}^{2}}{s} \sum_{a b c} \int \frac{d z_{c}}{z_{c}^{2}} D_{h / c}\left(z_{c}\right) \frac{\epsilon^{P_{h} S_{T} n \bar{n}}}{z_{c} \tilde{u}} \frac{1}{x}\left(T_{F}(x, x)-x \frac{d}{d x} T_{F}(x, x)\right) \\
& \times \int \frac{d x_{b}}{x_{b}} f_{b / B}\left(x_{b}\right) \int H_{a b \rightarrow c}^{\mathrm{Inc}}(\tilde{s}, \tilde{t}, \tilde{u}) \frac{1}{x_{b} s+T / z_{c}}
\end{aligned}
$$

?. same as Kouvaris, Qiu ,Vogelsang, and Yuan PRD 2006

- Twist 3 and twist 2 approach connection we have another term ....comes from $H_{a b \rightarrow c}^{\operatorname{Inc}}(\hat{s}, \hat{t}, \hat{u})$ 


\section{Conclusions}

- Generalize GPM w/ color--can then perform global analysis

- Elephant in the room is break down of factorization for these processes

- Appears to be connection between generalized parton model at twist 3 and twist 3 approach

- Estimate mismatch-investigating LG Z. Kang

- TMD fact. is assumed in both GPM and GGPM is this a reasonable pheno. approximation?

- Direct photon driven by same ISI factor as in DY 


\title{
Understanding forward particle production
}

\author{
Roman Pasechnik
}

Uppsala University, THEP group

In collaboration with

B. Kopeliovich

(USM, Chile) 


\section{We will talk about...}

$>$ Color neutralization and soft physics in diffractive DIS

$>$ Sudakov suppression and elastic scattering

$\overline{\underline{T}}>$ Drell-Yan at high energies: diffractive vs inclusive

$>$ Large and small dipoles

Dikonalization of the elastic amplitude and gap survival

$>$ Summary 


\section{Gap survival vs. eikonalization}

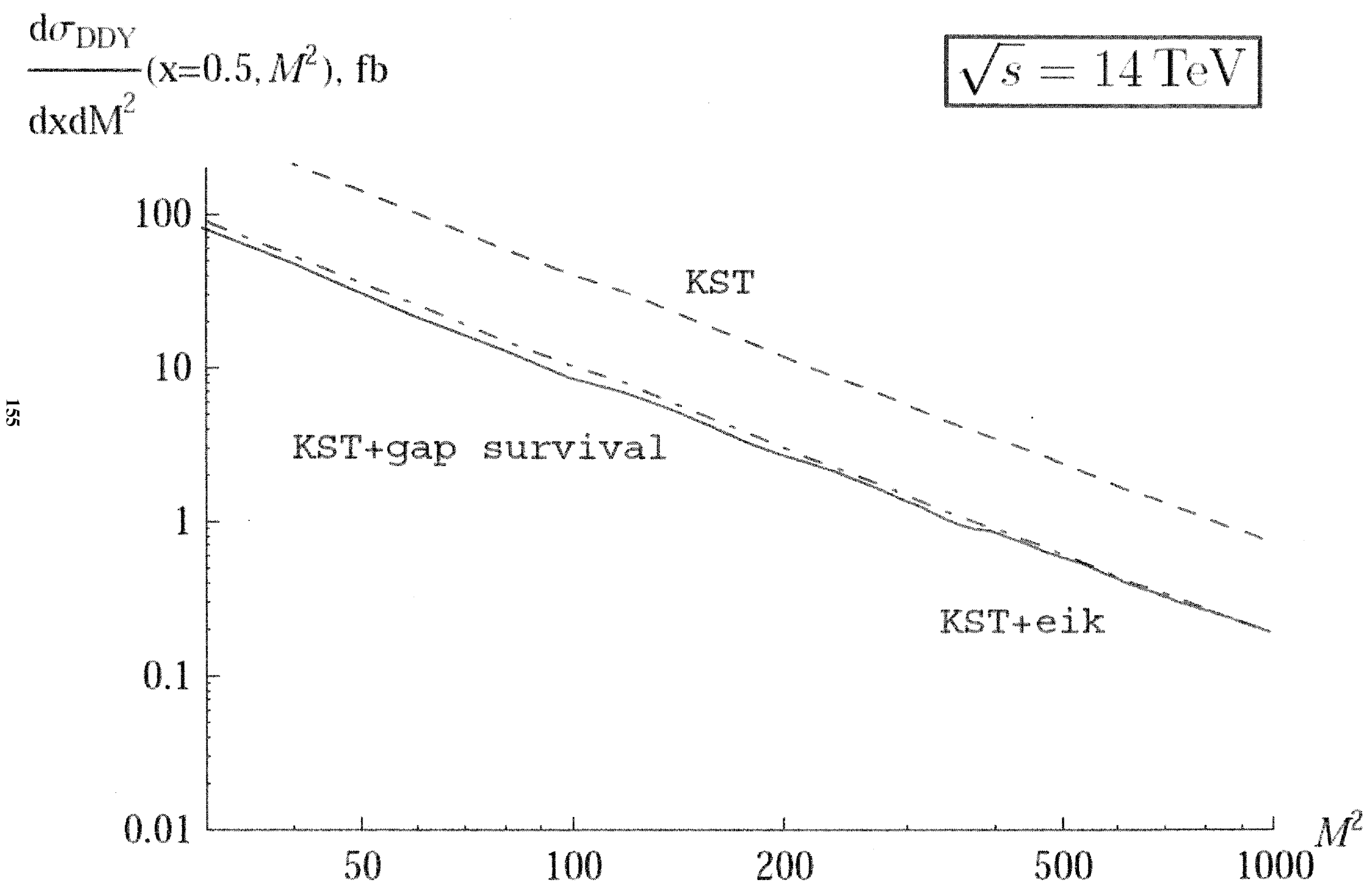




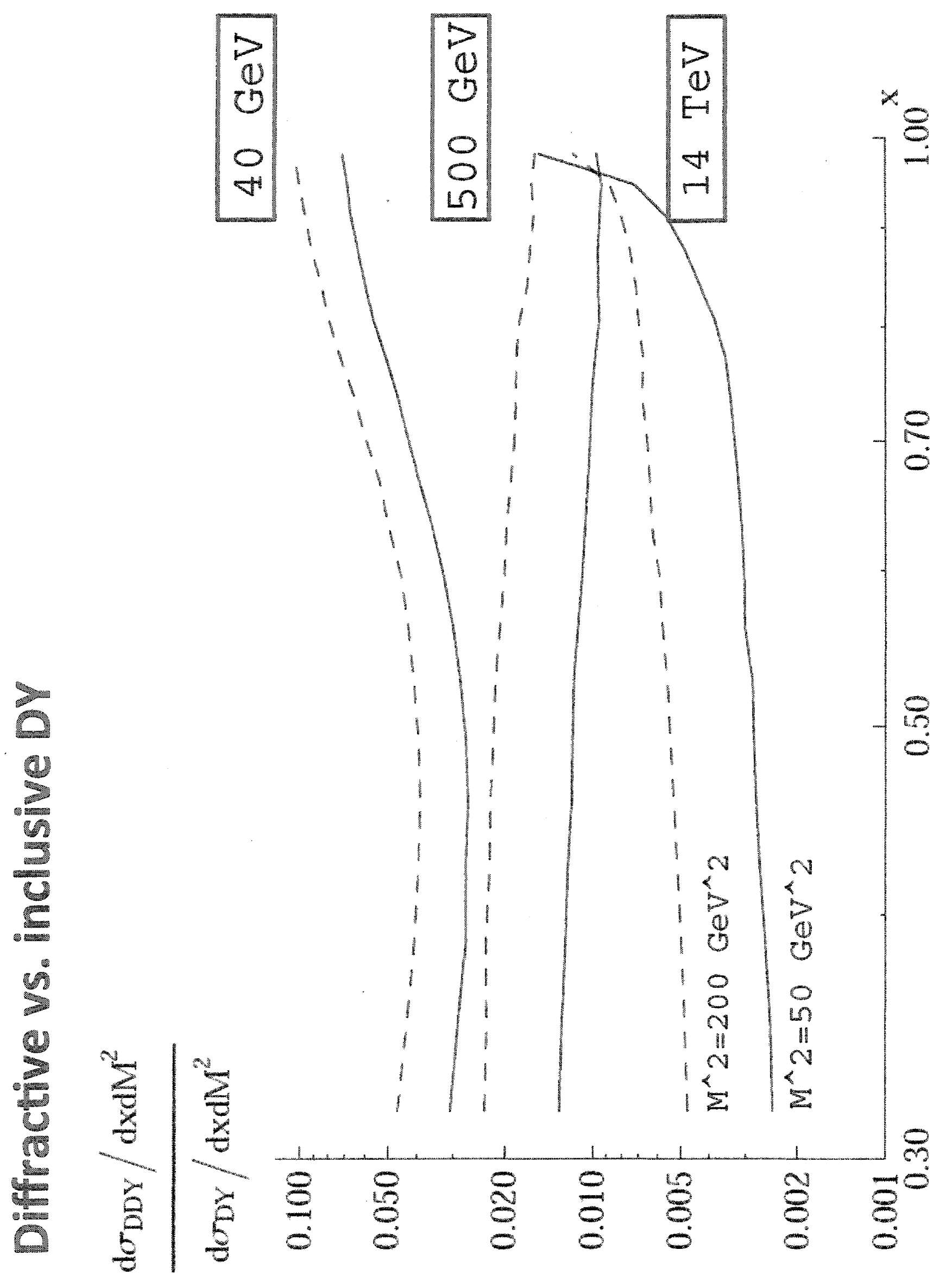




\section{Diffractive vs. inclusive DY}

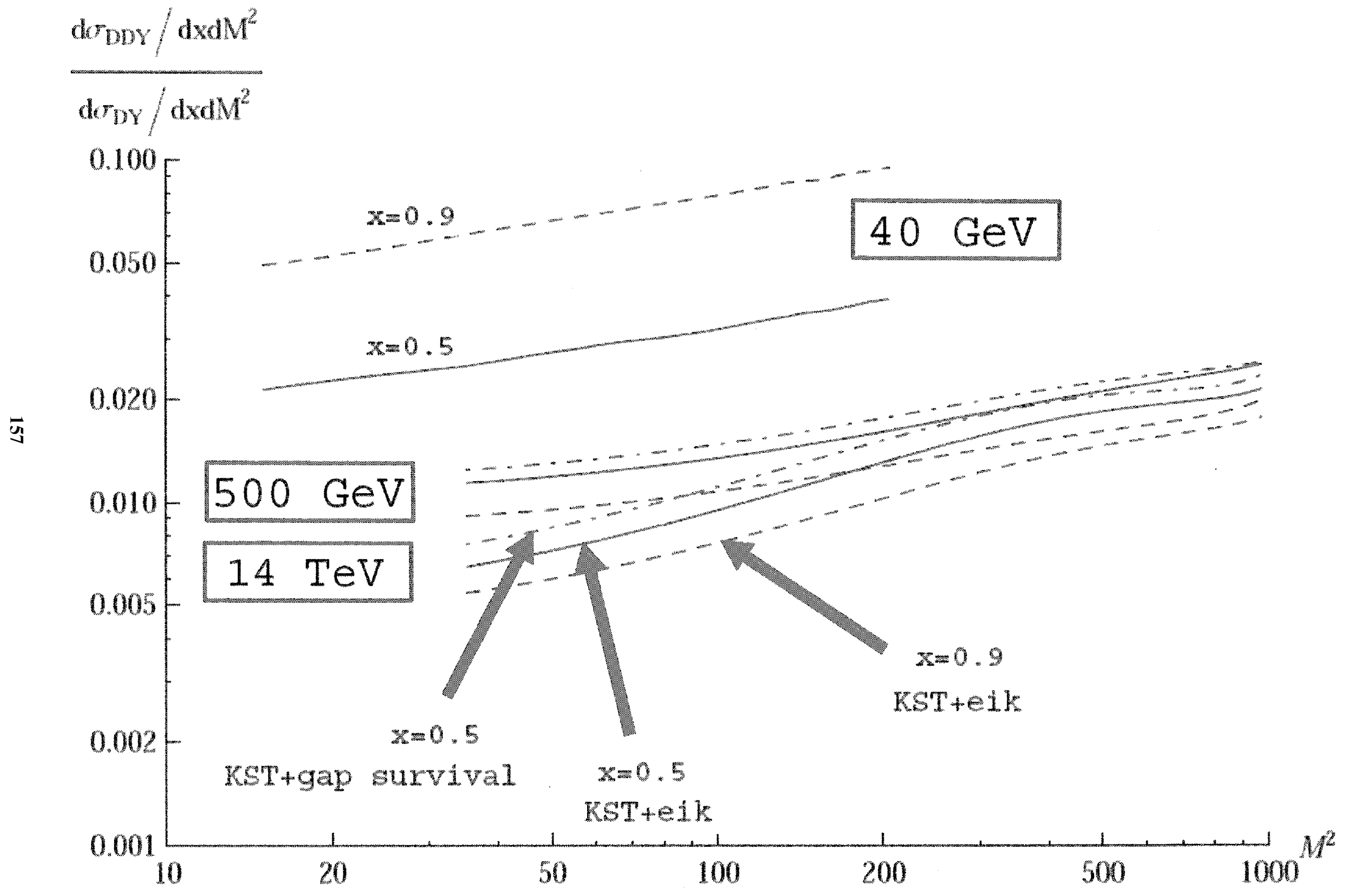




\section{Theory uncertainties}

$\mathrm{d} \sigma_{\mathrm{DDY}}$ $\frac{\mathrm{dxdM}}{2}\left(\mathrm{x}, M^{2}=50 \mathrm{GeV}^{2}\right), \mathrm{fb}$

प్

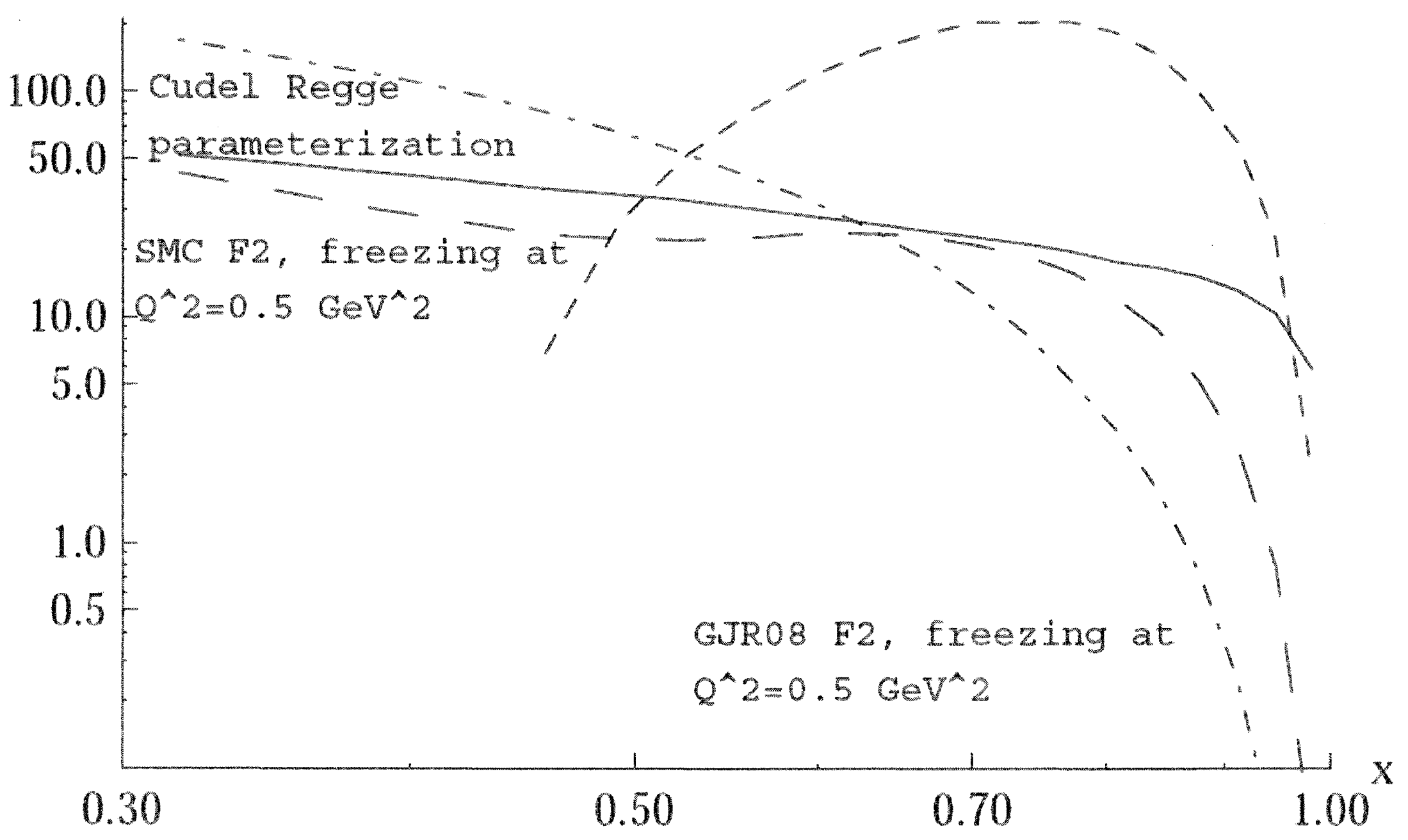




\section{Conclusions}

A quark cannot radiate photon diffractively in the forward direction

A hadron can radiate photon diffractively in the forward direction because of the transverse motion of quarks

$>$ The ratio diffractive/inclusive DY cross sections falls with energy and rises with photon dilepton mass due to the saturated shape of the dipole cross section

$>$ Hard and soft interactions contribute to the DDY on the same footing, which is the dramatic breakdown of the QCD factorisation

$>$ Main features of Drell-Yan diffraction are valid for other Abelian processes

$>$ Experimental measurements of DDY would allow to probe directly the dipole cross section at large separations, as well as the proton structure function at soft and semihard scales, and large $x$

$>$ DDY is a good playground for diffractive production of heavy flavors 


\title{
Drell-Yan at forward rapidities
}

\author{
Anna M. Staśto
}

May 16, 2011

We analyze the Drell-Yan lepton pair production at forward rapidity at the Large Hadron Collider. Using the dipole framework for the computation of the cross section we find a a significant suppression in comparison to the collinear factorization formula due to saturation effects in the dipole cross section. We develop a twist expansion in powers of $Q_{s}\left(x_{2}\right) / M$ where $Q_{s}$ is the saturation scale and $M$ the invariant mass of the produced lepton pair. For the nominal LHC energy the leading twist description is sufficient down to masses of $6 \mathrm{GeV}$. Below that value the higher twist terms give a significant contribution. We perform the analysis for Tevatron and LHC energies.

In collaboration with $K$. Golec-Biermat and E.Lewandowska 


\section{Dipole model for Drell-Yan}

Drell-Yan in the dipole model at small $x$

$$
\begin{gathered}
\frac{d^{2} \sigma_{T, L}^{D Y}}{d M^{2} d x_{F}}=\frac{\alpha_{e m}}{6 \pi M^{2}} \frac{1}{x_{1}+x_{2}} \sum_{f} \int_{x_{1}}^{1} \frac{d z}{z} F_{2}^{f}\left(\frac{x_{1}}{z}, M^{2}\right) \sigma_{T, L}^{f}\left(q p \rightarrow \gamma^{*} X\right) . \\
\begin{array}{c}
\text { Structure function of the } \\
\text { incoming projectile }
\end{array} \quad z \quad \begin{array}{c}
\text { Fraction of the energy of the } \\
\text { quark taken by the photon }
\end{array}
\end{gathered}
$$

Radiation of the photon from the fast quark

$$
\begin{gathered}
\sigma_{T, L}^{f}\left(q p \rightarrow \gamma^{*} X\right)=\int d^{2} r W_{T, L}^{f}\left(z, r, M^{2}, m_{f}\right) \sigma_{q q}\left(x_{2}, z r\right), \\
W_{T}^{f}=\frac{\alpha_{e m}}{\pi^{2}}\left\{\left[1+(1-z)^{2}\right] \eta^{2} K_{1}^{2}(\eta r)+m_{f}^{2} z^{4} K_{0}^{2}(\eta r)\right\} \\
W_{L}^{f}=\frac{2 \alpha_{e m}}{\pi^{2}} M^{2}(1-z)^{2} K_{0}^{2}(\eta r)
\end{gathered}
$$

$r \quad$ Photon-quark transverse separation

$$
\eta^{2}=(1-z) M^{2}+z^{2} m_{f}^{2}
$$

As an example use the Golec Biernat and Wusthoff formula

$$
\sigma_{q q}(x, r)=\sigma_{0}\left\{1-\exp \left(-r^{2} Q_{s}^{2}(x) / 4\right)\right\} .
$$




\section{Predictions for LHC}

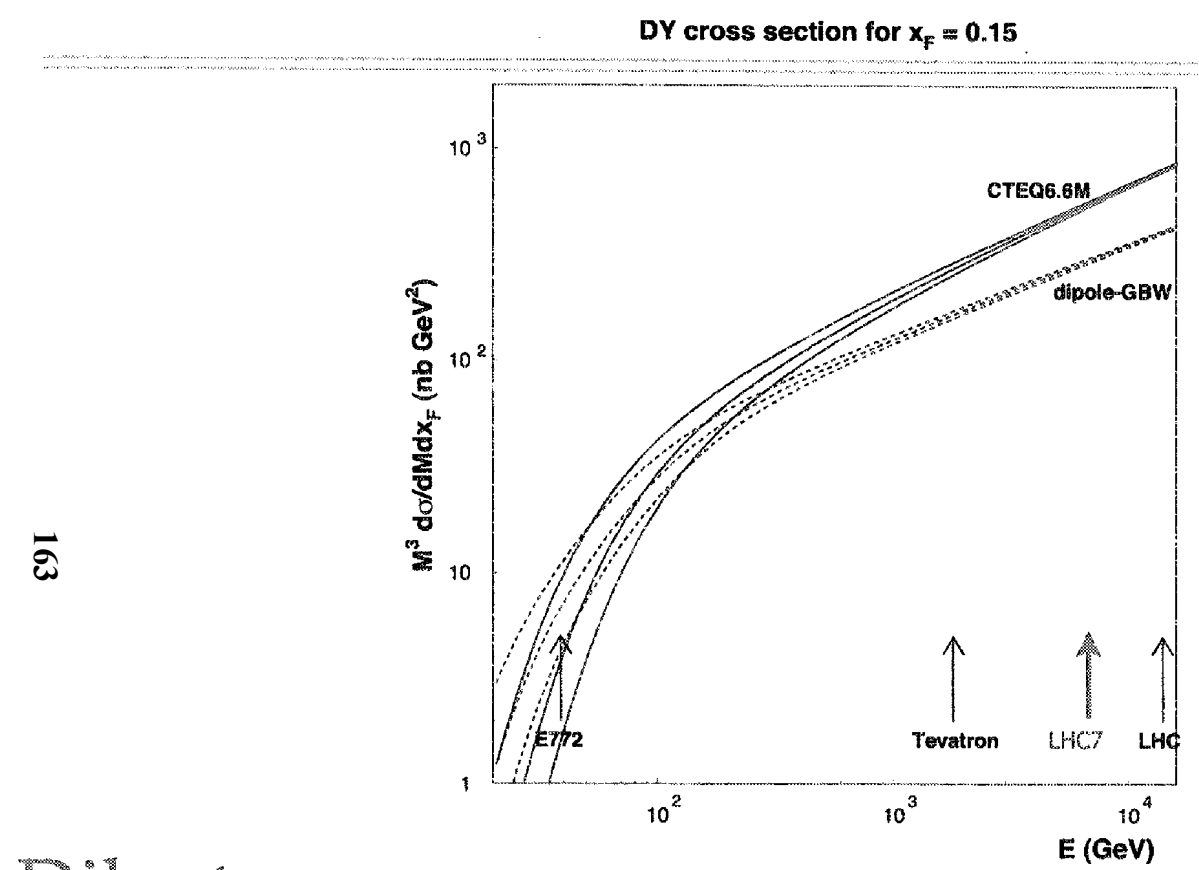

Dilepton mass $M=6,8,10 \mathrm{GeV}$

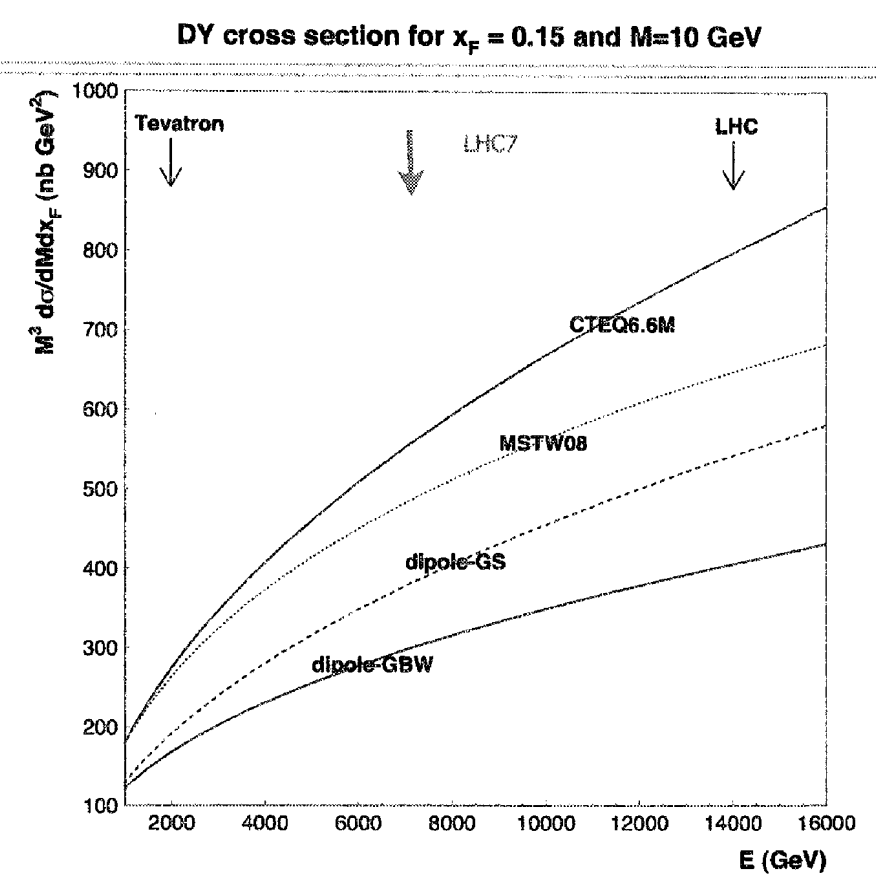

dipole-GS (Golec-Sapeta) DGLAP included

Large differences between collinear approaches $x_{2} \simeq 3 \cdot 10^{-6}-10^{-5}$ typical values probed at energies $14-7 \mathrm{TeV}$

$$
y \sim 5-6 \quad \text { range of rapidities }
$$

Dipole predictions systematically lower than the collinear calculations. 


\section{Twist expansion for Drell- Yan}

It is more complicated than in DIS, because of the convolution with the structure function of the forward projectile.

$\bar{a}$

$$
\frac{d^{2} \sigma_{T}^{D Y}}{d M^{2} d x_{F}}=\frac{\alpha_{e m}^{2} \sigma_{0}}{6 \pi^{2} M^{2}} \frac{1}{x_{1}+x_{2}} \int_{c-i \infty}^{c+i \infty} \frac{d \gamma}{2 \pi i} G(\gamma) \widetilde{H}_{T}(\gamma)\left(\frac{Q_{s}^{2}\left(x_{2}\right)}{4 M^{2}}\right)^{\gamma}
$$

$$
\times \int_{x_{1}}^{1} \frac{d z}{z} F_{2}\left(\frac{x_{1}}{z}, M^{2}\right)\left[1+(1-z)^{2}\right]\left(\frac{z^{2}}{1-z}\right)^{\gamma}
$$

Cannot directly perform integral over $\mathrm{z}$ (fraction of the lightcone momentum of the initial quark carried away by the photon), since it is weighted by the structure function of the projectile.

Two methods: fully analytical in terms of expansion in (1-x1). Semi-analytical with exact results for twist contributions 


\section{Twist expansion: explicit}

Twist 2: contribution from $\quad \gamma=1$

$$
\frac{d^{2} \sigma_{T}^{D Y(\tau=2)}}{d M^{2} d x_{F}}=\Delta_{T, 2}^{(0)}+\Delta_{T, 2}^{(k>0)}
$$

宛

$$
\begin{aligned}
\Delta_{T, 2}^{(0)} & =\frac{\alpha_{e m}^{2} \sigma_{0} F_{2}\left(x_{1}, I^{2}\right)}{6 \pi^{2} M^{2}} \times 2 \frac{Q_{s}^{2}\left(x_{2}\right)}{4 M^{2}}\left[\frac{4}{3} \gamma_{E}-1+\frac{2}{3} \psi\left(\frac{5}{2}\right)-\frac{2}{3} \ln \frac{Q_{s}^{2}\left(x_{2}\right)}{4 M^{2}\left(1-x_{1}\right)}\right] \\
\Delta_{T, 2}^{(k>0)} & =\frac{\alpha_{e m}^{2} \sigma_{0}}{6 \pi^{2} M^{2}} \frac{1}{x_{1}+x_{2}} \times \frac{4}{3}\left(\frac{Q_{s}^{2}\left(x_{2}\right)}{4 M^{2}}\right) \int_{x_{1}}^{1} d z \frac{2 F_{2}\left(\frac{x_{1}}{3} \mid I^{2}\right)\left(1+(1-)^{2}\right)-F_{2}\left(x_{7}, I^{2}\right)}{1-z}
\end{aligned}
$$

First term contains the contribution from the double pole in the Mellin space (hence the logarithm). The result is exact twist 2 contribution.

Note the integrals over $\mathrm{z}$ over the structure function of the projectile. 


\section{Twist expansion for DY: results}

DY cross sections for $x_{F}=0.15$

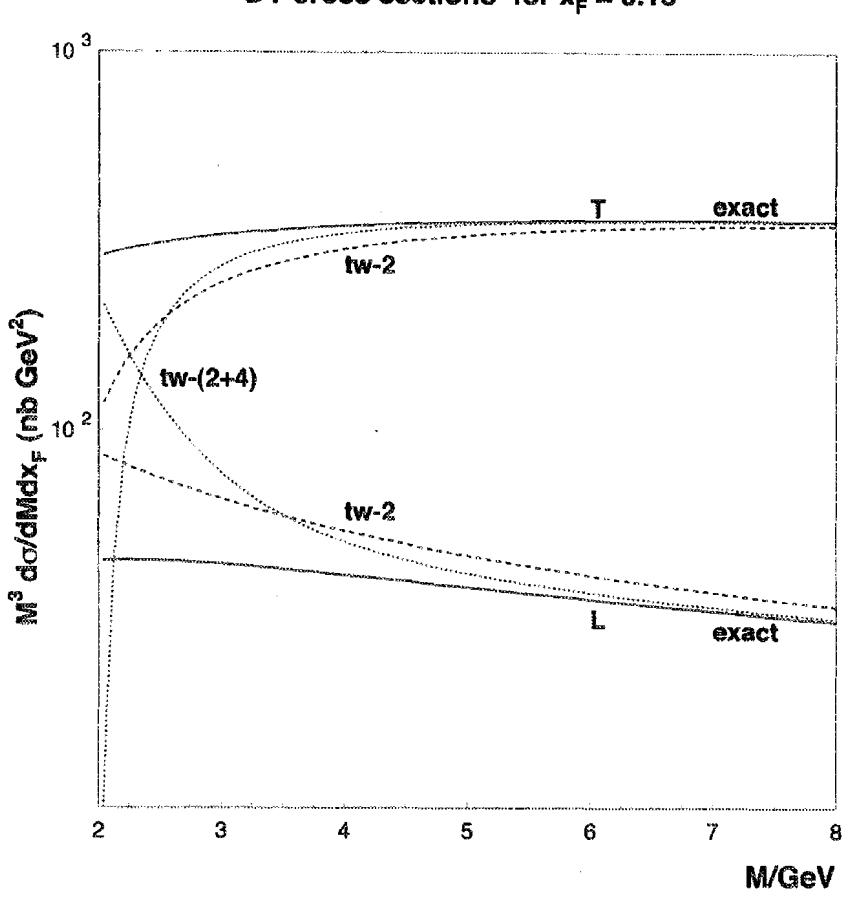

DY cross sections for $x_{F}=0.15$

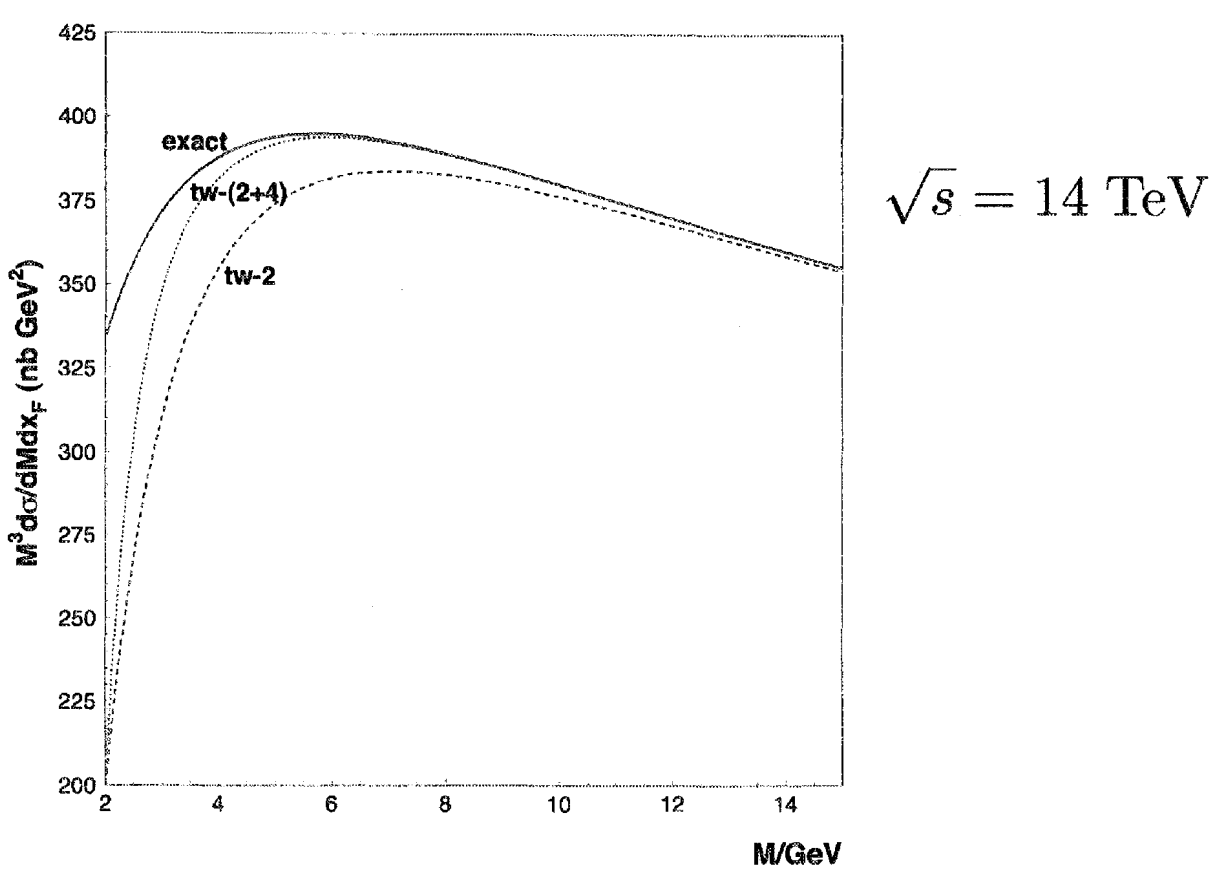

- Twist expansion divergent for $M<4$.

- For higher masses $M>6$ twist 2 sufficient.

- For longitudinal twist 2 overestimates, for transverse part underestimates the exact result.

- The sum is better approximated by twist expansion. 


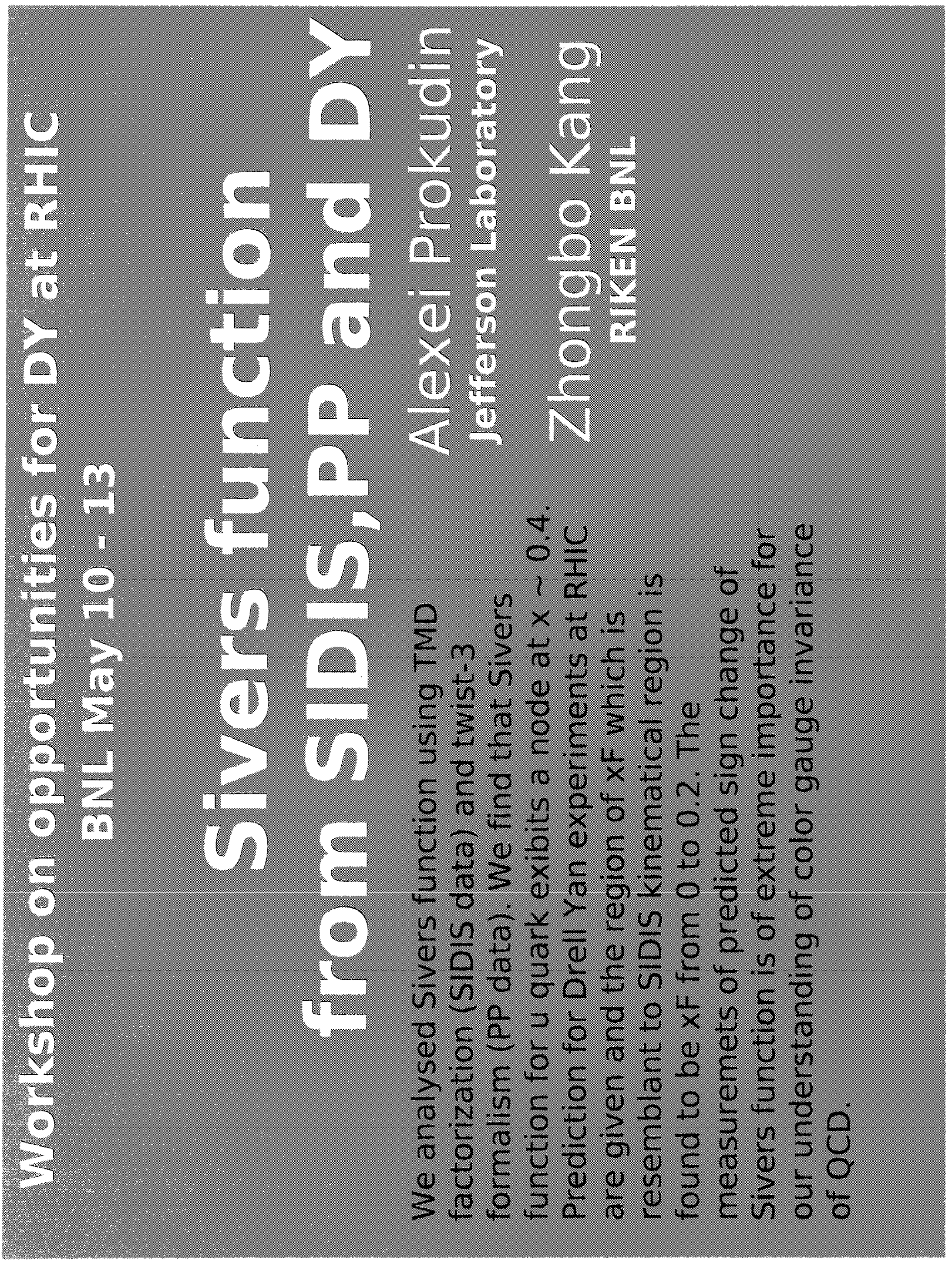




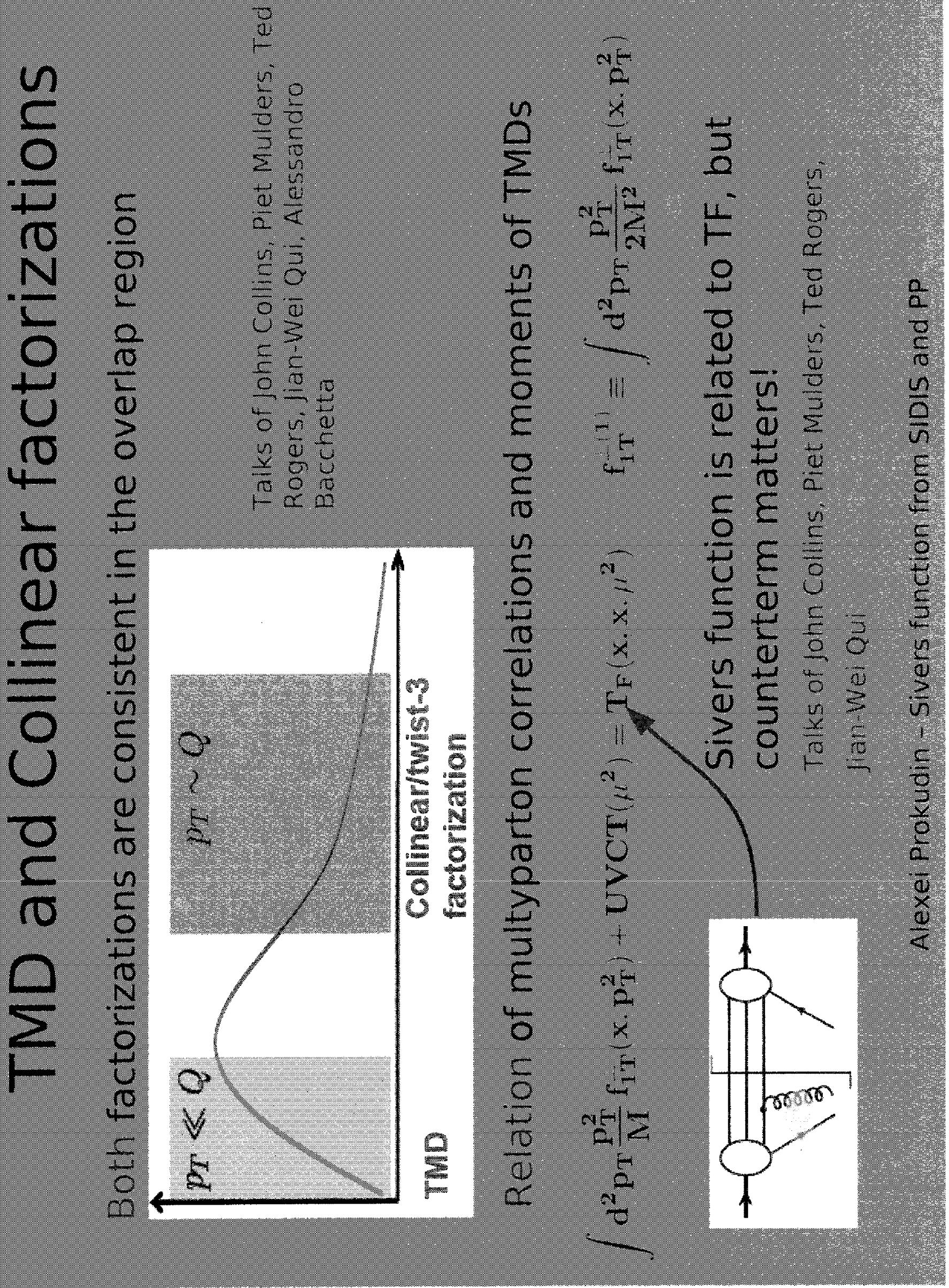




\section{Proton Proton}

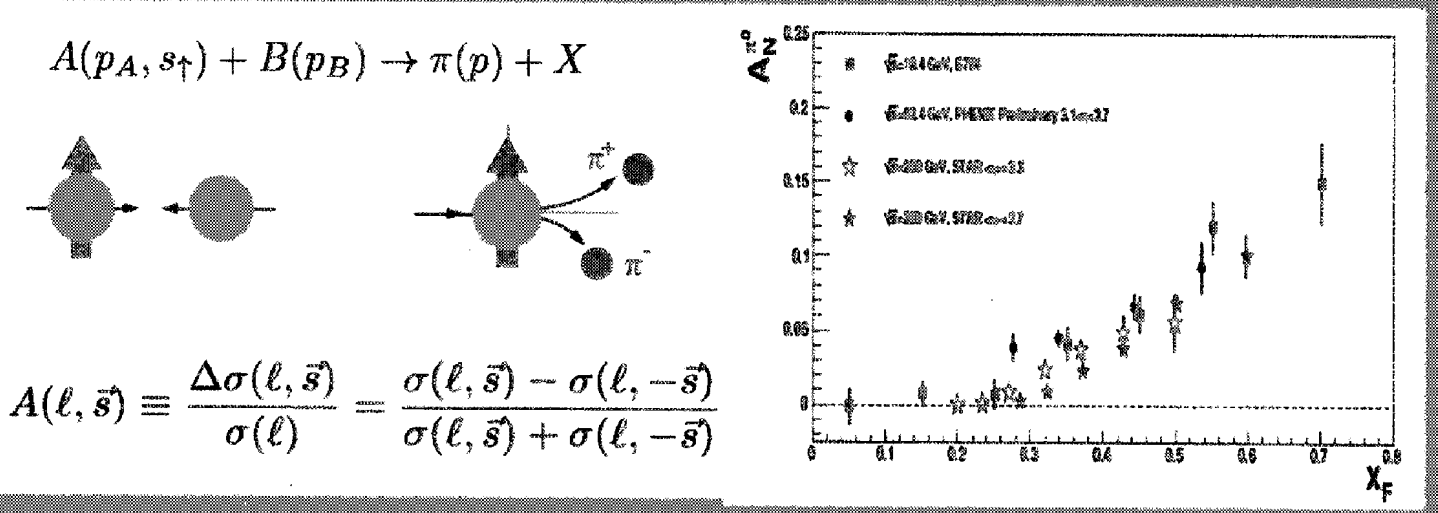

$\vec{\sigma}$

SIDIS

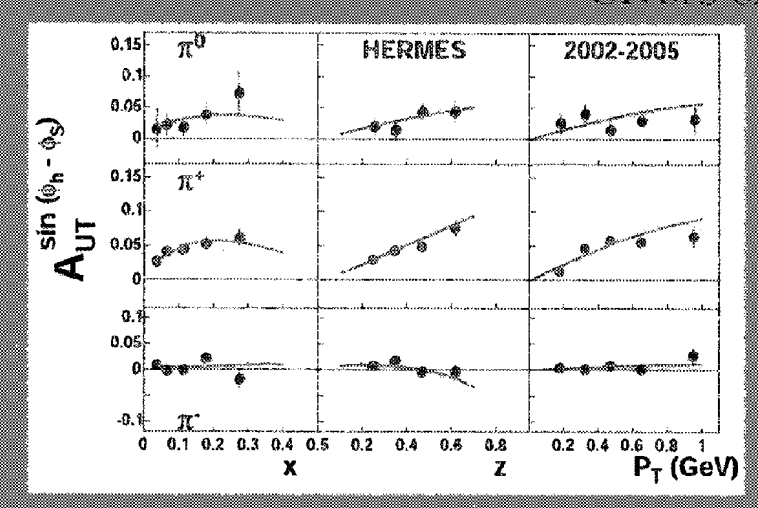

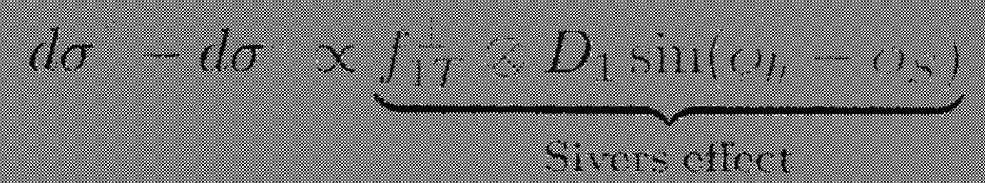

Only one scale $P_{T}$ Collinear analysis:

Kouvaris, Qiu,

Vogelsang, Yuan (2006)

Kanazava, Koike (2010)

TMD analysis:

Anselmino et al (2006)

Alexei Prokudin - Sivers function from SIDIS and PP 


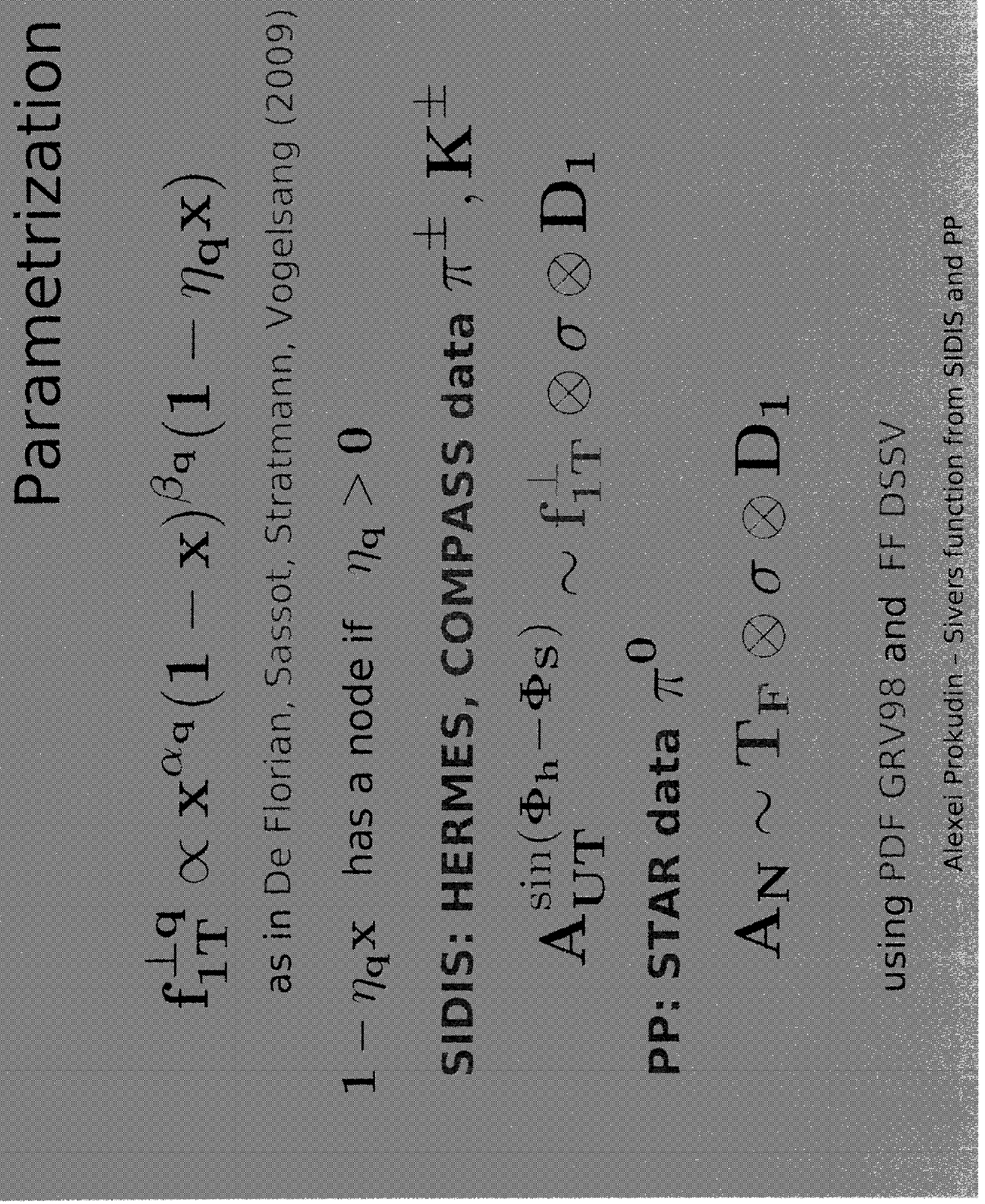




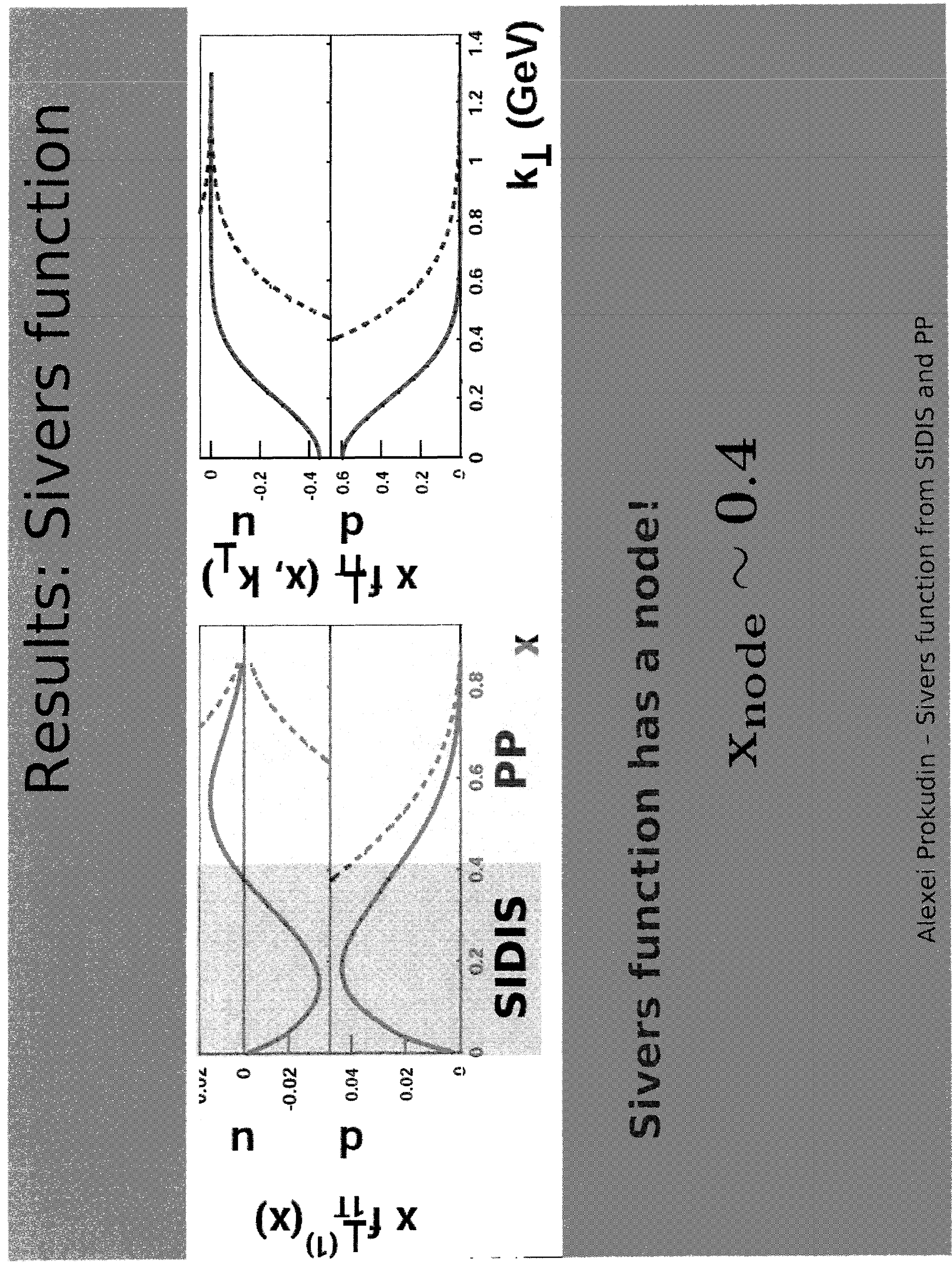




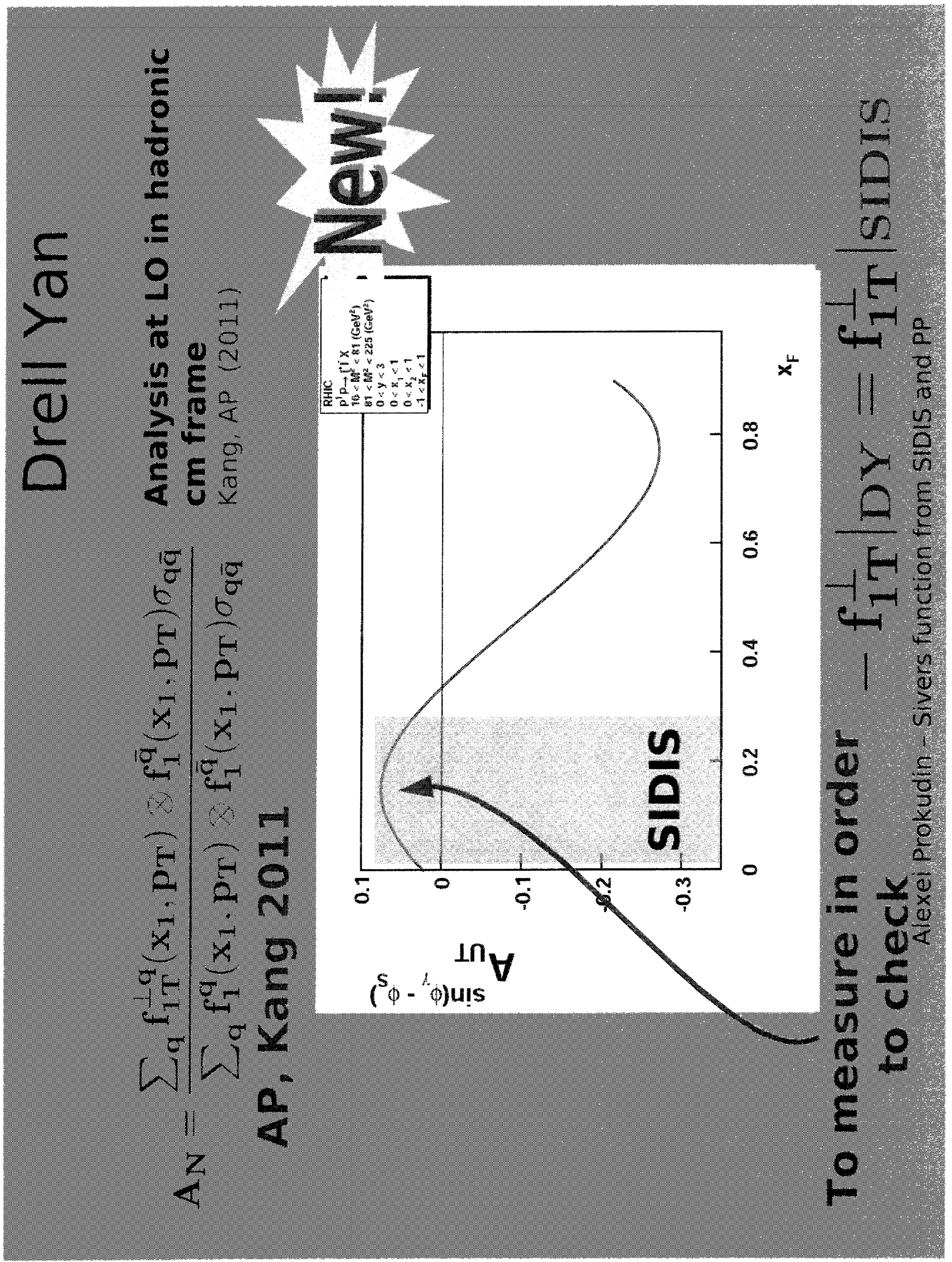


Alessandro Bacchetta

Università di Pavia and INFN Pavia

\section{TRANSVERSE PHYSICS WITH SIDIS, $e^{+} e^{-} \& p p$}

Two different topics are discussed:

1. TMD opportunities in Drell-Yan

2. Accessing transversity with dihadron fragmentation functions

Concerning the first topic, the relevance of the sign change of the Sivers function is discussed. A simple implementation of neural-network fits is discussed as a method to critically assess the current knowiedge of the Sivers function, with particular emphasis on the presence of poles.

A few other crucial questions on TMDs that can be addressed in Drell-Yan experiments are mentioned: the knowledge of unpolarized TMDs, their $x$ dependence, their transversemomentum shape, and their flavor dependence.

Concerning the second topic, the results of the first extraction of the transversity distribution function based on collinear factorization is discussed. The results are derived from experimental measurements in SIDIS and annihilation. A comparison with the transversity extracted in TMD factorization is shown. 


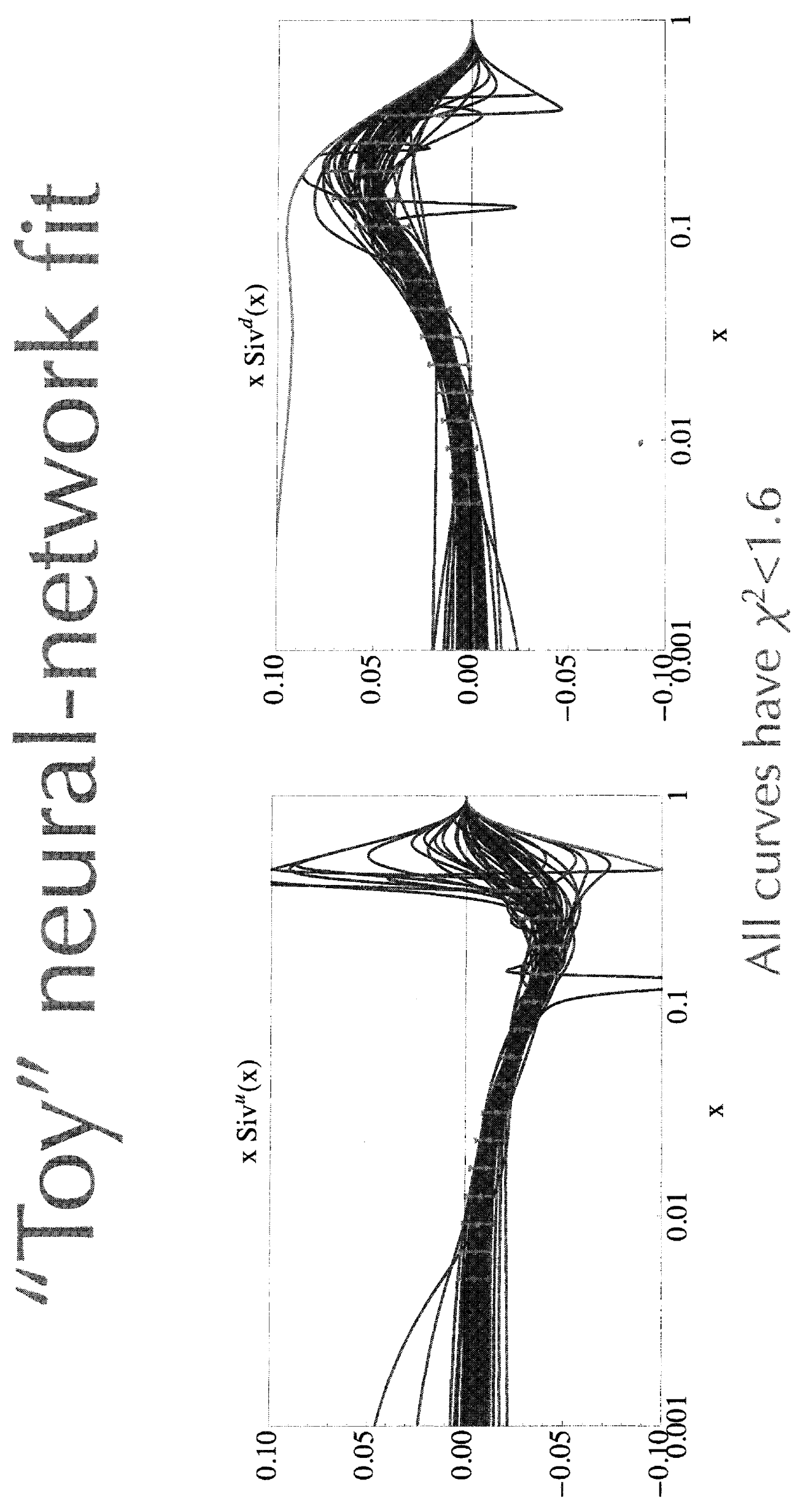




\section{"Toy" neural-network fit}
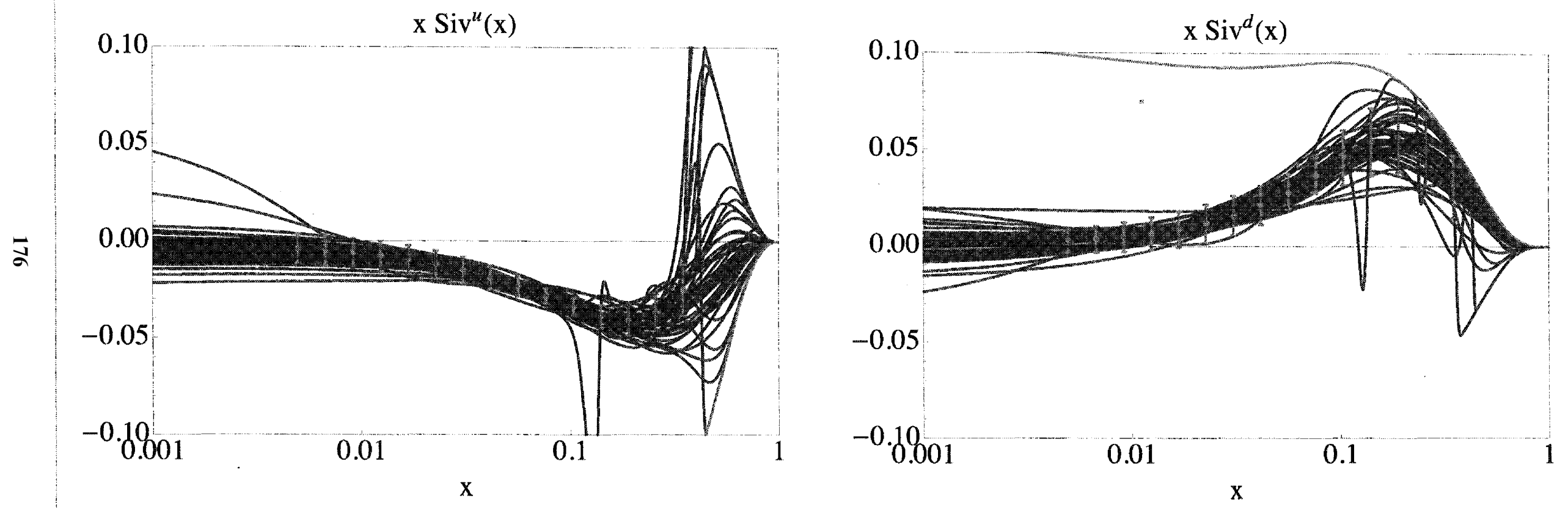

All curves have $\chi^{2}<1.6$

Neural-network fits are the best tools to avoid biased functional forms 


\section{BLNY fit}

Landry, Brock, Nadolsky, Yuan, PRD67 (03)

\begin{tabular}{|c|c|c|c|c|}
\hline Experiment & Reference & Reaction & $\sqrt{S}(\mathrm{GeV})$ & $\delta N_{\text {exp }}$ \\
\hline \hline R209 & {$[14]$} & $p+p \rightarrow \mu^{+} \mu^{-}+X$ & 62 & $10 \%$ \\
\hline E605 & {$[15]$} & $p+C u \rightarrow \mu^{+} \mu^{-}+X$ & 38.8 & $15 \%$ \\
\hline E288 & {$[16]$} & $p+C u \rightarrow \mu^{+} \mu^{-}+X$ & 27.4 & $25 \%$ \\
\hline $\begin{array}{c}\text { CDF- } Z \\
\text { (Run-0) }\end{array}$ & {$[17]$} & $p+\bar{p} \rightarrow Z+X$ & 1800 & - \\
\hline $\begin{array}{c}\text { DØ - } Z \\
\text { (Run-1) }\end{array}$ & {$[18]$} & $p+\bar{p} \rightarrow Z+X$ & 1800 & $4.3 \%$ \\
\hline $\begin{array}{c}\text { CDF- } Z \\
\text { (Run-1) }\end{array}$ & {$[19]$} & $p+\bar{p} \rightarrow Z+X$ & 1800 & $3.9 \%$ \\
\hline
\end{tabular}




\section{BLNY fit}

Landry, Brock, Nadolsky, Yuan, PRD67 (03)

$\bar{\infty}$

\begin{tabular}{|c|c|c|c|c|}
\hline Experiment & Reference & Reaction & $\sqrt{S}(\mathrm{GeV})$ & $\delta N_{\text {exp }}$ \\
\hline \hline R209 & {$[14]$} & $p+p \rightarrow \mu^{+} \mu^{-}+X$ & 62 & $10 \%$ \\
\hline E605 & {$[15]$} & $p+C u \rightarrow \mu^{+} \mu^{-}+X$ & 38.8 & $15 \%$ \\
\hline E288 & {$[16]$} & $p+C u \rightarrow \mu^{+} \mu^{-}+X$ & 27.4 & $25 \%$ \\
\hline $\begin{array}{c}\text { CDF- } Z \\
(\text { Run-0) }\end{array}$ & {$[17]$} & $p+\bar{p} \rightarrow Z+X$ & 1800 & - \\
\hline $\begin{array}{c}\text { DØ }-Z \\
(\text { Run-1) }\end{array}$ & {$[18]$} & $p+\bar{p} \rightarrow Z+X$ & 1800 & $4.3 \%$ \\
\hline $\begin{array}{c}\text { CDF-Z } \\
\text { (Run-1) }\end{array}$ & {$[19]$} & $p+\bar{p} \rightarrow Z+X$ & 1800 & $3.9 \%$ \\
\hline
\end{tabular}

$D-Y$ (including $Z$ production) is the most important source of information for unpolarized TMDs 


\section{BLNY fit}

Landry, Brock, Nadolsky, Yuan, PRD67 (03)

ปั

\begin{tabular}{|c|c|c|c|c|}
\hline Experiment & Reference & Reaction & $\sqrt{S}(\mathrm{GeV})$ & $\delta N_{\exp }$ \\
\hline $\mathrm{R} 209$ & [14] & $p+p \rightarrow \mu^{+} \mu^{-}+X$ & 62 & $10 \%$ \\
\hline E605 & [15] & $p+C u \rightarrow \mu^{+} \mu^{-}+X$ & 38.8 & $15 \%$ \\
\hline E288 & {$[16]$} & $p+C u \rightarrow \mu^{+} \mu^{-}+X$ & 27.4 & \\
\hline $\begin{array}{l}\text { CDF- } Z \\
\text { (Run-0) }\end{array}$ & {$[17]$} & $p+\bar{p} \rightarrow Z+X$ & 1800 & \\
\hline $\begin{array}{l}\mathrm{D} \varnothing-Z \\
(\mathrm{Run}-1)\end{array}$ & [18] & $p+\bar{p} \rightarrow Z+X$ & 1800 & $4.3 \%$ \\
\hline $\begin{array}{l}\text { CDF-Z } \\
\text { (Run-1) }\end{array}$ & {$[19]$} & $p+\vec{p} \rightarrow Z+X$ & 1800 & $3.9 \%$ \\
\hline
\end{tabular}

D-Y (including Z production) is the most important source of information for unpolarized TMDs 


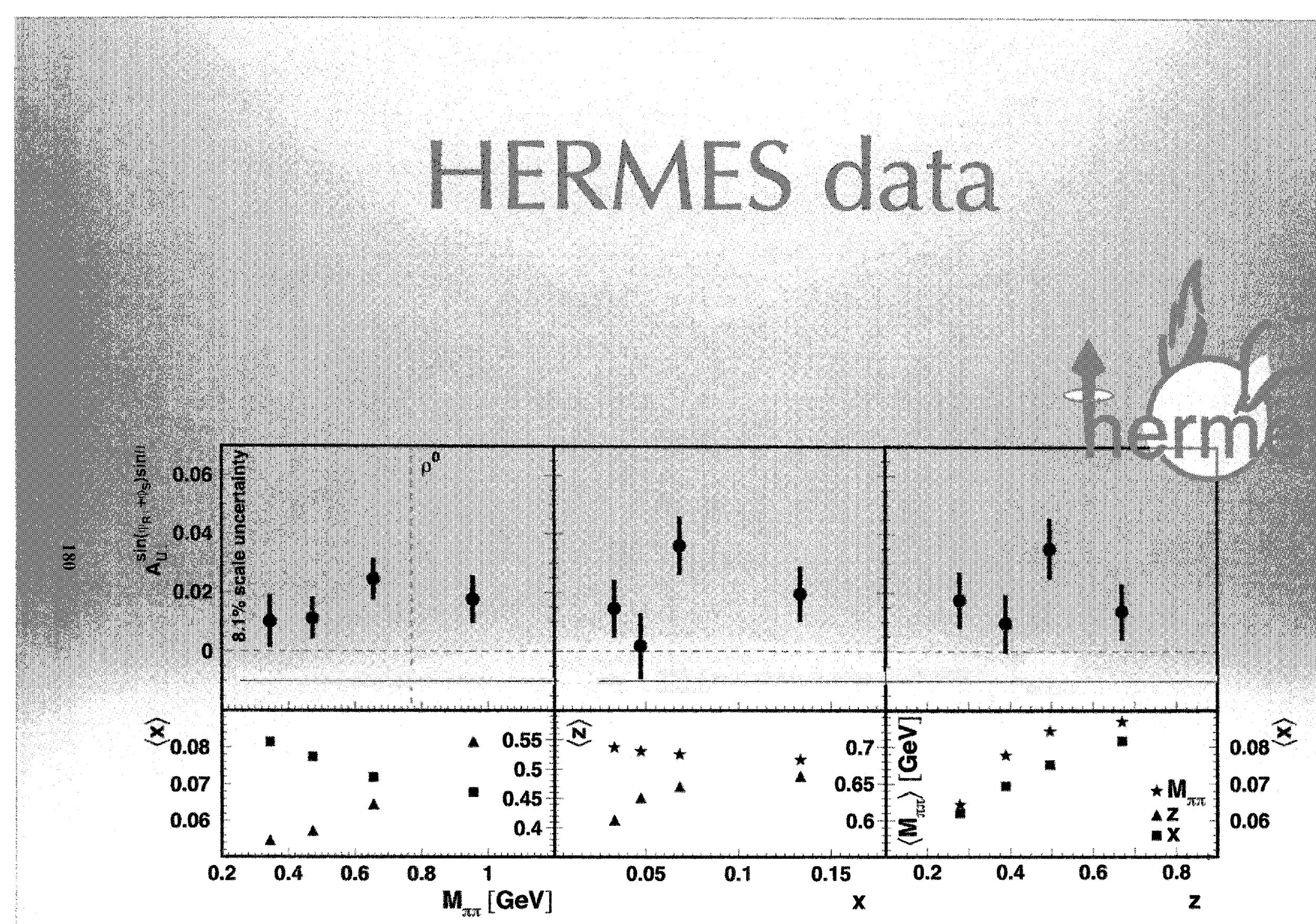




\section{HERMES data}

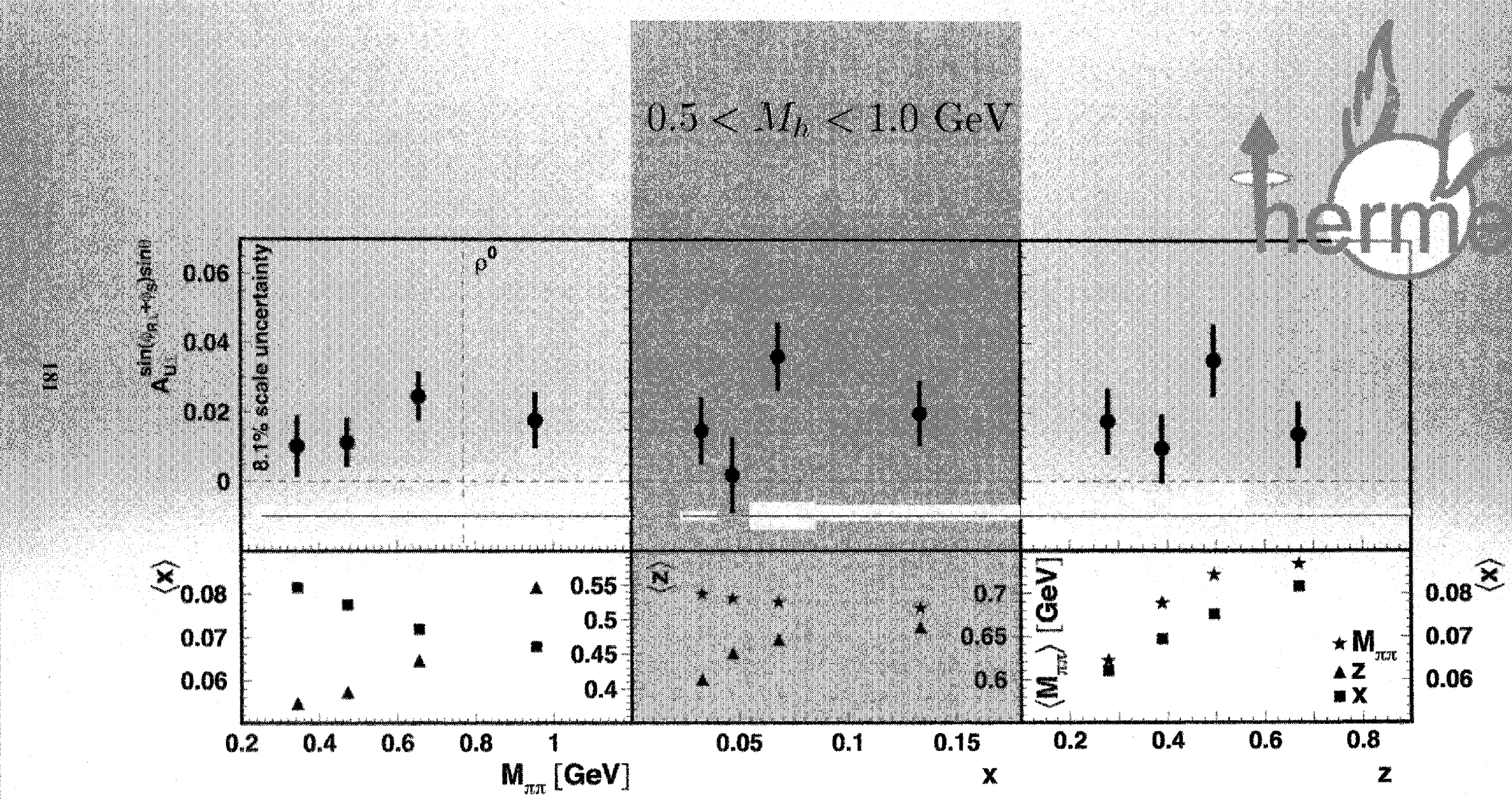




\section{BELLE data}
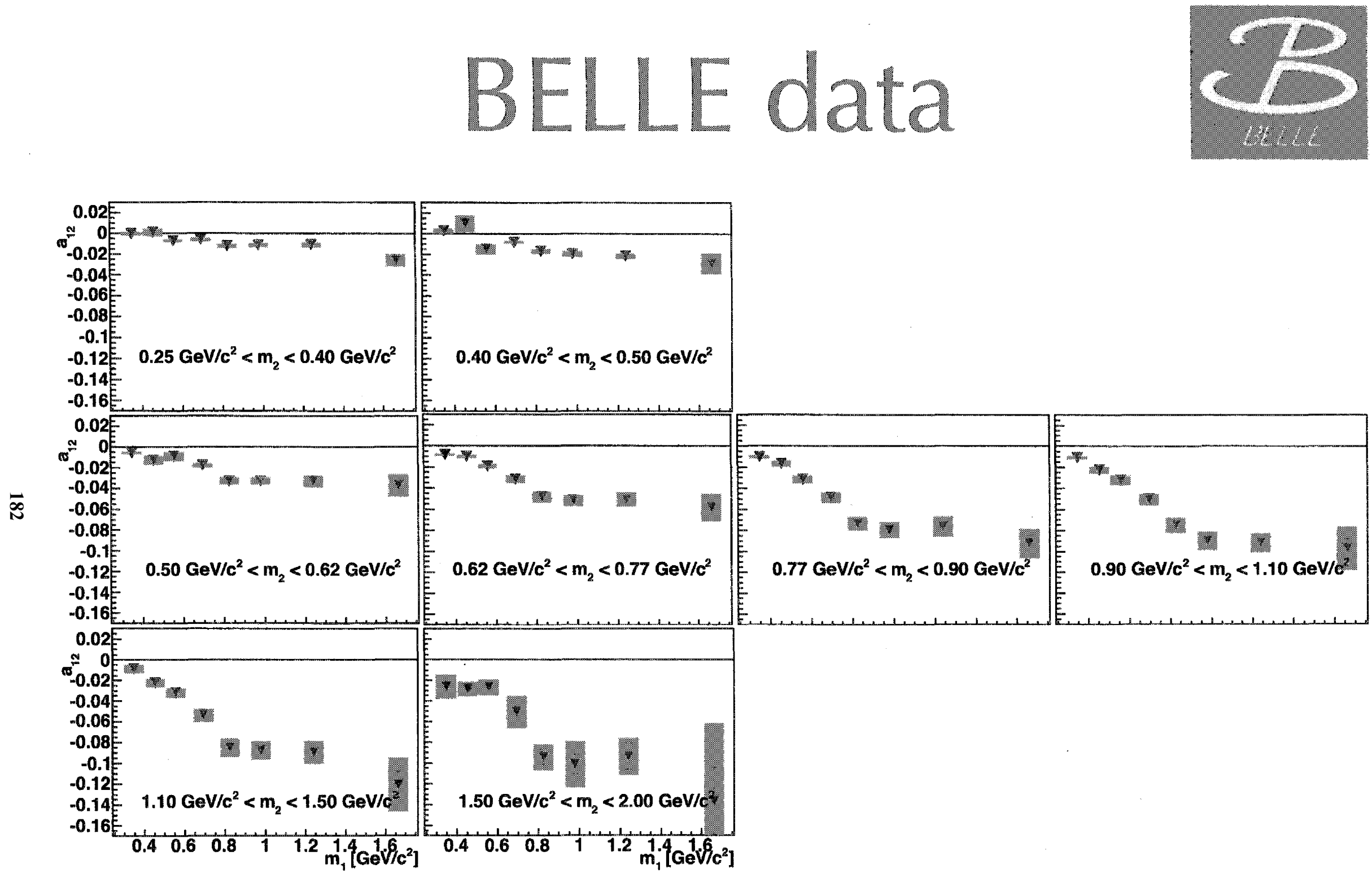

Vossen, Seidl et al. (Belle), arXiv:1104.2425 [hep-ex] 


\section{BELLE data}

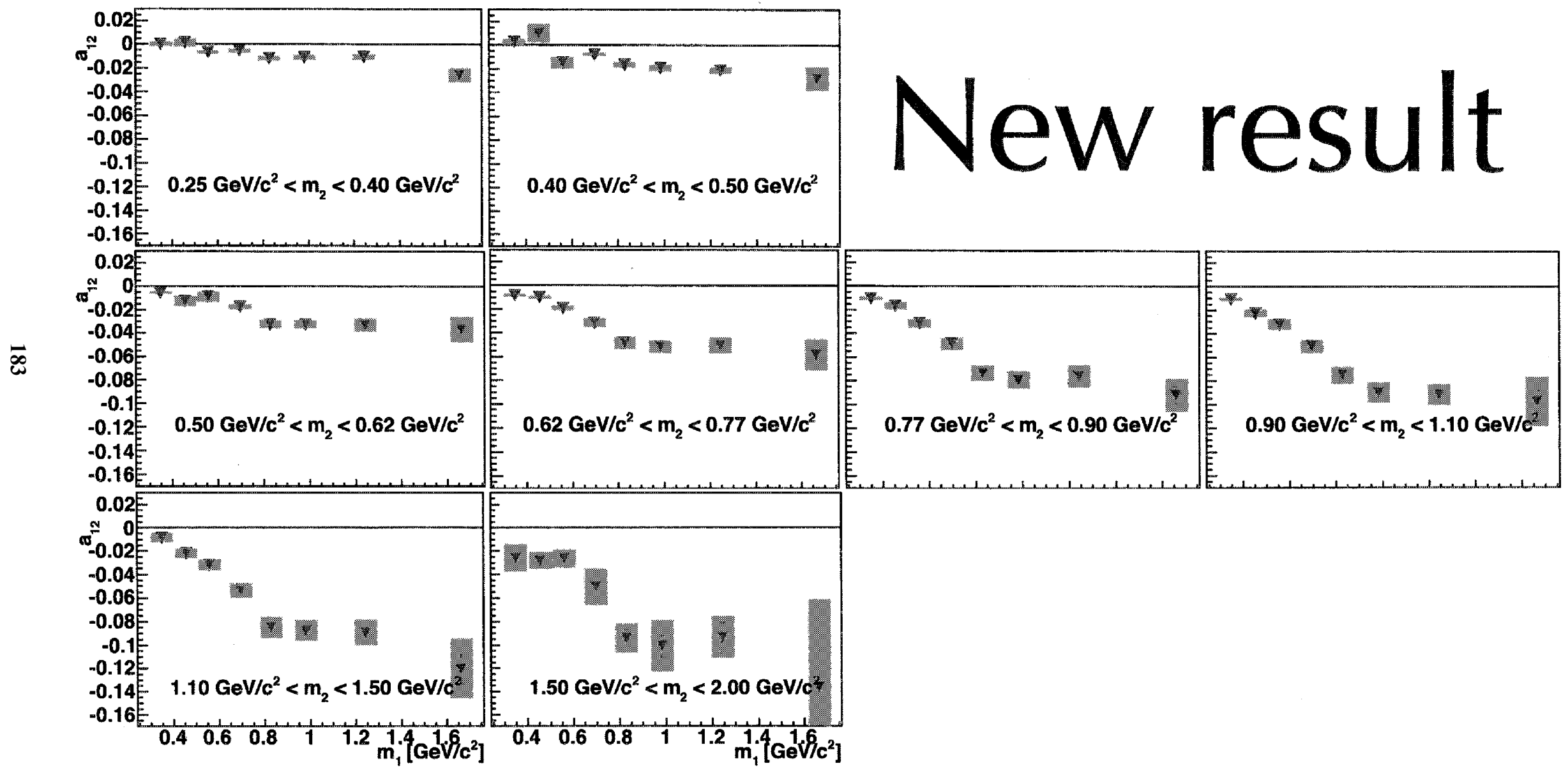

Vossen, Seidl et al. (Belle), arXiv:1104.2425 [hep-ex] 


\section{BELLE data}
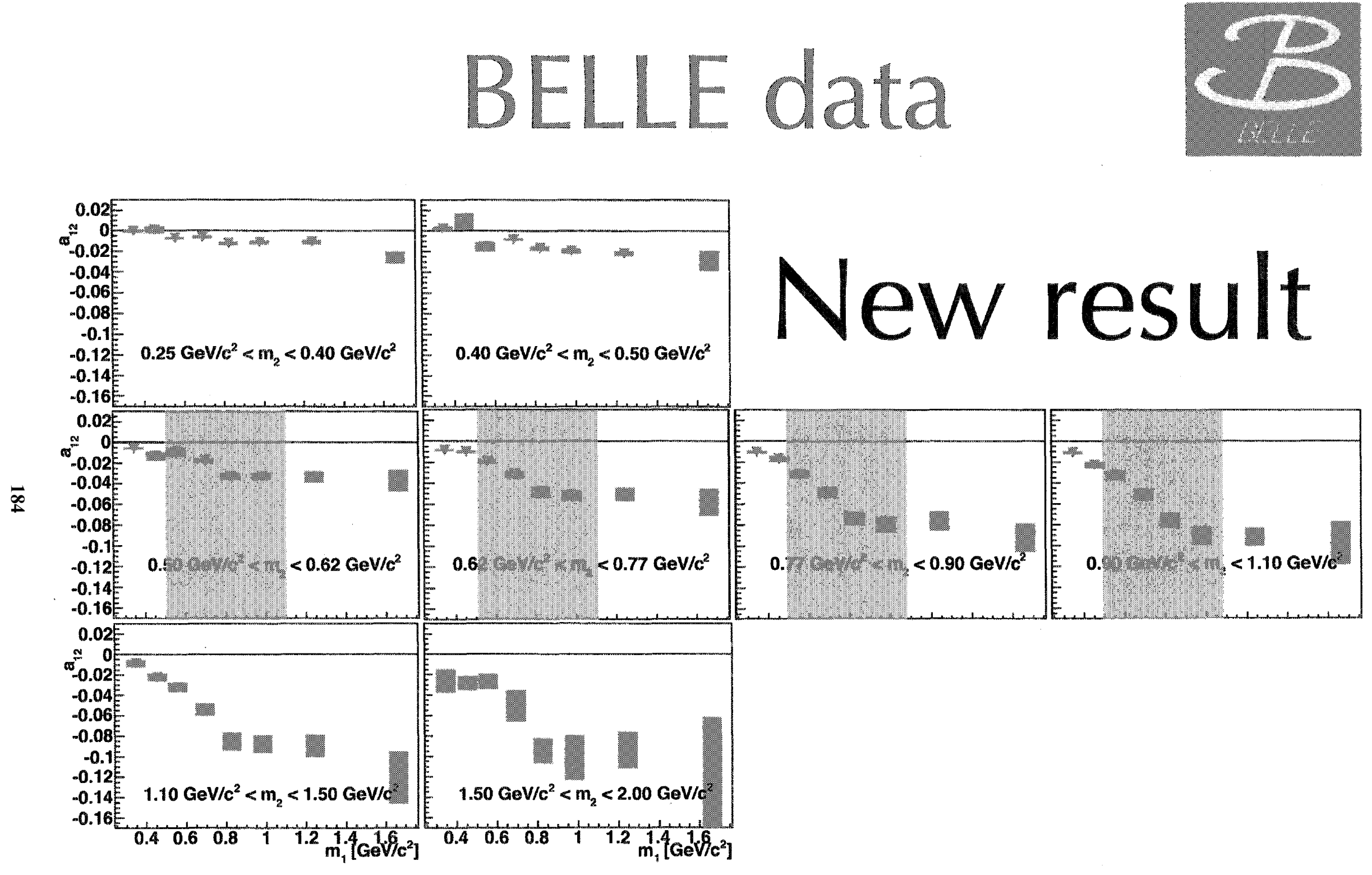

Vossen, Seidl et al. (Belle), arXiv:1104.2425 [hep-ex] 


\section{BELLE data

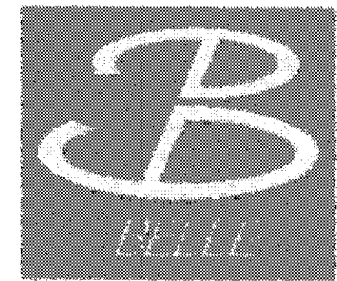

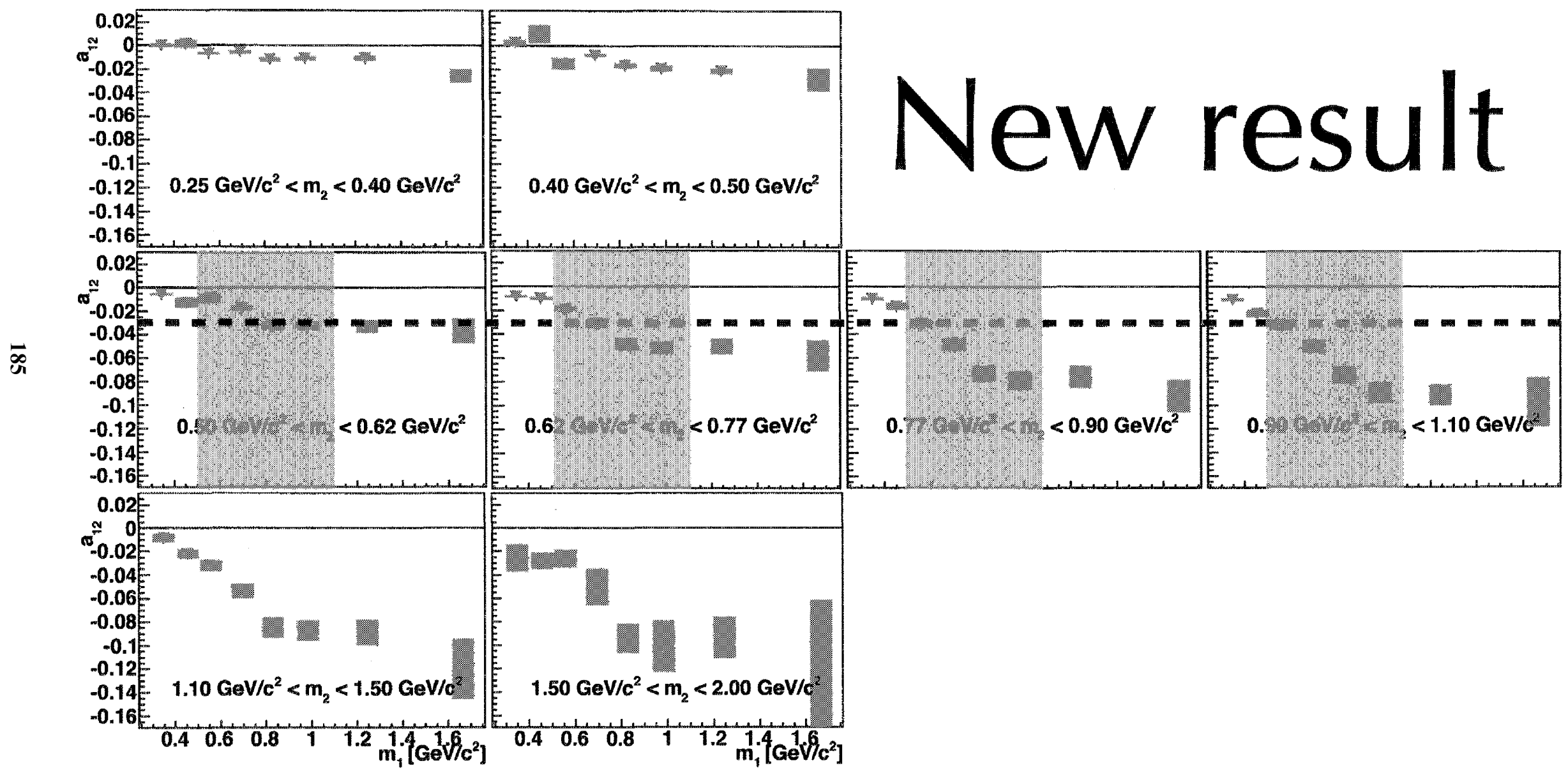

Vossen, Seidl et al. (Belle), arXiv:1104.2425 [hep-ex] 


\section{BELLE data}
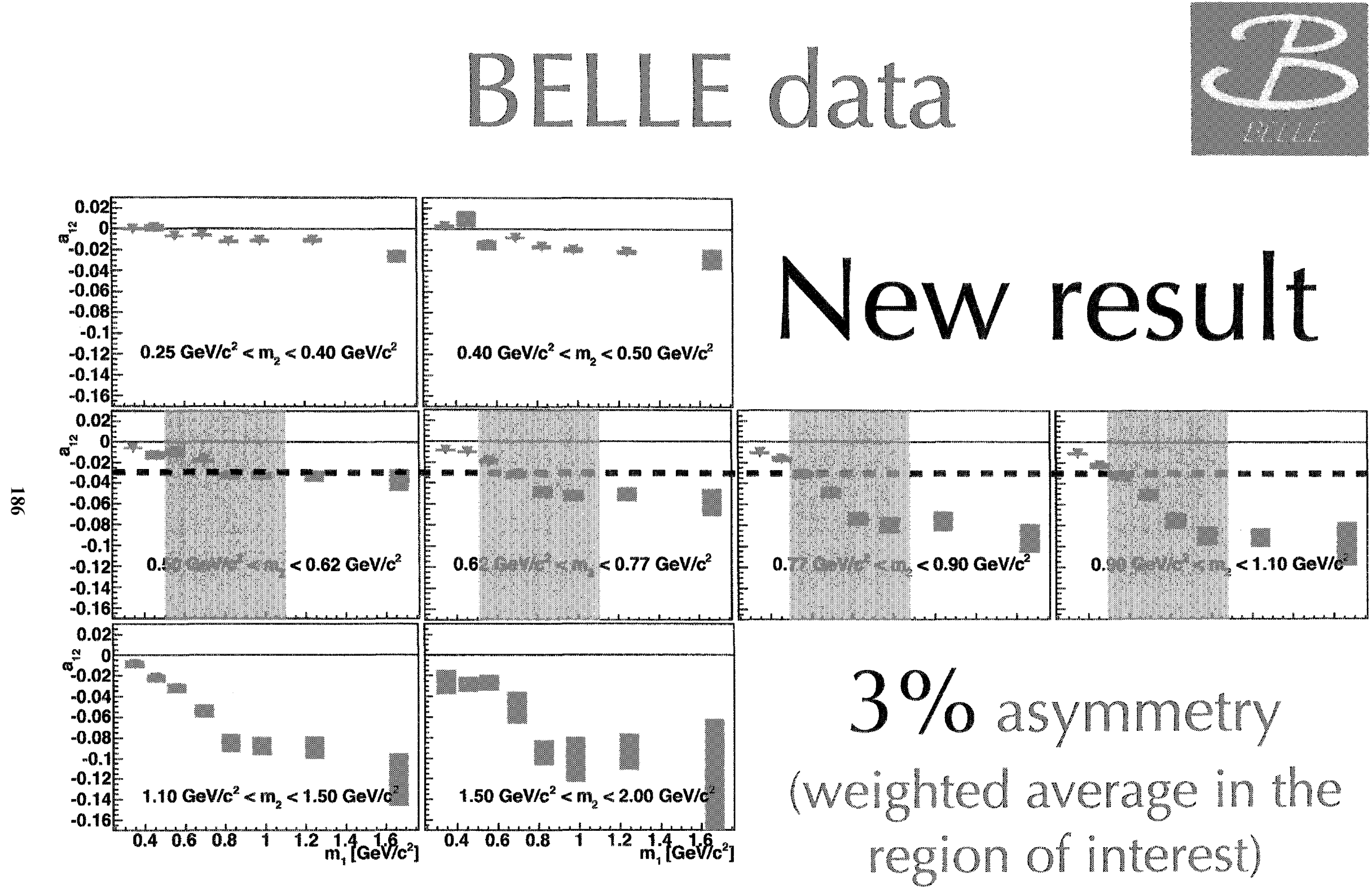

Vossen, Seidl et al. (Belle), arXiv:1104.2425 [hep-ex] 


\section{Simplified expressions}

,

$$
A_{D I S}(x) \approx-\left\langle C_{y}\right\rangle \frac{\left(h_{1}^{u_{v}}(x)-h_{1}^{d_{v}}(x) / 4\right)}{\left(f_{1}^{u+\bar{u}}(x)+f_{1}^{d+\bar{d}}(x) / 4\right)} \frac{n_{u}^{\uparrow}}{n_{u}}
$$

$e^{+} e^{-}$

$$
A_{e+e-} \approx \frac{-\left\langle\sin ^{2} \theta_{2}\right\rangle}{\left\langle 1+\cos ^{2} \theta_{2}\right\rangle} \frac{\langle\sin \theta\rangle\langle\sin \bar{\theta}\rangle 5\left(n_{u}^{\uparrow}\right)^{2}}{5 n_{u}^{2}+n_{s}^{2}+4 n_{c}^{2}}
$$




\section{Simplified expressions}

$\infty$

$$
A_{D I S}(x) \approx-\left\langle C_{y}\right\rangle \frac{\left(h_{1}^{u_{v}}(x)-h_{1}^{d_{v}}(x) / 4\right)}{\left(f_{1}^{u+\bar{u}}(x)+f_{1}^{d+\bar{d}}(x) / 4\right)} \frac{n_{u}^{\dagger}}{n_{u}}
$$

$e^{+} e^{-}$

$$
A_{e+e-} \approx \frac{-\left\langle\sin ^{2} \theta_{2}\right\rangle}{\left\langle 1+\cos ^{2} \theta_{2}\right\rangle} \frac{\langle\sin \theta\rangle\langle\sin \bar{\theta}\rangle 5\left(n_{u}^{\uparrow}\right)^{2}}{5 n_{u}^{2}+n_{s}^{2}+4 n_{c}^{2}}
$$




\section{Simplified expressions}

$$
\begin{aligned}
& A_{D I S}(x) \approx-\left\langle C_{y}\right\rangle \frac{\left(h_{1}^{u_{v}}(x)-h_{1}^{d_{v}}(x) / 4\right)}{\left(f_{1}^{u+\bar{u}}(x)+f_{1}^{d+\bar{d}}(x) / 4\right)} \frac{n_{u}^{\uparrow}}{n_{u}}=\frac{\iint \frac{R}{M_{h}} H_{1, u}^{\varangle}\left(z, M_{h}^{2}\right)}{\iint D_{1, u}\left(z, M_{h}^{2}\right)} \\
& \text { From BELLE: } \frac{n_{u}^{\uparrow}}{n_{u}}=25 \%
\end{aligned}
$$$$
A_{e+e-} \approx \frac{-\left\langle\sin ^{2} \theta_{2}\right\rangle}{\left\langle 1+\cos ^{2} \theta_{2}\right\rangle} \frac{\langle\sin \theta\rangle\langle\sin \bar{\theta}\rangle 5\left(n_{u}^{\uparrow}\right)^{2}}{5 n_{u}^{2}+n_{s}^{2}+4 n_{c}^{2}}
$$ 


\section{Simplified expressions}

$$
\begin{aligned}
& \frac{n_{u}^{\uparrow}}{n_{u}}=\frac{\iint \frac{\boldsymbol{R}}{M_{h}} H_{1, u}^{\varangle}\left(z, M_{h}^{2}\right)}{\iint D_{1, u}\left(z, M_{h}^{2}\right)} \\
& A_{D I S}(x) \approx-\left\langle C_{y}\right\rangle \frac{\left(h_{1}^{u_{v}}(x)-h_{1}^{d_{v}}(x) / 4\right)}{\left(f_{1}^{u+\bar{u}}(x)+f_{1}^{d+\bar{d}}(x) / 4\right)} \frac{n_{u}^{\uparrow}}{n_{u}} \\
& \text { From BELLE: } \frac{n_{u}^{\uparrow}}{n_{u}}=25 \% \\
& A_{e+e-} \approx \frac{-\left\langle\sin ^{2} \theta_{2}\right\rangle}{\left\langle 1+\cos ^{2} \theta_{2}\right\rangle} \frac{\langle\sin \theta\rangle\langle\sin \bar{\theta}\rangle 5\left(n_{u}^{\uparrow}\right)^{2}}{5 n_{u}^{2}+n_{s}^{2}+4 n_{c}^{2}}
\end{aligned}
$$




\section{First glimpses at transversity}

$\varpi$

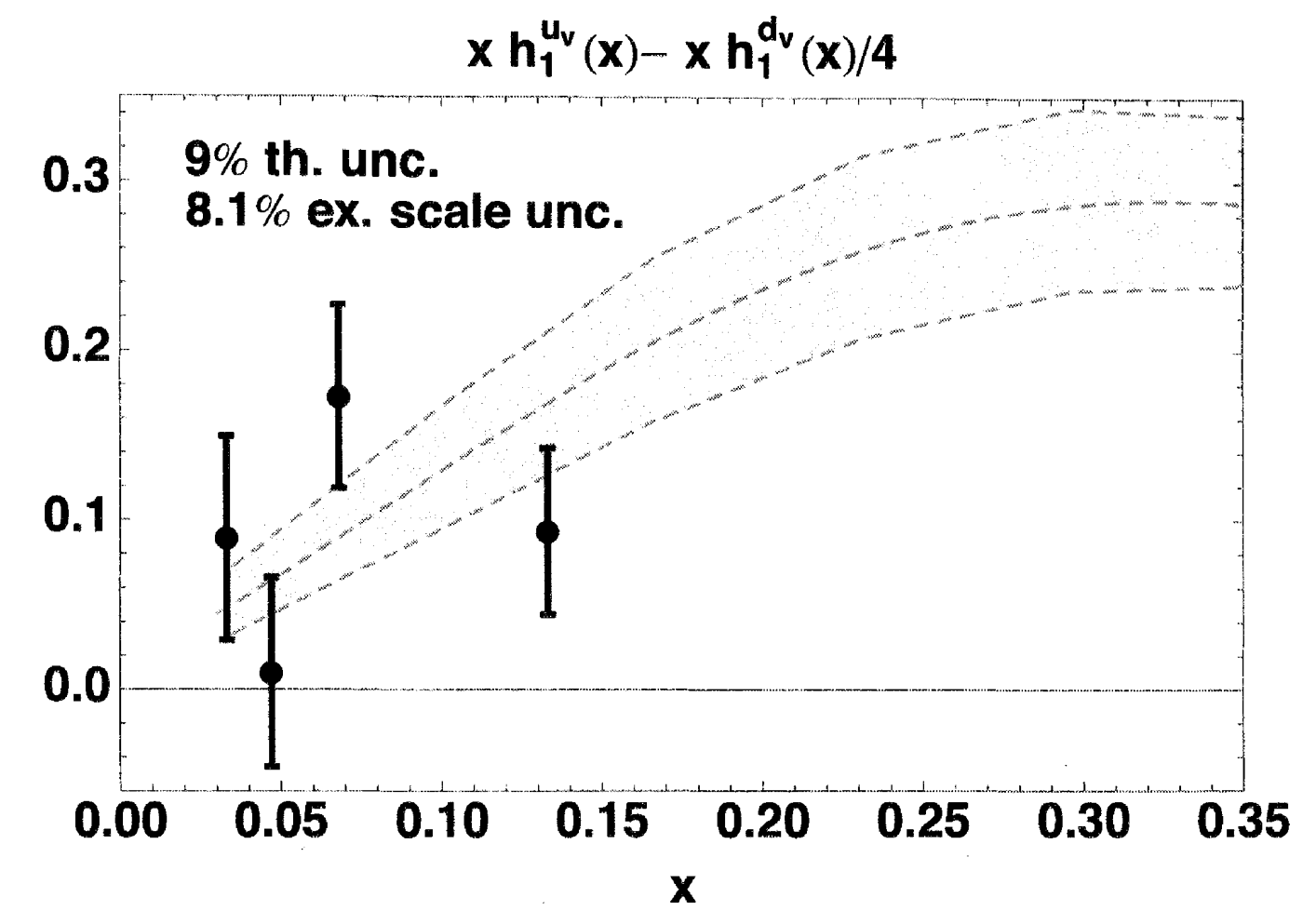

Not in disagreement with Anselmino et al. 


\section{Photon Pair Production}

Marc Schlegel

Institute for Theoretical Physics

University of Tuebingen

in collaboration with Jianwei Qiu \& Werner Vogelsang

arXiv:1103.3861

Summary:

The photon pair production process in proton collision is discussed as a complementary process to the Drell-Yan process in the context of TMDfactorization. It is argued that gluon TMDs can be extracted from photon pair production at RHIC. Estimates are given for the gluonic Boer-Mulders and Sivers effects. 


\section{Photon Pairs from qq-channal}

Parton model tree-level at $\mathrm{O}\left(\alpha_{\mathrm{s}}{ }^{0}\right) \rightarrow$ quark-TMDs!
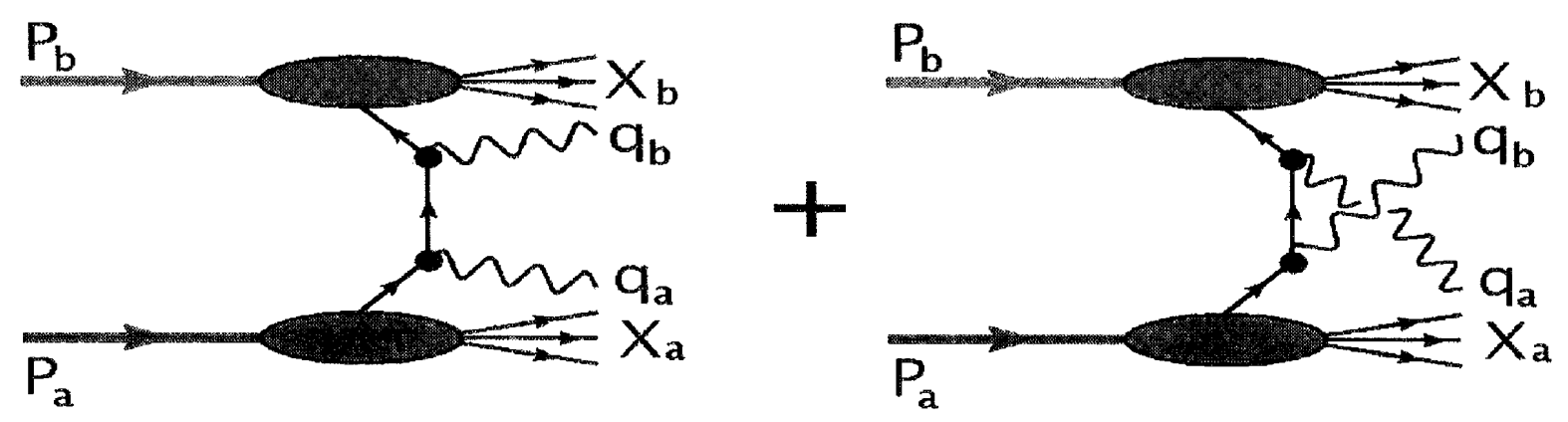

Only relevant at very small $\mathrm{q}_{\mathrm{T}}: \Lambda_{Q C D} \sim q_{T} \ll Q$

$\sqsubseteq \quad\left(\frac{d \sigma}{d^{4} q d \Omega}\right) \propto \int d^{2} k_{a T} \int d^{2} k_{b T} \delta^{(2)}\left(\vec{k}_{a T}+\vec{k}_{b T}-\vec{q}_{T}\right) \operatorname{Tr}\left[\Phi\left(x_{a}, \vec{k}_{a T}\right) H\left(x_{a}, x_{b}, q_{a}, q_{b}\right) \Phi\left(x_{b}, \vec{k}_{b T}\right) H^{\dagger}\right]+\mathcal{O}\left(\frac{M}{Q}\right)$

$k_{T}$ - correlator: $\Phi_{i j}\left(x, \vec{k}_{T}\right)=\left.\int \frac{d z^{-} d^{2} z_{T}}{(2 \pi)^{2}} \mathrm{e}^{i k \cdot z}\left\langle P, S\left|\bar{q}_{j}(0) \mathcal{W}^{? / D Y}[0 ; z] q_{j}(z)\right| P, S\right\rangle\right|_{z^{+}=0}$

Main result of the TMD tree-level formalism:

$\left(\frac{d^{6} \sigma^{h h \rightarrow \gamma \gamma X}}{d y d Q^{2} d^{2} q_{T} d \Omega}\right)\left(\Lambda \sim q_{T} \ll Q\right)=\frac{2}{\sin ^{2} \theta}\left(\frac{d \sigma^{h h \rightarrow l^{+} l^{-} X}}{d y d Q^{2} d^{2} q_{T} d \Omega}\right)\left(\Lambda \sim q_{T} \ll Q \mid e_{q} \rightarrow e_{q}^{2}\right)$ 


\section{Gluon TMDs in photon pair production}

Gluon TMDs in pp-collisions with colored final state:

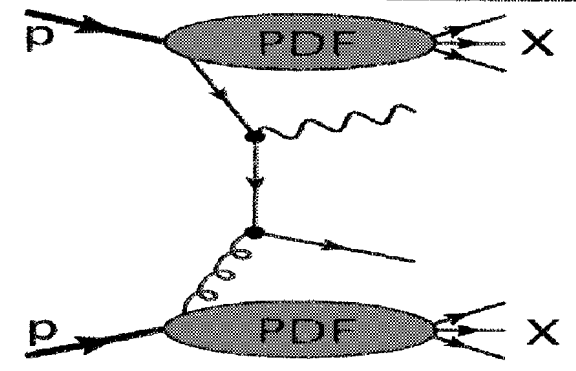

TMD-Factorization (?)
Gluon TMDs in Heavy-quark production in ep-collisions:

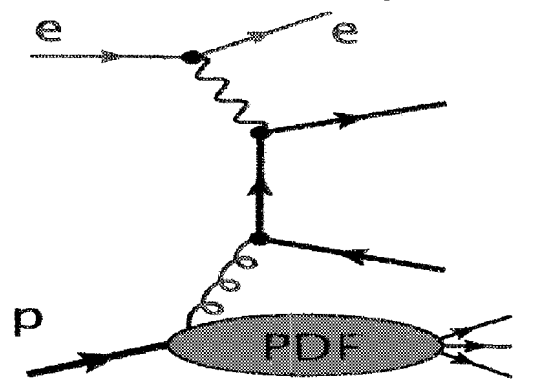

Wait for EIC

Feature of photon pair production $\rightarrow$ direct sensitivity to gluon TMDs at $\mathrm{O}\left({ }_{\mathrm{s}}^{2}\right)$
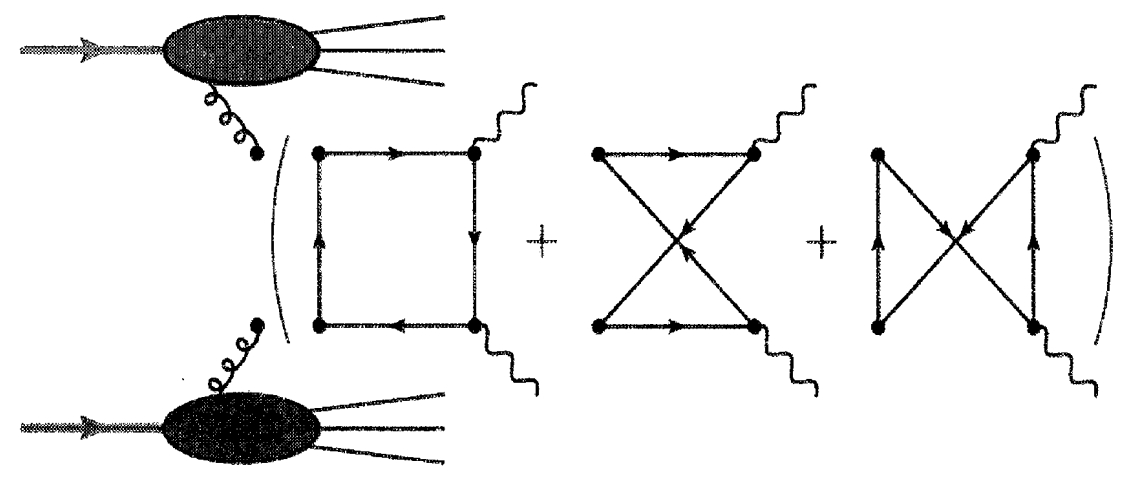

- No colored final state

- Box diagrams finite

- Potentially large gluon distributions

- New Observables, e.g. $\operatorname{Cos}(4 \phi)$ 


\section{Unpolanized Cross Section}

Six structures for the unpolarized cross section $\left(q_{I} \ll Q\right)$.

$$
\begin{aligned}
\frac{\mathrm{d} \sigma_{U U}}{\mathrm{~d}^{4} q \mathrm{~d} \Omega} \sim & \left(\frac{2}{\sin ^{2} \theta}\right)\left(\left(1+\cos ^{2} \theta\right)\left[f_{1}^{q} \otimes f_{1}^{\bar{q}}\right]+\cos (2 \phi) \sin (2 \theta)\left[h_{1}^{\perp q} \otimes h_{1}^{\perp \bar{q}}\right]\right) \\
& +\left(\frac{\alpha_{s}}{2 \pi}\right)^{2}\left(\mathcal{F}_{1}\left[f_{1}^{g} \otimes f_{1}^{g}\right]+\mathcal{F}_{2}\left[h_{1}^{\perp g} \otimes h_{1}^{\perp g}\right]+\cos (2 \phi) \mathcal{F}_{3}\left[h_{1}^{\perp g} \otimes f_{1}^{g}+f_{1}^{g} \otimes h_{1}^{\perp g}\right]+\cos (4 \phi) \mathcal{F}_{4}\left[h_{1}^{\perp g} \otimes h_{1}^{\perp g}\right]\right)
\end{aligned}
$$

$\mathcal{F}_{i}$ : non-trivial functions of $\sin (\theta)$ and $\cos (\theta)$ (Logarithms)

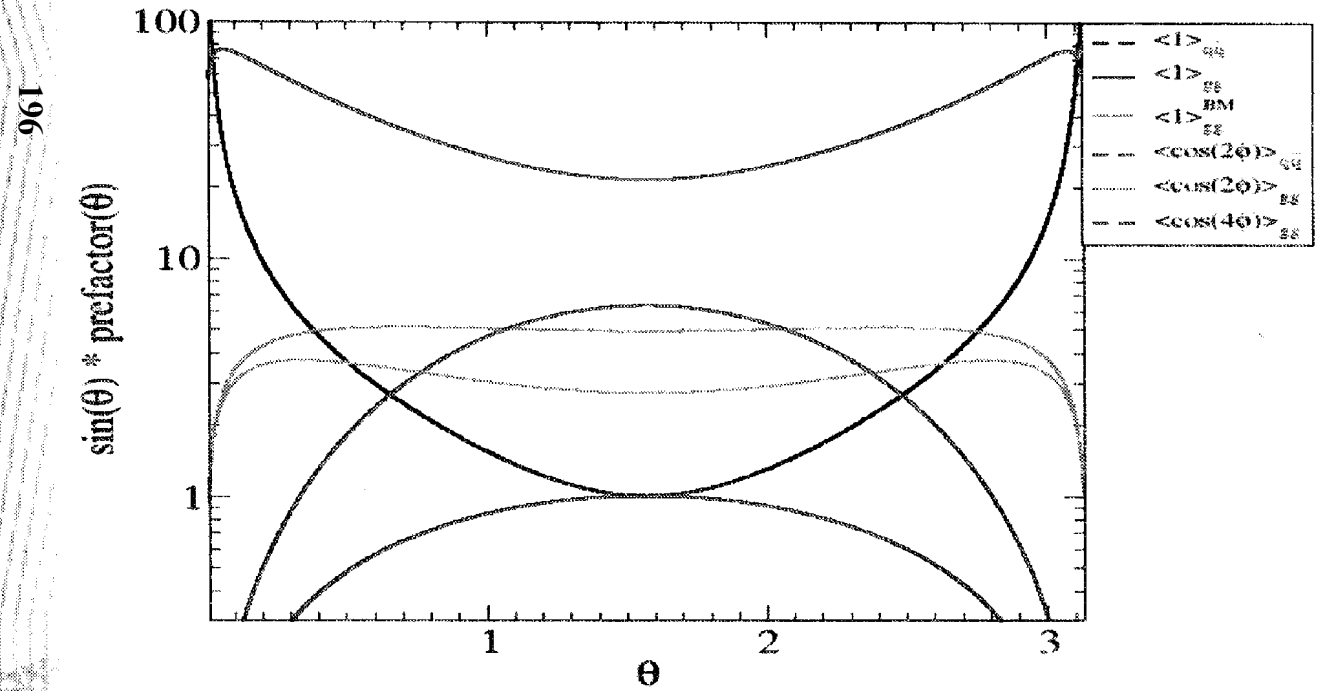

LHC: Diphotons $\rightarrow$ main channel for Higgs-Prod.

$\rightarrow$ Background process: diphotons via quark-box

$\rightarrow$ gluon TMD (unpol., BM) feasible
- q9 singular for $\theta \rightarrow 0, \pi$

$\rightarrow P_{\text {T }}($ or $\theta)$-cuts for each photon

- $\cos (4 \phi)$ induced by gluon BM- functions,

$\rightarrow$ no corresponding quark / DY term.

- powerful in combination with DY

$\rightarrow$ even gluon TMD $f_{1}$ unknown.

- $\cos (2 \phi)$ determines sign of gluon BM-function.

- Same angular structure found in collinear resummation formulas for higher qT.

(Balazs et al., Catani \& Grazzini)

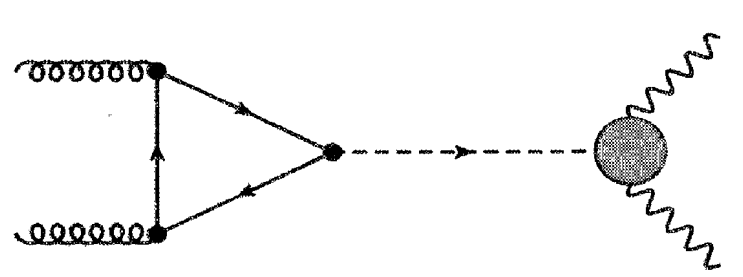




\section{(Maximal) Gluonic BM-effect at RHIC}

Estimates at RHIC $\left(S^{1 / 2}=500 \mathrm{GeV}\right) \rightarrow$ Gluon (and Quark!) TMDs unknown at RHIC energy

Saturation of Positivity bounds:

$\left|h_{1}^{\perp, g}\right| \leq \frac{2 M^{2}}{k_{T}^{2}} f_{1}^{g}\left|h_{1}^{\perp}, q\right| \leq \frac{M}{k_{T}} f_{1}^{q}$

Gaussian Ansatz:

$f_{1}^{q / g}\left(x, k_{T}^{2}\right)=f_{1}^{q / g}(x) \mathrm{e}^{-k_{T}^{2} /\left\langle k_{T, q / g}^{2}\right\rangle}$

Further assumption:

$\left\langle k_{T, q}^{2}\right\rangle=\left\langle k_{T, g}^{2}\right\rangle=0.5 \mathrm{GeV}^{2}$

$P_{I}$-cut for photons:

$p_{T}^{\gamma}>1 \mathrm{GeV}$

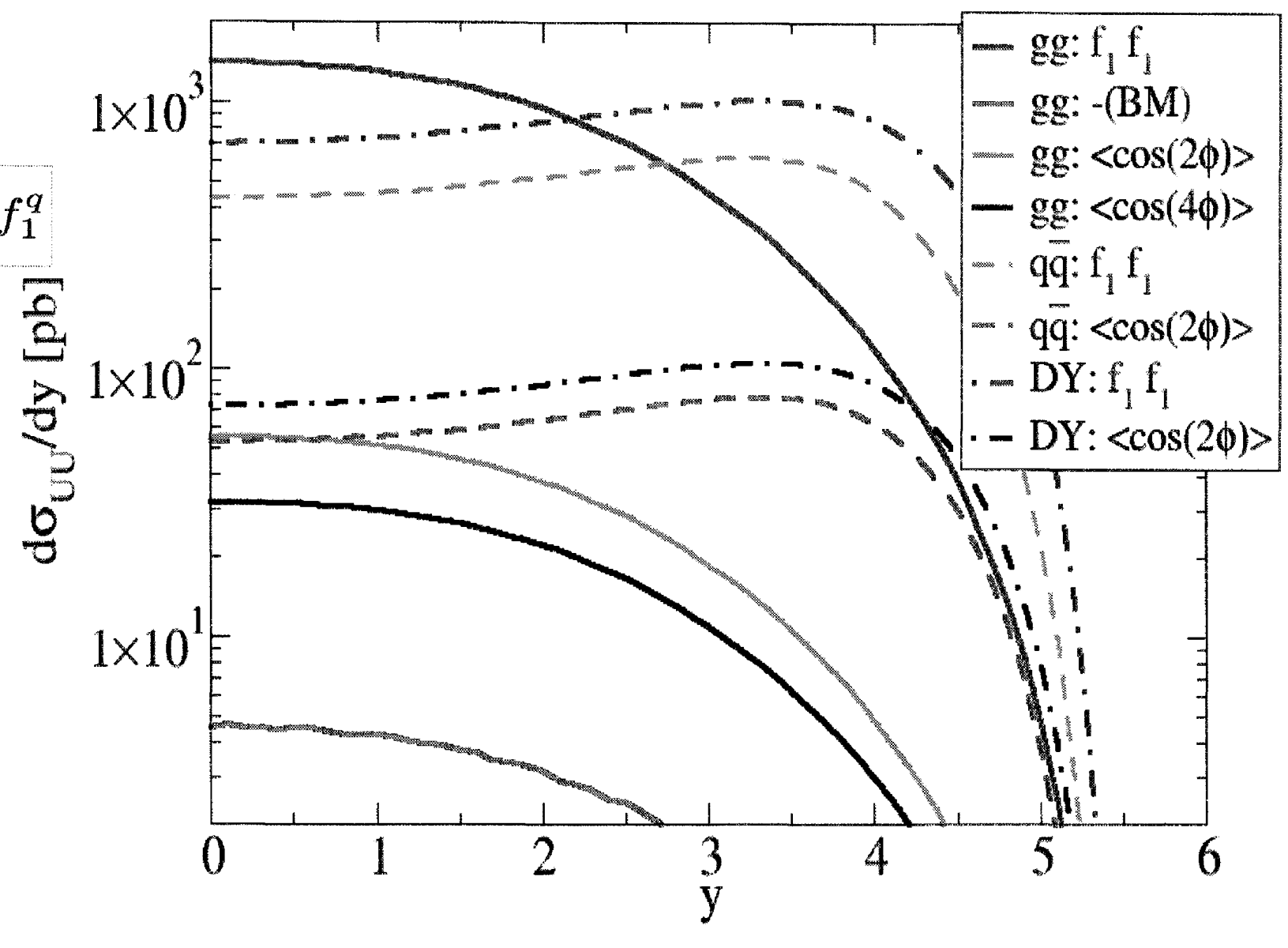

- Gluons at midrapidity, quarks at large rapidity

- BM-contribution to $\phi$-indep. CS small

- $\langle\cos (4 \phi)>\leq 1 \%$ (depend. on saturation) 


\section{Gluonic Sivers-effect at RHIC}

Four structures for the $\phi$-indep. transv. Single-Spin Asymmetry

$\begin{aligned} & \frac{\mathrm{d} \sigma_{\mathrm{TU}}}{\mathrm{d}^{4} q \mathrm{~d} \Omega} \sim S_{T} \sin \phi_{S}\left[\frac{2}{\sin ^{2} \theta}\left(1+\cos ^{2} \theta\right)\left[f_{1 T}^{\perp, q} \otimes f_{1}^{\bar{q}}\right]\right. \\ &\left.+\left(\frac{\alpha_{s}}{2 \pi}\right)^{2}\left(\mathcal{F}_{1}\left[f_{1 T}^{\perp, g} \otimes f_{1}^{g}\right]+\mathcal{F}_{2}\left[h_{1}^{g} \otimes h_{1}^{\perp, g}\right]+\mathcal{F}_{2}\left[h_{1 T}^{\perp, g} \otimes h_{1}^{\perp, g}\right]\right)\right]+\ldots\end{aligned}$

$\ddot{\circ}$

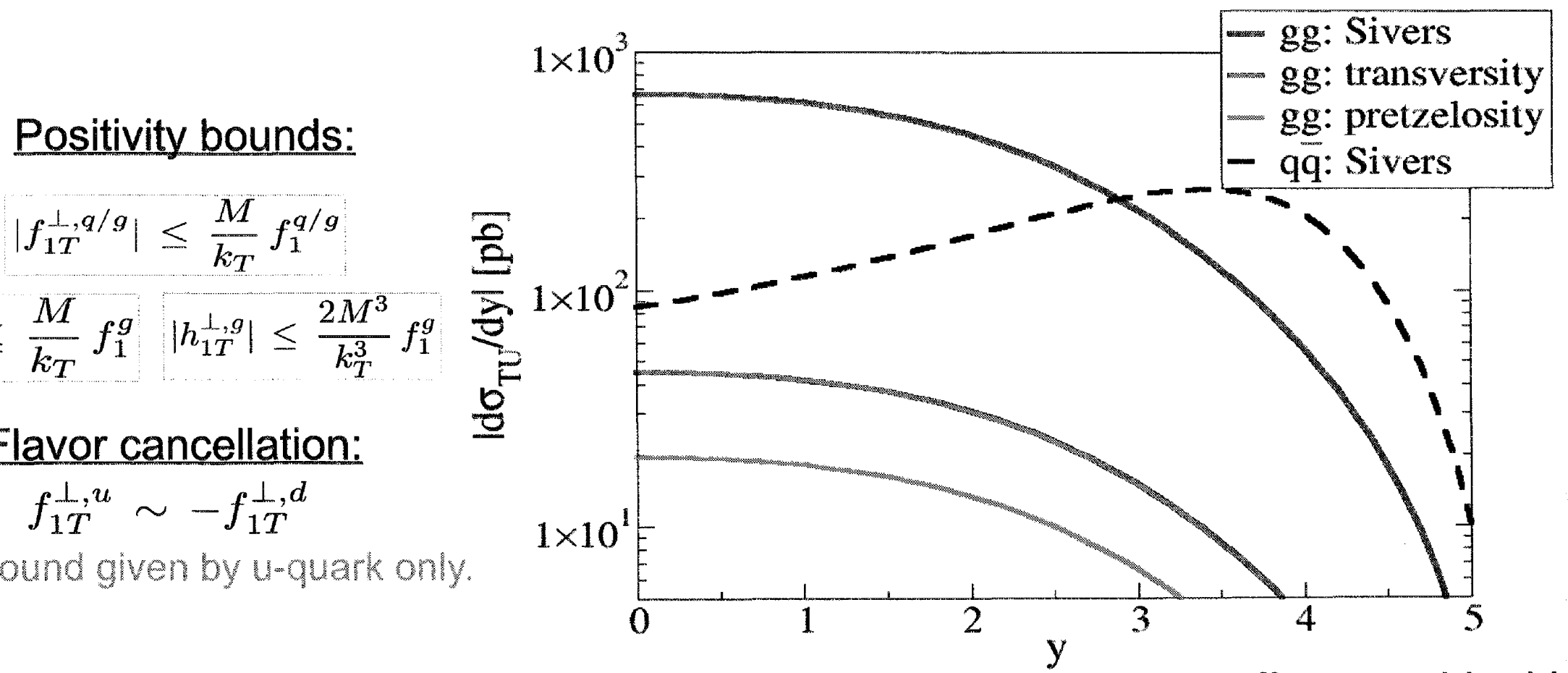

- Sign of Sivers function not predicted by positivity $\rightarrow$ quark and gluon effects could add.

- Gluonic effects at midrapidity, quark effects at large rapidities.

- Effects by gluon transversity / pretzelosity small. 


\title{
Many body QCD: from RHIC ( \& LHC) to the EIC
}

\author{
Raju Venugopalan
}

Brookhaven National Laboratory

క్

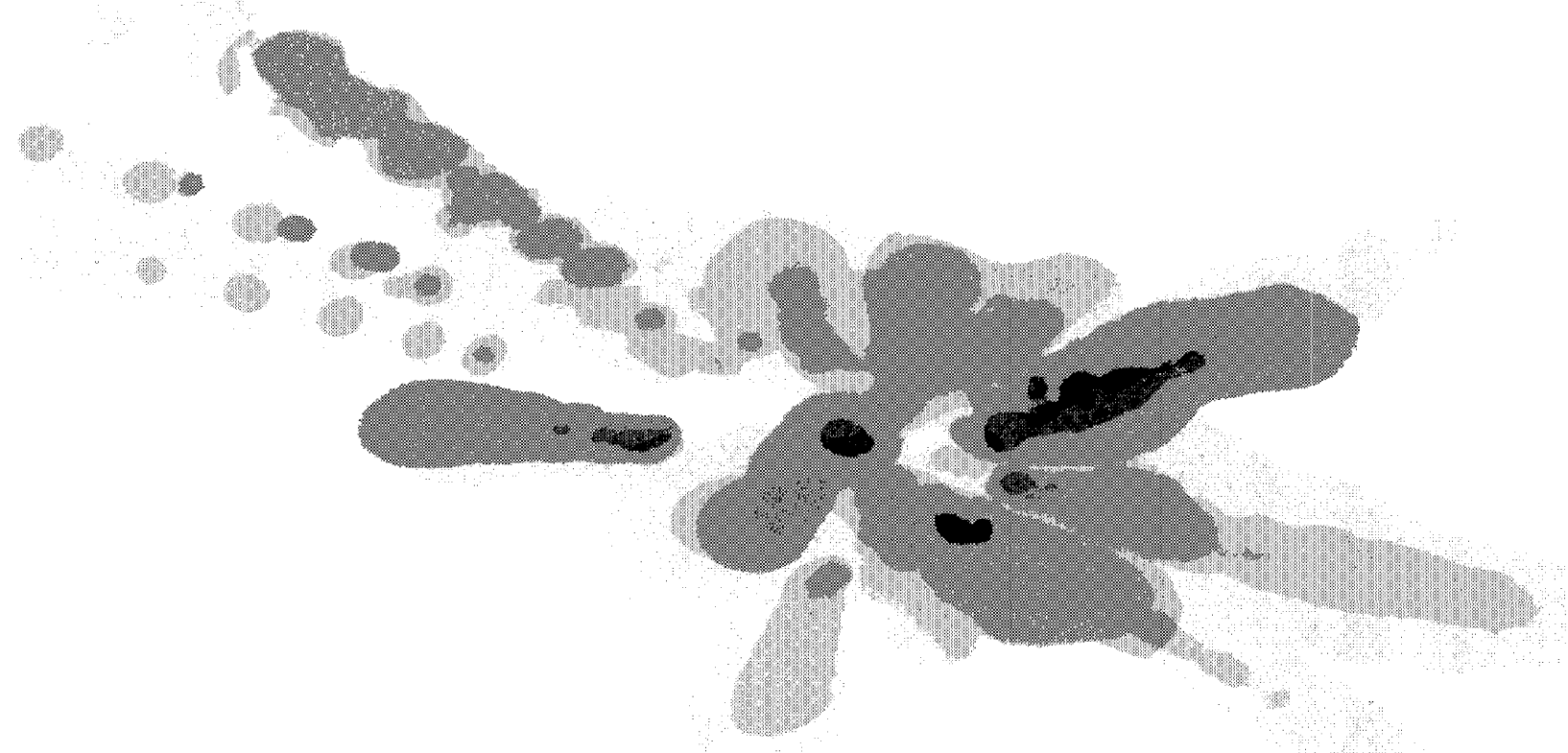

BNL Drell-Yan workshop, May 11-13, 2011 


\section{Semi-inclusive DIS: quadrupole evolution}
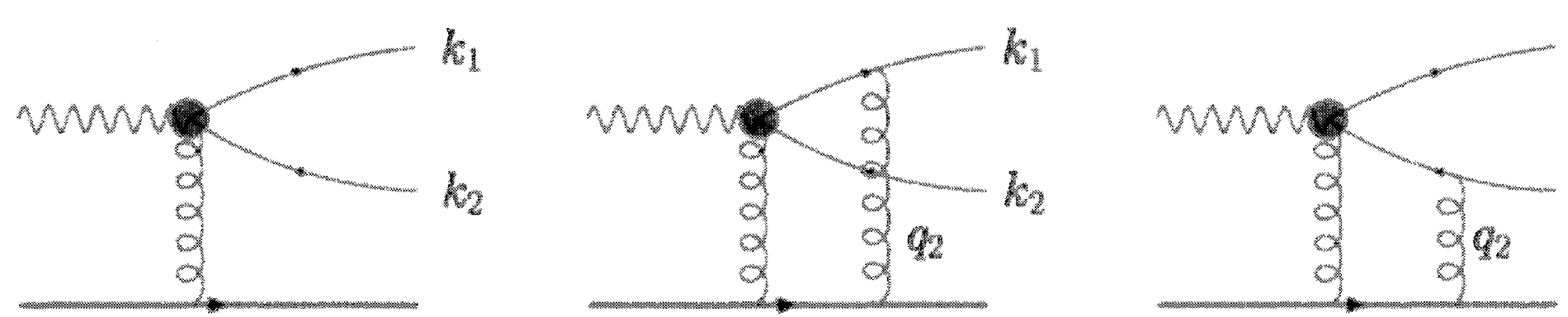

๕ั

$$
\begin{aligned}
& \frac{d \sigma^{\gamma_{\mathrm{T}, \mathrm{L}}^{*} A \rightarrow q \bar{q} X}}{d^{3} k_{1} d^{3} k_{2}} \propto \int_{x, y, \bar{x} \bar{y}} e^{i k_{1 \perp} \cdot(x-\bar{x})} \\
& D(x, y)=\frac{1}{N_{C}}\left\langle\operatorname{TY}\left(V_{x} V_{y}^{\dagger}\right)\right\rangle_{Y}
\end{aligned}
$$$$
Q(x, y ; \bar{y}, \bar{x})=\frac{1}{N_{c}}\left\langle\operatorname{Tr}\left(V_{x} V_{\bar{x}} V_{y} V_{y}\right)\right\rangle_{Y}
$$

Dominguez, Marquet, Xiao,Yuan (2011)

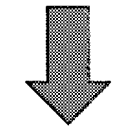

Cannot be further simplified a priori even in the large $\mathrm{N}_{c}$ limit 


\section{Universality: Di-jets in $\mathrm{p} / \mathrm{d}-\mathrm{A}$ collisions}

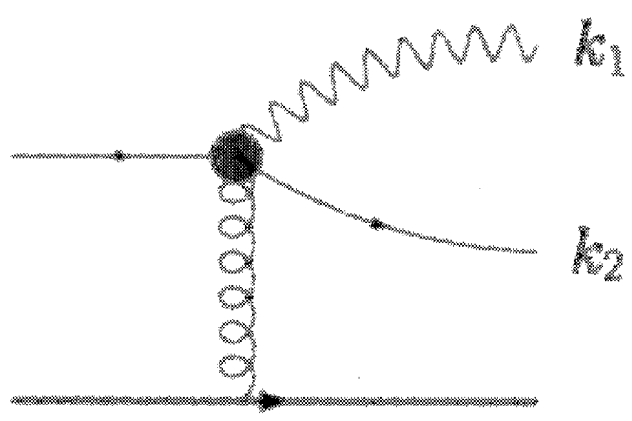

$\tilde{\Theta}$
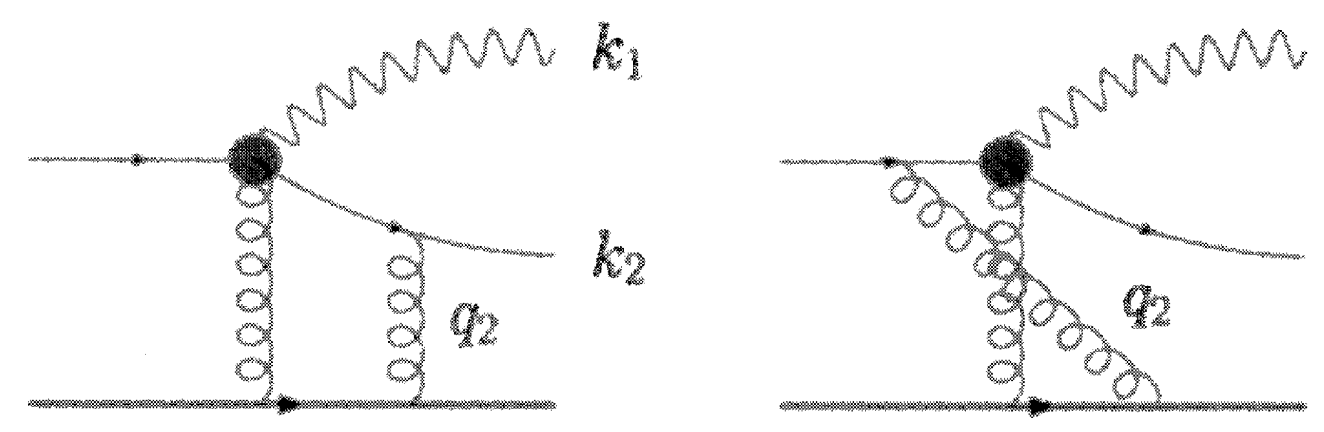

Jalilian-Marian, Kovchegov (2004) Marquet (2007)

Dominguez,Marquet, Xiao, Yuan (2011)

$$
\begin{aligned}
& \frac{d \sigma^{q A-q g X}}{d^{3} k_{1} d^{3} k_{2}} \propto \int_{x, y, x, y} e^{i k_{11} \cdot(x-\bar{x})} e^{i k_{2} \perp \cdot(y-y)}\left[S_{6}(x, y, \bar{x}, \bar{y})-S_{4}(x, y, v)-\ldots\right] \\
& \frac{N_{c}}{2 C_{F}}\left\langle Q(x, y \bar{y}, \bar{x}) D(y, \bar{y})-\frac{D(x, \bar{x})}{N_{c}}\right\rangle \quad \frac{N_{c}}{2 C_{F}}\left\langle D(x, y) D(\bar{y}, \bar{x})-\frac{D(x, \bar{x})}{N_{c}}\right\rangle
\end{aligned}
$$

Fundamental ingredients are the universal dipoles and quadrupoles 


\section{B-JIMWLK hierarchy: Langevin realization}

Numerical evaluation of Wilson line correlators on 2+1-D lattices:

$$
\langle\mathcal{O}[U]\rangle_{Y}=\int D[U] W_{Y}[U] \mathcal{O}[U] \longrightarrow \frac{1}{N} \sum_{U \in W} \mathcal{O}[U]
$$

Langevin eqn:

\section{Gaussian random variable}

$\widetilde{\sim}$

$$
\begin{gathered}
\partial_{Y}\left[V_{x}\right]_{i j}=\left[V_{x} i t^{a}\right]_{i j}\left[\int d^{2} y\left[\mathcal{E}_{x y}^{a b}\right]_{k}\left[\xi_{y}^{b}\right]_{k}+\sigma_{x}^{a}\right] \\
\mathcal{E}_{x y}^{a b}=\left(\frac{\alpha_{S}}{\pi^{2}}\right)^{1 / 2} \frac{(x-y)_{k}}{(x-y)^{2}}\left[1-U_{x}^{\dagger} U_{y}\right]^{a b} \quad \sigma_{x}^{a}=-i\left(\frac{\alpha_{S}}{2 \pi^{2}} \int d^{2} z \frac{1}{(x-z)^{2}} \operatorname{Tr}\left(T^{a} U_{x}^{\dagger} U_{z}\right)\right) \\
\text { "square root" of JIMWLK kernel }
\end{gathered}
$$

$\square$ Initial conditions for V's from the MV model

$\square$ Daughter dipole prescription for running coupling 


\section{Numerical results-IV}

Lappi,Schenke, RV

How about the quantity $S_{6}$ containing quadrupoles that appear in di-hadron correlations?
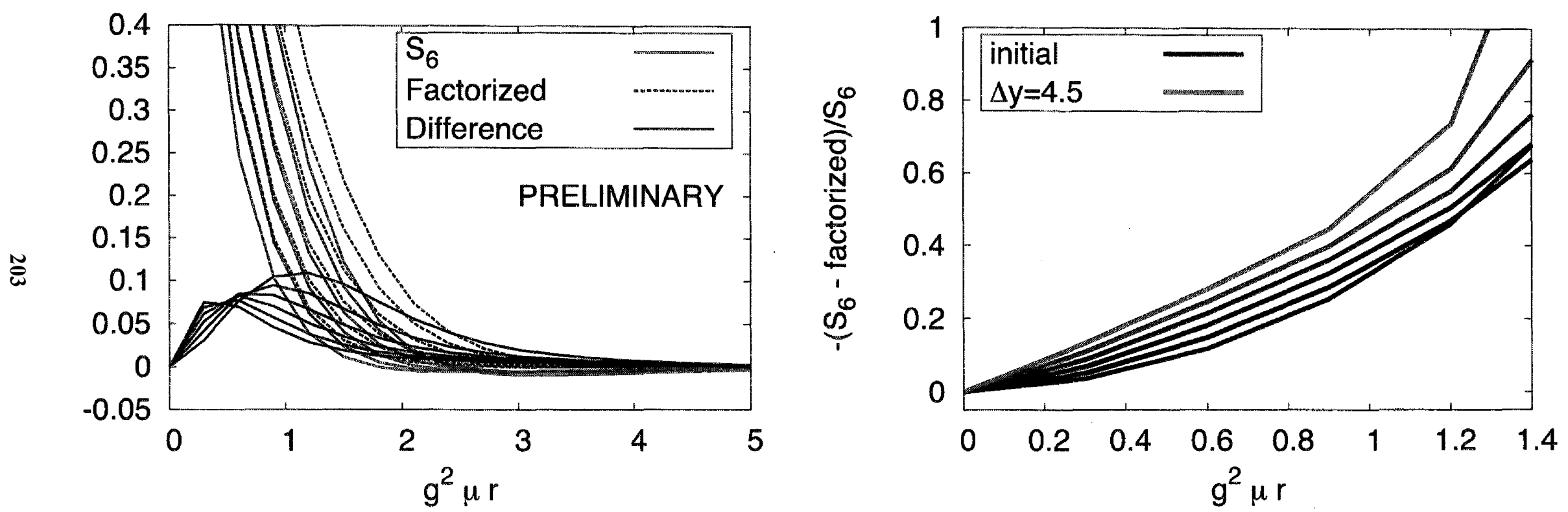

Violations large for large $r$ and for large $Y$ (i.e., when saturation effects are important) - confirming analytical estimates 


\section{Outlook - I}

The JIMWLK hierarchy contains non-trivial "many body" correlations -these are now being explored using numerical and analytical techniques

- It is likely that they could be inferred (given sufficient precision) from experiments thereby providing key insight into QCD many body dynamics in the Regge-Gribov limit

- There are many open questions that hopefully will be resolved in the next decade, such as i) NLL corrections, ii) matching to OPE based analyses at larger $x$ and $Q^{2}$ 


\title{
Transverse Single Spin Asymmetries for Drell-Yan production
}

\author{
John Collins \\ Penn State University
}

\begin{abstract}
I review the arguments for the importance of measuring transverse single spin asymmetries for the Drell-Yan process. On the theory side, key elements of factorization are Wilson lines in the definition of parton densities; these correspond to partonic color flows relative to a hard scattering. The appropriate choice of the Wilson line directions manifests itself in a testable way in the predicted change in sign of Sivers function between SIDIS and DY.

If experiments find that this prediction fails, and this finding survives close scrutiny, we would have to reconsider our understanding of QCD hard scattering.
\end{abstract}




\section{Drell-Yan has different pdfs}
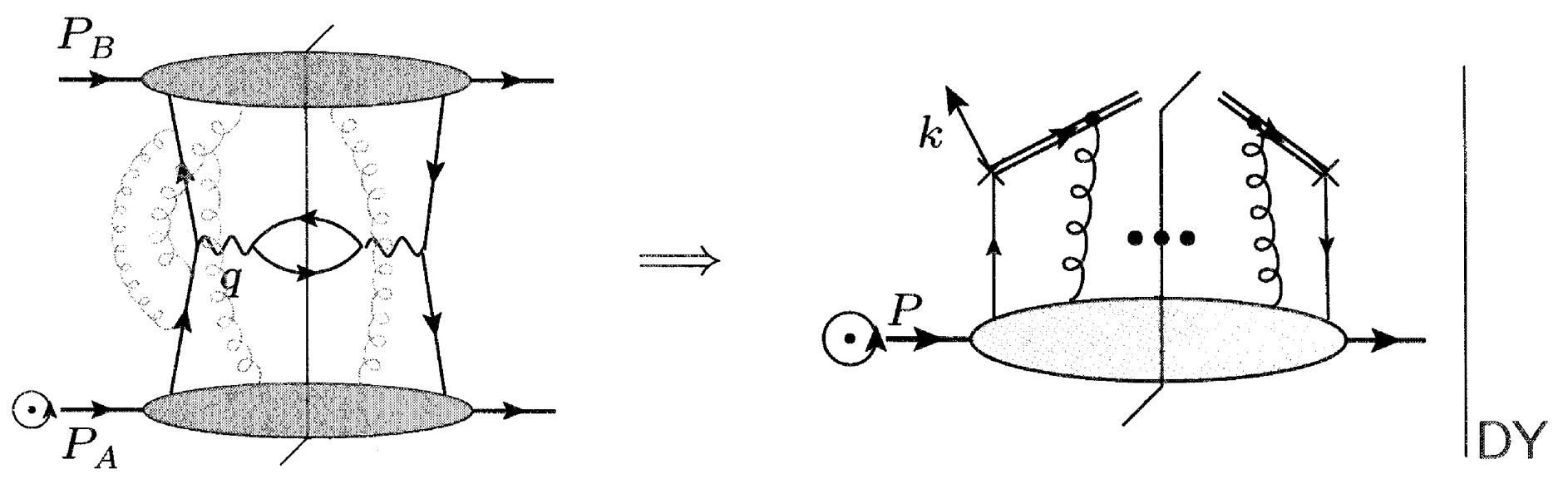

ڤั

with past-pointing WLs:
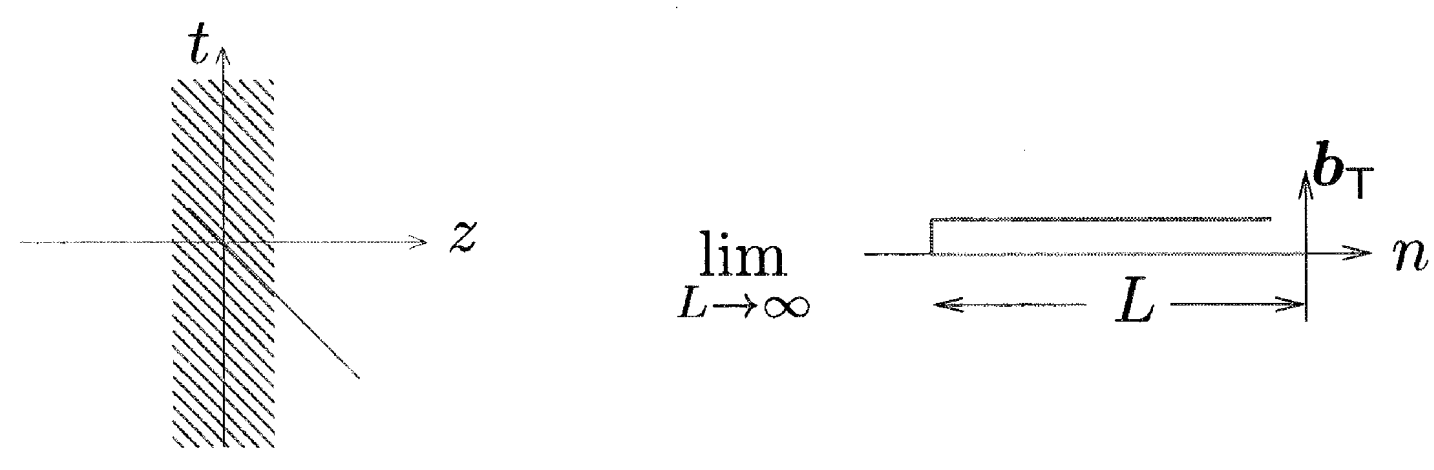

(Need cancellation of f.s.i.; inclusive Drell-Yan only.)

(Proof of TMD factorization - JCC's "Foundations of perturbative QCD".) 


\section{Experimentally accessible consequence of WL: Sign change of} Sivers function

Relate pdfs in SIDIS and DY by $T P$ transformation

Changes:

- Wilson lines:

Future-pointing for SIDIS pdfs $\stackrel{T P}{\Longleftrightarrow}$ past-pointing for DY pdfs

- States:

$$
\left.\left|\mathbf{P}, \boldsymbol{s}_{\mathrm{T}}\right\rangle \Longleftrightarrow|\stackrel{P}{\Longleftrightarrow}|-\mathbf{P}, \boldsymbol{s}_{\mathrm{T}}\right\rangle \Longleftrightarrow\left|\mathrm{P},-\boldsymbol{s}_{\mathrm{T}}\right\rangle
$$

- Hence for pdfs:

- normal pdf ${ }_{D Y}=$ normal $\mathrm{pdf}_{\mathrm{DIS}}$

- But Sivers $\mathrm{DY}=-$ Sivers $_{\mathrm{DIS}}$ 


\section{Prediction for DY}

To have a prediction, $x_{\text {in polarized proton }}$ must be in Hermes region

\section{SIDIS}

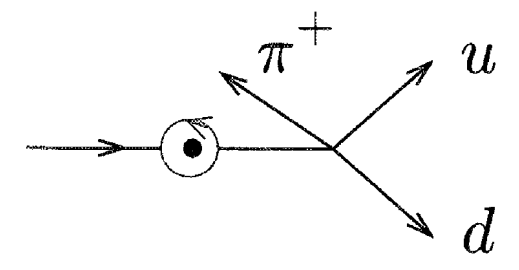

敛
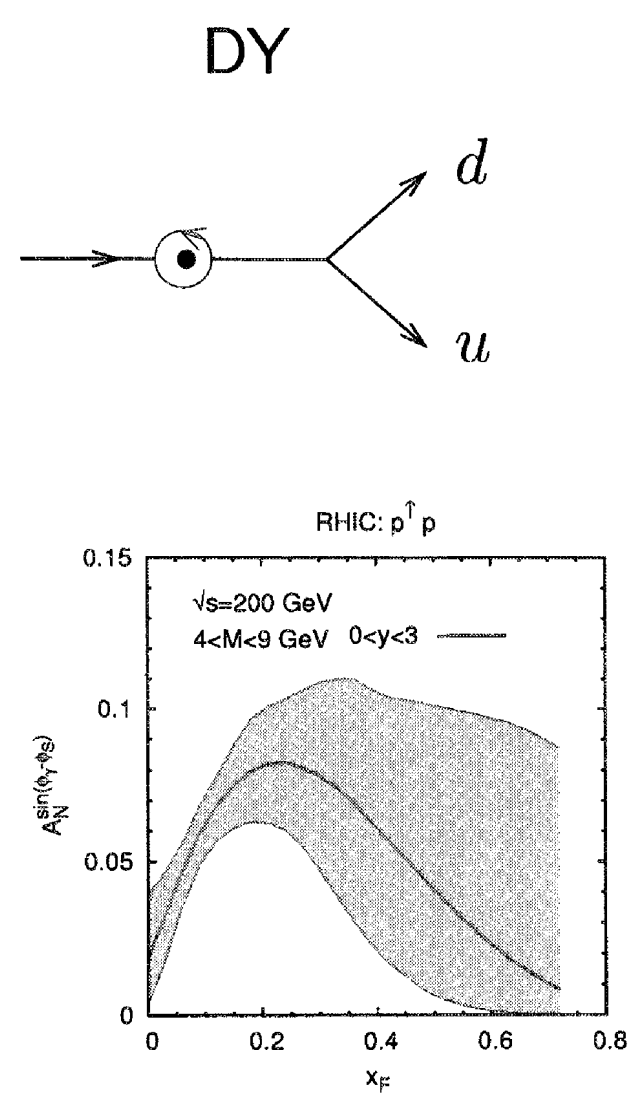

Prediction w/o (CSS) evolution for TMDs

(Anselmino et al. Phys.Rev. D79 (2009) 054010)

For $\mathrm{d} \sigma / \mathrm{d} q \mathrm{~d} \Omega$ : distribution $\propto \sin \left(\phi_{q}-\phi_{s}\right)\left(1+\cos ^{2} \theta\right)+$ Boer-Mulders term 


\section{Reliability of theoretical framework}

QCD

Kinematic approximations

Disentangle gluons into Wilson lines

Cancellation of spectator interactions

factorization

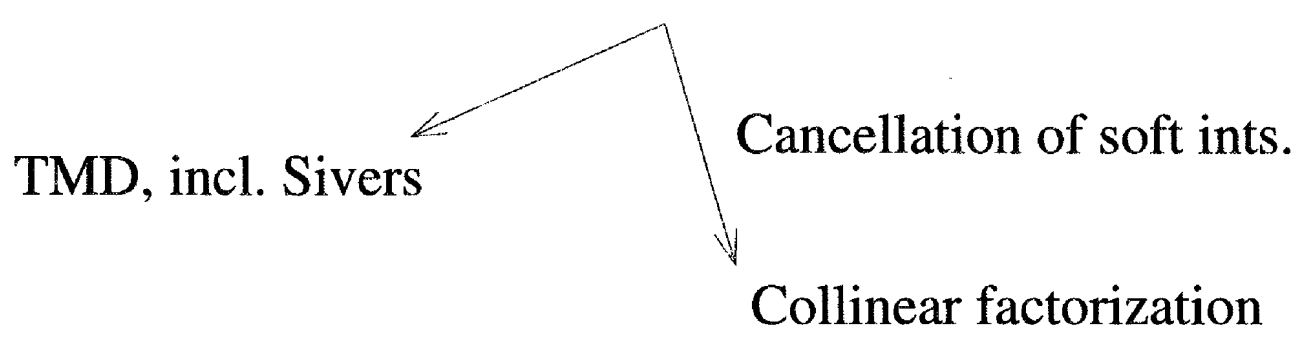

Any problems with Sivers function impact issues critical to all kinds of factorization.

(Unpolarized) factorization survived many tests, so probability of failure is low.

Wilson lines encode space-time locations of color flow relative to hard scattering. 


\section{Conclusions}

- Sivers function gives stress test of our understanding of QCD parton dynamics in hard scattering, especially of space-time locations of color flows

(Key issues were hidden until recently!)

- SSA in Drell-Yan is clean test case with predictions deduced from SIDIS data and unpolarized Drell-Yan

But remember CSS evolution (or equivalent) in making predictions.

- Disquieting data for $p^{\uparrow} p \rightarrow \pi X$

- But than $p^{\uparrow} p \rightarrow \pi X$ is harder for theory than Drell-Yan 
Opportunities for Drell-Yan Physics at RHIC - List of Participants

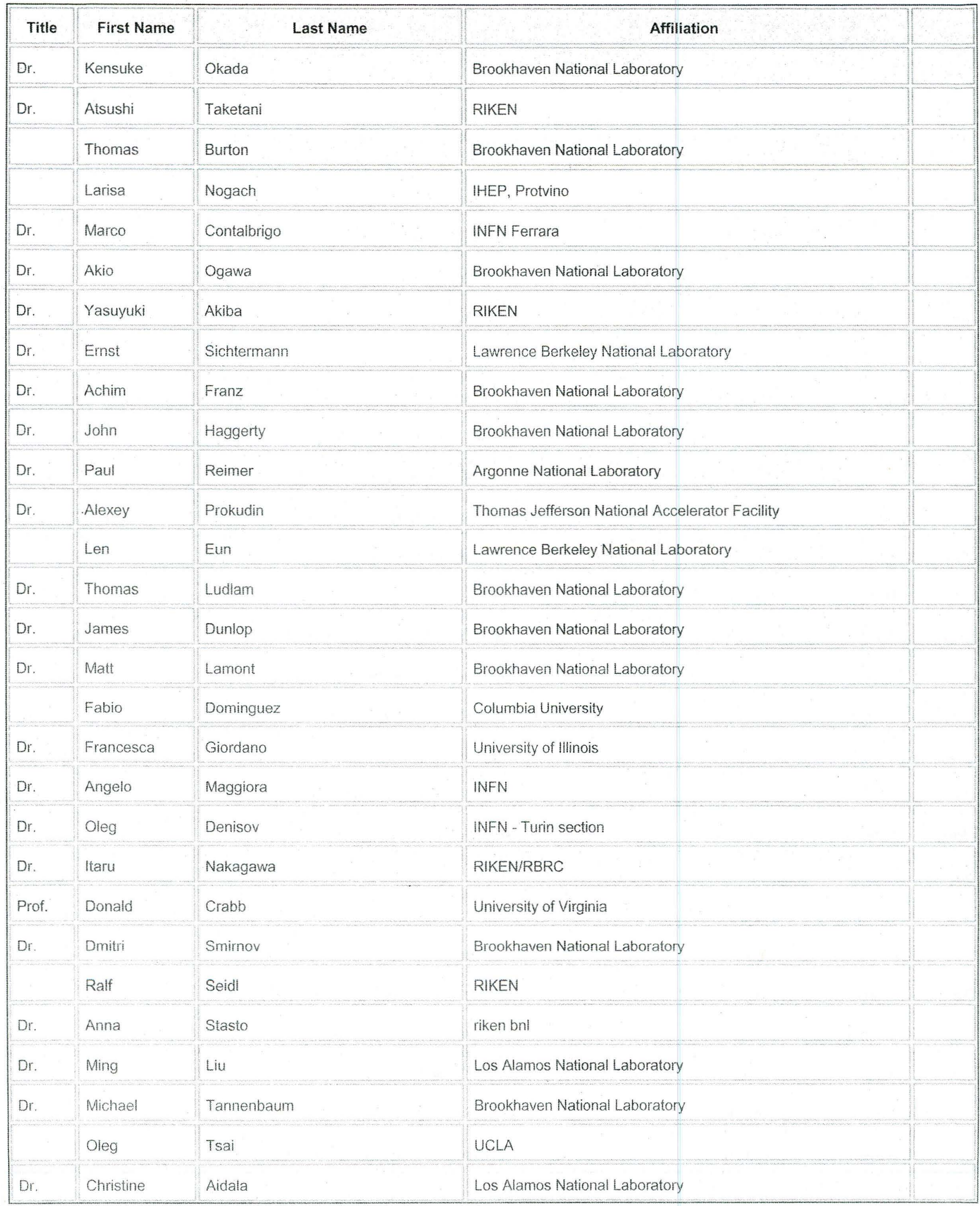




\begin{tabular}{|c|c|c|c|c|c|}
\hline Prof. & George & Sterman & & Stony Brook University & \\
\hline Prof. & Daniele & Panzien & & INFN & \\
\hline Prof. & Charles & Perdrisat & & William and Mary & \\
\hline Prof. & Vina & Punjabi & & Norfolk State University & \\
\hline Prof. & Jen-Chieh & Peng & & University of llinois & \\
\hline \multirow[t]{2}{*}{ Dr. } & Xiaodong & Jiang & & Los Alamos National Laboratory & \\
\hline & Jon & Engelage & & university of callfornia -ss & \\
\hline Prof. & Yuij & Koike & ) & Nigata University & \\
\hline \multirow[t]{3}{*}{ Prof. } & George & 190 & & UCLA & \\
\hline & Alfred & Mueller & & Columbia University & \\
\hline & Narbe & Kalantarians & & University of Virginia & \\
\hline Dr. & Ronald & Longacre & & Brookhaven National Laboratory & \\
\hline Dr. & Elke & Aschenauer & & Brookhaven National Laboratory & \\
\hline Dr. & William & Christie & & Brookhaven National Laboratory & \\
\hline Ms. & Xuan & $\mathrm{Li}$ & & Shandong University & \\
\hline \multirow[t]{2}{*}{ Prof. } & Huan & Huang & & UCLA & \\
\hline & Wolfram & Fischer & & Brookhaven National Laboratory & \\
\hline Dr. & Raju & Venugopalan & & Brookhaven National Laboratory & \\
\hline Prof. & Leonard & Gamberg & & Penn State University Berks & \\
\hline Dr. & Joseph & Seele & & MIT & \\
\hline Dr. & Marco & Radici & 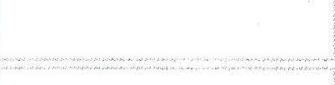 & INFN - Sezione di Pavia & \\
\hline Prof. & Kenneth & Barish & 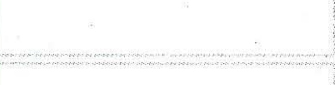 & UC Riverside & \\
\hline Dr. & Feng & Yuan & 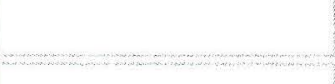 & RBRC & \\
\hline \multirow[t]{2}{*}{ Dr. } & Alessandro & Bacchetta & & University of Pavia and INFN Pavia & \\
\hline & Andreas & Metz & & Temple University & . \\
\hline Dr. & Bowen & Xiao & & Pennsylvania State University & \\
\hline Prof. & Mathias & Burkardt & & New Mexico State University & \\
\hline \multirow[t]{3}{*}{ Dr. } & Aram & Kotzinian & & Torino Uni\&INFN and YerPht & \\
\hline & Elena & Petreska & & Baruch, Graduate Center-CUNY & \\
\hline & Adrian & Dumitru & & Baruch/CUNY and RBRC & \\
\hline
\end{tabular}




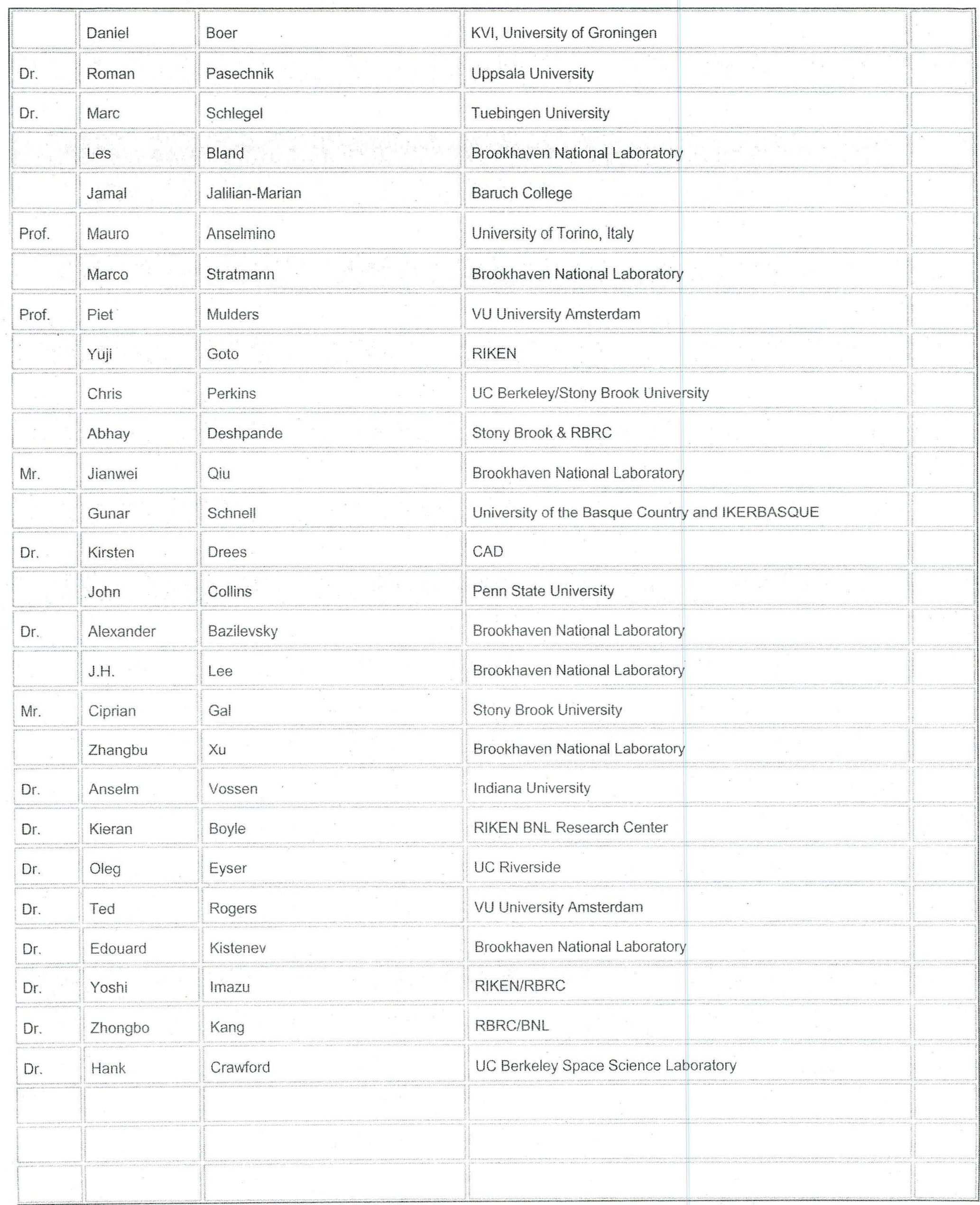




\section{RBRC Workshop Proceedings}

Volume 103 - Opportunities for Drell-Yan Physics at RHIC, May 11-13, 2011 - BNL-95236-2011

Volume 102 - Initial State Fluctuations and Final-State Particle Correlations, February 2-4, 2011 - BNL-94704-2011

Volume 101 - RBRC Scientific Review Committee Meeting, October 27-29, 2010 - BNL-94589-2011

Volume 100 - Summer Program on Nucleon Spin Physics at BNL, July 14-28, 2010

Volume 99 - The Physics of W and Z Bosons, BNL, June 24-25, 2010 - BNL-94287-2010

Volume 98 - Saturation, the Color Glass Condensate and the Glasma: What Have we Learned from RHIC? BNL, May 10-12, 2010 - BNL-94271-2010

Volume 97 - RBRC Scientific Review Committee Meeting, October 21-22, 2009 - BNL-90674-2009

Volume 96 - P-and CP-Odd Effects in Hot and Dense Matter, April 26-30, 2010 - BNL-94237-2010

Volume 95 - Progress in High-pT Physics at RHIC, March 17-19, 2010 - BNL-94214-2010

Volume 94 - Summer Program on Nucleon Spin Physics at LBL, June 1-12, 2009

Volume 93 - PHENIX Spinfest School 2009 at BNL - July 1 -31, 2009 - BNL-90343-2009

Volume 92 - PKU-RBRC Workshop on Transverse Spin Physics, June 30 - July 04, 2008 - BNL-81685-2008

Volume 91

RBRC Scientific Review Committee Meeting, Nov. 17 \& 18, 2008 - BNL-81556-2008

Volume 90 - PHENIX Spinfest School 2008 at BNL, August 4-8, 2008 - BNL-81478-2008

Volume 89 - Understanding QGP through Spectral Functions and Euclidean Correlators, April 23-25, 2008 - BNL-81318-

2008

Volume 88 - Hydrodynamics in Heavy Ion Collisions and QCD Equation of State, April 21-22, 2008 - BNL-81307-2008

Volume 87 - RBRC Scientific Review Committee Meeting - BNL-79570-2007

Volume 86 - Global Analysis of Polarized Parton Distributions in the RHIC Era, October 8, 2007 - BNL-79457-2007

Volume 85 - Parity Violating Spin Asymmetries at RHIC-BNL, April 26-27, 2007 - BNL - 79146-2007

Volume 84 - Domain Wall Fermions at Ten Years, March 15-17, 2007 - BNL-77857-2007

Volume 83 - QCD in Extreme Conditions, July 31-August 2, 2006 - BNL-76933-2006

Volume 82 - RHIC Physics in the Context of the Standard Model, June 18-23, 2006 - BNL-76863-2006

Volume 81 - Parton Orbital Angular Momentum (Joint RBRC/University of New Mexico Workshop), February 24-26, 2006 -

BNL-75937-

Volume 80 - Can We Discover the QCD Critical Point at RHIC?, March 9-10, 2006 - BNL 75692-2006

Volume 79 - Strangeness in Collisions, February 16-17, 2006 - BNL-79763-2008

Volume 78 - Heavy Flavor Productions and Hot/Dense Quark Matter, December 12-14, 2005 - BNL-76915-2006

Volume 77 - RBRC Scientific Review Committee Meeting, October 10-12, 2005 - BNL-52649-2005

Volume 76 - Odderon Searches at RHIC, September 27-29, 2005 - BNL-75092-2005

Volume 75 - Single Spin Asymmetries, June 1-3, 2005 - BNL-74717-2005

Volume 74 - RBRC QCDPC Computer Dedication and Symposium on RBRC QCDOC, May 26, 2005 - BNL-74813-2005

Volume 73

Volume 72 - RHIC Spin Collaboration Meetings XXXI (January 14, 2005), XXXII (February 10, 2005), XXXIII (March 11, 2005) - BNL-

Volume 71 Volume 70 volume 69 Volume 68 volume 67 volume 66 Volume 65 Volume 64 Volume 63 Volume 62 Volume 61 Volume 60 Volume 59 Volume 58 Volume 57 Volume 56 Volume 55 Volume 54 Volume 53 73866-2005

Classical and Quantum Aspects of the Color Glass Condensate - BNL-73793-2005

Stongly Coupled Plasmas: Electromagnetic, Nuclear \& Atomic - BNL-73867-2005

RBRC Scientific Review Committee Meeting - BNL-73546-2004

Workshop on the Physics Programme of the RBRC and UKQCD QCDOC Machines - BNL-73604-2004

High Performance Computing with BlueGene/L and QCDOC Architectures - BNL-

RHIC Spin Collaboration Meeting XXIX, October 8-9, 2004, Torino, Italy - BNL-73534-2004

RHIC Spin Collaboration Meetings XXVII (July 22, 2004), XXVIII (September 2, 2004) - BNL-73506-2004

Theory Summer Program on RHIC Physics - BNL-73263-2004

RHIC Spin Collaboration Meetings XXIV (05/21/04), XXV (05/27/04), XXVI (06/01/04) - BNL-72397-2004

New Discoveries at RHIC, May 14-15, 2004 - BNL-72391-2004

RIKEN-TODAI Mini Workshop on "Topics in Hadron Physics at RHIC", March 23-24, 2004 - BNL-72336-2004

Lattice QCD at Finite Temperature and Density - BNL-72083-2004

RHIC Spin Collaboration Meeting XXI, XXII, XXIII - BNL-72382-2004

RHIC Spin Collaboration Meeting XX - BNL-71900-2004

High pt Physics at RHIC, December 2-6, 2003 - BNL-72069-2004

RBRC Scientific Review Committee Meeting - BNL-71899-2003

Collective Flow and QGP Properties - BNL-71898-2003

RHIC Spin Collaboration Meetings XVII, XVIII, XIX - BNL-71751-2003

Theory Studies for Polarized pp Scattering - BNL-71747-2003 
Volume 52 - RIKEN School on QCD, "Topics on the Proton" - BNL-71694-2003

Volume 51 - RHIC Spin Collaboration Meetings XV, XVI - BNL-71539-2003

Volume 50 - High Performance Computing with QCDOC and BlueGene - BNL-71147-2003

Volume 49 - RBRC Scientific Review Committee Meeting - BNL-52679

Volume 48 - RHIC Spin Collaboration Meeting XIV - BNL-71300-2003

Volume 47 - RHIC Spin Collaboration Meetings XII, XIII - BNL-71118-2003

Volume 46 - Large-Scale Computations in Nuclear Physics using the QCDOC - BNL-52678

Volume 45 - Summer Program: Current and Future Directions at RHIC - BNL-71035

Volume 44 - RHIC Spin Collaboration Meetings VIII, IX, X, XI - BNL-71117-2003

Volume 43 - RIKEN Winter School - Quark-Gluon Structure of the Nucleon and QCD - BNL-52672

Volume 42 - Baryon Dynamics at RHIC - BNL-52669

Volume 41 - Hadron Structure from Lattice QCD - BNL-52674

Volume 40 - Theory Studies for RHIC-Spin - BNL-52662

Volume 39 - RHIC Spin Collaboration Meeting VII - BNL-52659

Volume 38 - RBRC Scientific Review Committee Meeting - BNL-52649

Volume 37 - RHIC Spin Collaboration Meeting VI (Part 2) - BNL-52660

Volume 36 - RHIC Spin Collaboration Meeting VI - BNL-52642

Volume 35 - RIKEN Winter School - Quarks, Hadrons and Nuclei - QCD Hard Processes and the Nucleon Spin - BNL-52643

Volume 34 - High Energy QCD: Beyond the Pomeron - BNL-52641

Volume 33 - Spin Physics at RHIC in Year-1 and Beyond - BNL-52635

Volume 32 - RHIC Spin Physics V - BNL-52628

Volume 31 - RHIC Spin Physics III \& IV Polarized Partons at High Q^2 Region - BNL 52617

Volume 30 - RBRC Scientific Review Committee Meeting - BNL-52603

Volume 29 - Future Transversity Measurements - BNL-52612

Volume 28 - Equilibrium \& Non-Equilibrium Aspects of Hot, Dense QCD - BNL-52613

Volume 27 - Predictions and Uncertainties for RHIC Spin Physics \& Event Generator for RHIC Spin Physics III - Towards Precision Spin Physics at RHIC - BNL-52596

Volume 26 - Circum-Pan-Pacific RIKEN Symposium on High Energy Spin Physics - BNL-52588

Volume 25 - RHIC Spin - BNL-52581

Volume 24 - Physics Society of Japan Biannual Meeting Symposium on QCD Physics at RIKEN BNL Research Center - BNL52578

Volume 23 - Coulomb and Pion-Asymmetry Polarimetry and Hadronic Spin Dependence at RHIC Energies - BNL-52589

Volume 22 - OSCAR II: Predictions for RHIC - BNL-52591

Volume 21 - RBRC Scientific Review Committee Meeting - BNL-52568

Volume 20 - Gauge-Invariant Variables in Gauge Theories - BNL-52590

Volume 19 - Numerical Algorithms at Non-Zero Chemical Potential - BNL-52573

Volume 18 - Event Generator for RHIC Spin Physics - BNL-52571

Volume 17 - Hard Parton Physics in High-Energy Nuclear Collisions - BNL-52574

Volume 16 - RIKEN Winter School - Structure of Hadrons - Introduction to QCD Hard Processes - BNL-52569

Volume 15 - QCD Phase Transitions - BNL-52561

Volume 14 - Quantum Fields In and Out of Equilibrium - BNL-52560

Volume 13 - Physics of the 1 Teraflop RIKEN-BNL-Columbia QCD Project First Anniversary Celebration - BNL-66299

Volume 12 - Quarkonium Production in Relativistic Nuclear Collisions - BNL-52559

Volume 11 - Event Generator for RHIC Spin Physics - BNL-66116

Volume 10 - Physics of Polarimetry at RHIC - BNL-65926

Volume 9 - High Density Matter in AGS, SPS and RHIC Collisions - BNL-65762

Volume 8 - Fermion Frontiers in Vector Lattice Gauge Theories - BNL-65634

Volume 7 - RHIC Spin Physics - BNL-65615

Volume 6 - Quarks and Gluons in the Nucleon - BNL-65234

Volume 5 - Color Superconductivity, Instantons and Parity (Non?)-Conservation at High Baryon Density - BNL-65105

Volume 4 - Inauguration Ceremony, September 22 and Non -Equilibrium Many Body Dynamics -BNL-64912

Volume 3 - Hadron Spin-Flip at RHIC Energies - BNL-64724

Volume 2 - Perturbative QCD as a Probe of Hadron Structure - BNL-64723

Volume 1 - Open Standards for Cascade Models for RHIC - BNL-64722 


\section{For information contact:}

Experimental Group Administrator

Ms. Susan Foster (PO)

RIKEN BNL Research Center

Building 510A; Room 2-70

Brookhaven National Laboratory

Upton, NY 11973-5000 USA

Phone: (631) 344-5864

Fax: (631) 344-2562

Email: sfoster@bnl.gov

Administrator to Nick Samios

Ms. Kerry Zendzian

RIKEN BNL Research Center

Bldg. 510A; Room 2-58

Brookhaven National Laboratory

Upton, NY 11973-5000 USA

Phone: (631) 344-3102

Fax: (631) 344-4906

Email: zendzian@bnl.gov
Theory Group Administrator

Ms. Pamela Esposito

RIKEN BNL Research Center

Bldg. 510A; Room 2-65

Brookhaven National Laboratory

Upton, NY 11973-5000 USA

Phone: (631) 344-3097

Fax: (631) 344-4067

Email: pesposit@bnl.gov

Homepage: http://www.bnl.gov/riken

RBRC RIKEN BNL Research Center workshops:

http://www.bnl.gov/riken/workshops.asp\#upcoming 


\section{RIKEN BNL RESEARCH CENTER \\ Opportunities for Drell-Yan Physics at RHIC}

Organizers: Elke Aschenauer (BNL), Les. Bland (BNL), Hank Crawford (U. of Berkeley), Yuji Goto (RIKEN/RBRC), Oleg Eyser (UC-Riverside), Zhongbo Kang (BNL), Anselm Vossen (U. of Indiana)

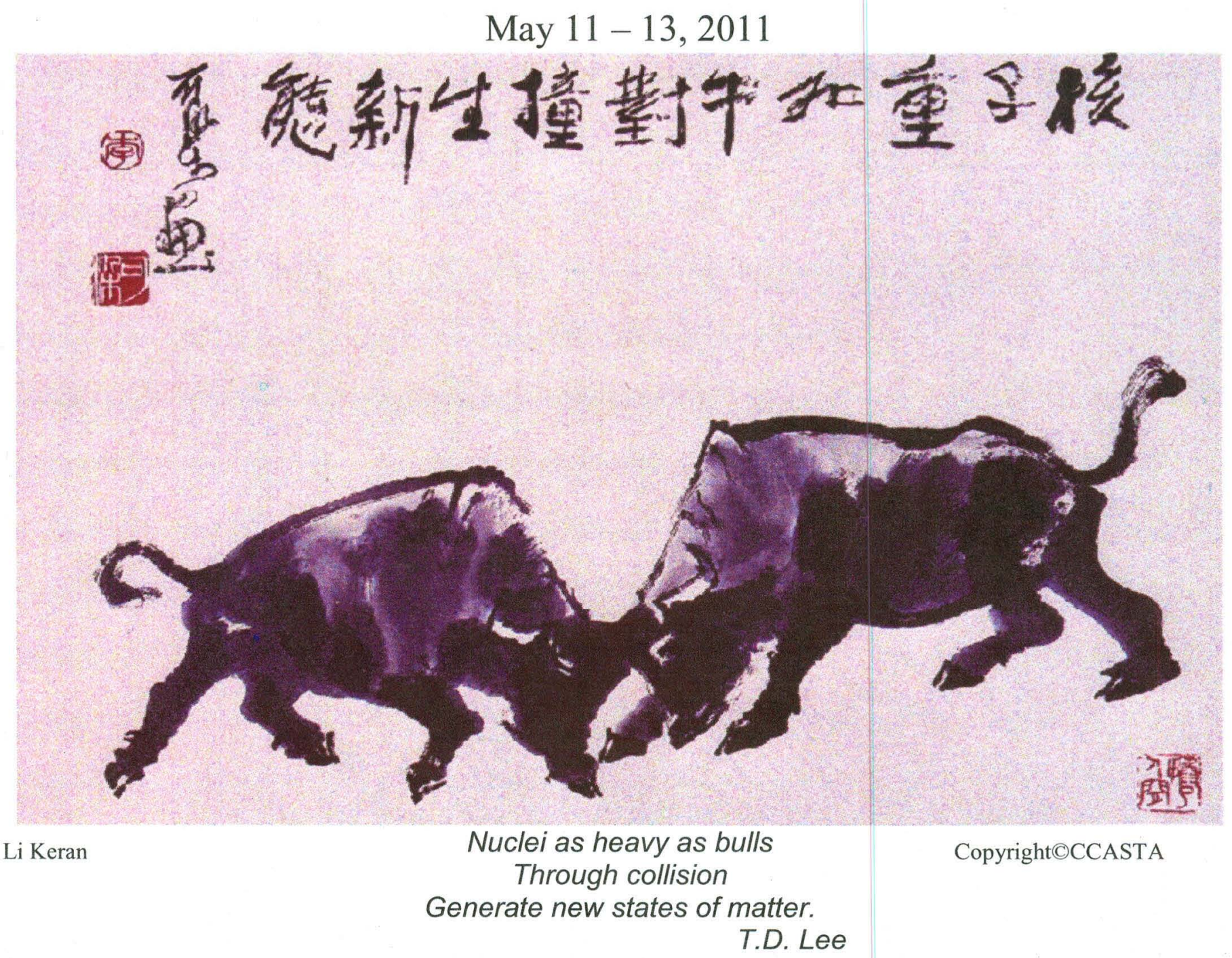

Speakers:

George Sterman

Jamal Jalian-Marian

Oleg Denisov

Wolfram Fischer

Roman Pasechnik

John Collins
Al Mueller

Feng Yuan

Jen-Chieh Peng

Jian-Wei Qiu

Alexey Prokudin

Gunar Schnell
Mauro Anselmino

Piet Mulders

Ernst Sichtermann

Andreas Metz

Alessandro Bacchetta

Zhongbo Kang
Daniel Boer

Ted Rogers

Ming Liu

Yuji Koike

Marc Schlegel

Bowen Xiao
Anna Stasto

Paul Reimer

Les Bland

Leonard Gambert

Raju Venugopalan

Aram Kotzinizn 